\title{
The two-photon decay of the $\frac{11}{2}^{-}$isomer of ${ }^{137} \mathrm{Ba}$ and mixed-symmetry states of ${ }^{92,94} \mathbf{Z r}$ and ${ }^{94} \mathbf{M o}$
}

\author{
Vom Fachbereich Physik \\ der Technischen Universität Darmstadt \\ zur Erlangung des Grades eines \\ Doktors der Naturwissenschaften (Dr. rer. nat.) \\ genehmigte \\ Dissertation \\ von \\ M.Sc. Christopher Walz \\ aus Wiesbaden
}

Referent: Prof. Dr. Dr. h.c. Norbert Pietralla

Korreferent: Dr. Heiko Scheit

Tag der Einreichung: 13. Juni 2014

Tag der Prüfung: 9. Juli 2014

Darmstadt 2014

D17 


\section{Abstract}

The first part of this thesis covers the two-photon decay of the $\frac{11}{2}^{-}$isomer in ${ }^{137} \mathrm{Ba}$. Clear evidence of this exotic decay mode is found, which opens up the possibility to use the twophoton decay probability as a general tool to investigate the structure of atomic nuclei. Details on the experiment are given, and the major challenges to successfully perform this difficult experiment are outlined. The two-photon decay experiment was conducted with the new $\mathrm{LaBr}_{3}$ detector ball which was setup from scratch in the framework of this thesis. The properties and performance of this powerful detector system - consisting of 18 large $\mathrm{LaBr}_{3}$-detectors - are discussed.

Furthermore, proton scattering at $200 \mathrm{MeV}$ has been performed on the nuclei ${ }^{92,94} \mathrm{Zr},{ }^{94,96} \mathrm{Mo}$ and ${ }^{70} \mathrm{Zn}$. The measured cross sections are compared to calculations in the framework of the Quasi-Particle Phonon Model (QPM). Conclusions about the validity of the QPM results are drawn. A comparison of the excitation cross sections of the $2_{1}^{+}$states and the one-phonon quadrupole states with mixed neutron and proton symmetry $\left(2_{\mathrm{ms}}^{+}\right)$allows to prove the special character of the $2_{\mathrm{ms}}^{+}$states independently of electromagnetic transition strengths. The possible existence of $3^{-}$and $4^{+}$states with mixed proton and neutron symmetry is discussed. Furthermore the origin of collectivity of low-lying states in spherical nuclei is investigated in the QPM.

\section{Zusammenfassung}

Der erste Teil dieser Dissertation behandelt den Zwei-Photonenzerfall des $\frac{11}{2}^{-}$Isomers in ${ }^{137} \mathrm{Ba}$. Ein Nachweis dieser exotische Zerfallsmode ist gelungen. Dies ermöglicht es, den ZweiPhotonenzerfall als neue Methode zur Untersuchung spezieller Akspekte der Struktur von Atomkernen zu verwenden. Das Experiment ist im Detail beschrieben und die Hauptschwierigkeit für eine erfolgreiche Durchführung eines Zwei-Photonenzerfalls Experiments werden diskutiert. Im Rahmen dieser Arbeit wurde ein neuer $\mathrm{LaBr}_{3}$-Detektorball aufgebaut. Die Eigenschaften und die Performance dieses Detektorsystems - bestehend aus 18 großen $\mathrm{LaBr}_{3}$-Detektoren - werden präsentiert.

Die Kerne ${ }^{92,94} \mathrm{Zr}$, ${ }^{94,96} \mathrm{Mo}$ und ${ }^{70} \mathrm{Zn}$ wurden in einem Protonenstreuexperiment bei einer Energie von $200 \mathrm{MeV}$ untersucht. Die gemessenen Anregungs-Wirkungsquerschnitte wurden mit Modellrechnungen im Rahmen des Quasi-Particle Phonon Model (QPM) verglichen und Rückschlüsse über die Gültigkeit des QPMs konnten gezogen werden. Ein Vergleich der Wirkungsquerschnitte der $2_{1}^{+}$Zustände mit denen von Zuständen mit gemischter Neutronenund Protonensymmetrie $\left(2_{\mathrm{ms}}^{+}\right)$, erlauben den gemischt-symmetrischen Charakter der $2_{\mathrm{ms}}^{+}$ Zustände zu beweisen, unabhängig von elektromagnetischen Übergangsstärken. Des Weiteren wird die mögliche Existenz von $3^{-}$und $4^{+}$Zuständen mit gemischter Protonen- und Neutronensymmetrie diskutiert. Zusätzlich bietet das QPM Einblicke in die Entstehung von niederenergetischen, kollektiven Zuständen in sphärischen Kernen. 


\section{Contents}

$\begin{array}{lr}\text { 1. Introduction } & 1\end{array}$

2. Theoretical background of the two-photon decay experiment 9

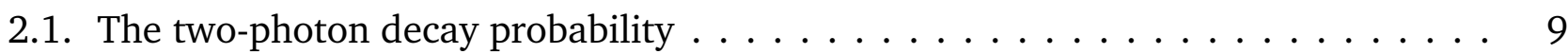

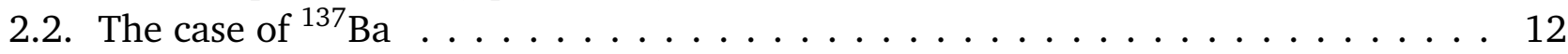

3. Experimental setup of the two-photon decay experiment 17

3.1. Lead shield and choice of detector arrangement . . . . . . . . . . . 17

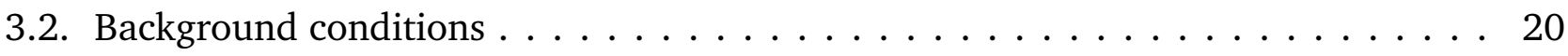

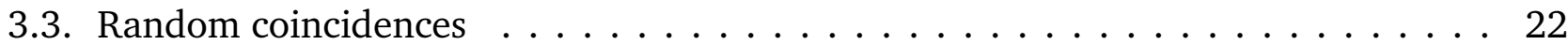

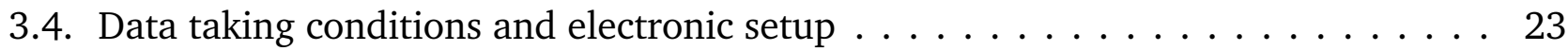

4. Data analysis and results of the two-photon decay experiment 27

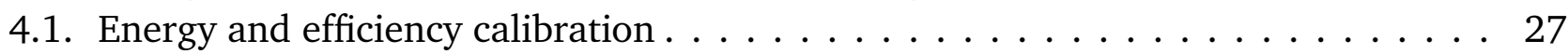

4.2. Subtraction of the time random coincidences $\ldots \ldots \ldots \ldots$

4.3. Background conditions of the two-photon decay experiment $\ldots \ldots \ldots$. . . . . 30

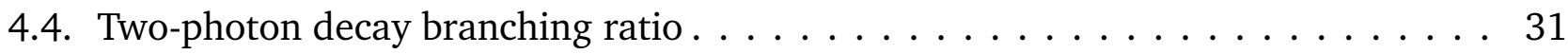

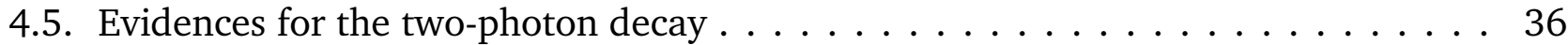

4.6. The energy sharing distribution $\ldots \ldots \ldots \ldots \ldots$

5. Theoretical background of the proton scattering experiments 41

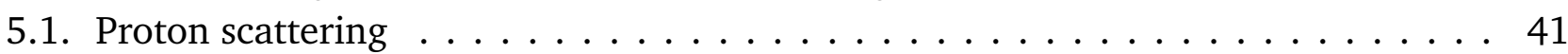

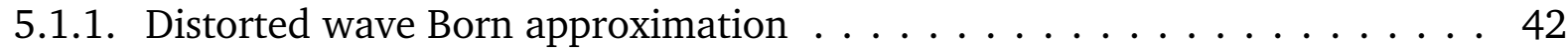

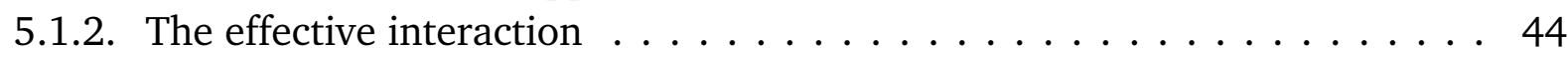

5.1.3. Microscopic optical model . . . . . . . . . . . . . . . . . . . . 44

5.2. Mixed-symmetry states in the Interacting Boson Model $2 \ldots$. . . . . . . . . . . 45

5.3. The Quasiparticle Phonon Model . . . . . . . . . . . . . . . . . . . . . 49

5.3.1. Outline of the model . . . . . . . . . . . . . . . . . . 49

5.3.2. Electromagnetic transition strengths and transition densities . . . . . . . . 54

5.3.3. Definition of a mixed-symmetry state in the framework of the QPM $\ldots . .55$

6. The experimental setup at iThemba LABS 57

6.1. The iThemba Laboratory . . . . . . . . . . . . . . . . . . . . . 57

6.2. The K600 magnetic spectrometer . . . . . . . . . . . . . . . . . . 57

6.3. The focal plane detector package $\ldots \ldots \ldots \ldots \ldots$

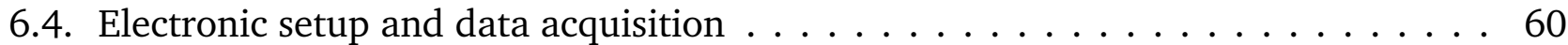

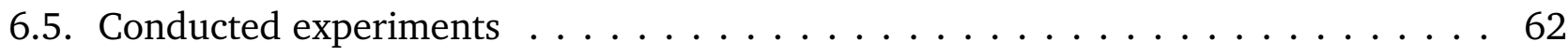


7. Data analysis and results of the proton scattering experiments

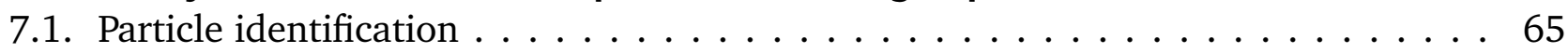

7.2. Determination of the focal plane coordinates $\ldots \ldots \ldots 6$

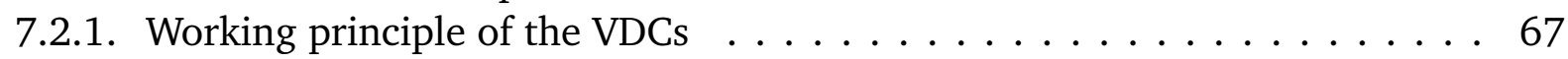

7.2.2. Conversion of drift times to drift distances . . . . . . . . . . . 68

7.2.3. The lookup table shift . . . . . . . . . . . . . . . . . . . . . . . . . . . . . . . . .

7.3. Calibration of the scattering angle $\ldots \ldots \ldots \ldots \ldots$

7.4. Correction of higher-order aberrations and kinematic effects . . . . . . . . 71

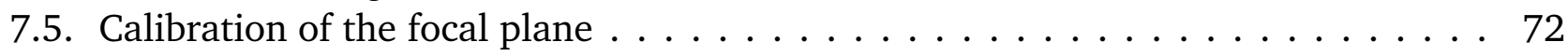

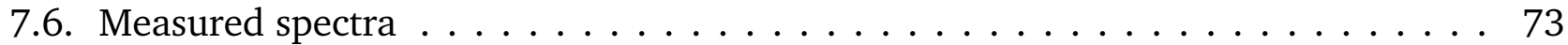

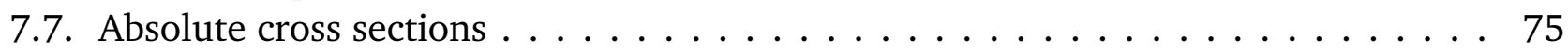

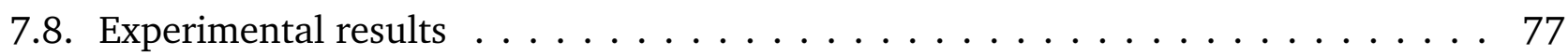

7.8.1. Results of the ${ }^{92} \operatorname{Zr}\left(p, p^{\prime}\right)$ experiment . . . . . . . . . . . . . . 77

7.8.2. Results of the ${ }^{94} \operatorname{Zr}\left(p, p^{\prime}\right)$ experiment . . . . . . . . . . . . . . 80

7.8.3. Results of the ${ }^{94} \mathrm{Mo}\left(p, p^{\prime}\right)$ experiment $\ldots \ldots \ldots \ldots \ldots$

7.8.4. Results of the ${ }^{96} \mathrm{Mo}\left(p, p^{\prime}\right)$ experiment $\ldots \ldots \ldots \ldots \ldots$

7.8.5. Results of the ${ }^{70} \mathrm{Zn}\left(p, p^{\prime}\right)$ experiment $\ldots \ldots \ldots \ldots$. . . . . . . 89

8. Interpretation $\quad 95$

8.1. Comparison of QPM calculations to experiment $\ldots \ldots \ldots \ldots 6$

8.1.1. Ground state properties . . . . . . . . . . . . . . . . . 96

8.1.2. Quadrupole states . . . . . . . . . . . . . . . . . . . . . . . . . . . . . . . . . . .

8.1.3. Hexadecapole states . . . . . . . . . . . . . . . . . . . . 103

8.1.4. Octupole and $5^{-}$states . . . . . . . . . . . . . . . . 108

8.1.5. Understanding the structure of low-lying natural parity states of ${ }^{92,94} \mathrm{Zr}$

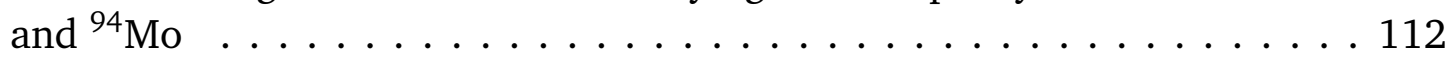

8.2. Proton and neutron transition matrix elements to the ground state:

Sensitivity to mixed-symmetry states and origin of collectivity $\ldots \ldots \ldots$. . . . 116

8.3. New signature of one-phonon quadrupole mixed-symmetry states . . . . . . . . 122

$\begin{array}{lr}\text { 9. Summary \& outlook } & 129\end{array}$ 
B. Results of the proton scattering experiments 135

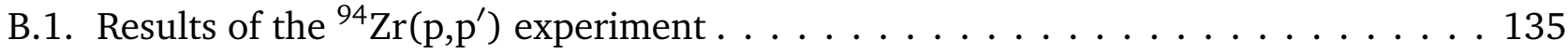

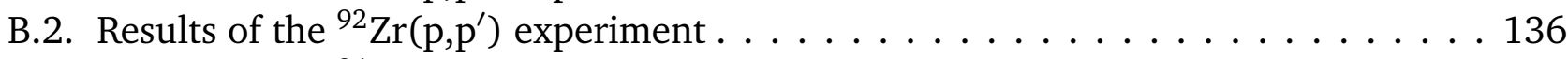

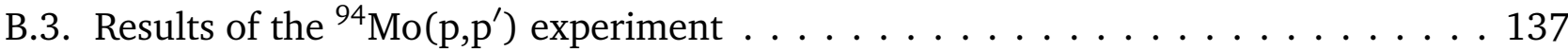

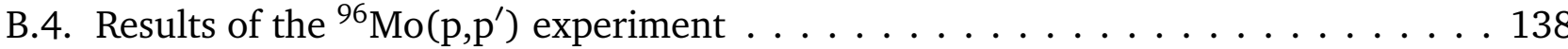

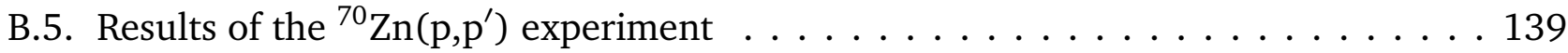

$\begin{array}{ll}\text { Bibliography } & 139\end{array}$

$\begin{array}{ll}\text { List of Figures } & 148\end{array}$

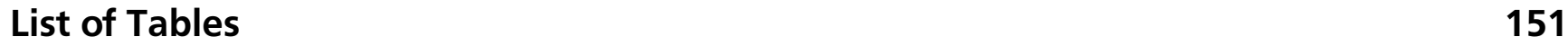

$\begin{array}{ll}\text { Danksagung } & 154\end{array}$

$\begin{array}{ll}\text { Lebenslauf } & 156\end{array}$ 


\section{Introduction}

The atomic nucleus consists of protons and neutrons which interact via the strong, electromagnetic and weak forces. It is a complex, finite many-body system of up to $\sim 300$ constituents. Atomic nuclei are the cores of atoms and are responsible for essentially all visible matter in the universe. The aim of nuclear structure physics is to achieve a comprehensive description of all nuclei based on the underlying-theory of Quantum Chromodynamics (QCD).

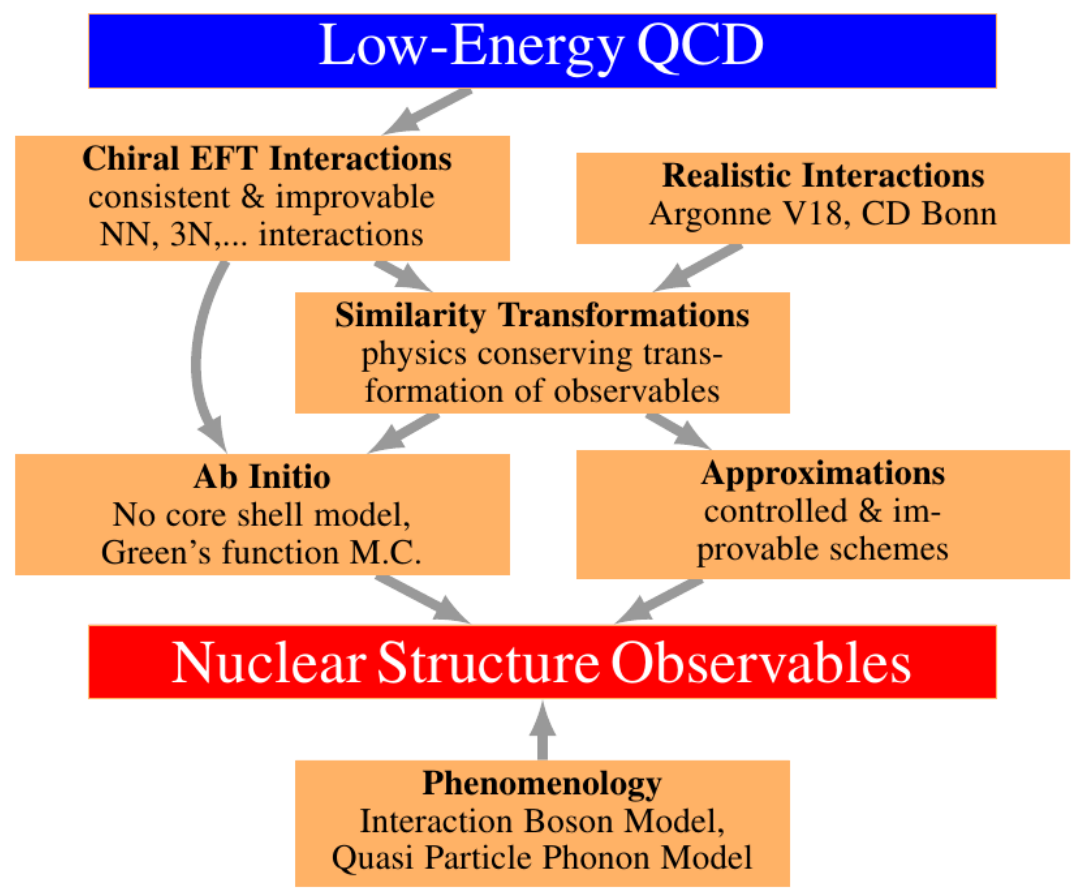

Figure 1.1: Theoretical approaches to tackle the nuclear many-body problem. For details see text. (taken, and slightly changed, from Refs. [Rot11, Wal10])

Despite significant efforts in recent decades, nuclei are not fully understood yet and many open theoretical problems remain. The theoretical strategy to tackle the nuclear many-body problem is displayed in Fig. 1.1. The problem can be decomposed in two major challenges. First of all the interaction between nucleons - the nuclear force - is not a fundamental one. It can be considered as a residual color force which is analogous to the van der Waals force between neutral molecules [Epe09]. In order to put nuclear structure calculations on firm grounds it is necessary to derive the effective nuclear force from the fundamental theory of quarks and gluons: QCD. In recent years advances were made in this field. Today nuclear potentials are available from chiral effective field theory [Mac11] which are based on the symmetries of QCD and are able to describe the nucleon-nucleon phase shifts. Furthermore these potentials allow a systematic inclusion of the small but necessary three nucleon forces and it is possible to assign a theoretical error to calculated observables [Epe09]. More phenomenological nuclear potentials like Argonne V18 [Wir95] and CD Bonn [Mac01], which were extensively used in the past, do not exhibit these advantages. 
A large variety of theoretical methods are available to solve the many-body problem with the nuclear potential of choice. In principle one would like to solve the problem ab initio i.e. without further approximations. Such methods, like the no core shell model [Nav00] or the Green's function Monte-Carlo approach [Pie05], are available and achieve a satisfactory description of the properties of light nuclei. Their application, however, is limited to nuclei with $\mathrm{A} \lesssim 12$ due to dramatically increasing dimensionality of the problem with increasing nucleon number. In order to describe heavy nuclei one has to rely on controlled approximations. Well known examples are the Hartree-Fock approach, random-phase approximations, coupled cluster methods [Kow04] or the no core shell model with an importance truncation [Rot07]. Each many-body methods uses a truncated Hilbert space and it is not suitable to use the bare nucleon-nucleon potential because of the strong correlations (i.e. the mixing of basis states) which are induced. With a so called similarity transformation which adapts the bare potential to the available model space, one can tackle this problem and improve the convergence behavior. Examples for widely used similarity transformation are the Similarity Renormalization Group [Bog03] and the Unitary Correlation Operator Method [Fel98, Nef03].

Today there are still many purely phenomenological nuclear structure models in use, as for example the Interacting Boson Model (IBM) [Ari75, Ari78] or the Quasi-Particle Phonon Model (QPM) [Ber99]. These models are still useful to design experiments and to obtain an understanding of a particular nuclear structure phenomenon.

Other fields of nuclear physics would strongly benefit from a reliable nuclear structure theory which is able to describe nuclei of all masses. One of the main aims of nuclear astrophysics is the modeling of the synthesis of heavy elements in explosive astrophysical scenarios [Nup10]. Approximately half of the elements heavier than iron are attributed to the so called rapid neutron capture process (r-process) [Bur57]. The r-process occurs in extremely neutron rich nuclei. Some of those nuclei will not be accessible in experiment even with the next generation of radioactive beam facilities. Particularly for this case, a model is necessary, which can be reliably extrapolate to neutron rich nuclei in order to provide firm nuclear structure input for determining the r-process path and ultimately understanding the element abundances in our universe. In general the theoretical tools developed to describe the nuclear many-body system can also be applied to improve the description of other complex many-body systems, e.g. Bose-Einstein condensates [Jin96].

A theoretical approach can only be validated by a comparison to experiment. Therefore the aim of each experimental work is ultimately to provide new or more accurate observables. In order to conduct a stringent test of a nuclear structure model it is important to do a comparison to many different observables which are sensitive to different aspects of the nuclear wave functions. For example a model which is able to accurately describe the energies of the low-lying states in a given nucleus can be inaccurate on the transition strengths.

In the last two decades high intensity and high energy radioactive beams have been developed at facilities like NSCL [NSC], GSI [GSI], RIKEN [Rik] and REX-ISOLDE [ISO]. These allow for the study of many more of the $6000-8000$ nuclei than only the $\sim 300$ stable nuclei. Pushing the nuclear many-body system to extreme conditions regarding e.g. the proton-to-neutron-ratio, binding energy or angular momentum [Nup04] has proven to be a successful way to obtain new insights in the properties of the nuclear force. Many interesting phenomena were discovered, for example neutron skins, halo nuclei [Tan85] and exotic shapes [Gaf13]. Additionally some of the 'basic truths' which were thought to be valid for decades, were revisited. The most striking examples are the change of the magic numbers and shell evolution, the size of nuclei do not 
scale with $A^{1 / 3}$ and the neutron drip line is much further away from the valley of stability then originally anticipated [Nup04]. Describing these new features and observables is a challenging task for modern nuclear structure models. In the next years new radioactive beam facilities will go into service: FAIR [FAI], FRIB [FRI], SPIRAL2 [SPI] and others which will significantly improve our understanding of nuclei.

Although the focus of nuclear structure physics has shifted in recent years to radioactive beam physics, the research on stable nuclei is far from being complete. The experimental work done in the framework of this thesis covers three separate topics which contribute to the understanding of stable nuclei.

An important observable in nuclear structure physics are the diagonal electric dipole polarizability [Rei10, Kra87] and the diagonal magnetic susceptibility [Kra87] which are proportional to the sum over all $B(E 1)$-values ( $B(M 1)$-values) weighted with the inverse excitation energy of the corresponding state. In order to determine these quantities a measurement of the electric and magnetic dipole responses over the full energy range is necessary. This is experimentally very challenging and so far proton scattering at zero degrees [Tam09] is the only method which allows to measure the full dipole responses below and above the particle threshold in one experiment. However even this advanced method exhibit inconsistencies with $\left(\gamma, \gamma^{\prime}\right)$-data which are not resolved yet [Mar14]. These observables can be used e.g. to restrict the parameters of the equation of state [Tew13] and to determine the spin quenching factors [Kra87], i.e. they directly address fundamental open problems in nuclear structure physics and astrophysics. Therefore it is highly desirable to find alternative observables to the diagonal electric dipole polarizability and diagonal magnetic susceptibility which can be used for the same investigations but are more reliable and easier to measure.
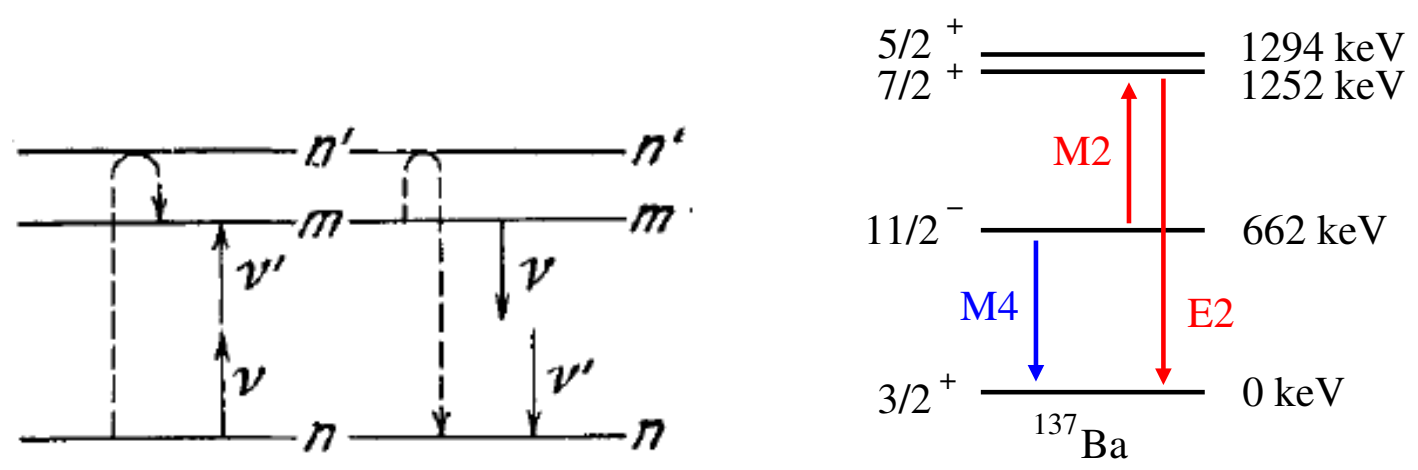

Figure 1.2: The two-photon absorption and emission processes through the intermediate virtual state $n^{\prime}$ (taken from Ref. [Goe30]). The dashed lines denote the behavior of the atom (or nucleus) and the solid arrows show the two absorbed or emitted photons with frequencies $v$ and $v^{\prime}$ (left-hand side). On the right-hand side the $\frac{11}{2}^{-}$state of ${ }^{137} \mathrm{Ba}$ decays via a $M 4$-transition and the emission of a single photon to the ground state (blue arrow). One possible path of the two-photon decay through the $\frac{7}{2}^{+}$state is also shown (red arrows).

In 1930 Maria Göppert-Mayer investigated for the first time the two-photon decay in atomic physics in her doctoral thesis [Goe30]. In this rather exotic electromagnetic process - which is a second order process - an excited state decays over an intermediate virtual state to a lowerlying state and emits two photons in coincidence. Their sum energy equals the transition energy 
while the energy sharing follows a broad energy distribution which depends on the multipole characters. On the left hand side of Fig. 1.2 - which is taken from Maria Göppert-Mayer's thesis [Goe30] - this process is shown schematically together with the opposite effect i.e. the absorption of two photons. Interestingly the two-photon decay probability involves a sum over all states $\left(n^{\prime}\right)$ of the atom which can be connected by the appropriate transition operators to the initial $(n)$ and final states $(m)$, i.e. it is an integral quantity. This makes the two-photon decay probability to a unique observable which is sensitive to the whole structure of the investigated many-body system and allows to obtain insights beyond the properties of single states like energy and transition strengths. In atomic physics this quantity was studied extensively from the theoretical [Mok04] as well as from the experimental side [Ila06] and has indeed proven to be a valuable observable.

In nuclear physics one can expect a similar decay mode between two states of the nucleus [Kra87]. However the experimental information is in contrast to atomic physics rather sparse. So far the two-photon decay probability has been measured for the $0_{2}^{+} \rightarrow 0_{1}^{+}$transitions in ${ }^{16} \mathrm{O}$ [Kra87], ${ }^{90} \mathrm{Zr}$ [Sch84, Kra87] and ${ }^{40} \mathrm{Ca}$ [Sch84, Kra87] only. This unsatisfactory situation is due to experimental problems to measure the two-photon decay if the one photon decay is allowed [Mus88]. For a $0_{i}^{+} \rightarrow 0_{f}^{+}$transition the one-photon decay is forbidden by angular momentum conservation. In addition the nuclei ${ }^{16} \mathrm{O},{ }^{90} \mathrm{Zr}$ and ${ }^{40} \mathrm{Ca}$ have in common that the $0_{2}^{+}$ state is also the first excited state. One can excite this state selectively and therefore it is not necessary to deal with the one-photon decays of other excited states. For almost all even-even nuclei, however, the first excited state is a $2^{+}$state. Clearly, if the two-photon decay probability should become a general tool to investigate systematically nuclear structure one has to find experimentally a way to measure the two-photon decay if the one-photon decay is present.

In Ref. [Kra87] it was shown in a schematic model that the two-photon decay probability is indeed closely related to the diagonal magnetic dipole suceptibility. Therefore one can hope that the two-photon decay probability is a useful observable to address some of the problems which are usually considered using the diagonal electric dipole polarizibility and diagonal magnetic dipole suceptibility. However whether this is really possible, has to be answered by theory with a realistic nuclear structure model.

The aim of the first part of this thesis is to overcome the 'experimental hurdle' which prevents the two-photon decay to become a general tool for nuclear structure research i.e. it is tried to measure the two-photon decay if the one-photon decay is allowed. It was decided to start with a radioactive source - namely ${ }^{137} \mathrm{Cs}$ - in order to conduct the experiment in a controlled environment i.e. without beam related background. ${ }^{137} \mathrm{Cs}$ decays via a $\beta^{-}$-decay to the $\frac{11^{-}}{2}$ state of ${ }^{137} \mathrm{Ba}$. As shown in Fig. 1.2 this state decays to the ground state via a M4-transition emitting a single photon. The two-photon decay proceeds via higher-lying intermediate states. One possible paths via the $\frac{7}{2}^{+}$state is displayed in Fig. 1.2.

The measurement of the two-photon decay was previously attempted in literature for the case of a $2_{1}^{+} \rightarrow 0_{1}^{+}$transition [Mus88]. This attempt failed which was in parts due to the usage of $\mathrm{NaI}$ detectors which have a limited energy and time resolution. In the recent years the new scintillator material $\mathrm{LaBr}_{3}$ was developed [Leo01]. This type of material has superior properties compared to conventional scintillators like $\mathrm{NaI}$ and $\mathrm{BaF}_{2}$ in terms of energy resolution, time resolution and efficiency [Sai]. The availability of $18 \mathrm{LaBr}_{3}$ crystals at the institute of nuclear physics in Darmstadt, which were bought in 2011 and 2012, was one of the motivations to attempt a new measurement of the two-photon decay probability with a better setup than in the 
past. Beforehand it is necessary to setup up the $\mathrm{LaBr}_{3}$-detector ball. This was also done in the framework of this thesis. This comprises the design of the detector shell and a support structure, the setup of a digital data acquisition system, design of the firmware for the used digitizer, choice of a suitable photomultiplier and voltage divider, extensive testing of all components and other things. The usage of this new powerful detector ball is not limited to the investigation of the two-photon decay. Possible further applications are $\left(e, e^{\prime} \gamma\right)$-coincidence measurements [Pap85] with the QCLAM spectrometer [Kni91, Lue95] and $\left(\gamma, \gamma^{\prime} \gamma^{\prime}\right)$-experiments with the photon tagger NEPTUN [Sav10] at the electron accelerator S-DALINAC in Darmstadt. Due to compactness of the $\mathrm{LaBr}_{3}$-detectors and the robust detector shell they are easily transportable and can be used at other facilities too. The setup of the $\mathrm{LaBr}_{3}$-ball is not discussed explicitly in this thesis. Some details on its properties and performance are given in Chapters 3 and 4.

The proton-neutron residual interaction is known to be responsible for configuration mixing [Gol53] and hence for many fundamental nuclear structure phenomena like the formation of low-lying collective excitations or the shape phase transition from spherical to deformed nuclei [Iac00]. Clearly, a probe which gives information on the strength of this residual interaction is of high value to understand the nuclear force. In 1984 the first example of a so called quadrupole mixed-symmetry state [Pie08] was discovered in spherical nuclei by Hamilton et al. [Ham84]. This excitation mode has the property to be anti-symmetry with respect to the proton-neutron degree of freedom [Hey86]. Its formation mechanism can be understood in a simple two-state model [Hey86] which can be justified by more advanced nuclear structure models (see Sect. 8.1.2). Starting from two unperturbed states in the proton and neutron systems $\left|2_{\pi}^{+}\right\rangle$and $\left|2_{v}^{+}\right\rangle$, one obtains two states - after considering the proton-neutron interaction with wave functions

$$
\begin{aligned}
& \left|2_{1}^{+}\right\rangle=\alpha\left|2_{\pi}^{+}\right\rangle+\beta\left|2_{v}^{+}\right\rangle \\
& \left|2_{\mathrm{ms}}^{+}\right\rangle=\beta\left|2_{\pi}^{+}\right\rangle-\alpha\left|2_{v}^{+}\right\rangle .
\end{aligned}
$$

The $2_{1}^{+}$state is the first excited $2^{+}$state in spherical nuclei and the $2_{\mathrm{ms}}^{+}$state is the mixedsymmetry state. The properties of both states depend on the degree of mixing (determined by the amplitudes $\alpha$ and $\beta$ ) i.e. on the strength of the proton-neutron residual interaction and the energy difference of the unperturbed states. Hence a systematic investigation of the properties of mixed-symmetry states clearly helps to understand the proton-neutron residual interaction and the underlying shell structure.

The main experimental signature of a quadrupole mixed-symmetry state is a large $B(M 1)$ value of the order of $\sim 1 \mu_{N}^{2}$ to the $2_{1}^{+}$state [Pie08]. Today a large number of quadrupole mixedsymmetry states have been observed in nuclei based on this signature as shown in Fig. 1.3 (yellow squares). In order to fully understand a nuclear excitation mode it is important to investigate its properties with different probes which are sensitive to different parts of the wave function. The aim of this thesis is to investigate the quadrupole mixed-symmetry states in ${ }^{92,94} \mathrm{Zr}$, ${ }^{94,96} \mathrm{Mo}$ and ${ }^{70} \mathrm{Zn}$ with inelastic proton scattering. This reaction is in contrast to $B(E 2)$-values also sensitive to the neutron part of the wave functions and gives information on the radial structure of the excitation. In this thesis it is shown that transition radii are sensitive to the special character of a mixed-symmetry state. They can be used to test model predictions for mixedsymmetry states and allow to prove the mixed-symmetric character of states independently of absolute $B(M 1)$-values (with an appropriate filling of the valence orbitals). Furthermore the possible existence of $3^{-}$and $4^{+}$one-phonon mixed-symmetry states is discussed. 


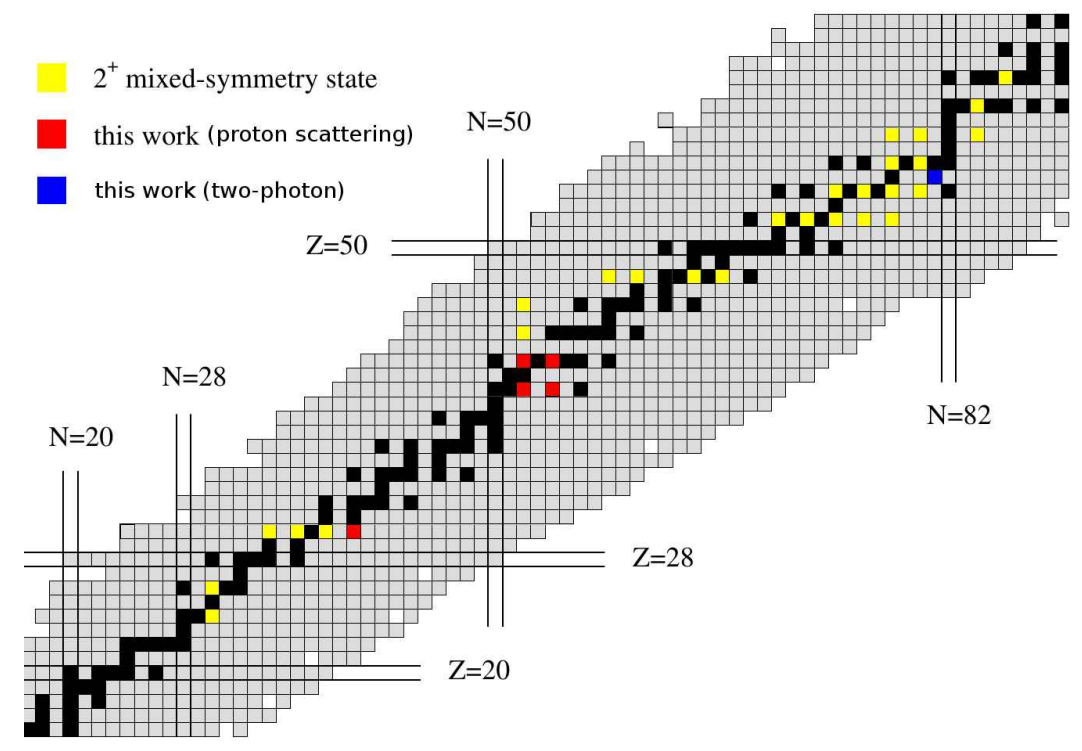

Figure 1.3: Known one-phonon quadrupole mixed-symmetry states (yellow squares). The nuclei ${ }^{92,94} \mathrm{Zr},{ }^{94,96} \mathrm{Mo}$ and ${ }^{70} \mathrm{Zn}$ investigated in the proton scattering experiments are in red. The two-photon decay experiment is performed with the nucleus ${ }^{137} \mathrm{Ba}$ which is in blue (taken, and slightly changed, from Ref. [Wal10]).

The outline of this thesis is as follows. In Chapter 2 the theory of the two-photon decay is presented with an emphasis on the situation in the investigated nucleus ${ }^{137} \mathrm{Ba}$ and guidelines for experiment following from theoretical considerations are given. The next chapter discusses the experimental setup to measure the two-photon decay and defines the key parameters for a successful experiment.The data analysis and results of the two-photon decay experiment are summarized in Chapter 4.

The second part of this thesis is about proton scattering experiments on the nuclei ${ }^{92,94} \mathrm{Zr}$, ${ }^{94,96} \mathrm{Mo}$ and ${ }^{70} \mathrm{Zn}$. The theoretical background of proton scattering and the nuclear structure model used to interpret the experimental data are outlined in Chapter 5 . The experiments were performed at the iThemba facility [iTH] and details on the experimental setup [Nev11] and the experiment itself are given in Chapter 6. Next the different steps of the data analysis are discussed i.e. how to obtain from the raw data of the wire chambers the final angular distributions of the cross sections. In Chapters 8 the results are discussed and a theoretical analysis is done in the framework of the QPM. The QPM calculations are compared to the proton scattering cross sections and conclusions are drawn about the validity of the QPM results. The possible existence of hexadecapole and octupole mixed-symmetry states is also discussed in this chapter. In Sect. 8.2 the origin of collectivity of low-lying states are investigated in the framework of the QPM. Finally in Sect. 8.3 a new signature is introduced to prove the mixedsymmetric character of the corresponding $2^{+}$states in ${ }^{92,94} \mathrm{Zr}$ and ${ }^{94} \mathrm{Mo}$. Chapter 9 summarizes the work done in this thesis and gives a roadmap how to proceed with the research of the two-photon decay.

Some topics of this thesis were already discussed in the author's master thesis [Wal10]. To be specific the analysis and results of the ${ }^{92} \mathrm{Zr}$ and ${ }^{94} \mathrm{Mo}$ proton scattering data were also presented in Ref. [Wal10]. However the data was reanalyzed in this doctoral thesis which indeed resulted in additional insights like the angular distribution of the $4_{2}^{+}$state in ${ }^{94} \mathrm{Mo}$. Furthermore the new signature for a quadrupole mixed-symmetry state and the properties of hexadecapole and 
octupole mixed-symmetry were also discussed in the master thesis. However the results were significantly extended and completed in this doctoral thesis. Parts of this thesis were already published in Ref. [Wal11]. 


\section{Theoretical background of the two-photon decay experiment}

\subsection{The two-photon decay probability}

The theory of the two-photon decay has been studied many times in literature [Gre62, Gre63, Gre65]. A complete description is given by Friar in Ref. [Fri75]. Based upon his results the authors of Ref. [Kra87] further extended the theoretical analysis with an emphasis on $0_{2}^{+} \rightarrow 0_{1}^{+}$ transitions. The crucial differential two-photon decay probability is only given for the $0_{2}^{+} \rightarrow 0_{1}^{+}$ case in Ref. [Kra87]. J. Millener derived this quantity for the general case which is needed for the investigation of ${ }^{137} \mathrm{Ba}$ [Mil13a, Mil13b].

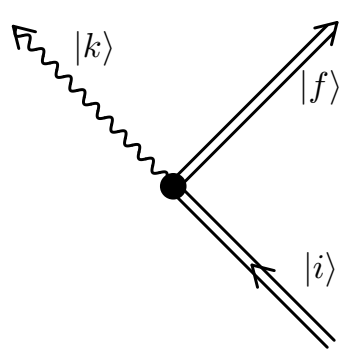

(a)

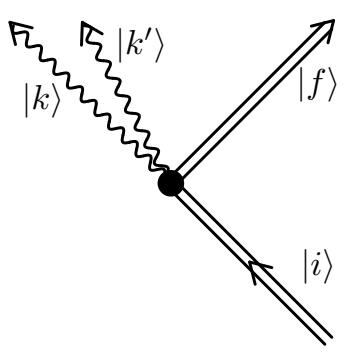

(b)

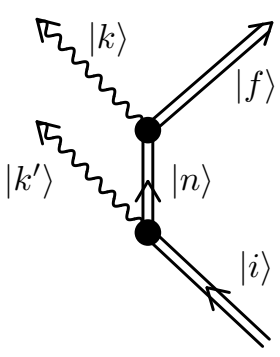

(c)

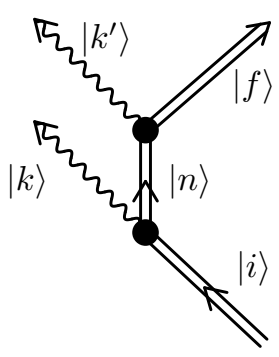

(d)

Figure 2.1: Feynman diagrams of the amplitudes contributing to the one-photon (a) and twophoton decay $(b-d)$ : The second order resonance amplitudes $(c+d)$ and the first order 'seagull' amplitude (b). Taken, and slightly changed, from Ref. [Fri75].

The interaction between the nucleus and an electromagnetic radiation field can be written as (Gaussian units with $\hbar=c=1$ and $\alpha=e^{2}$ ) [Fri75]

$$
H_{\text {int }}=\int j_{\mu}(x) A^{\mu}(x) d^{3} x+\frac{1}{2} \int B_{\mu v}(x, y) A^{\mu}(x) A^{v}(y) d^{3} x d^{3} y .
$$

where $j_{\mu}(x)=(\rho(x), \vec{j}(x))$ is the current operator and $A^{\mu}(x)$ is the vector potential. $B_{\mu \nu}(x, y)$ is the so called 'seagull' operator which results from the non-relativistic treatment of the hamiltonian [Fri75]. As explained in Ref. [Kra87] in a fully relativistic theory the 'seagull' operator corresponds to a sum over very high mass states which contain virtual nucleon-antinucleon pairs. These very complicated contributions are effectively taken into account by the 'seagull' operator.

If one neglects the second term in Eq. 2.1 and treats the first one in first order perturbation theory - which is well justified because the electromagnetic interaction is weak - one obtains the well known equations for the one-photon decay [Pel82] which are extensively used in nuclear physics. The corresponding Feynman diagram of the one-photon decay is shown in Fig. 2.1(a). 
In order to obtain the differential two-photon decay probability one has to treat the first term in Eq. 2.1 in second order perturbation theory and the second term in first order perturbation theory. The Feynman diagrams which contribute to the total two-photon decay amplitude are shown in Fig 2.1 (b-d). The so-called resonance amplitudes from the second order perturbation theory of the $j \cdot A$ term in Eq. 2.1 are displayed in $(c+d)$ and the 'seagull' amplitude, which is due to the first order perturbation theory of the $A^{2}$ term, is shown in (b).

Starting from Eq. (A.34.a) of Ref. [Kra87] one obtains, for a transition from a state with spin $I_{\mathrm{i}}$ to a state with spin $I_{\mathrm{f}}$ after integrating over all angles, expect the one between the emitted photons $\theta$, the differential two-photon decay probability [Mil13a]

$$
\begin{aligned}
\frac{d^{2} \Gamma_{\gamma \gamma}}{d \omega d(\cos \theta)}= & \frac{\omega \omega^{\prime}}{\left(2 I_{i}+1\right) \pi} \cdot \sum_{L L^{\prime} S S^{\prime} \bar{L} \bar{L}^{\prime} \bar{S} \bar{S}^{\prime} Q J} \sum_{\lambda \lambda^{\prime}} \\
& \left\{\lambda^{S+\bar{S}} \lambda^{\prime S^{\prime}+\bar{S}^{\prime}} \frac{\hat{J}^{2}}{\hat{\hat{L}^{\prime}}}(-)^{\lambda+\lambda^{\prime}+L^{\prime}+\bar{L}}(-)^{L^{\prime}+\bar{L}^{\prime}+Q}\right. \\
& \cdot\langle L-\lambda \bar{L} \lambda \mid Q 0\rangle \cdot\left\langle L^{\prime}-\lambda^{\prime} \bar{L}^{\prime} \lambda^{\prime} \mid Q 0\right\rangle \cdot U\left(\bar{L} J Q L^{\prime}, \bar{L}^{\prime} L\right) \\
& \left.\cdot P_{Q}(\cos \theta) \cdot P_{J}^{\prime}\left(S^{\prime} L^{\prime} S L, \omega^{\prime} \omega\right) \cdot P_{J}^{\prime}\left(\bar{S}^{\prime} \bar{L}^{\prime} \bar{S} \bar{L}, \omega^{\prime} \omega\right)\right\},
\end{aligned}
$$

with $\hat{x}=\sqrt{2 x+1}$. A rather similar expression is presented in Ref. [Gre63]. The equation is valid for the specialization that the spin of the initial nuclear state is randomly oriented and only the emission directions of the two photons are measured. Additionally Eq. 2.2 does not consider the 'seagull' amplitude. According to Ref. [Kra87] this part contributes only if both transitions are magnetic which is not the case for the considered transition in ${ }^{137} \mathrm{Ba}$ (see Sect 2.2).

The sums run over all unobserved quantum numbers. $L$ and $S(S=E=0$ and $S=M=1)$ are the multipolarities and the multipole characters of the virtual transitions, respectively. $J$ denotes the total angular momentum transfer carried by the two photons. The helicity is $\lambda=+1(-1)$ for positive (negative) helicity photons. The angular distribution is determined by the Legendre polynomials $P_{Q}(\cos \theta)$ and the $Q$ dependent coefficients. The energies of the two photons $\omega$ and $\omega^{\prime}$ have to satisfy the condition $\omega+\omega^{\prime}=\omega_{0}$ with $\omega_{0}$ being the transition energy. The angular momentum coupling coefficient $U$ is defined in Eq. A.1 of the appendix.

The nuclear structure information is contained in the so-called generalized polarizabilities $P_{J}$ which are given through [Kra87]

$$
\begin{aligned}
& P_{J}^{\prime}\left(\bar{S}^{\prime} L^{\prime} S L, \omega^{\prime} \omega\right)= \\
& \quad 2 \pi(-)^{S+S^{\prime}+L+L^{\prime}} \omega^{L} \omega^{L^{\prime}} \frac{\hat{I}_{f} \hat{L} \hat{\bar{L}}^{\prime}}{\hat{J}(2 L+1) ! ! \cdot\left(2 L^{\prime}+1\right) ! !} \cdot \sqrt{\frac{L+1}{L}} \cdot \sqrt{\frac{L^{\prime}+1}{L^{\prime}}} \\
& \quad \cdot \sum_{n}\left\{U\left(L L^{\prime} I_{i} I_{f}, J I_{n}\right) \cdot \frac{\left\langle I_{f}\left\|i^{L^{\prime}-S^{\prime}} M\left(S^{\prime} L^{\prime}\right)\right\| I_{n}\right\rangle\left\langle I_{n}\left\|i^{L-S} M(S L)\right\| I_{i}\right\rangle}{E_{i}-E_{n}-\omega}\right. \\
& \left.\quad+(-)^{L+L^{\prime}+J} \cdot U\left(L^{\prime} L I_{i} I_{f}, J I_{n}\right) \cdot \frac{\left\langle I_{f}\left\|i^{L-S} M(S L)\right\| I_{n}\right\rangle\left\langle I_{n}\left\|i^{L^{\prime}-S^{\prime}} M\left(S^{\prime} L^{\prime}\right)\right\| I_{i}\right\rangle}{E_{i}-E_{n}-\omega^{\prime}}\right\}
\end{aligned}
$$

The expression is symmetric under exchange of $\omega$ and $\omega^{\prime}$.

Furthermore the generalized polarizabilities have to obey the following parity selection rule

$$
(-1)^{L^{\prime}+S^{\prime}+L+S}=\pi_{i} \pi_{f},
$$


and the spin selection rule

$$
\begin{aligned}
& \left|I_{i}-I_{f}\right| \leq J \leq\left|I_{i}+I_{f}\right| \\
& \left|L^{\prime}-L\right| \leq J \leq\left|L^{\prime}+L\right| .
\end{aligned}
$$

In Eq. 2.3 the sum runs over all intermediate states $\left|I_{n}\right\rangle$ which can be connected by the transition operators $M(S L)$ to the initial and final states. Hence the two-photon decay probability is a new integral quantity of the nucleus allowing to study special structural properties of the nuclear system.

The total two-photon decay probability is obtained after integrating Eq. 2.2 over the relative angle of the two photons and their energies

$$
\Gamma_{\gamma \gamma}=\frac{1}{2} \int_{0}^{\omega_{0}} \int_{-1}^{+1} \frac{d^{2} \Gamma_{\gamma \gamma}}{d \omega d(\cos \theta)} d \omega d(\cos \theta) .
$$

The factor $\frac{1}{2}$ is necessary to avoid double counting due to the bosonic character of photons [Kra87].

In Ref. [Kra87] the usefulness of this quantity was demonstrated for the special case of the two-photon decay of a $\mathrm{O}_{2}^{+} \rightarrow \mathrm{O}_{1}^{+}$transition. It gave access to the electric dipole transition polarizability and magnetic dipole transition susceptibility which are not easily accessible through other experimental methods. The latter can be used to test the validity of $M 1$-sum rules and to obtain information on the quenching factor of the M1-strengths [Kra87].

According to Ref. [Kra87] the magnetic dipole transition susceptibility is defined as

$$
\chi^{12}=\chi_{P}^{12}+\chi_{D}^{12}
$$

where $\chi_{P}^{12}$ and $\chi_{D}^{12}$ are the paramagnetic and diamagnetic transition susceptibilities (for a definition see Ref. [Kra87]). The paramagnetic transition susceptibility is closely related to the diagonal paramagnetic susceptibility of the ground state [Kra87]. Which is given through

$$
\chi_{P}=\frac{4}{9} \pi \cdot 2 \sum_{n} \frac{\left|\left\langle 0_{1}^{+} \| M(M 1)|| 1_{n}^{-}\right\rangle\right|^{2}}{E_{n}} .
$$

$\chi_{P}$ can be determined in inelastic electron scattering or in inelastic proton scattering experiments under zero degrees [Tam09]. If one is able to measure $\chi^{12}$ with sufficient accuracy one can combine the results of both experiments to determine $\chi_{D}^{12}$ for the first time. This would provide a direct test of the 'seagull' operator [Kra87] which is closely related to $\chi_{D}^{12}$ and give valuable information on the importance of mesonic degrees of freedom.

Although the discussion in Ref. [Kra87] is limited to the $0_{2}^{+} \rightarrow 0_{1}^{+}$case one can do the same kind of investigations for other transitions like $2_{1}^{+} \rightarrow 0_{1}^{+}$. Clearly, this requires that one finds experimentally a way to deal with the - then not forbidden - one-photon decay.

The diagonal electric dipole polarizability of the ground state is defined as [Rei10, Kra87]

$$
\alpha_{E 1}=\frac{4}{9} \pi \cdot 2 \sum_{n} \frac{\left|\left\langle 0_{1}^{+}\|i M(E 1)\| 1_{n}^{-}\right\rangle\right|^{2}}{E_{n}} .
$$


It is used e.g. to restrict the parameters of the equation of state [Tew13] or to determine the neutron skin thickness [Tam11]. It is thinkable that one uses the electric dipole transition polarizability obtained from the two-photon decay probability for the same investigations. Whether this is possible has to be answered by a detailed theoretical analysis. One problem might be that the contributions of the various intermediate states cancel in the sum in Eq. 2.3. Such a cancellation is not possible for the diagonal electric dipole polarizability.

In general the idea to obtain a similar integral quantity like the diagonal electric dipole polarizability and the diagonal paramagnetic dipole susceptibility just from a transition between two low-lying states - as it is the case for the two-photon decay - is very appealing. To identify all states of one multipolarity and parity over the full energy range is experimentally very demanding and one can easily miss a part of the strength. Due to the sum over all intermediate states in Eq. 2.3 this is not possible in case of the two-photon decay probability.

\subsection{The case of ${ }^{137} \mathrm{Ba}$}

In the following, the general results of the last section are applied to the case of ${ }^{137} \mathrm{Ba}$. In order to keep the discussion transparent a simple nuclear structure model is employed.

The following results are based on the work of D.J. Millener who calculated the relevant observables for the two-photon decay in the independent particle model. The discussion follows closely his article in Ref. [Mil13a].

The $\beta^{-}$-decay of ${ }^{137} \mathrm{Cs}$ populates the $1 h_{11 / 2}^{-}$state in ${ }^{137} \mathrm{Ba}$ which decays via a $M 4$-transition and the emission of a single photon to the $2 d_{3 / 2}^{+}$ground state. The two-photon decay proceeds through higher-lying intermediate states. According to the spin selection rule of Eq. 2.5 the allowed values for the total angular momentum transfer are $J=4,5,6,7$. In a very good approximation one can restrict the sum in Eq. 2.2 to $J=4$ [Mil14]. Furthermore the discussion is limited to the case where the multipolarities $L, L^{\prime}$ sum to $J=4$, i.e. $L+L^{\prime}=4$. Hence the considered transitions are $E 1+M 3, M 1+E 3$ and $M 2+E 2$. The quality of this assumption has still to be verified e.g. an appreciable contribution of the $E 2+E 3$ term in Eq. 2.2 cannot be ruled out [Mil14].

In the following the two-photon decay probability is estimated in the independent particle shell model. The nucleus ${ }^{137} \mathrm{Ba}$ is one neutron away from the $N=82$ shell closure. The $2 d_{3 / 2}^{+}$ground state as well as the $1 h_{11 / 2}^{-}$state at $662 \mathrm{keV}$ are assumed to be neutron hole states.

Due to the approximations made above the intermediate states have to have angular momentum $j=5 / 2,7 / 2$ or $9 / 2$. The configuration of the $1 h_{11 / 2}^{-}$state is schematically shown on the right-hand side of Fig. 2.2. Within the same major shell the neutron hole can be excited to the to the $2 d_{5 / 2}^{+}$and $1 g_{7 / 2}^{-}$single particle states. In the next major shell there is the possibility to go to the $1 g_{9 / 2}^{+}$and $1 f_{5 / 2}^{-}$single particle states. On the other hand an excitation of a neutron from the $1 h_{11 / 2}^{-}$single-particle state above the $N=82$ shell closure to e.g. the $2 f_{7 / 2}^{-}$single particle state is not allowed in this simple model, since it is not possible to obtain again the $2 d_{3 / 2}^{+}$ground state configuration in one step. Some of the possible paths for the two-photon decay in this simple model are presented in Fig. 2.2 with the corresponding multipolarities.

The denominator of Eq. 2.3 depends on the energy difference between the $1 h_{11 / 2}^{-}$single particle state and the corresponding intermediate single particle state. The $1 g_{9 / 2}^{+}$and $1 f_{5 / 2}^{-}$hole states are higher in energy than the $2 d_{5 / 2}^{+}$and $1 g_{7 / 2}^{-}$hole states since they belong to the next 

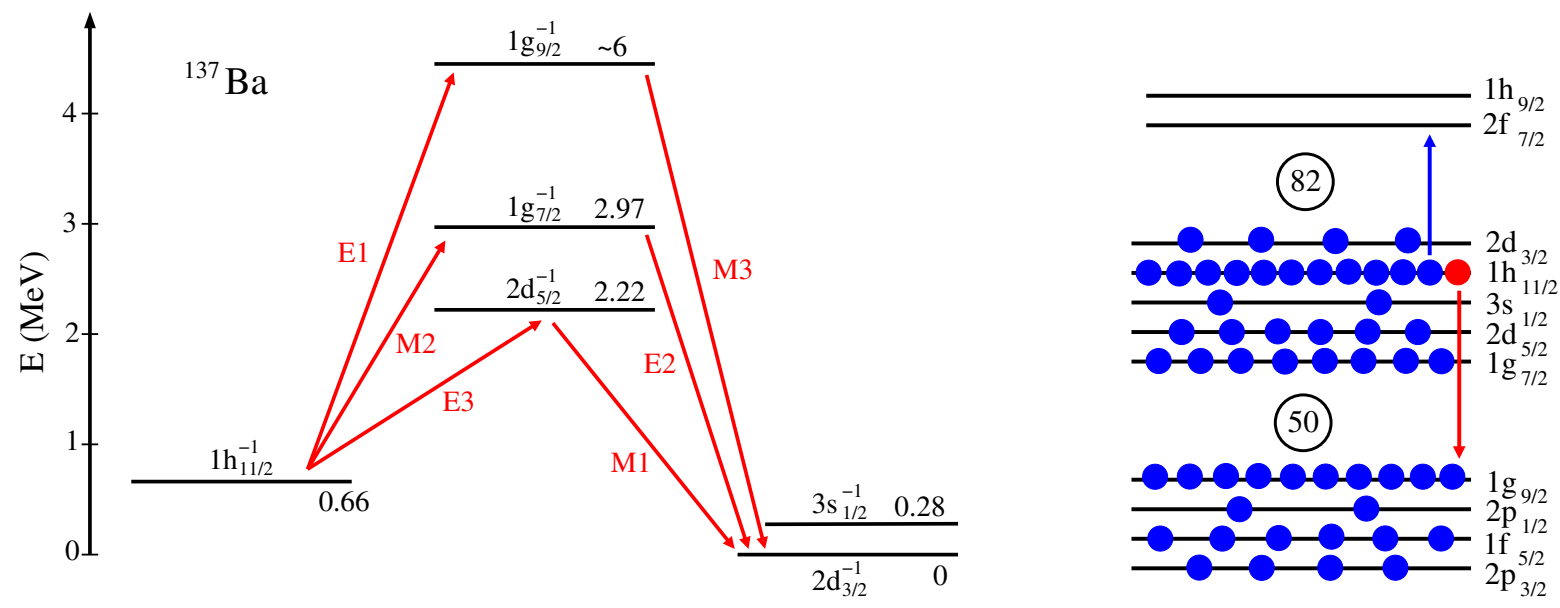

Figure 2.2: Possible decay paths of the two-photon decay from the $1 h_{11 / 2}^{-}$neutron hole state to the $2 d_{3 / 2}^{+}$ground state through intermediate neutron hole states (left-hand side). On the right hand side the neutron configuration in the independent particle model of the $1 h_{11 / 2}^{-}$state is shown. A transition through the $1 g_{9 / 2}^{+}$state is allowed (red arrow). On the other hand an excitation from a neutron to the $2 f_{7 / 2}^{-}$-state is not possible (blue arrow), since there is no one-step deexcitation back to the $2 d_{3 / 2}^{+}$ground state. Figure on the left hand side is taken, and slightly changed, from Ref. [Mil13a].

major shell. Hence, to a good approximation, one can neglect the $E 1+M 3$ paths in Fig. 2.2 and consider the $M 2+E 2$ and $M 1+E 3$ multipole pairs only.

After integrating Eq. 2.2 over $d(\cos \theta)$ one obtains - under the made approximations - for one pair of multipoles

$$
\begin{aligned}
\frac{d \Gamma_{\gamma \gamma}}{d \omega} & =\frac{2 I_{f}+1}{2 I_{i}+1} 32 \pi \frac{L+1}{L} \frac{L^{\prime}+1}{L^{\prime}}\left[\frac{1}{(2 L+1) ! !\left(2 L^{\prime}+1\right) ! !}\right]^{2} \\
& \cdot \omega \omega^{\prime}\left[\left\{\sum_{n} \frac{\omega^{L} \omega^{\prime L^{\prime}}\left\langle I_{f}\left\|i^{L^{\prime}-S^{\prime}} M\left(S^{\prime} L^{\prime}\right)\right\| I_{n}\right\rangle\left\langle I_{n}\left\|i^{L-S} M(S L)\right\| I_{i}\right\rangle}{E_{i}-E_{n}-\omega}\right\}^{2}\right. \\
& \left.+\left\{\sum_{n} \frac{\omega^{L^{\prime}} \omega^{\prime L}\left\langle I_{f}\left\|i^{L^{\prime}-S^{\prime}} M\left(S^{\prime} L^{\prime}\right)\right\| I_{n}\right\rangle\left\langle I_{n}\left\|i^{L-S} M(S L)\right\| I_{i}\right\rangle}{E_{i}-E_{n}-\omega^{\prime}}\right\}^{2}\right]
\end{aligned}
$$

In principle one can integrate Eq. 2.10 numerically over $d \omega$ to obtain the two-photon decay probability $\Gamma_{\gamma \gamma}$. However, in order to obtain a deeper insight in the two-photon decay the denominator is assumed to be independent of $\omega: \Delta E=E_{i}-E_{n}-\omega_{0} / 2$ which allows to write Eq. 2.10 as

$$
\frac{d \Gamma_{\gamma \gamma}}{d \omega} \sim \frac{\left\langle I_{f}\left\|i^{L^{\prime}-S^{\prime}} M\left(S^{\prime} L^{\prime}\right)\right\| I_{n}\right\rangle^{2}\left\langle I_{n}\left\|i^{L-S} M(S L)\right\| I_{i}\right\rangle^{2}}{\Delta E^{2}}\left[\omega^{2 L+1} \omega^{2 L^{\prime}+1}+\omega^{2 L^{\prime}+1} \omega^{\prime 2 L+1}\right] .
$$

This approximation is valid if $E_{i}-E_{n}$ is large compared to $\omega_{0}$. If one integrates Eq. 2.11 over $d \omega$ and divides the result with the expression for the $M 4$-one-photon decay radiation width one obtains simple equations for the branching ratios in the $M 2+E 2$ case [Mil13a]

$$
\frac{\Gamma_{\gamma \gamma}}{\Gamma_{\gamma}}=4.58 \cdot 10^{-2} \cdot \frac{\omega_{0}}{\Delta E} \alpha \frac{B\left(M 2 ; 1 h_{11 / 2}^{-} \rightarrow 1 g_{7 / 2}^{+}\right) \cdot B\left(E 2 ; 1 g_{7 / 2}^{-} \rightarrow 2 d_{3 / 2}^{+}\right)}{B\left(M 4 ; 1 h_{11 / 2}^{-} \rightarrow 2 d_{3 / 2}^{+}\right)},
$$


and for the $M 1+E 3$ case

$$
\frac{\Gamma_{\gamma \gamma}}{\Gamma_{\gamma}}=5.82 \cdot 10^{-2} \cdot \frac{\omega_{0}}{\Delta E} \alpha \frac{B\left(E 3 ; 1 h_{11 / 2}^{-} \rightarrow 2 d_{5 / 2}^{+}\right) \cdot B\left(M 1 ; 2 d_{5 / 2}^{+} \rightarrow 2 d_{3 / 2}^{+}\right)}{B\left(M 4 ; 1 h_{11 / 2}^{-} \rightarrow 2 d_{3 / 2}^{+}\right)} .
$$

In principle the branching ratio is given through $\Gamma_{\gamma \gamma} /\left(\Gamma_{\gamma}+\Gamma_{\gamma \gamma}+\ldots\right)$. However other contributions than the one-photon decay are neglected in the denominator, since they are expected to be several orders of magnitude smaller. In order to determine the quadrupole-quadrupole and dipole-octupole branching ratios the energy differences $\Delta \mathrm{E}$ between the $1 h_{11 / 2}^{-}$single particle states and the $2 d_{5 / 2}^{+}$and $1 g_{7 / 2}^{-}$single particle states have to be known. The energies of the $1 h_{11 / 2}^{-}$and $1 g_{7 / 2}^{-}$single particle states can be determined approximately from ${ }^{138} \mathrm{Ba}(p, d){ }^{137} \mathrm{Ba}$ data [Jol71] and amount to $0.66 \mathrm{MeV}$ and $2.97 \mathrm{MeV}$ respectively. The energy of the $2 d_{5 / 2}^{+}$orbital at $2.22 \mathrm{MeV}$ is taken from Ref. [Isa02].

The $B\left(M 4: 1 h_{11 / 2}^{-} \rightarrow 2 d_{3 / 2}^{+}\right)$-value of $8.89 \cdot 10^{4} \mu_{N}^{2} \mathrm{fm}^{6}$ [Mil13a] is taken from experiment. The other transition strengths are calculated using harmonic oscillator wave functions. The effective charges are $e_{n}=1.0$ for $E 2$ - and E3-transitions. For the $M 1$-transition the effective operator of Ref. [Bro05] was used and for the $M 2$-transition the spin g-factor was assumed to be $g_{\mathrm{s}}^{\text {eff }}=0.7 g_{\mathrm{s}}^{\text {free }}$. More details are given in Ref. [Mil13a].

The resulting branching ratios in this simple independent particle model are given in Tab. 2.1. The two-photon decay branching ratio is small and amounts to $2.06 \cdot 10^{-6}$. It is experimentally very demanding to measure a decay mode which has such a small branching ratio. Furthermore the contributions of the quadrupole-quadrupole and dipole-octupole multipole pairs are predicted to be similar in size.

\begin{tabular}{c|c|c|c}
\hline \hline & $M 2+E 2$ & $M 1+E 3$ & full \\
\hline IPM & $1.28 \cdot 10^{-6}$ & $0.78 \cdot 10^{-6}$ & $2.06 \cdot 10^{-6}$ \\
\hline \hline
\end{tabular}

Table 2.1: Two-photon decay branching ratio $\Gamma_{\gamma \gamma} / \Gamma_{\gamma}$ calculated in the independent particle model (IPM) according to Eqs. 2.12 and 2.13.

In order to understand the two-photon decay it is desirable to obtain information on the the contributing multipole pairs. From the $Q$ dependent factors in Eq. 2.2 one can obtain the angular distribution of each multipole pair. For the quadrupole-quadrupole pair the angular distribution is given through

$$
W(\theta)=1+\frac{5}{49} P_{2}(\cos \theta)+\frac{40}{441} P_{4}(\cos \theta)
$$

and for the dipole-octupole case

$$
W(\theta)=1-\frac{1}{8} P_{2}(\cos \theta)
$$

The angular distributions for pure multipole pairs and fixed $\omega$ are presented on the left hand side of Fig. 2.3.

The variation of $W(\theta)$ with the relative angle between both emitted photons is rather weak. However this angular distribution can be more pronounced for other multipole pairs. Furthermore interference effects can alter the angular distribution significantly. 

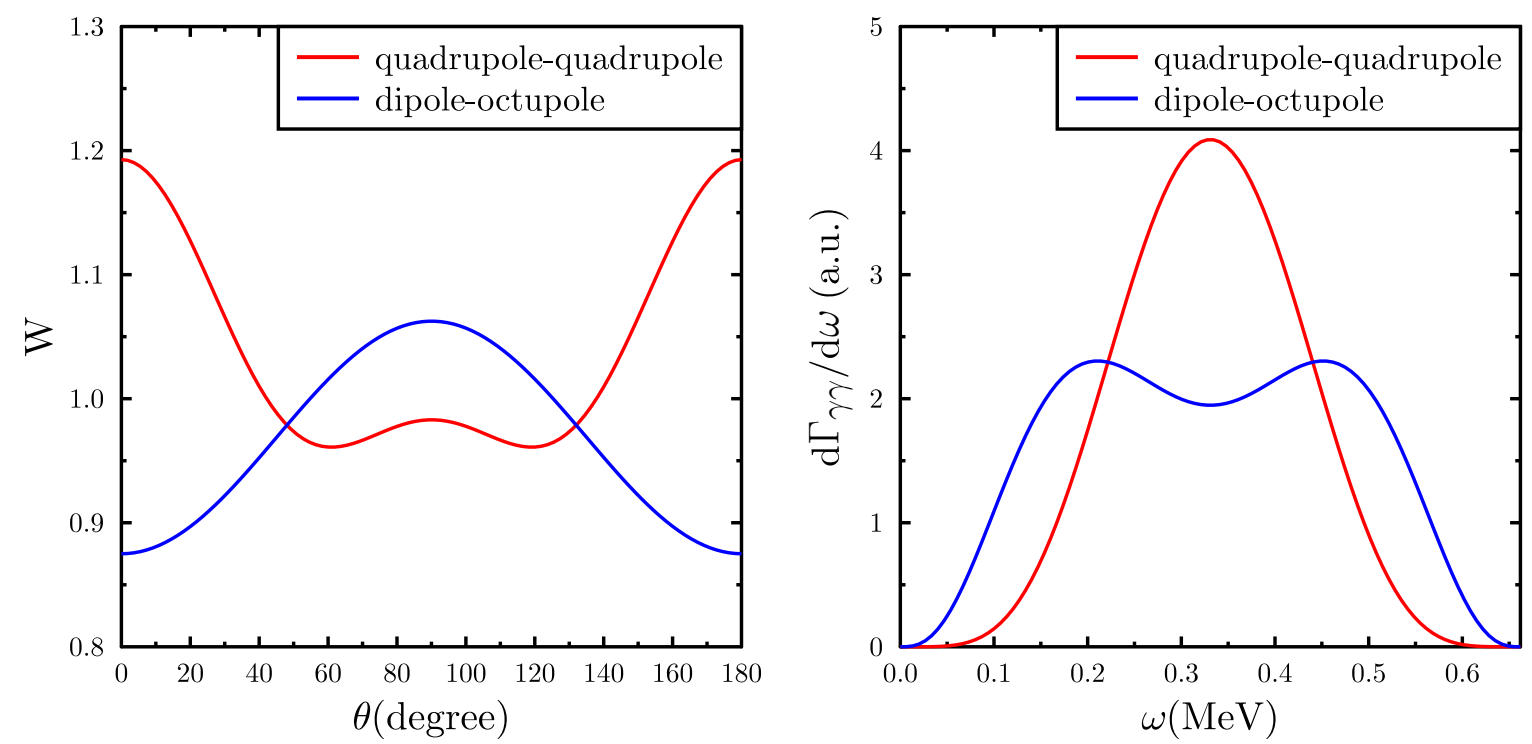

Figure 2.3: The dependence of the two-photon decay probability on the relative angle between the two emitted photons (left-hand side) and on the photon energy $\omega$ (right-hand side). The distributions are shown for the quadrupole-quadrupole (red) and dipoleoctupole (blue) multipole pairs.

Another way to obtain information on multipole pairs is to investigate the dependence of $\Gamma_{\gamma \gamma} / d \omega$ on the photon energy $\omega$. According to Eq. $2.11 d \Gamma_{\gamma \gamma} / d \omega \sim \omega^{5} \omega^{15}$ in case of quadrupole-quadrupole and $d \Gamma_{\gamma \gamma} / d \omega \sim \omega^{3} \omega^{77}+\omega^{7} \omega^{\prime 3}$ in case of dipole-octupole. Both distributions are shown on the right hand side of Fig. 2.3 assuming $\omega+\omega^{\prime}=662 \mathrm{keV}$. In Eq. 2.11 the denominator is assumed to be independent of $\omega$. If the energy difference of the corresponding single particle states $\Delta E$ is small (relative to $\omega_{0}$ ) this assumption is not justified and the distributions in Fig. 2.3 will slightly change [Mil14].

The considerations in the independent particle model allow to draw some general conclusions on the design of an experiment to measure the two-photon decay branch.

- The two-photon decay branching ratio is of the order of $\sim 2 \cdot 10^{-6}$ i.e. background and Compton scattering of photons stemming from the one-photon decay (see Sect. 3.1) have to be suppressed sufficiently to measure a decay branch of this order of magnitude.

- It is important to obtain information on the angular distribution and energy sharing function to decide which multipole pairs contribute to the two-photon decay.

- The present experiment was conducted with five $\mathrm{LaBr}_{3}$-detectors with a distance of $22 \mathrm{~cm}$ to the source. The source strength was $606 \mathrm{kBq}$ (October $5^{\text {th }}$ 2013). With a simulation of the full energy efficiency of the $\mathrm{LaBr}_{3}$-detectors one obtains a rate of 3.4 two-photon events per detector pair and day. For simplicity the full energy efficiency at $331 \mathrm{keV}$ was used for this rate estimate taken from a Monte-Carlo simulation.

The values in Tab. 2.1 are just rough estimates for the two-photon decay probabilities. The used model is simple and is restricted to two decay paths. A calculation in a more advanced nuclear structure model like the Quasi-Particle-Phonon model (see Sect. 5.3) is clearly required. This would also allow to study the influence of the giant resonances on the two-photon decay 
probability which can significantly alter the results in terms of the contribution multipole pairs as well as the absolute value of the two-photon decay branching ratio. 


\section{Experimental setup of the two-photon decay experiment}

In order to measure the two-photon decay - if the one-photon decay is allowed - one has to deal with several challenging experimental problems which are discussed in this chapter. The problems of 'direct' and 'sequential' Compton scattering are outlined in Sect. 3.1 and a detector arrangement is presented which suppresses both unwanted effects. Since the rate of the two-photon decay amounts to a few events per day and detector pair only, it is important to investigate the background which is present even without a ${ }^{137} \mathrm{Cs}$ source. In Sect. 3.2 it is shown, that the ratio of the background rate to the expected two-photon rate is sufficiently small to perform the experiment. A method to further suppress this background is presented. In Sect. 3.3 the problem of time random coincidences and implications for the choice of the source strength and for the distance from detector to source are discussed. In the last section of this chapter the general data taking conditions and the electronic setup are presented.

\subsection{Lead shield and choice of detector arrangement}

The two-photon branching ratio of the $1 h_{11 / 2}^{-} \rightarrow 2 d_{3 / 2}^{-}$-transition in ${ }^{137} \mathrm{Ba}$ is predicted to be $2.06 \cdot 10^{-6}$ i.e. the one-photon decay to the ground state is six orders of magnitude more likely. This huge difference causes experimental problems to separate events due to the two-photon decay, from events which are caused by second and third order processes of the photon stemming from the one-photon decay. The two main problems - 'direct' and 'sequential' Compton scattering of $662 \mathrm{keV}$ photons - are schematically shown on the left-hand side of Fig. 3.1.

The photon of the one-photon decay with an energy of $662 \mathrm{keV}$ can deposit only a part of its energy in detector 1 and is then Compton scattered in the direction of the second detector. If this photon deposits its full remaining energy in detector 2, the sum of both energies, measured in both detectors equals $662 \mathrm{keV}$. These events cannot be easily separated from events of the two-photon decay which also fulfill the condition that the sum energy equals $662 \mathrm{keV}$. $\mathrm{LaBr}_{3}$ detectors have an excellent time resolution of the order of $\sim 1$ ns (see Sect. 4.2). Nevertheless the time resolution is not good enough to use the additional distance $(20-30 \mathrm{~cm})$ traveled by the Compton scattered photon to identify and suppress these events. It is possible to increase this distance by changing the distance between detector and source, this, however, would reduce the efficiency of each detector to an unacceptable value.

Therefore, in the present experiment, it was decided to use lead - which is an excellent absorber of photons due to its high density and $Z$ value - to block Compton scattered events. In order to determine the necessary lead thickness the setup, shown in Fig. 3.1 was investigated with a GEANT4 simulation [Ago03] (without material ' $A$ ' in Fig. 3.1). The distance between detector and source $(22 \mathrm{~cm})$ and the angle between both detectors $\left(\theta=72^{\circ}\right)$ is similar to the setup used in the actual experiment. In the simulation $\mathrm{N}_{\gamma}=10^{9}$ photons with an energy of $662 \mathrm{keV}$ were shot randomly distributed on detector 1 and the number of events were recorded where 
both detectors were hit and their sum energy $E_{1}+E_{2}$ lies in the energy window from 642 to $682 \mathrm{keV}$. The thickness $\mathrm{d}$ of the lead block was varied systematically from 0 to $22 \mathrm{~mm}$ in $2 \mathrm{~mm}$ steps. The resulting number of events fulfilling these conditions - shown on the right-hand side of Fig. 3.1 - drop almost exponentially with the thickness $d$. For $d=22 \mathrm{~mm}$ only $\sim 5$ events are able to pass the lead block without interaction. In the present experiment a ${ }^{137} \mathrm{Cs}$ source with a strength of $487 \cdot 10^{3} 662 \mathrm{keV}$-photons per second was used. Hence the number of photons used in the simulation is similar to the number of photons impinging on each detector in 3.24 days. Since the total measurement time amounts to $\sim 50$ days it would have been necessary to carry out the simulation with $\sim 3 \cdot 10^{10}$ events which is not in reach. However the minimum lead thickness chosen in experiment was $\sim 8 \mathrm{~cm}$ which is a rather conservative choice considering the rapid drop of the number of Compton scattered events in Fig. 3.1.
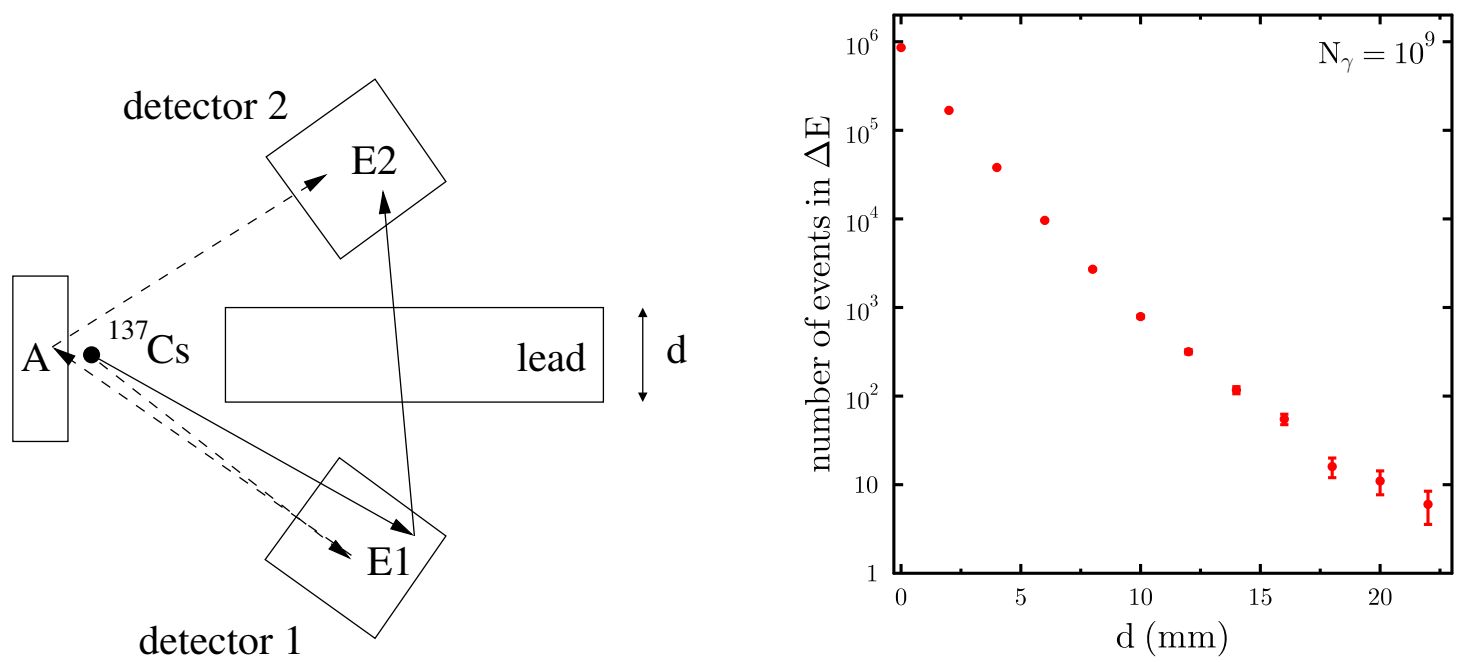

Figure 3.1: Illustrated are the two main problems which are due to the presence of the onephoton decay (left-hand side). A gamma-ray with an energy of $662 \mathrm{keV}$ deposits the energy $E_{1}$ in detector 1 and is then scattered into detector 2 where it deposits the remaining part of its energy (solid black arrows). The sum energy $E_{1}+E_{2}$ equals $662 \mathrm{keV}$ i.e. it is not distinguishable from a two-photon event. In order to suppress Compton scattered events a lead shield was positioned between both detectors. The second problem is 'sequential' Compton scattering i.e. the $662 \mathrm{keV}$ gamma-ray is scattered by $\sim 180^{\circ}$ and is then scattered again on material ' $A$ ' into detector 2 . On the right hand are shown the suppression factors of the lead shield for 'direct' Compton scattered events in dependence of its thickness $d$. The simulation is performed with $\mathrm{N}_{\gamma}=10^{9}$ gamma-rays with an energy of $662 \mathrm{keV}$. For more details see text.

The experimental setup is presented in Fig. 3.3. Five $\mathrm{LaBr}_{3}$-detectors were mounted on the support structure of the $\mathrm{LaBr}_{3}$-detector ball. In total 10 detector pairs can be formed, five with an relative angle of $72^{\circ}$ (e.g. detectors 1 and 2) and five with an angle of $144^{\circ}$ (e.g. detectors 1 and 3). The path between each detector pair is blocked with customized lead bricks. The lines up to where it is necessary to put lead are highlighted with dashed red lines. Care was taken that there are no open slits between neighboring lead bricks. The minimum lead thickness between pairs with an angle of $72^{\circ}$ were $12.5 \mathrm{~cm}$ and $\sim 8 \mathrm{~cm}$ between pairs of $144^{\circ}$.

The second major problem in this experiment is 'sequential' Compton scattering. As shown in Fig. 3.1 a photon stemming from the one-photon decay interacts with detector 1 and is 


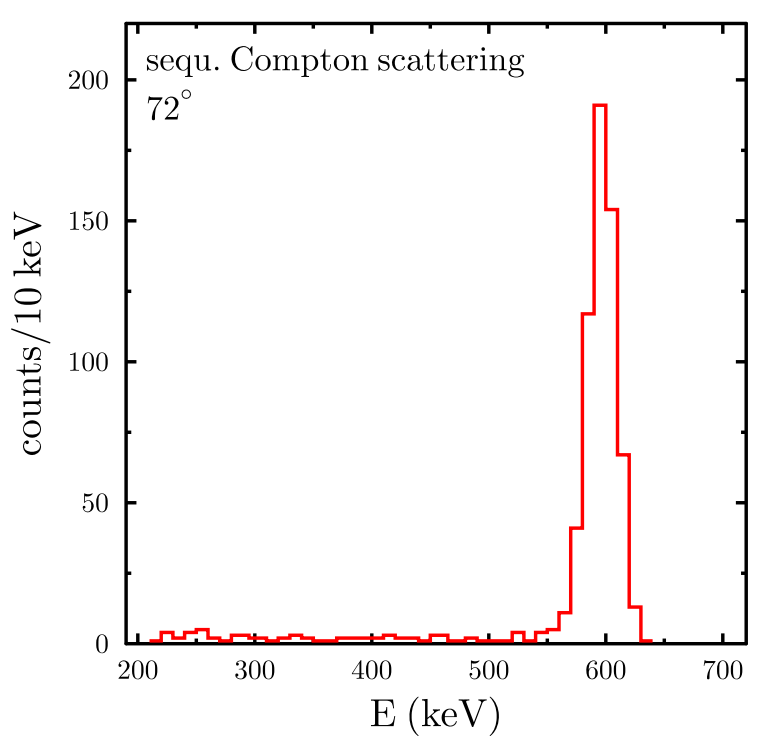

Figure 3.2: The energy spectrum of detector 2 in case of 'sequential' Compton scattering on material ' $A$ ' (see Fig. 3.1).

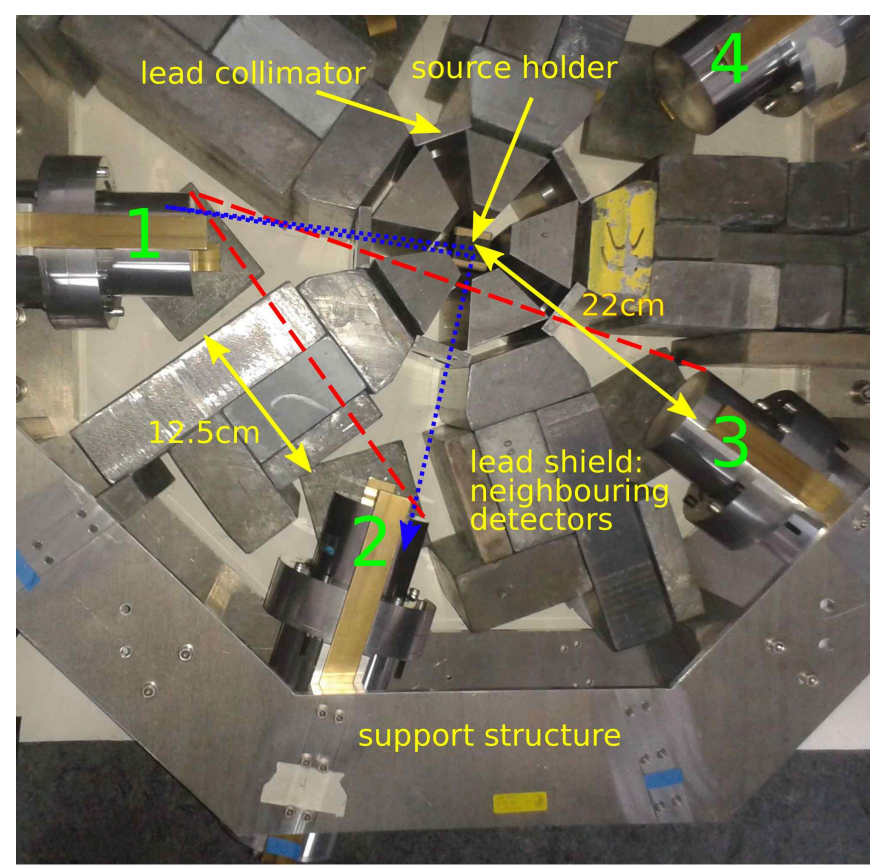

Figure 3.3: Experimental setup of the two-photon experiment. The detectors were separated by customized lead bricks. The dotted blue lines show a possible path for 'sequential' Compton scattering between detectors 1 and 2 . The dashed red line is the geometrical limit where it is necessary to put lead bricks in order to prevent 'direct' Compton scattering between the corresponding detector pair.

scattered in the opposite direction by an angle of $\sim 180^{\circ}$. Then it interacts with material ' $A$ ' which is nearby the source - e.g. source holder, source, air, lead bricks - and is scattered in the direction of detector 2 where it deposits its full remaining energy. The resulting sum energy spectrum - which was simulated with $\mathrm{N}_{\gamma}=10^{9}$ events - is displayed in Fig. 3.2. The thickness $d$ of the lead brick between the detectors is large enough to prevent any 'direct' Compton scattering 
i.e. the spectrum shown in Fig. 3.2 is solely due to 'sequential' Compton scattering. Clearly the spectrum is dominated by a peak with an energy of $\sim 600 \mathrm{keV}$. Its origin can be understood considering the Compton formula given by

$$
E_{\gamma}^{\prime}=\frac{E_{\gamma}}{1+\frac{E_{\gamma}}{m_{e} c^{2}}(1-\cos \phi)} .
$$

A $662 \mathrm{keV}$ photon which is scattered by an angle of $\phi=\sim 180^{\circ}$ has, after the scattering process, an energy of $184 \mathrm{keV}$. This photon is then again scattered by an angle of $\sim 72^{\circ}$ and has a final energy of $125 \mathrm{keV}$ which is deposited in detector 2 . The sum energy of detector 1 and 2 amounts to $478 \mathrm{keV}+125 \mathrm{keV}=603 \mathrm{keV}$ and is approximately equal to the centroid energy of the peak in Fig. 3.2.

In principle this peak is well separated in energy from the expected two-photon peak at $662 \mathrm{keV}$. However, if the strength of 'sequential' Compton scattering is several orders of magnitude higher than the two-photon decay rate, it might significantly complicate or prevent the determination of the two-photon branching ratio.

The situation is worse for detector pairs with an relative angle of $144^{\circ}$. According to Eq. 3.1 the energy of the 'sequential' Compton scattering peak is $650 \mathrm{keV}$ i.e. very close to $662 \mathrm{keV}$. Especially in this case methods to suppress this effect are necessary.

First of all one can use energy gates around $478 \mathrm{keV}$ and $125 \mathrm{keV}$ in case of detector pairs with a relative angle of $72^{\circ}$ and around $478 \mathrm{keV}$ and $172 \mathrm{keV}$ in case of $144^{\circ}$. Events which fall within these gates are not considered in the further analysis. This is possible because of the lead collimator of each detector which guarantees that Compton scattering occurs in a narrow angular range (and hence energy range). Secondly the good time resolution of $\mathrm{LaBr}_{3}$-detectors can be used to set a narrow time gate on the two-photon coincidence peak. A 'sequential' Compton scattered photon travels additional $\sim 45 \mathrm{~cm}$, i.e. the time coincidence peak is shifted by $\sim 1.5$ ns relative to the two-photon coincidence peak. Although $\sim 1.5$ ns are not enough the allow a clean separation between both processes, it is still possible to suppress 'sequential' Compton scattering.

Thirdly cardboard was used as material for the target holder. The low density of the material reduces the probability for Compton scattering. Indeed in the beginning of the two-photon decay experiment an aluminum bar was used as a target holder which resulted in a massive 'sequential' Compton scattering peak. Lastly a special lead collimator (see Fig. 3.3) was positioned in front of each detector which let only the solid angle of the detector open and hence blocks a part of the 'sequential' Compton scattered events. The combination of all four methods reduced the influence of 'sequential' Compton scattering to an acceptable amount.

\subsection{Background conditions}

Since the rate of the two-photon decay amounts to a few events per day only, it is important to consider the background being present even without a radioactive source. In order to study its influence a background measurement was conducted with the setup presented in the last section. The measurement time was 3.83 days. Only background events were of interest where two detectors fired in coincidence. Furthermore the energy in each detector was required to be higher than $100 \mathrm{keV}$ and the condition $\left|E_{1}-E_{2}\right|<250 \mathrm{keV}$ has to be fulfilled. Without these 
additional energy conditions the background would increase drastically. The resulting time spectra are shown on the right hand side of Fig. 3.5 for relative detector angles of $72^{\circ}$ (solid red line) and $144^{\circ}$ (solid green line). Each spectrum is the sum spectrum of the corresponding five detector pairs which were time aligned with a ${ }^{60} \mathrm{Co}$ source measurement beforehand. Clearly, even without a source a sizable background is present in the laboratory causing a broad time coincidence peak with a width of $\sim 5$ ns (FWHM).

Important for the two-photon decay experiment is the number of background events in the energy range of $\sim 652$ to $\sim 672 \mathrm{keV}$. A time condition was applied considering only events with a time difference between -1.2 and $1.2 \mathrm{~ns}$. This time gate is the same like the one used in the actual experiment (see Sect. 4.2). The resulting sum energy spectra are presented on the left-hand side of Fig. 3.5. The background increases for smaller $E_{1}+E_{2}$ values especially for the angle group of $72^{\circ}$. In the energy range of 652 to $672 \mathrm{keV}$ the number of background events per day and detector pair amounts to 6.6(7) events for $72^{\circ}$ and $1.0(3)$ events for $144^{\circ}$. The background is smooth in energy and can in principle be separated from the two-photon peak. However the accuracy of the measurement is reduced if the uncertainty of the background meaning the square root of the number of counts in the energy range from 652 to $672 \mathrm{keV}$ has a similar value than the number of two-photon events. Since the expected number of twophoton events is 3.4 per day and detector pair, this is indeed the case for both angles. Clearly, this can be compensated by a long measurement time.
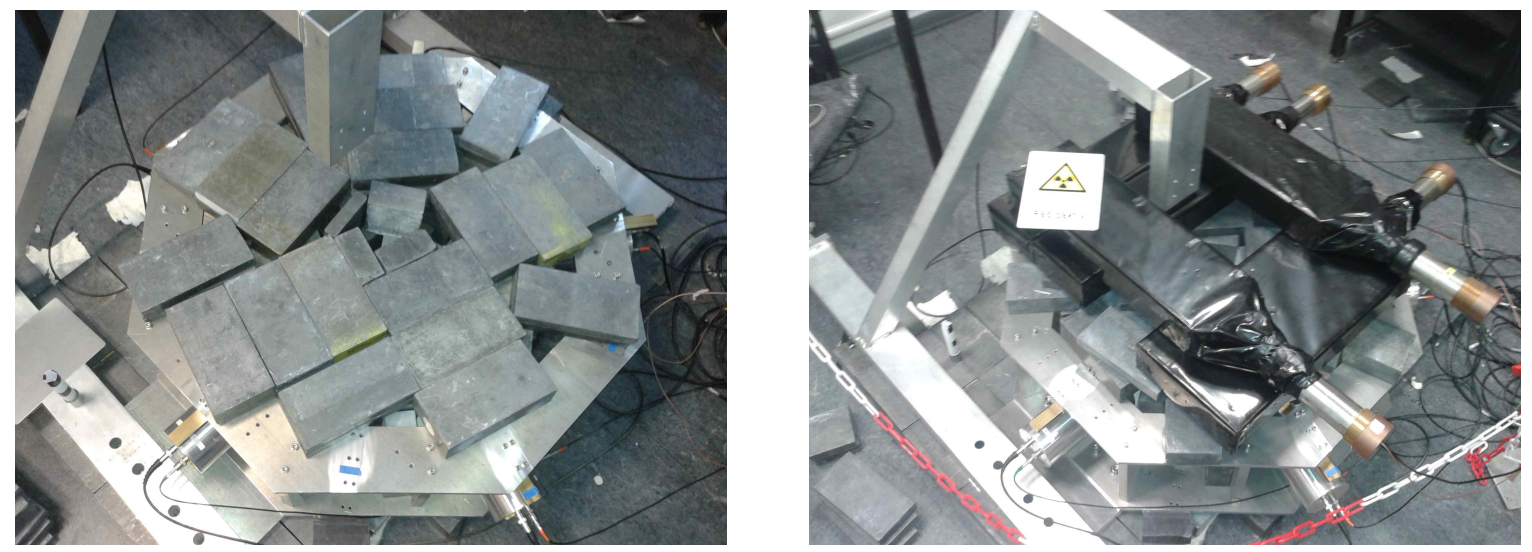

Figure 3.4: The setup shown in Fig. 3.3 was covered with a 5-10 cm thick layer of lead (left-hand side). Five plastic scintillators were positioned on top of it to veto background events (right-hand side).

In order to reduce the background five plastic scintillators were positioned on top of the experimental setup as shown on the right hand side of Fig. 3.4. The signals of the plastic scintillators were used as veto i.e. whenever two $\mathrm{LaBr}_{3}$-detectors and one or more plastic scintillators fired in coincidence the corresponding events were not considered in the further analysis. This has proven to be an efficient way to suppress background events. The corresponding time and energy spectra are shown in Fig. 3.5 for $72^{\circ}$ (solid blue lines) and $144^{\circ}$ (solid black lines). In the energy window from 652 to $672 \mathrm{keV}$ the number of events per day and detector pair dropped by factor of $\sim 1.5$ to $4.3(5)$ and $0.7(2)$ for relative detector angles of $72^{\circ}$ and $144^{\circ}$ respectively. The results of the background measurements are summarized in Tab. 3.1.

Despite efforts to understand the background, it was not possible to draw firm conclusions about its origin. Possible sources are cosmic rays and their decay products, the internal radioac- 

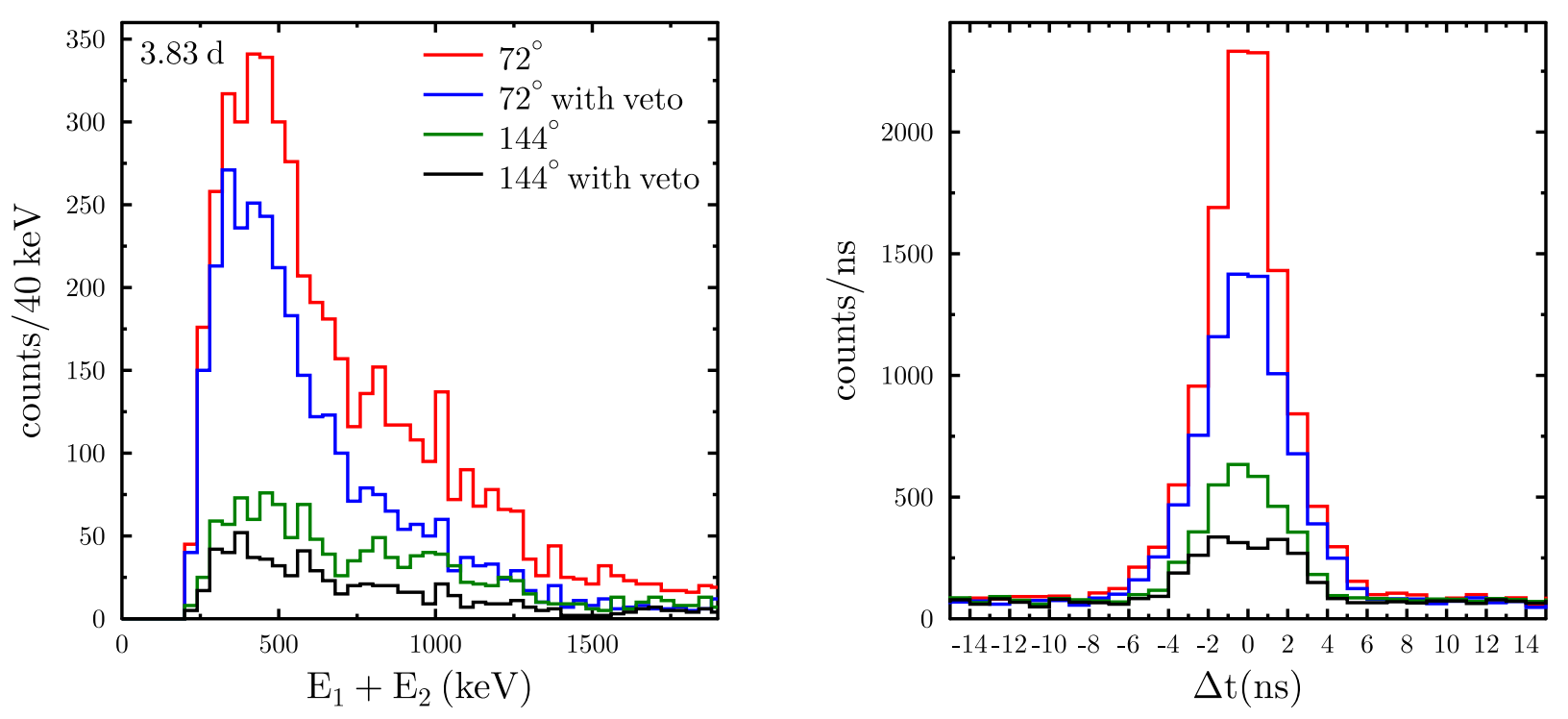

Figure 3.5: Background sum energy (left-hand side) and time spectra (right-hand side) with and without veto of the plastic scintillators for both angle groups. The veto helps significantly to suppress background events. Furthermore the background under significantly smaller than under $72^{\circ}$. Its origin is not understood.

tivity of $\mathrm{LaBr}_{3}$, which includes a gamma-ray of $\sim 1440 \mathrm{keV}$ which penetrates lead more easily, or a radioactive decay in one of the lead bricks. Since the veto of the plastic scintillators provides an improvement, it seems likely that at least a part of the background stems from cosmic rays which dominantly come from above. Interestingly the background scales with the distance between both detectors because the background rate for detectors with an relative angle of $72^{\circ}$ is a factor of $\sim 6$ higher than for pairs with an angle of $144^{\circ}$. In order to understand the origin of the background and to find a better way to suppress it, a long measurement with germanium detectors might be useful. Their excellent energy resolution allows probably to identify discrete states in the energy spectra which allow to determine the origin of the background. However the background level achieved with the plastic scintillator veto is already low enough to perform a meaningful experiment.

\subsection{Random coincidences}

In each coincidence experiment the time random coincidence rate can have a serious impact on the accuracy of the measurement. In the context of the present experiment a random coincident event is (dominantly) two $662 \mathrm{keV}$ gamma-rays which are emitted from the source at similar points in time and deposit a part of their energy in two $\mathrm{LaBr}_{3}$-detectors such that the sum energy is in the energy window of $\sim 652$ to $\sim 672 \mathrm{keV}$.

A typically time spectrum of the two-photon decay experiment is shown on the left-hand side of Fig. 4.2. The so called true coincidence peak, which is due to two-photon and background events - sits on a flat distribution caused by random coincidences. In order to subtract the random coincidences one has to set a time gate of width $\tau$ on the true coincidence peak. For all events which fall in this region the sum energy spectrum is created which contains contributions from random coincidences as well as true coincidences. Next a time gate of width $\tau^{\prime}$ is set an the flat distribution of the random coincidences. The corresponding sum energy spectrum is 
scaled with a factor $\tau / \tau^{\prime}$ and then subtracted from the sum energy spectrum belonging to the time gate of width $\tau$. The resulting spectrum corresponds to the sum energy spectrum of the true coincidences. The uncertainty introduced by this procedure is determined by the ratio of the number of counts in the two-photon coincidence peak and the square root of the number of random coincidences in the time window $\tau$. The influence of the source strength and the efficiency of the $\mathrm{LaBr}_{3}$-detectors on this ratio are investigated in the following.

The rate of random coincidences in the time window $\tau$ is given through [Kno10]

$$
\begin{aligned}
R_{\text {rand }} & =\tau \cdot R_{1} \cdot R_{2} \cdot f \\
& =\tau \cdot \epsilon_{\text {tot }}^{2}(662 \mathrm{keV}) \cdot R_{1 \gamma}^{2} \cdot f,
\end{aligned}
$$

with $R_{1}$ and $R_{2}$ being the singles rate in each detector which are assumed to be the same, $\epsilon_{\text {tot }}$ is the total efficiency of a $\mathrm{LaBr}_{3}$-detector for an energy of $662 \mathrm{keV}$ and $R_{1 \gamma}$ is the number of $662 \mathrm{keV}$ photons emitted from the source per second. With $f=1$ the formula describes the total random rate. However, only the coincident events with a sum energy between $\sim 652$ and $\sim 672 \mathrm{keV}$ are of interest, i.e. the total random rate is reduces by a factor $f$ which is constant and can be either simulated or taken from experiment (typically $f \approx 13$ ).

The two-photon rate

$$
R_{2 \gamma}=2 \cdot \epsilon_{\text {full }}\left(E_{1}\right) \cdot \epsilon_{\text {full }}\left(E_{2}\right) \cdot R_{1 \gamma} \cdot\left(\Gamma_{\gamma \gamma} / \Gamma_{\gamma}\right),
$$

is determined by the full energy efficiencies at the energies $E_{1}$ and $E_{2}$ with $E_{1}+E_{2}=662 \mathrm{keV}$ and the rate of emitted two-photon events $R_{1 \gamma} \cdot \Gamma_{\gamma \gamma} / \Gamma_{\gamma}$. The ratio $\Gamma_{\gamma \gamma} / \Gamma_{\gamma}$ is the branching ratio of the two-photon decay. A measure for the uncertainty introduced by the subtraction of the random coincidences is given by the ratio

$$
\frac{\sqrt{R_{\text {rand }} \cdot \mathrm{t}}}{R_{2 \gamma} \cdot \mathrm{t}}=\frac{\sqrt{\tau} \cdot \epsilon_{\mathrm{tot}}(662 \mathrm{keV}) \cdot f}{2 \cdot \epsilon_{\text {full }}\left(E_{1}\right) \cdot \epsilon_{\text {full }}\left(E_{2}\right) \cdot\left(\Gamma_{\gamma \gamma} / \Gamma_{\gamma}\right)} \cdot \sqrt{\mathrm{t}},
$$

with $\mathrm{t}$ being the measurement time. Eq. 3.3 suggests that it is best to position the detectors close to the source because the two-photon rate scales with $1 / d^{4}$ ( $d$ is the distance between the source and the detectors) and to use the strongest available source in order to collect as many two-photon events as possible. However, the main contribution to the total uncertainty of the two-photon branching ratio is introduced by the subtraction of the random coincidences. Interestingly the source strengths cancel in the nominator and denominator, i.e. the source strength has a minor influence on the final uncertainty. Furthermore the ratio in Eq. 3.4 scales with $d^{2}$, i.e. positioning the detectors close to the source is not as important as suggested by Eq. 3.3. This result motivated the detector arrangement presented in Sect. 3.1, where the detectors were positioned at a distance of $d=22 \mathrm{~cm}$ in order to include the pairs with a relative angle of $144^{\circ}$. At smaller distances than $22 \mathrm{~cm}$, it is difficult to put a sufficient amount of lead between these detector pairs to block Compton scattering reliably.

\subsection{Data taking conditions and electronic setup}

The two-photon decay experiment was conducted at the institute of nuclear physics in Darmstadt in the end of 2013 and in the beginning of 2014. The total measurement time was 
52.74 days. Five $\mathrm{LaBr}_{3}$-detectors were used which had a cylindrical shape and a diameter of $7.62 \mathrm{~cm}$ and a length of $7.62 \mathrm{~cm}$. In total 10 detector pairs can be formed, five with a relative angle of $72^{\circ}$ and five with an angle of $144^{\circ}$. In the beginning of the experiment there was a problem with the lead shield between one of the $72^{\circ}$-pairs. Hence this pair was not considered in the analysis of the experiment. Each detector was positioned $22 \mathrm{~cm}$ away from the ${ }^{137} \mathrm{Cs}$ source.

\begin{tabular}{c|ccc}
\hline \hline angle group & no. of pairs & backg. (1/d) & m. time (d) \\
\hline $72^{\circ}$ & $5(4)$ & $4.3(5)$ & $52.74 \mathrm{~d}$ \\
$144^{\circ}$ & 5 & $0.7(2)$ & $52.74 \mathrm{~d}$ \\
\hline \hline
\end{tabular}

Table 3.1: Information on the two-photon experiment. Shown are the number of pairs per angle, the number of background events per day and pair and the total measurement time.

According to Eq. 3.4 the accuracy of the subtraction of the time random coincidences is independent of the source strength. Hence the ${ }^{137} \mathrm{Cs}$ source was chosen to be as strong as possible in order to collect a large number of two-photon events. However at the institute of nuclear physics only a few appropriate ${ }^{137} \mathrm{Cs}$ sources were available. It was decided to combine three source with a total activity of $606.11 \mathrm{kBq}\left(5^{\text {th }}\right.$ October 2013). The branching of the $662 \mathrm{keV}$ gamma-ray amounts to 0.805 . Therefore the total number of $662 \mathrm{keV}$ gamma-rays emitted per second is $487.92 \cdot 10^{3}$.

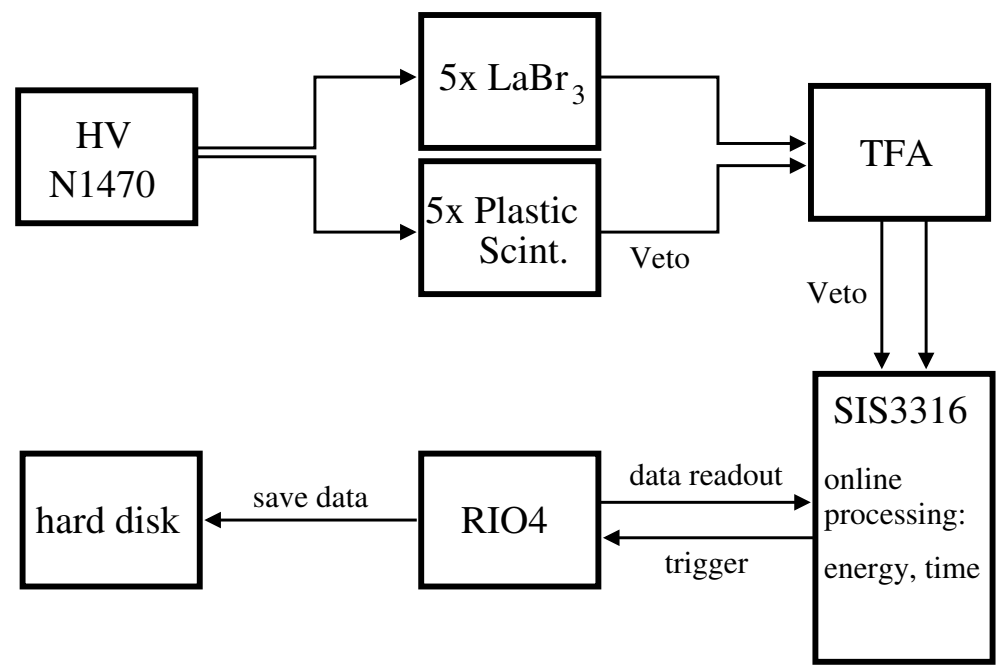

Figure 3.6: Electronic setup of the two-photon experiment. The ten signals of the $\mathrm{LaBr}_{3}$ detectors and plastics scintillators are fed in an in-house TFA to amplify the signals. They are then digitized by the SIS3316 module. The energy and point in time are determined by appropriate algorithms on the FPGA boards of the digitizer. The readout is controlled by the RIO4 module and the data is finally saved on hard disk.

The electronic setup of the experiment is presented in Fig. 3.6. The power supply of the five $\mathrm{LaBr}_{3}$-detectors and the five plastic scintillators were provided by three N1470 high-voltage modules from Caen company. The plastic scintillators are used to veto background events (see Sect. 3.2). The ten signals were amplified and slightly shaped through an in-house time-filter 
amplifier (TFA). Afterward they were fed into the SIS3316 module of Struck company. This digitizer has a sampling rate of $250 \mathrm{MHz}$ and a resolution of 14 bit. The data is processed online on the FPGA boards of the digitizer and the energy and time information are extracted. In order to obtain the time information a digital Constant Fraction Discriminator is applied to the recorded trace. A filter - which is similar to a digital QDC - is used to extract the energy information.

Whenever the digitizer registered an event, a trigger signal is sent to the RIO4 module, which starts the readout of the data via the VME backplane. The data is sent via an Ethernet connection and eventually saved on hard disk. The dead time of the data acquisition system was less than one percent for a total rate of $\sim 23 \mathrm{kHz}$. This is due to the two memory buffers of the digitizer, which allows to readout one of them while - during the readout process - the data is written to the other one. 


\section{Data analysis and results of the two-photon decay experiment}

This chapter gives detailed information on the analysis of the two-photon decay experiment. The raw data is saved on hard disk in list mode files. The relevant information, like time and energy of the corresponding events, are extracted using the data unpacker ucesb [Joh10] and is written in a 'root-tree' of the data analysis framework root [ROT]. This software package was used to further process and analyze the data.

\subsection{Energy and efficiency calibration}

As a first analysis step the energy spectra of the five $\mathrm{LaBr}_{3}$-detectors were calibrated using known gamma-ray transitions. The gains of the detectors depend on the room temperature which is not stable over the measurement time of $\sim 52$ days. On the left-hand side of Fig. 4.1 the energy shift $\Delta E$ of the $662 \mathrm{keV}$ transition is shown in dependence of the measurement time. In order to obtain an optimal energy resolution it is necessary to calibrate the detectors several times per day. In the present experiment this was done with a two point calibration using the $662 \mathrm{keV}$ transition and an $X$-ray with an energy of $73 \mathrm{keV}$. The dependence of the integral of the detector signal on the gamma-ray energy is assumed to be linear. This method allows to use the production runs of the experiment for the energy calibration. The energy calibration was repeated every $\sim 40 \mathrm{~min}$, which is sufficient to obtain a good energy resolution of $2.9-3.1 \%$ (FWHM) at $662 \mathrm{keV}$.

In order to determine the two-photon decay branching ratio it is necessary to know the absolute full energy efficiencies of the five detectors. This efficiency was measured at an energy of $662 \mathrm{keV}$ using one of the production runs. On the right-hand side of Fig. 4.1 the average efficiency of one detector (green data point) is compared to the result of a GEANT4 simulation (red data points). The simulation is approximately $10 \%$ higher than the experimental value. This deviation is due to the imprecise positioning of some lead bricks, which slightly block a part of the solid angle of each detector.

The coincident efficiency, i.e. the efficiency that both photons stemming from the two-photon decay are detected with the setup, can be derived on the following way. The single efficiencies are given by

$$
\begin{aligned}
\epsilon\left(E_{1}\right) & =\epsilon_{A}\left(E_{1}\right)+\epsilon_{B}\left(E_{1}\right) \\
\epsilon\left(662-E_{1}\right) & =\epsilon_{A}\left(662-E_{1}\right)+\epsilon_{B}\left(662-E_{1}\right) .
\end{aligned}
$$

with $\epsilon_{A(B)}$ being the full energy efficiency of detector $A(B)$. Their product gives the coincident efficiency

$$
\begin{aligned}
\epsilon_{\text {coinc }}\left(E_{1}\right) & =\epsilon_{A}\left(E_{1}\right) \cdot \epsilon_{A}\left(662-E_{1}\right)+\epsilon_{B}\left(E_{1}\right) \cdot \epsilon_{B}\left(662-E_{1}\right)+ \\
& \epsilon_{A}\left(E_{1}\right) \cdot \epsilon_{B}\left(662-E_{1}\right)+\epsilon_{A}\left(662-E_{1}\right) \cdot \epsilon_{B}\left(E_{1}\right) \\
& =\epsilon_{A}\left(E_{1}\right) \cdot \epsilon_{B}\left(662-E_{1}\right)+\epsilon_{A}\left(662-E_{1}\right) \cdot \epsilon_{B}\left(E_{1}\right)
\end{aligned}
$$



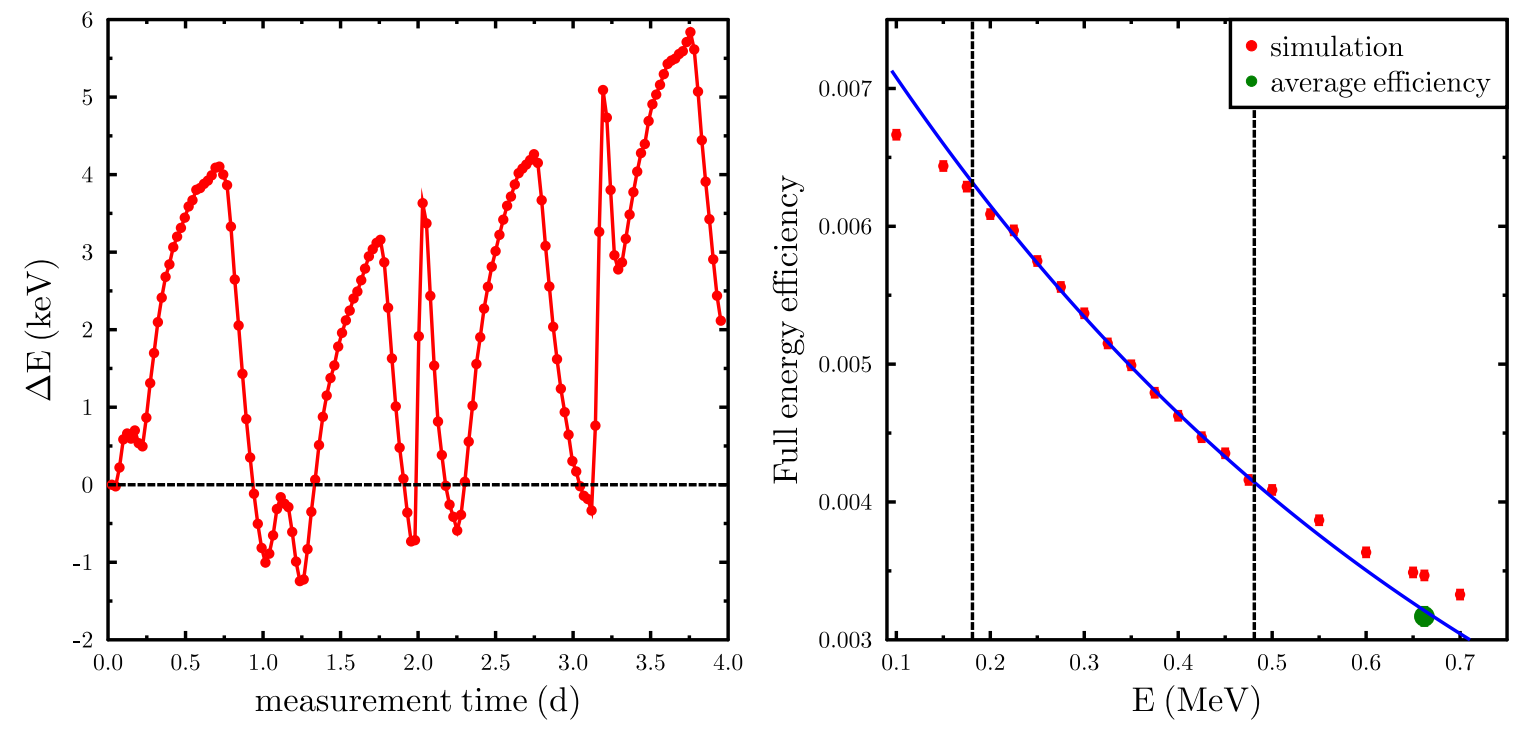

Figure 4.1: Long-term stability of the energy calibration, time dependence of the peak position of the $662 \mathrm{keV}$ transition over 4 days (left-hand side). Full energy efficiency of one $\mathrm{LaBr}_{3}$-detector obtained from a GEANT4 simulation is shown on the right-hand side (red data points). In the relevant energy range - marked with the dashed black lines - the full energy efficiency can be approximated with an exponential function (solid blue line). The measured average efficiency of one detector at $662 \mathrm{keV}$ is shown with the green data point. The measured data point is lower than the results of the simulation because of lead bricks which block a part of the solid angle of each detector.

The first and second term in Eq. 4.2 do not contribute, since they consider the cases where both photons hit the same detector which would not be detected as two separate events. In principle $\epsilon_{\text {coinc }}\left(E_{1}\right)$ depends on the energy sharing of both emitted photons.

In the present experiment however, the only considered events were those where the energies of the coincident gamma-rays $E_{1}$ and $E_{2}$ fulfilled the condition $\left|E_{1}-E_{2}\right|<300 \mathrm{keV}$ (see Sect. 4.4). This energy region is marked in Fig. 4.1 with dashed lines. Within this region, the simulated efficiency can be described to a good approximation assuming $\epsilon(E)=a \cdot \exp (-b E)$. Therefore the products $\epsilon_{A} \cdot \epsilon_{B}$ in Eq. 4.2 are independent of the energy $E_{1}$. The coefficients $a$ and $b$ were determined with a least squares fit to the simulated efficiency and amount to $a=0.0081$ and $b=1.41$. The simulated data points where scaled to the measured efficiency at $662 \mathrm{keV}$ for each detector and the coefficient $a$ was corrected appropriately. The final coincident efficiency for the detector pair $i$ consisting of detectors $A$ and $B$ is given through ( $b$ is the same for all detector pairs)

$$
\epsilon_{\text {coinc }}^{i}=2 a_{A} a_{B} \cdot \exp (-b \cdot 0.662)
$$

\subsection{Subtraction of the time random coincidences}

The time random coincidences were subtracted following the method outlined in Sect. 3.3. The five $\mathrm{LaBr}_{3}$-detectors were time aligned using the information obtained from a ${ }^{60} \mathrm{Co}$ measurement. The time spectrum for an angle of $144^{\circ}$ is displayed on the left-hand of Fig. 4.2. For this 
spectrum (and all spectra which follow) it was required that the energy in each detector is larger than $100 \mathrm{keV}$. Otherwise the background would increase drastically. A time gate with a width of $\tau=2.4 \mathrm{~ns}$ was used to select the true coincidences. The width was chosen to be as small as possible in order to suppress 'sequential' Compton scattering (see Sect. 3.1), and to improve the accuracy of the subtraction of the random coincidences (see Sect. 3.3). In order to subtract the random coincidences two time gates with a width of $\tau^{\prime}=56$ ns were set on the left-hand and the right-hand side of the true coincidence peak.

The corresponding sum energy spectra of all events fulfilling the time conditions imposed by the random coincidences time gates (solid red curve) and true coincidences time gate (solid blue curve) are shown in Fig. 4.3 for both angle groups. The sum energy spectrum of the random coincidences were scaled with a factor $\tau /\left(2 \tau^{\prime}\right)$ to correct for the different gate lengths. The final sum energy spectrum of the true coincidences is obtained by subtracting the red curve from the blue curve.
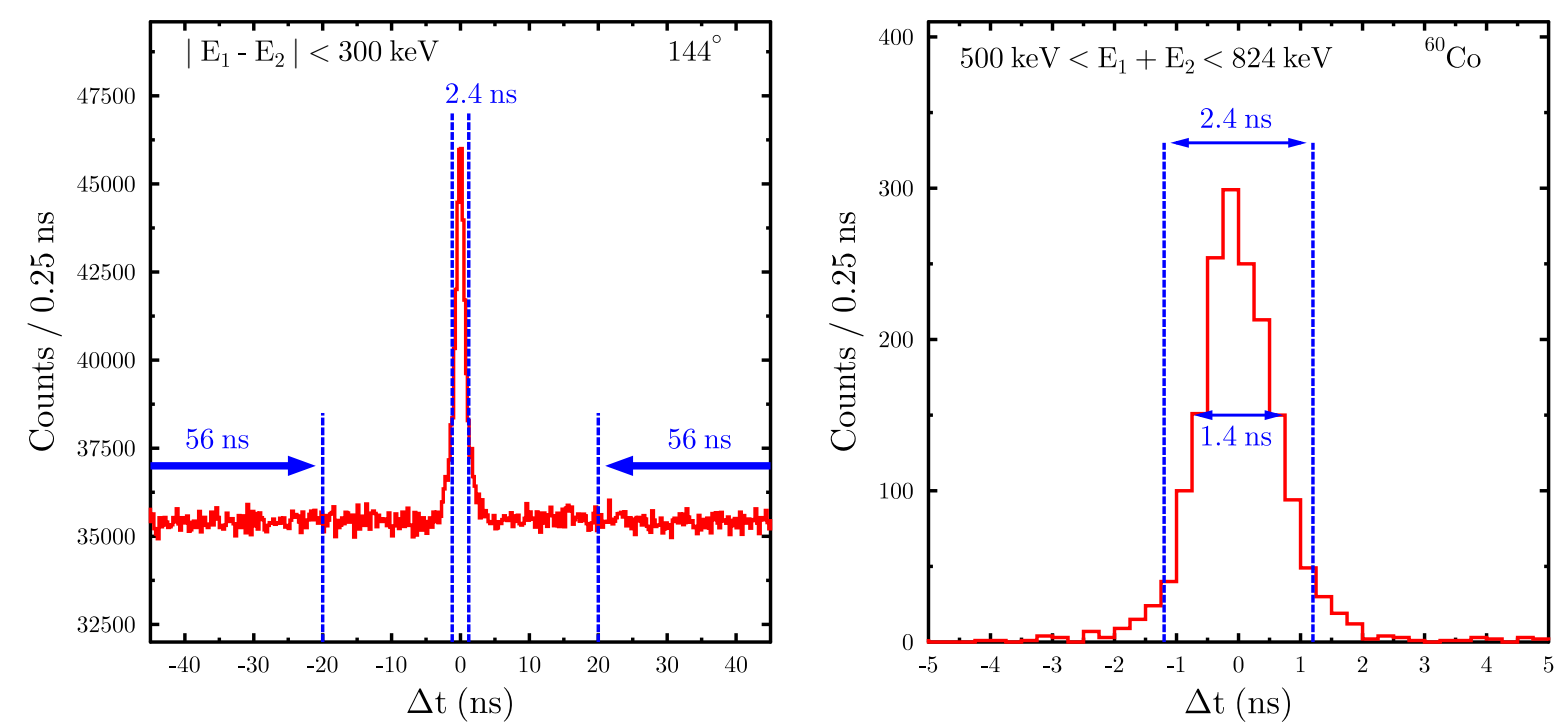

Figure 4.2: Time spectrum of the two-photon decay experiment of all detector pairs with an relative angle of $144^{\circ}$ (left-hand side). The time gate on the true coincidences has a width of $2.4 \mathrm{~ns}$. In order to subtract the random coincidences large time gates on the left- and right-hand side of the true coincidences peak were chosen with a width of 56 ns. The five $\mathrm{LaBr}_{3}$-detectors were time aligned with a ${ }^{60} \mathrm{Co}$ measurement (right-hand side). The achieved time resolution amounts to $\sim 1.4 \mathrm{~ns}$ (FWHM).

The peak at $1324 \mathrm{keV}$ is due to two $662 \mathrm{keV}$ gamma-rays which deposited their full energy in two detectors. The blue and the red curves (almost) match for this energy. This is expected since such events are clearly time random coincidences. At energies $<600 \mathrm{keV}$ one can see for both angles an accumulation of true coincidences which are discussed in the next section.

The width $\tau$ of the gate on the true coincidences gate is not large enough to collect all twophoton events. In order to determine the fraction of events outside of this gate a ${ }^{60}$ Co source measurement was conducted. The resulting time coincidence peak with a full width at half max of $\sim 1.4 \mathrm{~ns}$ is presented on the right-hand side of Fig. 4.2. The energy condition $500 \mathrm{keV}<$ $E_{1}+E_{2}<824 \mathrm{keV}$ is centered around $662 \mathrm{keV}$ to provide similar energy conditions as for the actual two-photon decay experiment. In principle it is desirable to use identical gates e.g. $\mid E_{1}-$ 
$E_{2} \mid<300 \mathrm{keV}$ and $652 \mathrm{keV}<\left|E_{1}+E_{2}\right|<672 \mathrm{keV}$ as in Fig. 4.5. However, the statistics of the ${ }^{60} \mathrm{Co}$ measurements were not sufficient to apply such strict conditions. Nevertheless, the time resolution is expected to change only slightly between the conditions applied in Fig 4.2 and the one in the actual experiment. The number of events outside the gate on the true coincidence peak were measured to be $\sim 10 \%$ of the total number of events. It is not possible to obtain this number directly from the production runs due to the contributions of background events (see Sect. 3.2) to the true coincidence peak which exhibit a broader time peak.
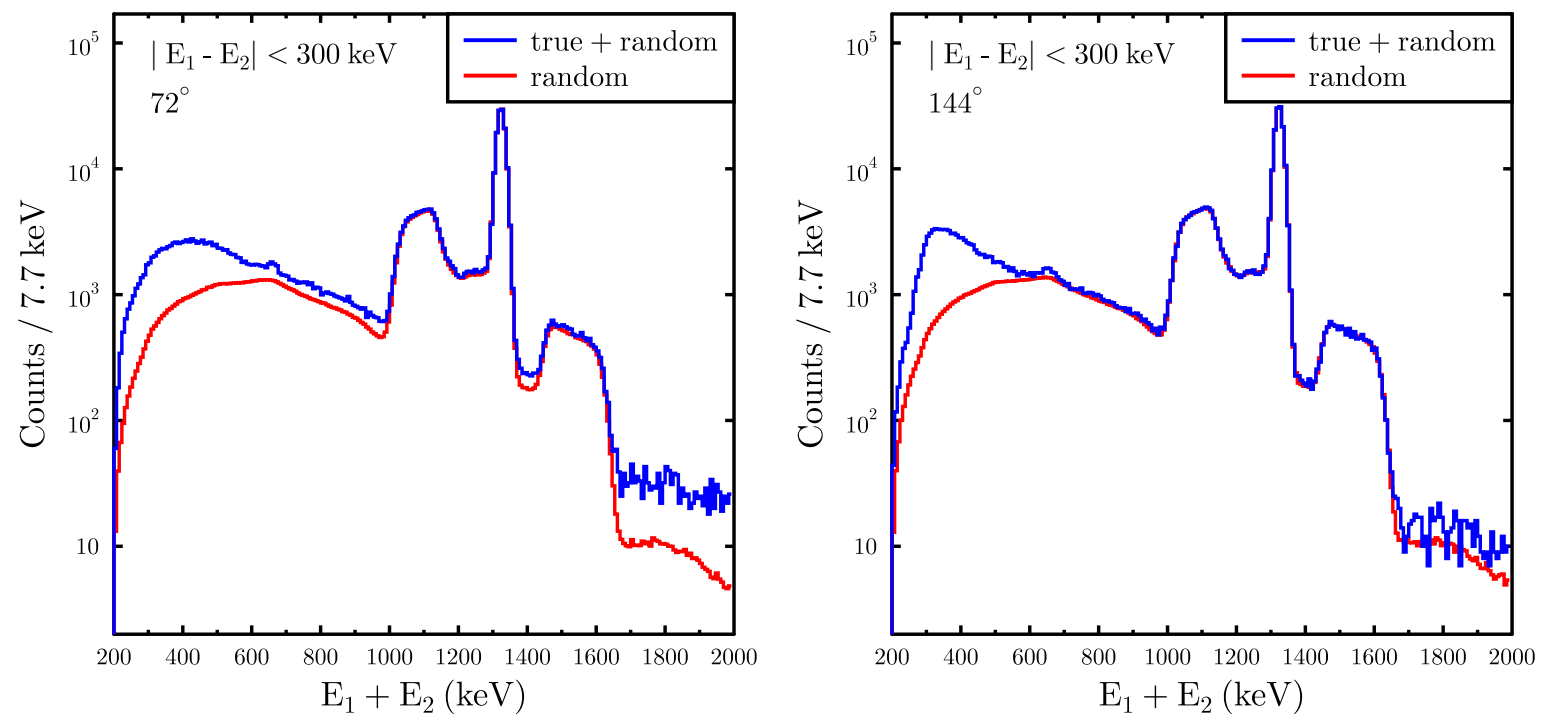

Figure 4.3: Sum energy spectra of all events satisfying the random (red) and true (blue) coincidence time conditions shown in Fig. 4.2 for both detector angles. The random coincidences are scaled with an appropriate factor correcting for the different gate widths.

\subsection{Background conditions of the two-photon decay experiment}

The problem of the background being present even without a radioactive was discussed in Sect. 3.2. The background for an angle of $144^{\circ}$ is a factor of $\sim 6$ smaller than the background at an angle of $72^{\circ}$ because of the larger distance between both detectors. In Fig. 4.4 the sum energy spectrum of a 3.84 days long measurement without a radioactive source (blue data points) is compared to a 13.33 days long measurement with a ${ }^{137} \mathrm{Cs}$ source (red data points). In both spectra the random coincidences are already subtracted. In order to allow a meaningful comparison the background measurement is scaled appropriately correcting for the different measurement times. Clearly, for energies $>600 \mathrm{keV}$ both spectra almost match i.e. the deviations above this energy seen in Fig. 4.3 between the blue and red curves are due to the background that is present without a radioactive source. Particularly the background under the $662 \mathrm{keV}$ of the two-photon decay is determined by this type of background.

At energies $<600 \mathrm{keV}$ the background related to the radioactive source starts to dominate the sum energy spectra. Presumably the $662 \mathrm{keV}$ gamma-ray stemming from the one-photon decay hits one detector and is than scattered on the surrounding material through multiple Compton scattering processes in a second detector. A Monte-Carlo simulation of the entire 
setup could help to understand this huge accumulation of counts at lower energies. However it is well separated in energy from the two-photon peak at $662 \mathrm{keV}$ and does not prevent the determination of the two-photon decay branching ratio.

In general the background in the region of the two-photon peak at $662 \mathrm{keV}$ is smooth in energy and can be well described assuming an exponential function (see Sect. 4.4).
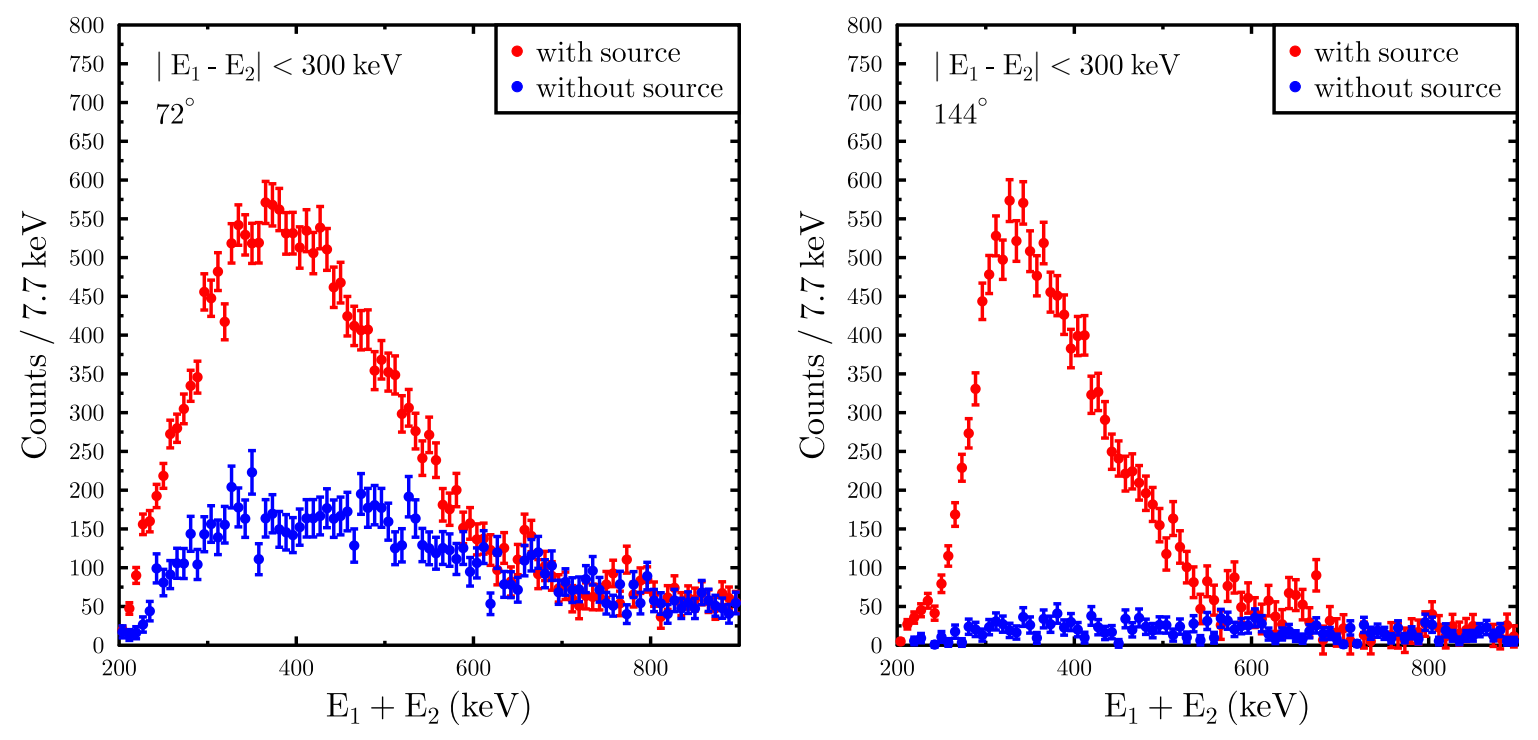

Figure 4.4: Sum energy spectra of the measurements with a ${ }^{137} \mathrm{Cs}$ source (red) and without radioactive source (blue) for both detector angles. At sum energies $<600 \mathrm{keV}$ the contribution from the source dominates, while at higher energies the contributions from not source related background becomes important. In particular the background under the $662 \mathrm{keV}$ peak is mainly determined through this background.

\subsection{Two-photon decay branching ratio}

On the left-hand side of Fig. 4.5 the region of interest around $662 \mathrm{keV}$ of Fig. 4.3 is shown. Even without the subtraction of the time random coincidences (blue data points) one can clearly identify a peak at the expected energy (red data points). The error bars of the sum energy spectrum of the time random coincidences are negligible due to the large time gates with a total length of 112 ns.

On the right-hand side of Fig. 4.5 is shown the sum energy spectrum for an angle of $72^{\circ}$ after subtraction of the time random coincidences. The background can be well described assuming a functional dependence of $B k g(x)=\exp \left(a+b(x-640)+c(x-640)^{2}\right)$, with $x$ being the sum energy in $\mathrm{keV}$. The expected 'sequential' Compton scattering peak is not present in the spectrum due to the chosen energy gate of $\left|E_{1}-E_{2}\right|<300 \mathrm{keV}$ which excludes the energies of $\sim 125 \mathrm{keV}$ and $\sim 478 \mathrm{keV}$. This energy gate was also chosen to suppress the background which is present without a radioactive source. A widening of this energy gate increases the contribution of this background significantly and makes an analysis of the two-photon peak difficult.

The peak at $662 \mathrm{keV}$ is fitted assuming a Gaussian peak shape. The width was kept constant at a value of $20.2 \mathrm{keV}$ which is the average energy resolution of the five $\mathrm{LaBr}_{3}$-detectors at $662 \mathrm{keV}$ determined from the singles spectra. In order to justify this choice the energy dependence of 

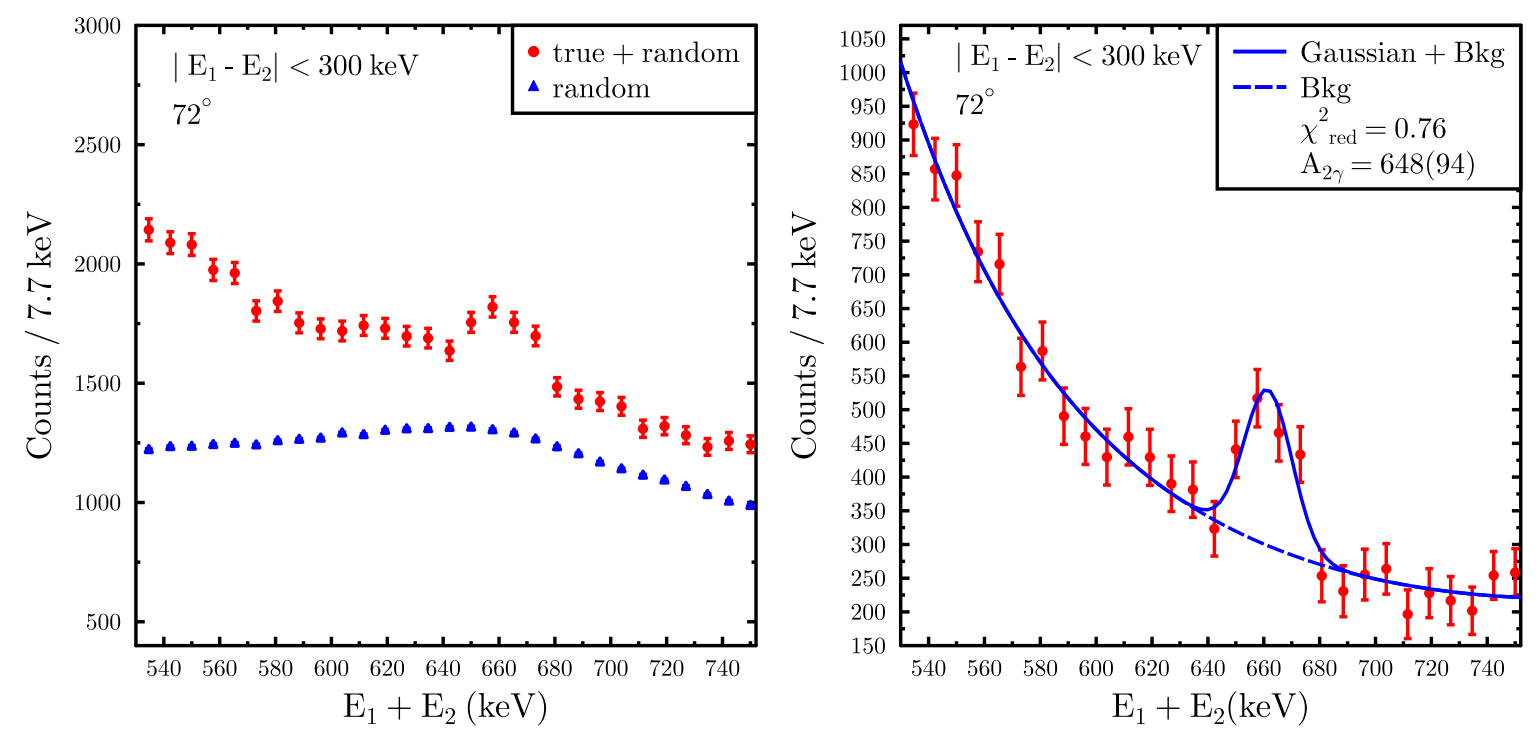

Figure 4.5: The figure on the left-hand side is the same like the figures shown in Fig. 4.3 but focused on the region around $662 \mathrm{keV}$. The $662 \mathrm{keV}$ peak due to the two-photon decay is clearly visible in the not random subtracted spectrum. The random subtracted spectrum is shown on the right-hand side. The background is fitted with an exponential function and the peak with a Gaussian function with a fixed width known from previous measurements.

the energy resolution of each detector was determined with various radioactive sources. The resulting functions were used as an input for the following simulation. The energy sharing of the two photons stemming from the two-photon decay is assumed to follow one of the distributions given in Eqs. 2.12 and 2.13, i.e. either quadrupole-quadrupole or dipole-octupole. Large sets of two-photon events following these energy sharing distributions were created. The energies measured by the detectors were determined from Gaussian functions with centroids at the two two-photons energies and with widths taken from the measured energy resolution functions. The peak in the final sum energy spectra had a Gaussian shape with a width similar to the one measured in the singles spectra at $662 \mathrm{keV}$. This holds for both energy sharing distributions.

The centroid of the Gaussian function and its amplitude were free parameters in the fit. The reduced chi-square is excellent and amounts to $\chi_{\text {red }}^{2}=0.76$. The measured energy is at the expected value of $661.6(17) \mathrm{keV}$ and the peak area is determined to be $N_{\gamma \gamma}=648$ (94) counts for a total measurement time of 52.74 days.

The two-photon decay branching ratio can be calculated with the equation

$$
W^{\prime}(\theta) \cdot \frac{\Gamma_{\gamma \gamma}}{\Gamma_{\gamma}}=\frac{N_{\gamma \gamma} \cdot f \cdot \sum_{i=1}^{5} \epsilon_{i}(662 \mathrm{keV})}{\left(\sum_{i=1}^{5} N_{\gamma}^{i}\right) \cdot\left(\sum_{i=1}^{5} \epsilon_{\text {coinc }}^{i}\right)},
$$

with $\sum_{i=1}^{5} \epsilon_{i}(662 \mathrm{keV})$ being the sum over the full energy efficiencies at $662 \mathrm{keV}$ for the five detectors. $W^{\prime}(\theta)$ is the angular correlation function integrated over the solid angle of each detector, $N_{\gamma}^{i}$ is the total number of counts for the peak at $662 \mathrm{keV}$ in the singles spectra of detector $i . \epsilon_{\text {coinc }}^{i}$ is the energy-independent coincident efficiency of detector pair $i$ defined in Eq. 4.3. During the measurement it was necessary to remove the ${ }^{137} \mathrm{Cs}$ source several times. 
Since it was impossible to position the source at identical locations the $\epsilon_{\text {coinc }}^{i}$ and $\epsilon_{i}(662 \mathrm{keV})$ efficiencies change slightly within a few percent. The efficiencies were averaged, weighted with the corresponding measurement times.

The factor $f=f_{t}+f_{e}$ has two contributions. The term $f_{t}$ corrects $N_{\gamma \gamma}$ for the number of events that are outside the time gate of $2.4 \mathrm{~ns}$ and amounts to 1.119(6). As described in Sect. 4.2 this value was determined with a ${ }^{60} \mathrm{Co}$ source measurement. Due to the energy condition of $\left|E_{1}+E_{2}\right|<300 \mathrm{keV}$ the photon energies from $0-181 \mathrm{keV}$ and $481-662 \mathrm{keV}$ are not included in the peak area $N_{\gamma \gamma}$ of Fig. 4.5. Their contributions were included with the term $f_{e}$ and were estimated assuming a pure quadrupole-quadrupole $\left(f_{e}=1.12\right)$ and a pure dipole-octupole energy sharing distributions $\left(f_{e}=1.54\right)$.

The sum over the averaged absolute full energy efficiencies is $0.0158(3)$. Moreover the sum over the coincident efficiencies $\epsilon_{\text {coinc }}^{i}$ of all detector pairs with a relative angle of $72^{\circ}$ amounts to $1.05(4) \cdot 10^{-4}$. The uncertainty of the efficiency of a single detector is assumed to be $2 \%$ and due to the imprecisely known distance from source to detector and uncertainties of the source strengths. The total number of counts in the $662 \mathrm{keV}$ peaks is $3.507 \cdot 10^{10}$ with a negligible statistical uncertainty. The uncertainty of the peak area $N_{\gamma \gamma}$ is taken from the fit results and includes correlated errors with the background function.

With these values in hand one can calculate the two-photon decay branching ratio at an angle of $72^{\circ}$ using Eq. 4.4. With $f_{e}=1$ the branching ratio - multiplied with the angular correlation function $-W^{\prime}\left(72^{\circ}\right) \cdot \Gamma_{\gamma \gamma} / \Gamma_{\gamma}$ amounts to $1.56(23) \cdot 10^{-6}$. The final uncertainty is dominated by the uncertainty of the peak area. For the assumption of a pure quadrupolequadrupole transition $\left.W^{\prime}\left(72^{\circ}\right) \cdot \Gamma_{\gamma \gamma} / \Gamma_{\gamma}\right)^{\mathrm{qq}}=1.75(27) \cdot 10^{-6}$ and for a pure dipole-octupole transition $\left.W^{\prime}\left(72^{\circ}\right) \cdot \Gamma_{\gamma \gamma} / \Gamma_{\gamma}\right)^{\text {do }}=2.40(36) \cdot 10^{-6}$. The results are summarized in Tab. 4.1.

\begin{tabular}{c|c|c}
\hline \hline$\theta$ & $72^{\circ}$ & $144^{\circ}$ \\
\hline $\mathrm{E}_{\gamma \gamma}$ & $661.6(17)$ & $664.2(28)$ \\
$W^{\prime}(\theta) \cdot \Gamma_{\gamma \gamma} / \Gamma_{\gamma}$ & $1.56(23) \cdot 10^{-6}$ & $0.70(18) \cdot 10^{-6}$ \\
$W^{\prime}(\theta) \cdot\left(\Gamma_{\gamma \gamma} / \Gamma_{\gamma}\right)^{\mathrm{qq}}$ & $1.75(27) \cdot 10^{-6}$ & $0.85(22) \cdot 10^{-6}$ \\
$W^{\prime}(\theta) \cdot\left(\Gamma_{\gamma \gamma} / \Gamma_{\gamma}\right)^{\mathrm{do}}$ & $2.40(36) \cdot 10^{-6}$ & $1.33(35) \cdot 10^{-6}$ \\
\hline \hline
\end{tabular}

Table 4.1: Summary of the results of the two-photon decay experiment. The branching ratios for an angle of $72^{\circ}$ and $144^{\circ}$ were determined with different energy gates, $\left|E_{1}-E_{2}\right|<300 \mathrm{keV}$ and $\left|E_{1}-E_{2}\right|<250 \mathrm{keV}$ respectively. The contributions from the parts outside of these energy regions are included assuming a pure quadrupolequadrupole transition $\left(\Gamma_{\gamma \gamma} / \Gamma_{\gamma}\right)_{\text {corr }}^{\mathrm{qq}}$ and a pure octupole-dipole transition $\left(\Gamma_{\gamma \gamma} / \Gamma_{\gamma}\right)_{\text {corr }}^{\text {do }}$.

For an relative detector angle of $144^{\circ}$ the analysis steps are identical to the one outlined for an angle of $72^{\circ}$. However one has to take into account the different energy of the 'sequential' Compton scattering peak at $\sim 650 \mathrm{keV}$. In the following the process of 'sequential' Compton scattering is discussed in more detail at the example of the $144^{\circ}$-data. The two photons contributing to this process have energies of $\sim 478 \mathrm{keV}$ and $\sim 172 \mathrm{keV}$. On the left-hand side of Fig. 4.6, the sum energy spectrum is shown after subtraction of the time random coincidences (red data points). The energy condition requiring $\left|E_{1}-E_{2}\right|<350 \mathrm{keV}$ still includes the expected photon energies of 'sequential' Compton scattering. Clearly, a strong peak is visible at the expected energy of $\sim 650 \mathrm{keV}$. In order to confirm its origin two additional energy gates were set from $462 \mathrm{keV}$ to $493 \mathrm{keV}$ and from $157 \mathrm{keV}$ to $187 \mathrm{keV}$. Events with sum energies within these two 
gates were not considered. The resulting spectrum is also shown for comparison in Fig. 4.6. Clearly, the peak is strongly suppressed proving that its origin is due to photons with energies expected for 'sequential' Compton scattering.

Furthermore an energy gate was set on the peak at $\sim 650 \mathrm{keV}$ requiring that $630 \mathrm{keV}<E_{1}+$ $E_{2}<680 \mathrm{keV}$. The corresponding time spectrum is shown on the right-hand side of Fig. 4.6. Two time structures can be identified on the right-hand and left-hand sides of $\Delta t=0$. Since all detectors are $22 \mathrm{~cm}$ away from the ${ }^{137} \mathrm{Cs}$ source, a gamma-ray exhibiting 'sequential' Compton scattering has to travel additional $44 \mathrm{~cm}$ which corresponds to a time difference of $\sim 1.5 \mathrm{~ns}$. Indeed, the centroids of both peaks are at $\sim \pm 1.5 \mathrm{~ns}$. The occurrence of two time structures are due to the possibility that the $662 \mathrm{keV}$ gamma-ray interacts first with detector 1 and is then scattered into detector 2 or that this gamma-ray interact first with detector 2 and then with detector 1 . The investigations of the time and sum energy spectra clearly show that the peak seen at $\sim 650 \mathrm{keV}$ is indeed due to the 'sequential' Compton scattering process outlined in Sect. 3.1.
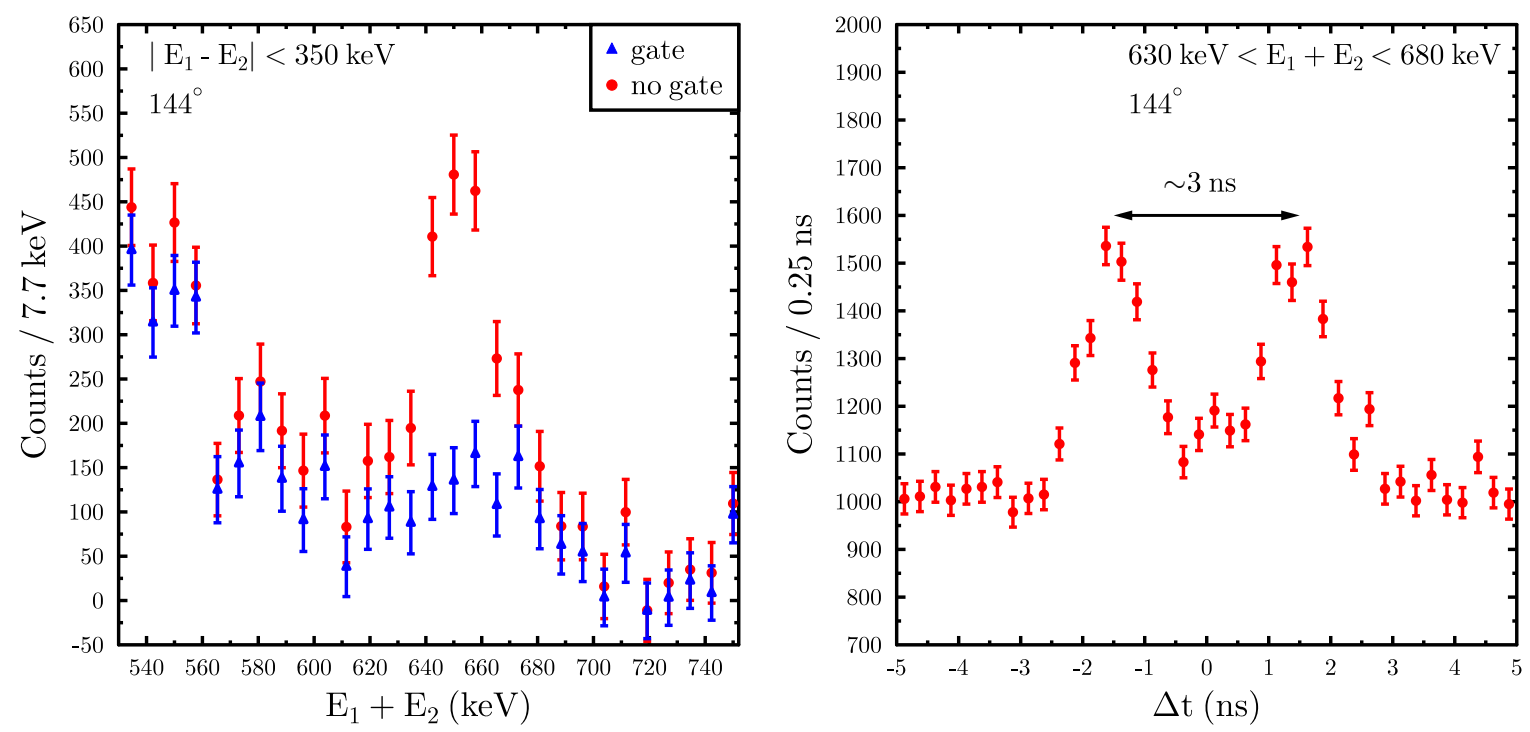

Figure 4.6: Illustrated is the impact of the 'sequential' Compton scattering process described in Sect. 3.1 for an angle of $144^{\circ}$. On the left-hand side the peak in the sum energy spectrum (red) is at the expected energy of $\sim 650 \mathrm{keV}$ for the 'sequential' Compton scattering process. This effect can be suppressed with appropriate veto-gates on the expected energies ( $\sim 78 \mathrm{keV}$ and $\sim 172 \mathrm{keV}$ ) in each detector (blue). The time spectrum on the left-hand side is created with an energy gate on the 'sequential' Compton scattering peak ( $630 \mathrm{keV}<E_{1}+E_{2}<680 \mathrm{keV}$ ). The two time peaks on the left-hand side and the on the right-hand side of $\Delta t=0$ are due to the 'sequential' Compton scattering process. The two peaks develop because the $662 \mathrm{keV}$ photon can interact first with detector 1 and than with detector 2 or vice versa. Since the path length between both detectors is $\sim 44 \mathrm{~cm}$, one expects both peaks to be separated by $\sim 3 \mathrm{~ns}$. That is the case.

In order to measure the two-photon decay probability at an angle of $144^{\circ}$, it is necessary to chose an energy gate that suppresses 'sequential' Compton scattering reliably. The energy gate $\left|E_{1}-E_{2}\right|<300 \mathrm{keV}$ is not sufficient, since it allows energies in the range $181 \mathrm{keV}$ to $481 \mathrm{keV}$, i.e. 
very close to $\sim 172 \mathrm{keV}$ (for $E_{1}+E_{2}=662 \mathrm{keV}$ ). Therefore the energy condition was reduced to $\left|E_{1}-E_{2}\right|<250 \mathrm{keV}$, which limits the accepted energies from $206 \mathrm{keV}$ to $456 \mathrm{keV}$.

The resulting sum energy spectrum is shown on the left-hand side of Fig. 4.7. Again one can clearly see a peak at $662 \mathrm{keV}$. The background and the peak were fitted using the same functions and conditions (i.e. fixed width of the Gaussian function) as for $72^{\circ}$. Surprisingly the peak area $N_{\gamma \gamma}=307(78)$ is more than a factor of two smaller than for $72^{\circ}$. Later it will be shown that this difference is not due to the different energy conditions on $\left|E_{1}-E_{2}\right|$.

The two-photon decay branching ratio is again calculated with Eq. 4.4. The sums over $\epsilon_{i}, N_{\gamma}^{i}$ and the correction factor $f_{t}$ are the same as for $72^{\circ}$. The sum over the coincident efficiencies $\epsilon_{\text {coinc }}^{i}$ is slightly different and amounts to $1.09(4) \cdot 10^{-4}$, since different detector pairs are involved. The two-photon branching ratio at an angle of $144^{\circ}$ is determined to be $W^{\prime}\left(144^{\circ}\right) \cdot \Gamma_{\gamma \gamma} / \Gamma_{\gamma}=0.70(18)$. The correction factors $f_{e}$ are different due to the different energy condition on $\left|E_{1}-E_{2}\right|$. For a pure quadrupole-quadrupole transition $W^{\prime}\left(144^{\circ}\right) \cdot\left(\Gamma_{\gamma \gamma} / \Gamma_{\gamma}\right)^{\mathrm{qq}}$ amounts to $0.85(22)$ and for a pure octupole-dipole transition $W^{\prime}\left(144^{\circ}\right) \cdot\left(\Gamma_{\gamma \gamma} / \Gamma_{\gamma}\right)^{\text {od }}=1.33(35)$.
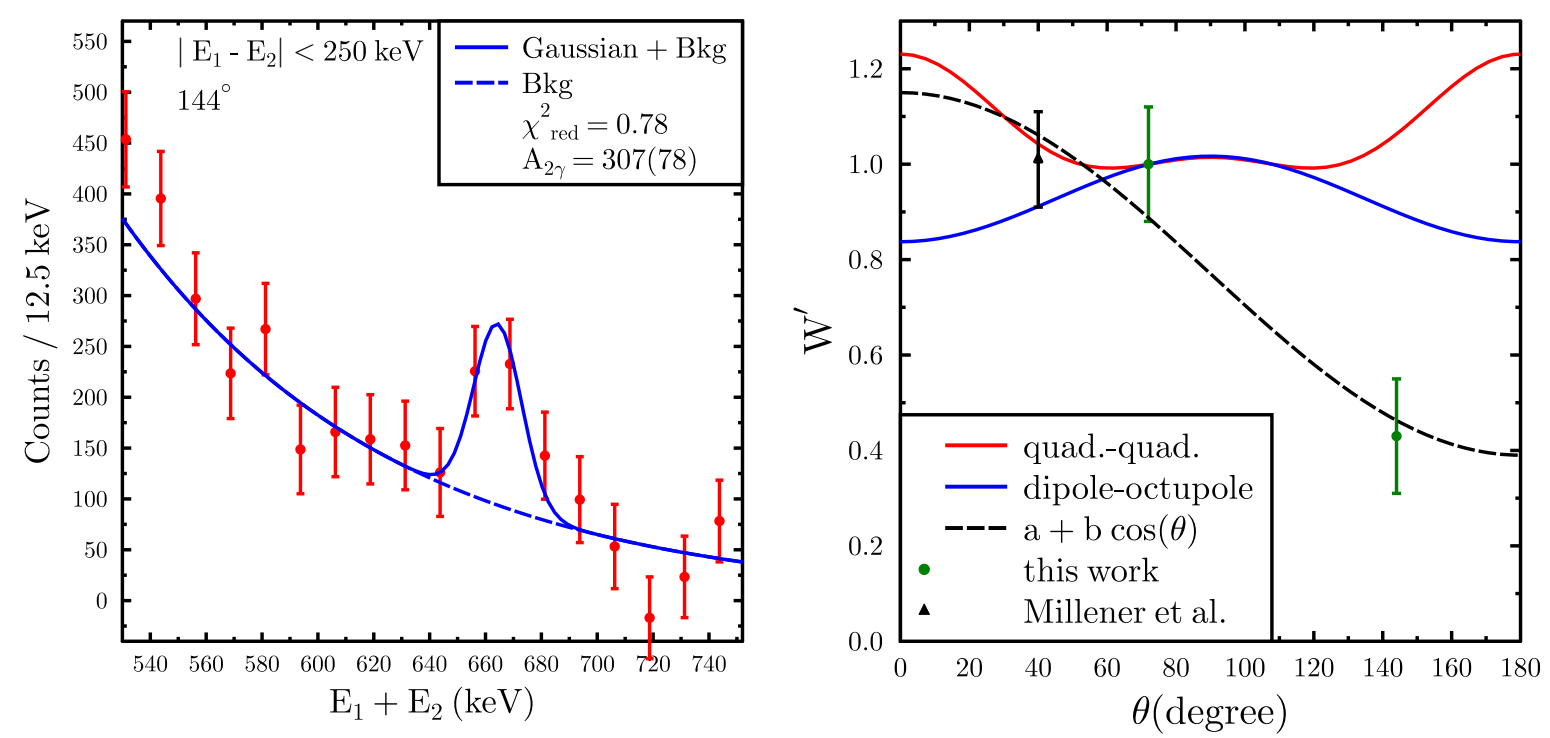

Figure 4.7: Random subtracted sum energy spectrum for an angle of $144^{\circ}$ (left-hand side). The energy gate on $\left|E_{1}-E_{2}\right|$ is limited to $250 \mathrm{keV}$ to suppress the 'sequential' Compton scattering process. On the right-hand side the angular distribution $W^{\prime}(\theta)$ of the twophoton decay branching ratio is shown. Clear deviations between theory - assuming pure quadrupole-quadrupole or dipole-octupole transitions - and experiment can be found. The experimental data are normalized to the data point measured at an angle of $72^{\circ}$. The data point at $\sim 40^{\circ}$ is taken from Ref. [Mil14].

The angular distribution of the two-photon decay probability is shown on the right-hand side of Fig. 4.7. The experimental data points are compared to the theoretical angular distributions for a pure quadrupole-quadrupole (solid red line) and a pure dipole-octupole transitions (solid blue line). The experimental data, as well as the theoretical angular distributions, are normalized to the data point at $72^{\circ}$. For this data point the energy condition on $\left|E_{1}-E_{2}\right|<X$ was changed from $X=300 \mathrm{keV}$ to $X=250 \mathrm{keV}$ for the $72^{\circ}$-data to allow a direct comparison to the 
data point at $144^{\circ}$. This also proves that the difference between $\Gamma_{\gamma \gamma} / \Gamma_{\gamma}$ at $72^{\circ}$ and $144^{\circ}$ in Tab. 4.1 was not due the different energy conditions.

D.J. Millener and collaborators have also successfully measured the two-photon decay at an angle of the $\sim 40^{\circ}$ [Mil11, Mil14] with a setup consisting of two NaI detectors and a very long measurement time of several hundred days. Their data point - again requiring the energy condition $\left|E_{1}-E_{2}\right|<250 \mathrm{keV}$ - is also presented in Fig. 4.7. The excellent agreement between this data point and the data point at $72^{\circ}$ of the present work - measured with different experimental setups and analyzed by different people - gives confidence in the obtained results. However the data point at $144^{\circ}$ is off by more than a factor two from the expected angular distributions for pure multipole transitions. It should be noted that this data point was measured with the same five detectors as the data point at $72^{\circ}$. The detectors were simply combined differently to form five detector pairs with a relative angle of $144^{\circ}$. Hence it is difficult to attribute the strong deviations to an experimental error.

At the moment an explanation for the angular distribution is missing. One reason could be, that the model presented in Sect. 2.2 is too simple. Other multipole transitions with different angular distributions might be important. Furthermore it is possible that two multipole pairs contribute, which have the same multipoles but different parity, e.g. $E 1+M 3$ and $M 1+E 3$. Between these two pairs one expects an interference effect which can alter the angular distribution significantly. Such an effect was observed for the $0_{2}^{+} \rightarrow 0_{1}^{+}$-transition in ${ }^{90} \mathrm{Zr}$ (see Fig. 8 of Ref. [Kra87] and Ref. [Sch84]). The interference term is expected to have a $\cos (\theta)$ dependence [Kra87]. The angular distribution is fitted with the function $a+b \cdot \cos (\theta)$ to illustrate that this effect is in principle able to describe the data (dashed black line in Fig. 4.7).

In order to understand the angular distribution a theoretical analysis in the framework of the Quasi-Particle Phonon model is mandatory, which takes into account the contribution of giant resonances and the interference of different multipole pairs.

\subsection{Evidences for the two-photon decay}

A clearly visible peak was observed in the sum energy spectra of $72^{\circ}$ and $144^{\circ}$ at the expected energy of $662 \mathrm{keV}$. Although it was assumed in the discussion of the angular distribution, an experimental proof that this peak is indeed due to the two-photon decay and not due to 'direct' Compton scattering (see Sect. 3.1), is still missing.

The first argument against 'direct' Compton scattering as origin of the observed peak is the experimental setup with $8 \mathrm{~cm}$ to $12.5 \mathrm{~cm}$ of lead between each detector pair. The amount of lead is sufficient - according to a GEANT4 [Ago03] simulation of the experimental setup - to suppress Compton scattering reliably. This was discussed in detail in Sect. 3.1. Although this is a strong argument a more direct proof is desirable.

The five $\mathrm{LaBr}_{3}$-detectors have an excellent time resolution of $\sim 1.4 \mathrm{~ns}$ in the energy range of interest. On the one hand the time resolution is not good enough to separate 'direct' Compton scattered events from two-photon events. On the other hand it is good enough to obtain information from the shape of time spectrum whether the peak seen at $662 \mathrm{keV}$ is due to Compton scattering or due to the two-photon decay.

A sharp energy condition was imposed on the sum energy spectrum of the $72^{\circ}$-data requiring $655 \mathrm{keV}<E_{1}+E_{2}<669 \mathrm{keV}$. The corresponding time spectrum of the events fulfilling this condition are shown on the left-hand side of Fig. 4.8 (red circles), after subtraction of the random coincidences. The spectrum exhibits a time peak centered around $\Delta t=0$. In order 

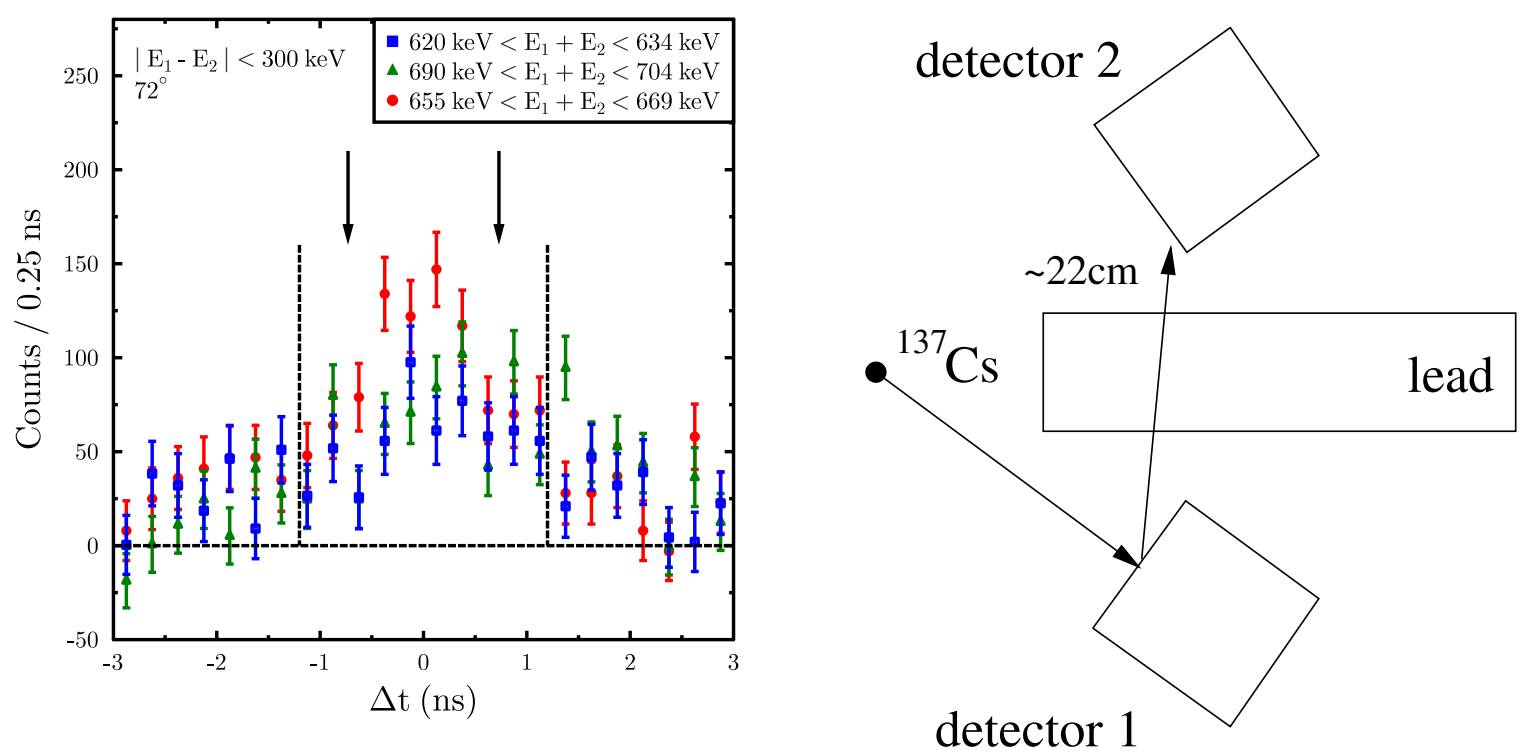

Figure 4.8: Proof for the two-photon decay using the structure of the time spectrum (left-hand side). The figure displays two time spectra with energy gates on the left-hand side (blue squares) and on the right-hand side (green triangles) of $662 \mathrm{keV}$ to obtain the time spectrum of the background. Both time spectra have a similar shape. The time spectrum with a sharp gate on the two-photon peak (red circles) exhibits a clear peak centered around $\Delta t=0$. In case of 'direct' Compton scattering one would expect two peaks at the positions marked with black arrows. On the right-hand side the average path of a 'direct' Compton scattered photon is presented (black arrows)

to rule out the possibility that this peak is due to contributions from the background that is present without a radioactive source, two energy gates were set on the left-hand and righthand side of the $662 \mathrm{keV}$ peak. They require $620 \mathrm{keV}<E_{1}+E_{2}<634 \mathrm{keV}$ (blue squares) and $690 \mathrm{keV}<E_{1}+E_{2}<704 \mathrm{keV}$ (green triangle). Since the background is not constant in this energy region - the two time spectra belonging to the background gates - were scaled with an appropriate factor determined from the exponential function describing the background in Fig. 4.1. Both background time spectra match within the uncertainties. Clearly, the time peak seen in the spectrum belonging to the energy gate on the peak at $662 \mathrm{keV}$ is not caused by the background.

The average distance which has to be traveled by a Compton scattered photon to reach a second detector is $\sim 22 \mathrm{~cm}$ (solid black arrows on the right-hand side of Fig. 4.8). Hence one expects for 'direct' Compton scattering a time spectrum with a double peak structure with centroids at \pm 0.73 ns. The centroid positions are marked in Fig 4.8 (left-hand side) with black arrows. Clearly, the measured time spectrum does not exhibit this shape. One observes a time peak with a centroid at $\Delta t=0$. This rules out 'direct' Compton scattering as (main) origin of the $662 \mathrm{keV}$ peak.

The $11 / 2^{-}$-state at $662 \mathrm{keV}$ is the second excited state in ${ }^{137} \mathrm{Ba}$. The first excited state is a $1 / 2^{+}$-state at $284 \mathrm{keV}$. It is possible that the $662 \mathrm{keV}$ peak observed in the sum energy spectrum is due to the cascade $11 / 2^{-} \rightarrow 1 / 2^{+} \rightarrow 3 / 2^{+}$with two emitted photons with energies of $378 \mathrm{keV}$ and $284 \mathrm{keV}$. This can be easily tested with energy gates around both energies. Events with one energy within these energy gates are not considered. The resulting sum energy spectrum (not 
shown here) is similar to the one obtained without these energy gates. Within the uncertainties the $662 \mathrm{keV}$ peak areas agree with each other. This suggests that the $11 / 2^{-} \rightarrow 1 / 2$-transition which corresponds to a E5-transition - is significantly weaker than the two-photon decay.

Therefore the only remaining possibility for the peak seen at $662 \mathrm{keV}$ is indeed the two-photon decay.

\subsection{The energy sharing distribution}

The energy sharing distribution $d \Gamma_{\gamma \gamma} / d \omega$ is an other important quantity to obtain deeper insights in the properties of the two-photon decay. This distribution was discussed in Sect. 2.2 and has a $d \Gamma_{\gamma \gamma} / d \omega \sim \omega^{5}(662-\omega)^{5}$ dependence in case of a pure quadrupole-quadrupole transition and $d \Gamma_{\gamma \gamma} / d \omega \sim \omega^{3}(662-\omega)^{7}+\omega^{7}(662-\omega)^{3}$ dependence for pure dipole-octupole. The $72^{\circ}$-data was divided in five energy bins with a width of $50 \mathrm{keV}$ each. This was accomplished by imposing the following energy conditions $\left|E_{1}-E_{2}\right|<50 \mathrm{keV}, 50 \mathrm{keV}<\left|E_{1}-E_{2}\right|<100 \mathrm{keV}$, $100 \mathrm{keV}<\left|E_{1}-E_{2}\right|<150 \mathrm{keV}, 150 \mathrm{keV}<\left|E_{1}-E_{2}\right|<200 \mathrm{keV}$ and $200 \mathrm{keV}<\left|E_{1}-E_{2}\right|<250 \mathrm{keV}$. These correspond to the following energy regions $306-356 \mathrm{keV}, 281-306 \mathrm{keV}$ and 356-381 keV, 256-306 keV and 381-406 keV, 256-231 keV and 406-431 keV, 231-206 keV and 431-456 keV, respectively. The regions and the theoretical distributions are shown on the left-hand side of Fig. 4.9 (note that the energy sharing distributions is symmetrical around $331 \mathrm{keV}$ ).
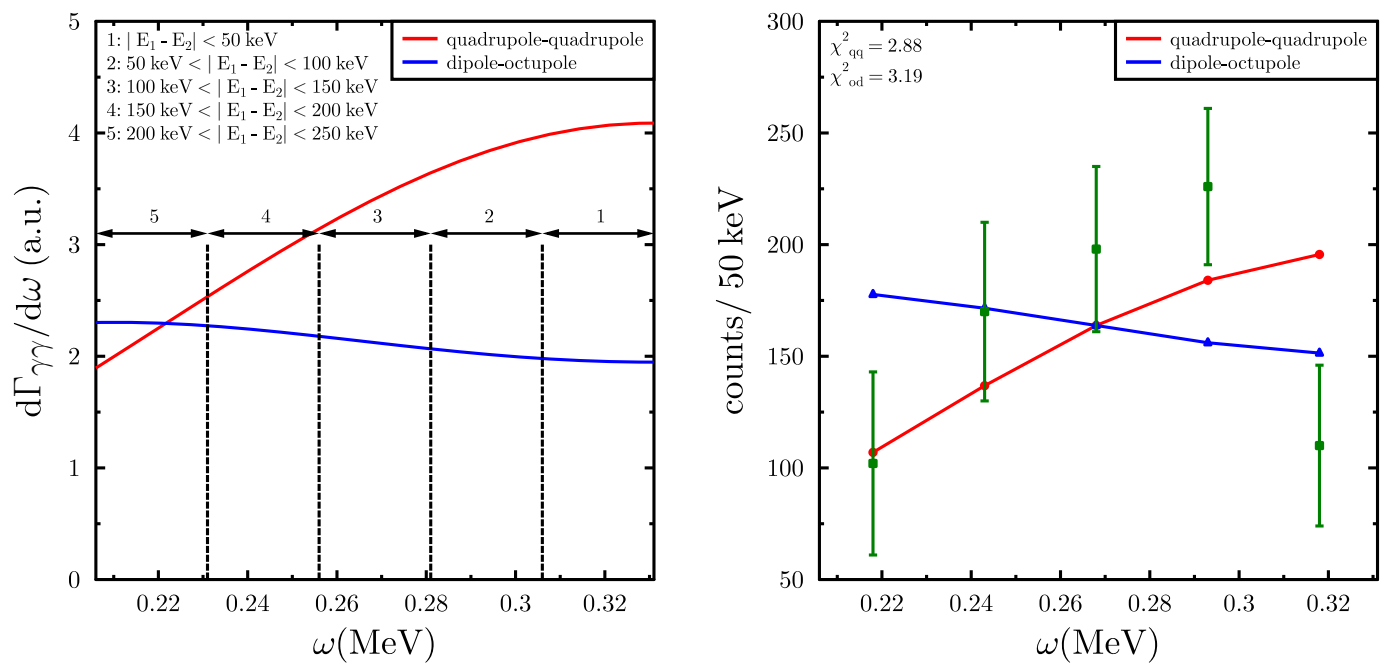

Figure 4.9: The two-photon decay probability in dependence of the energy of the photon with the lower energy. On the left-hand side the measured energy range is displayed which is divided in five regions corresponding to the regions chosen in Fig. 4.10. A comparison between experiment and theory is shown on the right-hand side.

In order to measure the energy sharing distribution the sum energy spectra were created imposing the five conditions (see Fig. 4.10). The background was fitted individually for each spectrum with the same exponential function than the full $72^{\circ}$-data in Sect. 4.4. The two-photon peak was fitted with a Gaussian function with a fixed width and fixed centroid position. The $\chi_{\text {red }}^{2}$-value is for all five spectra reasonable.

The resulting energy sharing distribution is displayed on the right-hand side of Fig. 4.9 as a function of the energy $\omega$ of the photon with the lower energy. It increases monotonically for the 
first four energies bins and drops by more than a factor two from the $50 \mathrm{keV}<\left|E_{1}-E_{2}\right|<100 \mathrm{keV}$ bin to the $\left|E_{1}-E_{2}\right|<50 \mathrm{keV}$-bin. At first glance this drop seems to be surprising. Indeed some irregularity can be found in the corresponding energy spectrum. At energies of $\sim 620 \mathrm{keV}$ and $\sim 690 \mathrm{keV}$ is an accumulation of counts which is not understood. Nevertheless these structures are separated in energy from the $662 \mathrm{keV}$ peak and there is no reason to assume that the data point of the $\left|E_{1}-E_{2}\right|<50 \mathrm{keV}$-bin is incorrect. Care was taken that the energy bins at $\sim 620 \mathrm{keV}$ and $\sim 690 \mathrm{keV}$ do not 'pull up' the background.
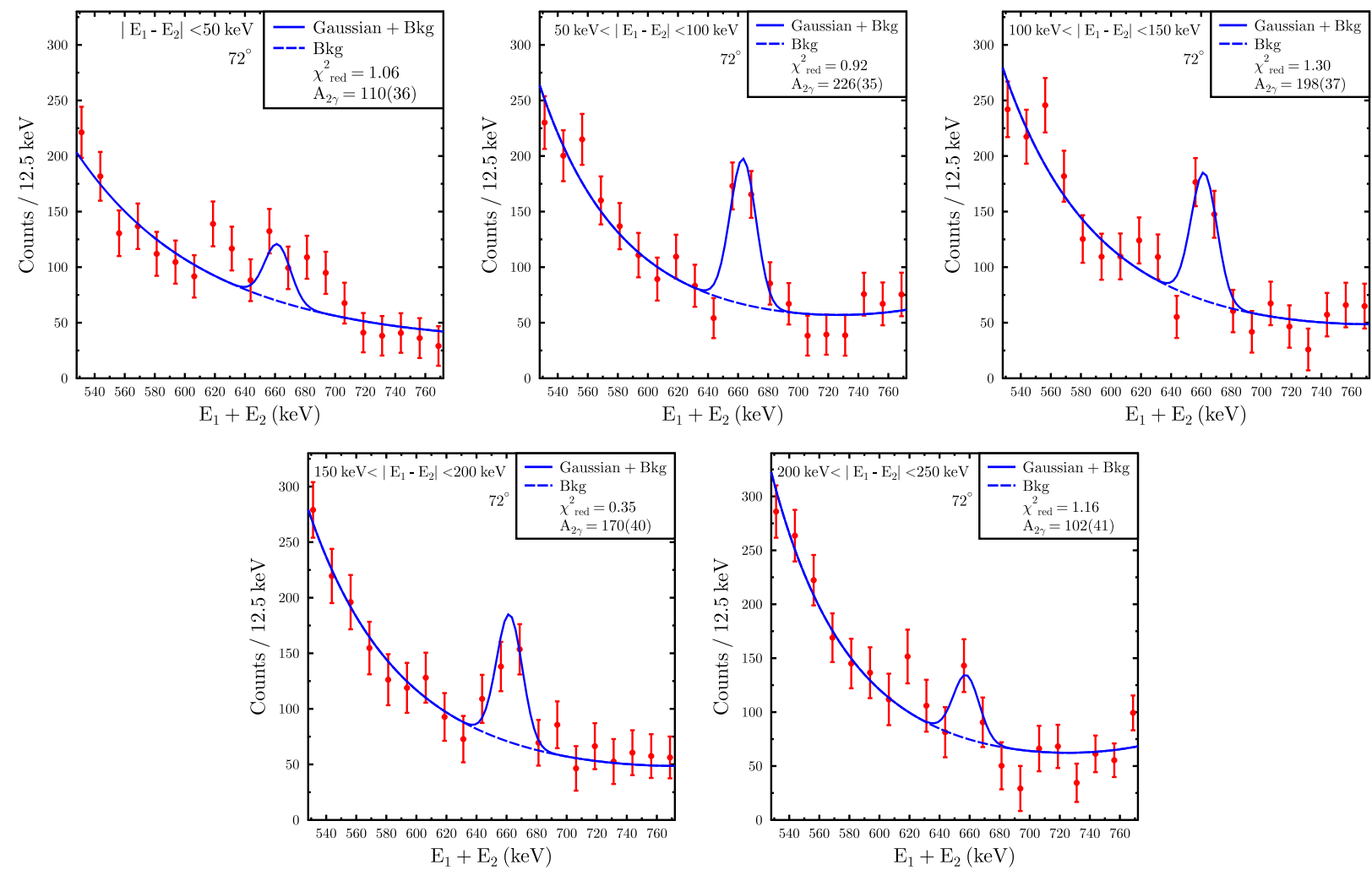

Figure 4.10: Same as the figure on the right-hand side of Fig. 4.5 but with different energy conditions.

The measured energy distribution is compared to the theoretical predictions for pure multipole transitions. The quadrupole-quadrupole energy distribution as well as the dipole-octupole distribution are fitted to the measured distribution using on overall scaling factor as the only free parameter. The $\chi_{\text {red }}^{2}$-values are unsatisfactory for both cases with $\chi_{\text {red }}^{2}=2.88$ for quadrupolequadrupole and $\chi_{\text {red }}^{2}=3.19$ for dipole-octupole. This is in agreement with the angular distribution that does not point to a pure multipole transition. Furthermore, a superposition of both multipole transitions does not improve the $\chi_{\text {red }}^{2}$-value. One should note that the discrepancies between the quadrupole-quadrupole distribution and the experimental distribution is solely due to the data point for the $\left|E_{1}-E_{2}\right|<50 \mathrm{keV}$-bin. Due to the lower statistics it was not possible the perform the same analysis for the $144^{\circ}$-data.

Clearly, in order to understand the energy sharing and the angular distributions, an analysis in a sophisticated nuclear structure model is necessary, as for example the QPM. 


\section{Theoretical background of the proton scattering experiments}

This chapter outlines the relevant theoretical background needed to understand and interpret the measured proton scattering data. This includes a discussion of the proton scattering formalism and the effective interaction utilized to account for the interaction between projectile and target in Sect. 5.1. The basics of the Interacting Boson Model 2 (IBM-2) are discussed in Sect. 5.2 with special emphasis on mixed-symmetry states. The properties of the nuclei of interest were calculated in the framework of the QPM which is covered in Sect. 5.3.

\subsection{Proton scattering}

One of the main advantages of experiments with electromagnetic probes like electron scattering or gamma spectroscopy is that our good understanding of the electromagnetic interaction which allows to give a detailed description of the reaction mechanism. Therefore observables like transition strengths and electron scattering form factors can be calculated reliably and a comparison to experimental values provide a stringent test for nuclear structure models.

For hadronic probes the situation is entirely different since the not completely understood nuclear force contributes to the excitation mechanism. One can not be sure if a possible discrepancy between the predictions of a nuclear structure model and the experimental data is due to a incorrect description of the reaction mechanism or because of shortcomings of the nuclear structure model. The situation gets even more complicated when the assumed interaction between projectile and target nucleus has several free parameters which have to be fixed phenomenologically. This gives a certain 'freedom' in the choice of these parameters thereby loosing the ability to ascribe if a certain effect is due to properties of the projectile-target interaction or a nuclear structure effect. This is in particular the case for the widely used phenomenological optical potentials.

Ideally one would like to use a framework which unifies the description of well-bound states of the target nucleus and scattering and reaction theory starting from one realistic hamiltonian based on first principles. Recent advances in $a b$ initio nuclear structure theory has made this possible for light nuclei [Hup13] and even ${ }^{40} \mathrm{Ca}$ [Hag12]. For heavy nuclei, however, these approaches are not yet feasible. Therefore in the present work the microscopic effective nucleonnucleus interaction of Refs. [Lov81, Lov85] were used while the nuclear structure input is provided by the QPM. The interaction had success in describing the proton scattering cross sections of low-lying states in different mass and energy regions. It is phenomenological and makes several approximations which are further discussed in Sect. 5.1.2. Furthermore the optical potential (see Sect. 5.1.3) is generated from this interaction, i.e. no free parameter are introduced to bridge the gap between nuclear wave functions and the theoretical proton scattering cross section.

This raises the question on how dependent the calculated cross sections are on the choice of the effective projectile-target interaction. The author of Ref. [Hof03] made a systematic 
comparison of three common interactions - including the one used in this thesis - for low-lying natural parity states in the nucleus ${ }^{58} \mathrm{Ni}$. The calculated cross section shapes were similar and the magnitudes differed by at most $\sim 30 \%$ in the region of the first maximum. This result encourages to consider proton scattering as a sensitive tool to test nuclear wave functions (in these limits) even if the $a b$ initio framework is not available yet. Especially the conclusions drawn in Sect. 8.3 are mostly independent of the interaction since they rely on the shapes of the cross sections.

Details on the calculation of the proton scattering cross sections and the made approximations are given in the next sections.

\subsubsection{Distorted wave Born approximation}

When a proton with an energy of several $\mathrm{MeV}$ interacts with the target nucleus a large number of nuclear reactions are energetically allowed including direct and compound nucleus reactions. Due to the large dimensionality of this many-body problem it is not possible to take all open reaction channels into account. One therefore has to choose a subset of the full Hilbert space which includes the relevant channels important for the description of the problem of interest. The effective hamiltonian in this model space has to account for the contributions from all channels not considered explicitly. In particular it has to have imaginary parts to model how flux is lost from the channels of the model space to other channels. This was formalized by Feshbach in Ref. [Fes58].

The effective non-relativistic hamiltonian which describes the eigenvalue problem in the model space important for inelastic proton scattering can be written as

$$
\mathbf{H}=\mathbf{H}_{\mathrm{N}}+\mathbf{T}+\mathrm{V}
$$

$\mathbf{H}_{\mathrm{N}}$ is the nuclear hamiltonian accounting for the internal structure of the nucleus, $\mathbf{T}$ is the kinetic energy operator of the relative motion and $\mathbf{V}$ stands for the effective interaction between target nucleus and projectile. The effective potential $\mathbf{V}$ can be separated into two terms $\mathbf{V}=\mathbf{U}+\mathbf{W}$ such that $\mathbf{U}$ acts only on the coordinates of the incident particle.

If one takes into account the proper antisymmetrization between the projectile and each and every target nucleon of the same type the general transition matrix is given by [Lev63, Amo67]

$$
T_{\mathrm{fi}}=f-N \cdot g,
$$

with

$$
\begin{aligned}
& f=\left\langle\chi_{\mathrm{f}}^{(-)}(0) \psi_{f}\left(\xi_{0}\right)\left|\mathbf{V}_{0}-\mathbf{U}_{0}\right| \Psi_{\mathrm{i}}^{(+)}\left(0, \xi_{0}\right)\right\rangle, \\
& g=\left\langle\chi_{\mathrm{f}}^{(-)}(1) \psi_{f}\left(\xi_{1}\right)\left|\mathbf{V}_{1}-\mathbf{U}_{1}\right| \Psi_{\mathrm{i}}^{(+)}\left(0, \xi_{0}\right)\right\rangle .
\end{aligned}
$$

$N$ is the number of particles which are identical with the projectile. The terms $f$ and $g$ are direct and exchange transition matrix elements. The exchange term is known to be important for the correct description of $T_{\mathrm{fi}}$ and therefore included in all calculations in this work. The incoming/outgoing $( \pm)$ 'distorted waves' $\chi^{( \pm)}(k)$ of particle $k$ are solutions of the Schroedinger equation with the single particle potential $\mathbf{U}_{\mathrm{k}}$. $\xi_{0}$ is a set of nuclear quantum numbers $1,2, \ldots, A$ 
and $\xi_{1}$ is the set $0,2, \ldots, A . \mathrm{V}_{\mathrm{k}}$ describes the interaction between nucleon $k$ and all other nucleons and is assumed to have a two-body character i.e.

$$
\mathbf{V}_{\mathrm{k}}=\sum_{\mathrm{j}} \mathbf{V}(k, j)
$$

The wave function $\Psi_{0}^{(+)}\left(j, \xi_{0}\right)$ in Eq. 5.3 is the unknown exact solution of the full Hamiltonian of Eq.5.1 which is - in the relative coordinates of projectile and target nucleus - asymptotically a plane wave plus scattered spherical waves in all energetically allowed reaction channels.

In order to calculate the transition matrix element one has to find a suitable approximation for this wave function. First of all one can restrict $\Psi_{0}^{(+)}\left(j, \xi_{0}\right)$ to the channel of interest thereby neglecting the coupling to other channels and possible multi-step processes. Secondly one can expand the wave function of the relative motion in a Born series [Jac70] and consider only the first term $\chi^{+}(k)$. This scheme is called distorted wave Born approximation (DWBA) and $\Psi_{\mathrm{i}}^{(+)}\left(j, \xi_{j}\right)$ in Eq. 5.3 is replaced through $\left|\Psi_{\mathrm{i}}^{(+)}\left(0, \xi_{0}\right)\right\rangle \approx\left|\psi_{i} \chi_{\mathrm{i}}^{+}(0)\right\rangle$. By a co-factor expansion of the nucleus wave functions $\psi_{i(f)}$ the DWBA transition matrix element $T_{\mathrm{fi}}$ can be converted to [Ger71]

$$
\begin{aligned}
& T_{\mathrm{fi}}=\sum_{j_{1} j_{2} m_{1} m_{2}}\left\langle\psi_{f}\left|\mathbf{a}_{j_{2} m_{2}}^{\dagger} \mathbf{a}_{j_{1} m_{1}}\right| \psi_{i}\right\rangle \\
& \cdot\left\langle\chi_{\mathrm{f}}^{(-)}(0) \phi_{j_{2} m_{2}}(1)|\mathbf{V}(0,1)| \chi_{\mathrm{i}}^{(+)}(0) \phi_{j_{1} m_{1}}(1)-\chi_{\mathrm{i}}^{(+)}(1) \phi_{j_{1} m_{1}}(0)\right\rangle .
\end{aligned}
$$

The transition matrix element $T_{\mathrm{fi}}$ is a weighted sum of all inelastic scattering amplitudes where a single nucleon in the bound state $\phi_{j_{1} m_{1}}$ with angular moment $j_{1}$ and projection $m_{1}$ is excited to a state with quantum number $j_{2}$ and $m_{2}$. The weighting factors $\left\langle\psi_{f}\left|\mathbf{a}_{j_{2} m_{2}}^{\dagger} \mathbf{a}_{j_{1} m_{1}}\right| \psi_{i}\right\rangle$ are determined by the structure of the nuclear wave function in the initial and final state (they are closely related the summands in Eq. 5.36). Equation 5.5 considers only excitation mechanisms which promote a single nucleon. This is usually justified since the excitation probability of two or more nucleons is much smaller.

The theoretical cross section - which is then compared to the experimental one - can be calculated from the square of $T_{\mathrm{fi}}$ and proper normalization factors. A detailed expression is given in Ref. [Amo67]. In the present work the transition matrix elements $T_{\mathrm{fi}}$ and the corresponding cross sections were calculated with the computer code DWBA07 [Ray07a] developed by J. Raynal. Details on the calculation procedure are outlined in Ref. [Ray07b]. For calculating the total transition matrix $T_{\mathrm{fi}}$ three basic ingredients have to be specified

- the nuclear wave functions and the properties of the single nucleon states have to be known,

- the optical potential $\mathbf{U}$ has to specified from which the distorted waves are generated,

- and one has to choose an effective interaction $\mathrm{V}(0,1)$.

The nuclear structure input were calculated in the framework of the QPM which is outlined in Sect. 5.3. The effective interaction and the optical potential are discussed in the next two sections. 


\subsubsection{The effective interaction}

The effective interaction [Lov81, Lov85] used in this work to calculate proton scattering cross sections is based upon the free nucleon-nucleon t matrix. It is very intuitive to use the free nucleon-nucleon t matrix - which is closely related to the nucleon-nucleon scattering amplitudes [Jac70] - as an effective interaction between the incident nucleon and the target nucleus. In this approximation - frequently called impulse approximation - the scattering process is assumed to be a sum of single scatterings involving one target nucleon at a time with an mutual interaction which is determined from the free scattering, i.e. it is in principle known from nucleon-nucleon scattering experiments. However this approximation neglects that both nucleons scatter in the medium.

For computational feasibility the authors of Refs. [Lov81, Lov85] represent the free $t$ matrix by a local, energy-independent coordinate space interaction of the following form

$$
\mathbf{V}(0,1)=V^{C}(r)+V^{L S}(r) \overrightarrow{\mathbf{L}} \cdot \overrightarrow{\mathbf{S}}+V^{T}(r) \mathbf{S}_{12}
$$

$\overrightarrow{\mathbf{L}}$ is the angular momentum of the relative motion, $\overrightarrow{\mathbf{S}}=\frac{1}{2}\left(\vec{\sigma}_{1}+\vec{\sigma}_{2}\right)$ is the spin operator and $\vec{\sigma}_{i}$ are the Pauli matrices. The tensor operator is given through $\mathbf{S}_{12}=3\left(\vec{\sigma}_{1} \cdot \vec{r} / r\right)\left(\vec{\sigma}_{2} \cdot \vec{r} / r\right)-\vec{\sigma}_{1} \cdot \vec{\sigma}_{2}$. The radial dependence of $V^{C}(r)$ and $V^{L S}(r)$ are approximated by Yukawa forms $Y(x)=e^{-x / x}$ reflecting the exchange of mesons

$$
V^{\alpha}(r)=\sum_{i=1}^{N_{\alpha}} V_{i}^{\alpha} \cdot Y\left(r / R_{i}\right),
$$

and the radial shape of the tensor part is assumed to be

$$
V^{T}(r)=\sum_{i=1}^{N_{T}} V_{i}^{T} \cdot r^{2} \cdot Y\left(r / R_{i}\right) .
$$

The $R_{i}$ are the range parameters of the interaction. The complex $V$ coefficients are adjusted until the free nucleon-nucleon $t$ matrix calculated with the 'pseudo' potential of Eq. 5.6 give a reasonable description to the experimental one obtained from phase-shift analysis. Several other constraints were applied to the interaction which are motivated by the one-pion-exchange potential [Lov81].

The chosen method has several shortcomings. It neglects in-medium effects like Fermi averaging or Pauli blocking and no constraints were applied to the off-energy-shell properties of the free $t$ matrix [Els89]. Today more advanced effective interactions are available which account for the mentioned effects e.g. the one of Ref. [Kar95] which is based on the nucleon-nucleon $g$ matrix. Nevertheless the interaction used in the present work allows a reasonable description of proton scattering data at energies larger than $\sim 150 \mathrm{MeV}$ where the impulse approximation is valid [Lov81].

\subsubsection{Microscopic optical model}

The optical potential $\mathbf{U}_{\mathrm{i}}$ in Eq. 5.3 accounts for the interaction between the projectile and the target in the entrance and exit channels. Often its parameters are fixed phenomenologically to 
give a good description for the elastic scattering cross section. With such an optical potential a 'semi-microscopic' calculation is then performed for inelastic scattering [Gaz83] where the optical potential is without any microscopic foundation while a microscopic transition operator - like the one discussed in Sect. 5.1.2 - is used to excite the nucleus. In this thesis it was tried to stay as microscopic as possible. The effective interaction of Sect. 5.1.2 was used as an transition operator as well as to generate the optical potential. This is done by folding the ground state density - calculated in the framework of the QPM - with the effective interaction [Rik84]. The resulting real and imaginary parts of the central and spin-orbit potentials are shown in Fig. 5.1.

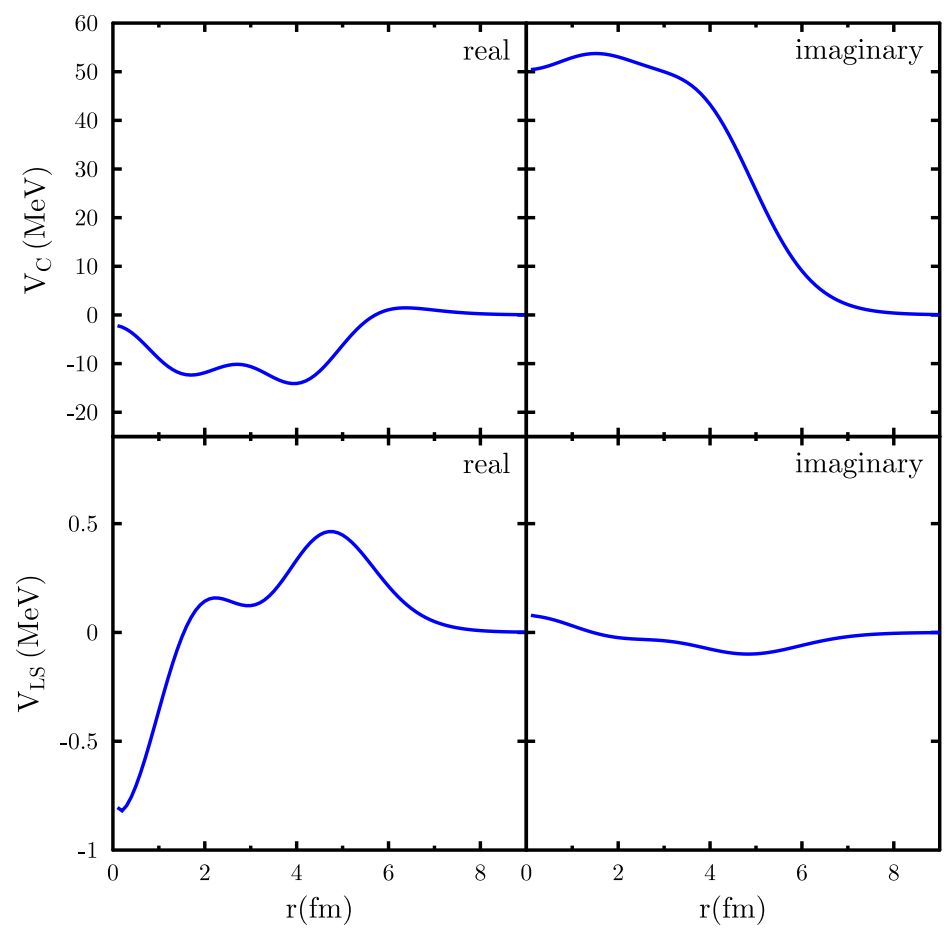

Figure 5.1: The calculated microscopic optical potentials found by folding the ground state density of ${ }^{92} \mathrm{Zr}$ with the effective interaction.

\subsection{Mixed-symmetry states in the Interacting Boson Model 2}

Originally so called mixed-symmetry states were defined in the framework of the IBM-2. It is the purpose of this section to give a brief overview of the IBM-2 and to present the definition of a mixed-symmetry state. Also discussed are its main experimental signatures. This section follows Refs. [Pie08, Wal10].

The IBM-2 is a phenomenological approach to collective low-lying excitations in the valence shell. Its effective degrees of freedoms are a fixed number of bosons which can be considered as correlated nucleon pairs of the same type. Usually the model space in even-even nuclei is restricted to $s$ - and $d$-bosons only - that are bosons with angular moment $J=0^{+}$and $J=2^{+}$ respectively. This is motivated by the fact that the pairing force is strongest in the $J=0$ and $J=2$ channels. In contrast to the IBM- 1 the IBM-2 distinguishes between proton and neutron bosons. The model space of the nuclear shell model increases drastically when going from magic 
nuclei to open-shell systems leading to huge matrices with dimensions of the order of $\sim 10^{20}$ where diagonalization is not feasible. The IBM-2 can be seen as a drastic and rough truncation of this model space - neglecting fermionic degrees of freedom - hoping to pick the configurations which are important for the description of low-lying collective states. The good description of experimental data justifies this treatment of the nuclear many-body problem to a certain degree.

The general IBM-2 Hamiltonian - considering only one-body and two-body terms - can be written as [Pie08]

$$
H=E_{0}+\sum_{\alpha} \epsilon_{\alpha} \mathbf{b}_{\alpha}^{+} \tilde{\mathbf{b}}_{\alpha}+\sum_{\alpha \beta \gamma \delta} c_{\alpha \beta \gamma \delta} \mathbf{b}_{\alpha}^{+} \mathbf{b}_{\beta}^{+} \tilde{\mathbf{b}}_{\gamma} \tilde{\mathbf{b}}_{\delta}
$$

$E_{0}, \epsilon_{\alpha}, c_{\alpha \beta \gamma \delta}$ are constants, $\mathbf{b}_{\alpha}^{+}$and $\tilde{\mathbf{b}}_{\alpha}$ are boson creation and annihilation operators and $\alpha, \beta, \gamma, \delta$ codify additional quantum numbers. The Hamiltonian has to fulfill several conditions e.g. rotational symmetry and hermiticity which limits the number of terms in Eq. 5.9. Nevertheless the hamiltonian has still a large number of parameters $\sim 20$ making it difficult to perform a meaningful calculation by fixing them with a comparison to data. The key idea to reduce their number is to orientate at microscopic shell-model Hamiltonians to deduce which terms are most important. This procedure is not unique leading to several IBM-2 Hamiltonians on the market. A very common shell-model hamiltonian is the 'pairing-quadrupole hamiltonian'Its counterpart in the IBM-2 is given by [Ots85]

$$
\mathbf{H}=\epsilon_{\mathrm{p}} \mathbf{d}_{\mathrm{p}}^{\dagger} \tilde{\mathbf{d}}_{\mathrm{p}}+\epsilon_{\mathrm{n}} \mathbf{d}_{\mathrm{n}}^{\dagger} \tilde{\mathbf{d}}_{\mathrm{n}}-\kappa \mathbf{Q}_{\mathrm{p}} \mathbf{Q}_{\mathrm{n}}+\mathbf{M}_{\mathrm{pn}}
$$

where

$$
\begin{aligned}
\mathbf{Q}_{\rho} & =\mathbf{s}_{\rho}^{\dagger} \tilde{\mathbf{d}}_{\rho}+\mathbf{d}_{\rho}^{\dagger} \mathbf{s}_{\rho}+\chi_{\rho}\left[\mathbf{d}_{\rho}^{\dagger} \tilde{\mathbf{d}}_{\rho}\right]^{(2)} \\
\mathbf{M}_{\mathrm{pn}} & =\sum_{L=1}^{3} \lambda_{L}\left[\mathbf{d}_{\mathrm{n}}^{\dagger} \mathbf{d}_{\mathrm{p}}^{\dagger}\right]^{(L)}\left[\tilde{\mathbf{d}}_{\mathrm{n}} \tilde{\mathbf{d}}_{\mathrm{p}}\right]^{(L)} .
\end{aligned}
$$

The $\kappa \mathbf{Q}_{\mathrm{p}} \mathbf{Q}_{\mathrm{n}}$ term mimics the quadrupole proton-neutron interaction in the shell-model, while $\epsilon_{\rho} \mathbf{d}_{\rho}^{\dagger} \tilde{\mathbf{d}}_{\rho}$ can be seen as the IBM-2 analogon of the monopole pairing part. The constants $\epsilon_{\mathrm{p}}$ and $\epsilon_{\mathrm{n}}$ are the d-boson energies. The last part $\mathbf{M}_{\mathrm{pn}}$ is the Majorana operator which has no microscopic counterpart. The hamiltonian of Eq. 5.11 has proven to account successfully for the properties of a large number of nuclei.

One of the outstanding features of the IBM is the application of group theoretical methods to nuclear structure physics. This allows in many cases an intuitive interpretation of nuclear structure phenomena. Well known are the dynamical symmetries [Iac87] contained in the IBM-1 $U(5), S U(3)$ and $O(6)$ - where the eigenvalues of the corresponding Hamiltonian can be obtained analytically. Nuclear structure observables can be compared to these 'limiting cases' and one can draw conclusions if the nucleus of interest behaves like e.g. a $U(5)$-nucleus or a $O(6)$ nucleus. This helps significantly to understand nuclear structure and brings somehow order in the complicated nuclear landscape. The $U_{\mathrm{p}}(6) \times U_{\mathrm{n}}(6)$ group structure of the IBM- 2 - still containing $U(5), S U(3)$ and $O(6)$ of the IBM-1 - is richer in terms of dynamical symmetries [Iac87] but is not further discussed here. 
The focus of this section is on the F-Spin symmetry [Isa86] which allows to define the term 'mixed-symmetry state'In analogy to the isospin concept of nuclear physics proton and neutron bosons are considered as different states of one particle with the following quantum numbers [Pie08]

$$
\begin{aligned}
& \mathbf{b}_{\mathrm{p}}^{\dagger}|0\rangle=\left\{\begin{array}{c}
F=1 / 2 \\
F_{z}=+1 / 2
\end{array}\right. \\
& \mathbf{b}_{\mathrm{n}}^{\dagger}|0\rangle=\left\{\begin{array}{c}
F=1 / 2 \\
F_{z}=-1 / 2 .
\end{array}\right.
\end{aligned}
$$

$F_{z}$ is the projection of $F$ on the quantization axis. Mathematically $F$-Spin is identical to isospin imposing also a $S U(2)$ group structure. The generators of the $S U(2)$ group are [Pie08]

$$
\begin{aligned}
& \mathbf{F}_{+}=\mathbf{d}_{\mathrm{p}}^{+} \tilde{\mathbf{d}}_{\mathrm{n}}+\mathbf{s}_{\mathrm{p}}^{+} \mathbf{s}_{\mathrm{n}} \\
& \mathbf{F}_{-}=\mathrm{d}_{\mathrm{n}}^{+} \tilde{\mathbf{d}}_{\mathrm{p}}+\mathbf{s}_{\mathrm{n}}^{+} \mathbf{s}_{\mathrm{p}} \\
& \mathbf{F}_{z}=\frac{1}{2}\left[\mathbf{d}_{\mathrm{p}}^{+} \tilde{\mathbf{d}}_{\mathrm{p}}+\mathbf{s}_{\mathrm{p}}^{+} \mathbf{s}_{\mathrm{p}}-\mathrm{d}_{\mathrm{n}}^{+} \tilde{\mathbf{d}}_{\mathrm{n}}-\mathbf{s}_{\mathrm{n}}^{+} \mathbf{s}_{\mathrm{n}}\right]
\end{aligned}
$$

The eigenvalue of $\mathbf{F}_{z}$ is $\frac{1}{2}\left(N_{\mathrm{p}}-N_{\mathrm{n}}\right) . N_{\mathrm{p}}$ and $N_{\mathrm{n}}$ are the proton and neutron boson numbers. The effect of $\mathbf{F}_{+}$and $\mathbf{F}_{-}$on the wave function is apparent from their structure. They enhance or lower $F_{z}$ by one unit. The operators of Eq. 5.13 are close under commutation and form a Lie-Algebra. It is possible to define a Cassimir-Operator commuting with each generator:

$$
\mathbf{F}^{2}=\mathbf{F}_{-} \mathbf{F}_{+}+\mathbf{F}_{z}\left(\mathbf{F}_{z}+1\right)
$$

States with good $F$-Spin - the eigenvalue of $\mathbf{F}^{2}: F(F+1)$ - can be formed and $F_{\mathrm{z}}$ is always a good quantum number independently of the IBM-2 Hamiltonian. Consequently $F_{\mathrm{z}}$ and $F$ can be used to label the basis states. The $F$-Spin quantum number can take values between $F_{\min }=$ $\left|N_{\mathrm{p}}-N_{\mathrm{n}}\right| / 2$ and $F_{\max }=\left(N_{\mathrm{p}}+N_{\mathrm{p}}\right) / 2$. It classifies the symmetry of a wave function with respect to pairwise exchange of proton and neutron labels. In Ref. [Pie08] it is shown that states with maximum $F$-Spin $F=F_{\max }$ are symmetric under pairwise exchange of proton and neutron bosons, while states with $F<F_{\max }$ have at least one pair which is anti-symmetric. Therefore the former class of states are called fully symmetric states (FFSs) while the latter are referred to as mixed symmetry states (MSSs).

The necessary requirement for $F$-Spin being an exact symmetry is that the generators of Eq. 5.13 commute with the hamiltonian of Eq. 5.10. However $\left[\mathbf{F}_{ \pm}, \mathbf{H}\right]=0$ is not fulfilled. Moreover $\mathbf{F}^{2}$ does not even commute with $\mathbf{H}$, i.e. in principle one cannot expect $F$-Spin to be a good quantum number and therefore a useful concept. However the Majorana-Operator of Eq. 5.11 helps. It acts solely on states with $F<F_{\max }$ and gives them an overall shift to higher energies. This reduces the mixing between states with different $F$-Spin quantum numbers since the mixing matrix element depends on the energy splitting. For this reason in realistic IBM-2 calculations $F$-Spin is indeed approximately a good quantum number and can be used to understand and interpret nuclear spectra and observables. Moreover one finds so called $F$-Spin multiplet in many nuclear regions which clearly show that even $F$-Spin symmetry is not severely broken. 
In order to identify a mixed-symmetry state electromagnetic transition rates are useful observables. The M1-transition operator in the IBM-2 is defined as

$$
\mathbf{T}(M 1)=\sqrt{\frac{3}{4 \pi}}\left[g_{\mathrm{p}} \mathbf{L}_{\mathrm{p}}+g_{\mathrm{n}} \mathbf{L}_{\mathrm{n}}\right] \mu_{N}
$$

where $g_{\mathrm{p}}$ and $g_{\mathrm{n}}$ are the proton and neutron boson $g$-factors and

$$
\mathbf{L}_{\rho}=\sqrt{10}\left[\mathbf{d}_{\rho}^{+} \mathbf{d}_{\rho}\right]^{(1)},
$$

denotes the angular momentum operators. The $M 1$-operator connects only states which differ by one unit of $F$-Spin [Pie08]. Therefore $M 1$-transition between two fully-symmetric states or two mixed-symmetry states are exactly forbidden. Since states with $F<F_{\max }-1$ are at higher energies (and have actually never been experimentally verified) one can be sure that a large $M 1$-transition is between a MSS with $\mathrm{F}=\mathrm{F}_{\max }-1$ and a FSS giving a reliable experimental signature to identify the former class of states.

Additional valuable information can be obtained from E2-transitions. The E2-transition operator is

$$
\mathbf{T}(E 2)=e_{\mathrm{p}} \mathbf{Q}_{\mathrm{p}}^{\chi_{\mathrm{p}}}+e_{\mathrm{n}} \mathbf{Q}_{\mathrm{n}}^{\chi_{\mathrm{n}}}
$$

where $e_{\mathrm{p}}$ and $e_{\mathrm{n}}$ are boson effective charges accounting for states which are not included in the small IBM-2 model space but contribute to the transition. If $F$-Spin symmetry is valid E2- and M1-transition matrix elements can be written as [Isa86]

$$
\begin{aligned}
\langle\mathrm{FSS}\|\mathrm{T}(M 1)\| \mathrm{MSS}\rangle & \sim\left(g_{\mathrm{p}}-g_{\mathrm{n}}\right) \sqrt{N_{\mathrm{p}} N_{\mathrm{n}}} \\
\langle\mathrm{FSS}\|\mathrm{T}(E 2)\| \mathrm{FSS}\rangle & \sim e_{\mathrm{p}} N_{\mathrm{p}}+e_{\mathrm{n}} N_{\mathrm{n}} \\
\langle\mathrm{FSS}\|\mathrm{T}(E 2)\| \mathrm{MSS}\rangle & \sim\left(e_{\mathrm{p}}-e_{\mathrm{n}}\right) \sqrt{N_{\mathrm{p}} N_{\mathrm{n}}} .
\end{aligned}
$$

Since the $g$-factor are usually taken to be $g_{\mathrm{p}}=1$ and $g_{\mathrm{n}}=0$ and therefore $g_{\mathrm{p}}-g_{\mathrm{n}}=1$ the $M 1$ matrix element is of the order of $\langle\mathrm{FSS} \| \mathrm{T}(M 1)|| \mathrm{MSS}\rangle \approx 1 \mu_{\mathrm{N}}$. The E2-matrix element connecting two FSSs is collective and of the order of several W.u. because proton and neutron parts sum up coherently. This is in contrast to the $\langle\mathrm{FSS}|| \mathrm{T}(E 2)|| \mathrm{MSS}\rangle$ matrix element where the proton and neutron contributions cancel to some degree. This matrix element is expected to be of the order of $\sim 1$ W.u.

Figure 5.2 summarizes the expected level structure and decay properties of the low-lying collective states of a spherical nucleus in the framework of the IBM-2. Boson numbers of $N_{p}=$ $N_{n}=1$ and a schematic IBM-2 hamiltonian of the form $H=\epsilon\left(N_{\mathrm{p}}+N_{\mathrm{n}}\right)+\lambda M$ with $N_{\mathrm{p}}=N_{\mathrm{n}}=1$ and $M=\left(F_{\max }\left(F_{\max }+1\right)-F(F+1)\right)$ are assumed. The Majorana-Operator $M$ acts only on states with $F<F_{\max }$ and shifts them to higher energies in dependence of the parameter $\lambda$. Boson wave functions and transition strengths are also given, exhibiting the discussed properties. The symmetric two-phonon triplet with $J^{\pi}=0^{+}, 2^{+}, 4^{+}$and its mixed-symmetric counterpart $J^{\pi}=$ $1^{+}, 3^{+}$are shown. A larger number of boson would expand the allowed angular momentum values of the latter one to $J^{\pi}=0^{+}, 1^{+}, 2^{+} 3^{+}, 4^{+}$.

The $Q$-phonon scheme [Ots94, Kim96] makes an other feature of the $2_{1}^{+}$and $2_{\mathrm{ms}}^{+}$states apparent. They act as building blocs of collective nuclear structure forming the symmetric and mixed-symmetric two-phonon multiplets. This highlights the importance to study and understand the properties of the $2_{\mathrm{ms}}^{+}$state. The main focus of this work is on the mixed-symmetric one-phonon quadrupole state. The properties of $2_{\mathrm{ms}}^{1}$ state can be summarized as: 


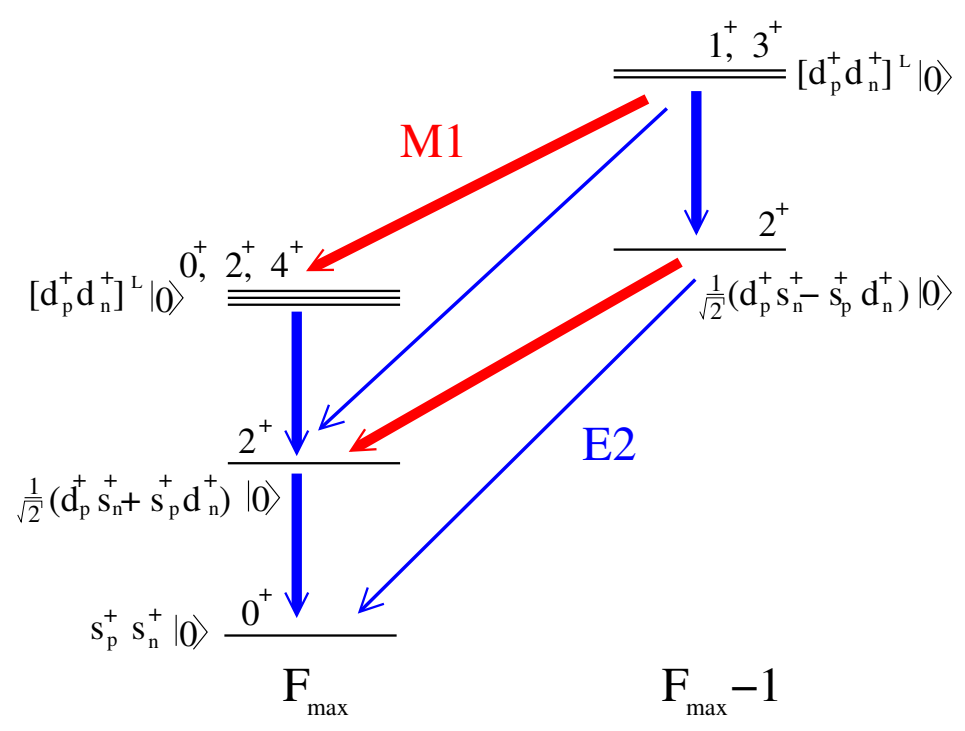

Figure 5.2: The low-lying states and their decay properties of a spherical nucleus in the framework of the IBM-2 with boson numbers $\mathrm{N}_{p}=\mathrm{N}_{n}=1$. The thickness of the arrows indicate the strength of the transition.

- a large $M 1$-transition to the $2_{1}^{+}$state of the order of $\sim 1 \mu_{N}$

- a weakly collective E2-transition with a strength of $\sim 1$ W.u. to the ground state

\subsection{The Quasiparticle Phonon Model}

\subsubsection{Outline of the model}

An introduction to the QPM - used to calculate the properties of the nuclei ${ }^{92} \mathrm{Zr},{ }^{94} \mathrm{Zr}$ and ${ }^{94} \mathrm{Mo}$ - is given in this section which follows closely the literature [Ber99, Wal10]. The QPM is a purely phenomenological but microscopic approach to nuclear structure. It tackles the nuclear many body problem by making several simplifying assumptions which allow to cover a large configuration space, i.e. no effective charges are necessary, and to include the coupling between one-, two- and three-phonon states. The QPM is able to describe the properties of low-lying states and the high energy response at the same time.

There are two 'versions' of the model on the market. The first one starts from a deformed Wood-Saxon mean field potential and is able to describe the properties of deformed nuclei [Sol92], while the second version - used in the present work - uses a spherical basis and is able to describe nuclei with a few valence nucleons. Transitional nuclei are so far not in the reach of the QPM.

The QPM approximates the nuclear many-body problem with the following Hamiltonian

$$
\mathbf{H}_{\mathrm{qpm}}=\mathbf{H}_{\mathrm{sp}}+\mathbf{H}_{\mathrm{pair}}+\mathbf{H}_{\mathrm{m}}^{\mathrm{ph}}+\mathbf{H}_{\mathrm{m}}^{\mathrm{pp}}+\mathbf{H}_{\mathrm{sm}}^{\mathrm{ph}}
$$

where 
- $\mathbf{H}_{\mathrm{sp}}$ is the one-body Hamiltonian usually taken to be a Wood-Saxon potential,

- $\mathbf{H}_{\text {pair }}$ accounts for monopole pairing,

- $\mathbf{H}_{\mathrm{m}}^{\mathrm{ph}}$ is a sum over separable multipole interactions in the particle-hole channel,

- $\mathbf{H}_{\mathrm{m}}^{\mathrm{pp}}$ is a separable multipole interaction in the particle-particle channel,

- $\mathbf{H}_{\mathrm{sm}}^{\mathrm{ph}}$ is a separable spin-multipole interaction in the particle hole channel.

The $\mathbf{H}_{\mathrm{sm}}^{\mathrm{ph}}$-part is only sensitive to states of unnatural parity which were not measured in the present experiment. In Ref. [Iud04] it was shown that the $\mathbf{H}_{\mathrm{m}}^{\mathrm{pp}}$-part has some influence on the properties of quadrupole states. However in the present QPM calculation this part is assumed to be zero which is debatable. Hence both Hamiltonian parts are not included in the further discussion. The Hamiltonian is diagonalized in a step-by-step procedure. Each of these steps is discussed in the following at the example of ${ }^{92} \mathrm{Zr}$ and is limited to the case of even-even nuclei.

It is well known that a good portion of the long-range correlations of the nuclear force can be absorbed by introducing an appropriate mean-field potential. In the QPM this mean-field is chosen to be a Wood-Saxon potential of the form [Ber99]:

$$
U^{\tau}(r)=\frac{V_{0}^{\tau}}{1+e^{\left(r-R_{0}^{\tau}\right) / a_{0}^{\tau}}}-\frac{\hbar^{2}}{\mu^{2} c^{2}} \frac{1}{r} \frac{d}{d r}\left(\frac{V_{l s}^{\tau}}{1+e^{\left(r-R_{l s}^{\tau}\right) / a_{l s}^{\tau}}} \mathbf{l} \cdot \mathbf{s}\right)+V_{c}(r) .
$$

$V_{c}(r)$ stands for the Coulomb potential, $\mu$ is the reduced mass and $\tau$ denotes whether the WoodSaxon potential is either for neutron or protons. The parameters of the potential are fitted to properties of nuclei of a given mass region with the restrictions of $R_{\mathrm{ls}}^{\tau}=R_{0}^{\tau}, a_{\mathrm{ls}}^{\tau}=a_{\mathrm{ls}}^{\tau}$ and $R_{\mathrm{C}}=R_{0}^{p}$. The QPM calculations for the nuclei ${ }^{92,94} \mathrm{Zr}$ and ${ }^{94} \mathrm{Mo}$ - which are all of the same mass region - are done with the parameters shown in Tab. 5.1, i.e. all nuclei have the same single particle spectra which is shown in Fig. 5.3. It was necessary, however, to lower the energy of the neutron $2 d_{5 / 2}$ single particle state in ${ }^{94} \mathrm{Zr}$ artificially by $200 \mathrm{keV}$ to account for the large $B(E 2)$-value of the $2_{2}^{+}$state (see Sect. 8.1.2). All other single particle states remain unchanged.

The strongest residual interaction* in nuclei is monopole pairing. The QPM assumes the pairing force $H_{\text {pair }}$ to be of zero-range and state independent [Ber99]

$$
\mathbf{H}_{\text {pair }}=-\sum_{\tau}^{n, p} G_{\tau}^{(0)} \sum_{j, j^{\prime}} \sqrt{(2 j+1)\left(2 j^{\prime}+1\right)}\left[\mathbf{a}_{j m}^{+} \mathbf{a}_{j-m}^{+}\right]_{00}\left[\mathbf{a}_{j^{\prime}-m^{\prime}}^{+} \mathbf{a}_{j^{\prime} m^{\prime}}^{+}\right]_{00}
$$

where

$$
\left[\mathbf{a}_{j}^{+} \mathbf{a}_{j^{\prime}}^{+}\right]_{\lambda \mu}=\sum_{m, m^{\prime}} C_{j m j^{\prime} m^{\prime}}^{\lambda \mu} \mathbf{a}_{j m}^{+} \mathbf{a}_{j^{\prime} m^{\prime}}^{+}
$$




\begin{tabular}{cccccc}
\hline \hline & $V_{0}(\mathrm{MeV})$ & $R_{0}(\mathrm{fm})$ & $a_{0}(\mathrm{fm})$ & $V_{l s}(\mathrm{MeV})$ & \\
\hline $\begin{array}{c}\text { neutrons } \\
\text { protons }\end{array}$ & -44.70 & 5.802 & 0.6200 & -9.231 & \\
\hline & -56.86 & 5.577 & 0.6301 & -9.609 & \\
\hline${ }^{(0)}$ & $G_{p}^{(0)}$ & $\kappa_{0}^{2^{+}}$ & $\kappa_{0}^{3^{-}}$ & $\kappa_{0}^{4^{+}}$ \\
& $(\mathrm{MeV})$ & $(\mathrm{MeV})$ & $(\mathrm{MeV})$ & $(\mathrm{MeV})$ & $(\mathrm{MeV})$ \\
\hline${ }^{92} \mathrm{Zr}$ & 0.168 & 0.194 & 0.018 & 0.017 & 0.020 \\
${ }^{94} \mathrm{Zr}$ & 0.168 & 0.194 & 0.017 & 0.017 & 0.018 \\
${ }^{\mathrm{Mo}}$ & 0.168 & 0.194 & 0.017 & 0.018 & 0.016 \\
\hline \hline
\end{tabular}

Table 5.1: Parameters of the Wood-Saxon potential, of the pairing hamiltonian and of the multipole interaction used to calculate the properties of ${ }^{92,94} \mathrm{Zr}$ and ${ }^{94} \mathrm{Mo}$ [Ber99, Pon14].

$C_{j m j^{\prime} m^{\prime}}^{\lambda \mu}$ is a Clebsch-Gordan coefficient. The pairing force tends to couple nucleons (note that proton-neutron pairing is neglected) to spin-zero pairs motivating the following ansatz for the ground state

$$
|B C S\rangle \sim \prod_{k>0}\left(\alpha_{\bar{k}} \alpha_{k}\right)|0\rangle
$$

where the so called quasiparticle states $\alpha_{k}^{+}$are defined by the Bogoliubov transformation

$$
\begin{aligned}
& \alpha_{k}^{+}:=u_{k} \mathbf{a}_{k}^{+}-v_{k} \mathbf{a}_{\bar{k}} \\
& \alpha_{\bar{k}}^{+}:=u_{k} \mathbf{a}_{\bar{k}}^{+}-v_{k} \mathbf{a}_{k} .
\end{aligned}
$$

$k$ labels a state with quantum numbers $k=(n, j, l, m)$ and $\bar{k}$ stands for its time reversed counterpart i.e. $\bar{k}=(n, j, l,-m)$. In Eq. $5.23 k$ runs over all single-particle states, $|0\rangle$ is the vacuum state and the square of the coefficients $u_{k}$ and $v_{k}$ are the probabilities that the state $k$ is either empty or occupied. The strength of the pairing force $G_{\tau}^{(0)}$ is determined by a fit to odd-even mass differences in the mass region of interest resulting in the values given in Tab. 5.1. The ansatz of Eq. 5.23 is adopted from the BCS theory of superfluidity in solid state physics developed by Bardeen, Cooper and Schrieffer [Bar57]. The coefficients $u_{k}$ and $v_{k}$ can be determined deploying the variational principle under the constraint of a distinct particle number $N$

$$
\delta\left\{\left\langle B C S\left|\mathbf{H}_{s p}+\mathbf{H}_{\text {pair }}\right| B C S\right\rangle-\lambda\langle B C S|\mathbf{N}| B C S\rangle\right\}=0 .
$$

$\lambda$ is a Lagrange multiplicator (also called Fermi surface) and can be interpreted as the energy which is on average necessary to add an additional particle. The effect of the pairing force is evident from Fig. 5.3. Pairs of nucleons are scattered over different $j$-shells leading to a nonzero occupation probability above the Fermi surface. The BCS ground state can be considered as the quasiparticle vacuum and in even-even nuclei one can define - in analogy to the particlehole spectra of the extreme shell model - two-quasiparticle excitation spectra relative to the BSC ground state

$$
|\phi\rangle=\alpha_{k 1}^{+} \alpha_{k 2}^{+}|B C S\rangle \text {. }
$$

These spectra are shown for the case of ${ }^{92} \mathrm{Zr}$ in Fig. 5.4. 

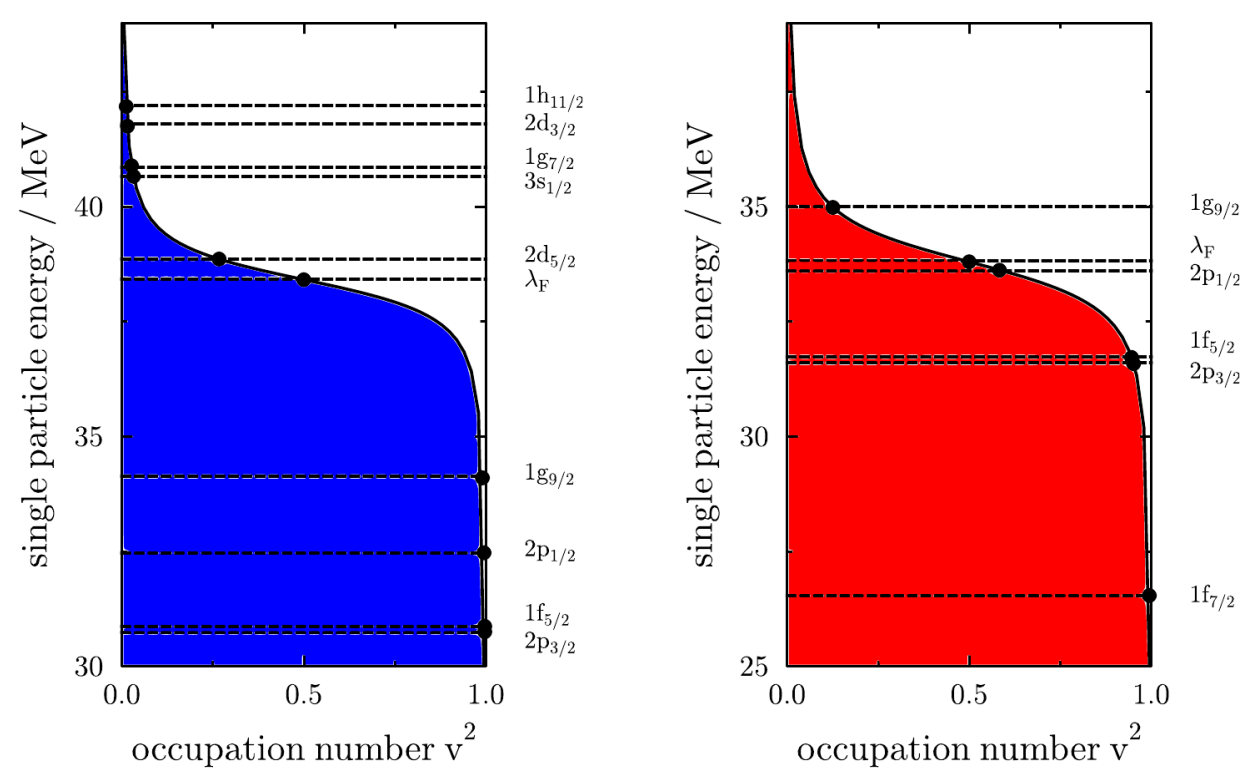

Figure 5.3: The single particle spectra and the occupation numbers of ${ }^{92} \mathrm{Zr}$ for neutrons (lefthand side) and protons (right-hand side).
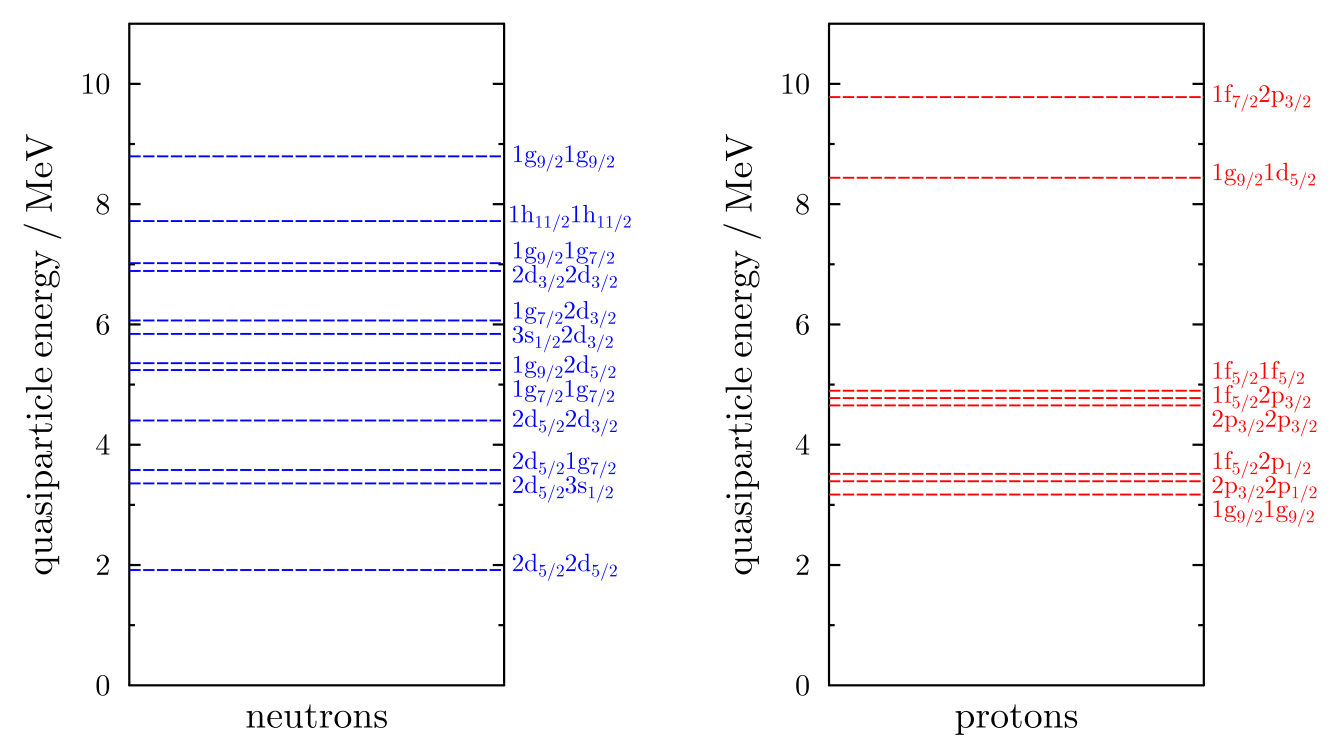

Figure 5.4: The two-quasiparticle spectra of ${ }^{92} \mathrm{Zr}$ for neutrons (left-hand side) and protons (righthand side).

In the next step the $\mathbf{H}_{\mathrm{m}}^{\mathrm{ph}}$ term of Eq. 5.19 is included in the diagonalization procedure being responsible for the mixing of two-quasiparticle states. The full QPM hamiltonian can be rewritten in the quasiparticle basis as [Ber99]

$$
\mathbf{H}_{q p m}=\sum_{\tau}^{n, p} \sum_{j, m} \epsilon_{j} \alpha_{j m}^{+} \alpha_{j m}+\sum_{\lambda \mu} \sum_{\tau \rho}^{ \pm 1}\left(\kappa_{0}^{(\lambda)}+\rho \kappa_{1}^{(\lambda)}\right) \mathbf{M}_{\lambda \mu}^{+}(\tau) \mathbf{M}_{\lambda \mu}^{+}(\rho \tau)
$$


with the multipole operators

$$
\begin{aligned}
& \mathbf{M}_{\lambda \mu}^{+}=\sum_{j j^{\prime}}^{\tau} \frac{f_{j j^{\prime}}^{(\lambda)}}{\sqrt{2 \lambda+1}}\left\{\frac{u_{j j^{\prime}}^{(+)}}{2}\left(\left[\alpha_{j}^{+} \alpha_{j^{\prime}}^{+}\right]_{\lambda \mu}+(-1)^{\lambda-\mu}\left[\alpha_{j^{\prime}}^{+} \alpha_{j}^{+}\right]_{\lambda-\mu}\right)-v_{j j^{\prime}}^{(-)} \mathbf{B}_{\tau}\left(j j^{\prime} ; \lambda \mu\right)\right\}, \\
& \mathbf{B}_{\tau}\left(j j^{\prime} ; \lambda \mu\right)=\sum_{m m^{\prime}}(-1)^{j^{\prime}+m^{\prime}} C_{j m j^{\prime} m^{\prime}}^{\lambda \mu} \alpha_{j m}^{+} \alpha_{j^{\prime}-m^{\prime}} \cdot
\end{aligned}
$$

The radial dependence of the residual interaction is contained in the reduced matrix element $f_{j j^{\prime}}^{\lambda}=\left\langle j\left\|i^{\lambda} f_{\lambda}^{\tau}(r) Y_{\lambda}(\Omega)\right\| j^{\prime}\right\rangle$ where $f_{\lambda}^{\tau}(r)$ is taken to be the first derivative of the Wood-Saxon potential of Eq. 5.20. The coefficients $u_{j j^{\prime}}^{( \pm)}$and $v_{j j^{\prime}}^{( \pm)}$are defined as $u_{j j^{\prime}}^{( \pm)}=u_{j} v_{j^{\prime}} \pm u_{j^{\prime}} v_{j}$ and $v_{j j^{\prime}}^{(\mp)}=u_{j} u_{j^{\prime}} \mp v_{j} v_{j^{\prime}}$.

In principle it is desirable to diagonalize the hamiltonian of Eq. 5.27 in the full quasiparticle basis. However this is not feasible due to the high dimension of the resulting matrix. In the so called Quasiparticle Random-Phase Approximation (QRPA) approach one truncates the large basis utilizing the following ansatz for the phonon operator which projects the ground state on the excited states [Ber99]:

$$
\mathbf{Q}_{\lambda \mu i}^{+}=\frac{1}{2} \sum_{\tau}^{n, p} \sum_{j j^{\prime}}\left\{\psi_{j j^{\prime}}^{\lambda i}\left[\alpha_{j}^{+} \alpha_{j^{\prime}}^{+}\right]_{\lambda \mu}-(-1)^{\lambda-\mu} \phi_{j j^{\prime}}^{\lambda i}\left[\alpha_{j^{\prime}} \alpha_{j}\right]_{\lambda-\mu}\right\}
$$

The so called forward $\left(\psi_{j j^{\prime}}^{\lambda i}\right)$ and backward amplitudes $\left(\phi_{j j^{\prime}}^{\lambda i}\right)$ can be determined from the QRPAequations which are obtained through a variational procedure

$$
\delta\left\{\left\langle\mathrm{QRPA}\left|\mathrm{H}_{q p m}\right| \mathrm{QRPA}\right\rangle-\left(\omega_{\lambda i} / 2\right)\left[\sum_{j j^{\prime}}\left\{\left(\psi_{j j^{\prime}}^{\lambda i}\right)^{2}-\left(\phi_{j j^{\prime}}^{\lambda i}\right)^{2}\right\}-2\right]\right\}=0
$$

where $|\mathrm{QRPA}\rangle$ is the QRPA-vacuum and $\omega_{\lambda i}$ is the energy of the $i$-th phonon with multipolarity $\lambda$. The forward and backward amplitudes fulfill the normalization condition

$$
\sum_{j j^{\prime}}\left[\psi_{j j^{\prime}}^{\lambda i} \psi_{j j^{\prime}}^{\lambda i^{\prime}}-\phi_{j j^{\prime}}^{\lambda i} \phi_{j j^{\prime}}^{\lambda^{\prime} i^{\prime}}\right]=2 \delta_{i i^{\prime}} \delta_{\lambda \lambda^{\prime}}
$$

The ratio of the strengths of the isoscalar to isovector residual interactions is kept constant to a value of $\kappa_{0}^{(\lambda)} / \kappa_{1}^{(\lambda)}=-1.2$ independent of the multipolarity or mass region. This value was determined to describe the properties of isovector resonances. The absolute strength is fixed to achieve a good description of the energy and the transition strength of the lowest state of each multipolarity. The corresponding values are given in Tab. 5.1.

Many nuclear structure calculations stop at this point and do not include the coupling between different phonon states. This makes it difficult to make a direct comparison to experiment, e.g. it is well known that the energies of the low-lying states are too high on the QRPA-level. The full QPM hamiltonian can be rewritten in terms of phonon operators as [Ber99]

$$
\mathbf{H}_{q p m}=\sum_{\lambda \mu i} \omega_{\lambda i} \mathbf{Q}_{\lambda \mu i}^{+} \mathbf{Q}_{\lambda \mu i}+\mathbf{H}_{i n t}
$$




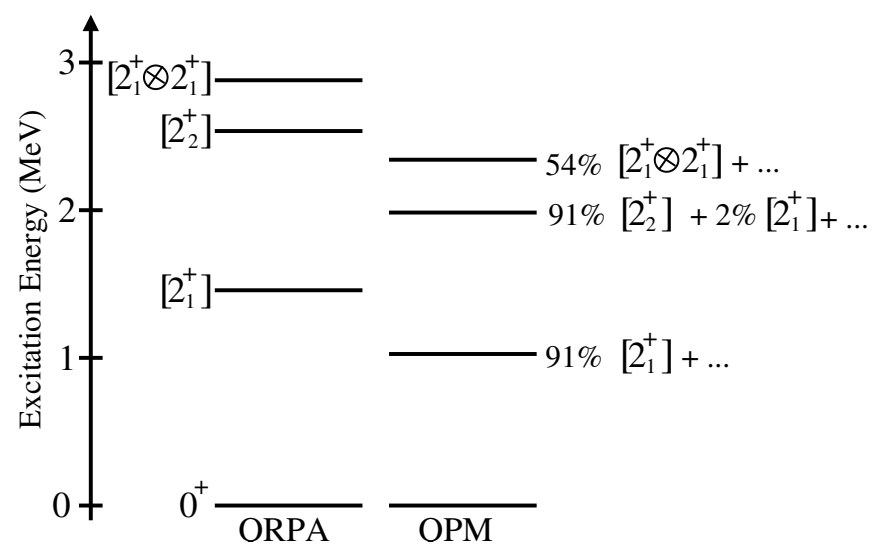

Figure 5.5: Comparison of the QRPA and QPM spectra of the lowest $2^{+}$states of ${ }^{92} \mathrm{Zr}$.

Clearly, the Hamiltonian is not diagonal in the phonon basis because of the term $\mathbf{H}_{\text {int }}$ which introduces a mixing of phonon states. In order to truncate the resulting matrix in the phonon basis to a feasible size the QPM includes only one, two - and three phonon states. In addition phonon states above $\sim 10 \mathrm{MeV}$ are neglected. The experience shows that both approximation are sufficient to account for the main properties of low-lying states. The matrix is diagonalized and the final QPM states are obtained. The effect of the coupling to more complex configurations can be seen in Fig. 5.5, e.g. the energy of the $2_{1}^{+}$state - which is too high on the QRPA-level (the experimental energy is $934 \mathrm{keV}$ ) - is lowered by the coupling to more complex configurations bringing it closer to the experimental value.

\subsubsection{Electromagnetic transition strengths and transition densities}

In order to perform a test of the QPM wave functions a comparison to electromagnetic transition strength and electron and proton scattering cross sections give valuable information on their validity. In the QPM the electric transition matrix element from a QRPA-phonon to the ground state can be written as

$$
\left\langle Q_{\lambda i}\|\mathscr{M}(E \lambda)\| 0_{g . s .}^{+}\right\rangle=\sum_{\tau}^{n, p} e_{\tau}^{(\lambda)} \sum_{j, j^{\prime}} \frac{u_{j j^{\prime}}^{(+)}}{2}\left\langle j\|E \lambda\| j^{\prime}\right\rangle\left(\psi_{j j^{\prime}}^{\lambda i}+\phi_{j j^{\prime}}^{\lambda i}\right) .
$$

$\left\langle j\|E \lambda\| j^{\prime}\right\rangle$ is the single-particle transition matrix element. Due to the large configuration space the QPM uses no effective charges i.e. $e_{n}^{(\lambda)}=0$ and $e_{p}^{(\lambda)}=1$. The form of Eq. 5.34 clear that the contribution of a two-quasiparticle to the transition matrix element is determined by its contribution to the wave function $\psi_{j j^{\prime}}^{\lambda \lambda}+\phi_{j j^{\prime}}^{\lambda i}$ and by the size of the corresponding single particle matrix element. For the discussion of mixed-symmetry states the M1-transition between QRPAphonons is in particular important. The magnetic transition matrix element is given through

$$
\begin{aligned}
& \left\langle Q_{\lambda_{3}}\left\|\mathscr{M}\left(M \lambda_{1}\right)\right\| Q_{\lambda_{2^{i}}{ }^{\prime}}\right\rangle=\sum_{\tau}^{p, n} \sum_{j_{1} j_{2} j_{3}} v_{j j^{\prime}}^{(+)} \cdot\left\langle j_{1}\left\|M \lambda_{1}\right\| j_{2}\right\rangle . \\
& \cdot\left\{\begin{array}{ccc}
\lambda_{3} & \lambda_{2} & \lambda_{1} \\
j_{1} & j_{2} & j_{3}
\end{array}\right\}\left(\psi_{j_{2} j_{3}}^{\lambda_{3}} \psi_{j_{3} j_{1}}^{\lambda_{2}}+\phi_{j_{2} j_{3}}^{\lambda_{3}} \phi_{j_{3} j_{1}}^{\lambda_{2}}\right) .
\end{aligned}
$$


The present work makes use of the transition densities which are the main input to calculate electron and proton scattering cross section. In the framework of the QPM the matter transition density of a pure QRPA-phonon is given by [Pon14]:

$$
\rho_{i}^{J}(r)=\sum_{j j^{\prime}}^{N, Z} \frac{u_{j j^{\prime}}^{(+)}}{2} \rho_{j j^{\prime}}^{J}(r) \cdot\left(\psi_{j j^{\prime}}^{J i}+\phi_{j j^{\prime}}^{J i}\right)
$$

where

$$
\rho_{j j^{\prime}}^{J}(r)=(-1)^{j-\frac{1}{2}} i^{l^{\prime}-l-J} \frac{\sqrt{2 j+1} \sqrt{2 j^{\prime}+1}}{4 \sqrt{2 J+1} \sqrt{\pi}}\left(1+(-1)^{l+l^{\prime}+J}\right)\left\langle j \frac{1}{2} j^{\prime} \frac{-1}{2} \mid J 0\right\rangle p_{j}^{*}(r) p_{j^{\prime}}(r) .
$$

The functions $\mathrm{p}_{j}(\mathrm{r})$ are the radial parts of the single-particle wave functions of the Wood-Saxon potential defined in Eq. 5.20. In dependence of the contributing two-quasiparticle states the transition densities of the various QRPA-phonons can differ significantly resulting in different electron and proton scattering cross sections and providing an excellent test ground for the QPM wave functions.

\subsubsection{Definition of a mixed-symmetry state in the framework of the QPM}

The definition of a mixed-symmetry state is given in Sect. 5.2 in the framework of the IBM2. In the present thesis all theoretical calculations have been done in the QPM which is in contrast to the IBM-2 a microscopic approach. Therefore one has to find a definition of what exactly is a mixed-symmetry state in the framework of the QPM. One way would be to map the wave functions of the QPM on the boson wave functions of the IBM-2 utilizing the OAI mapping [Ots78, Nak91]. The OAI mapping gives a formal relationship between both models and one would be able to determine quantitatively the overlap of the QPM wave functions with the wave function of a mixed-symmetry state in the IBM- 2 .

However in the present work a more loose connection is used. In the investigated nuclei - ${ }^{92,94} \mathrm{Zr}$ and ${ }^{94} \mathrm{Mo}$ - one finds the situation that the lowest two quadrupole QRPA-phonons are dominated by the $\left(2 d_{5 / 2} \otimes 2 d_{5 / 2}\right)_{\mathrm{n}}$ - and $\left(1 g_{9 / 2} \otimes 1 g_{9 / 2}\right)_{\mathrm{p}}$-two-quasiparticle states being in-phase in one phonon and out-of-phase in an other one. In the following the phonon where both states are in-phase is considered as the microscopic counterpart of the fully symmetric one-phonon state, while the phonon where both states are out-of-phase is the counterpart of the mixed-symmetry state. The justification for this relationship is firstly given by the similar physical observables. As discussed in Sect. 5.2 the experimental signatures of a quadrupole mixed-symmetry state in the IBM-2 are a large M1-transition to the $2_{\text {fss }}^{+}$state of the order of $\sim 1 \mu_{\mathrm{N}}^{2}$ and weakly collective E2-transition to the ground state. Both signatures are also fulfilled by the corresponding QRPAphonon. Secondly one can see a similarity in the structure of the wave function given in Fig. 5.2 if one considers the $\left(2 d_{5 / 2} \otimes 2 d_{5 / 2}\right)_{\mathrm{n}}$ - and $\left(1 g_{9 / 2} \otimes 1 g_{9 / 2}\right)_{\mathrm{p}}$-two-quasiparticle states as $d_{n}^{+}$and $d_{p}^{+}$ states respectively. 


\section{The experimental setup at iThemba LABS}

The five proton scattering experiments analyzed in this thesis were carried out at the iThemba Laboratory for Accelerator Based Sciences (iThemba LABS) located near Somerset West in South Africa. Their purpose is to measure the cross sections of low-lying states ( $\lesssim 3 \mathrm{MeV}$ ) of ${ }^{92,94} \mathrm{Zr}$, ${ }^{94,96} \mathrm{Mo}$ and ${ }^{70} \mathrm{Zn}$ and in particular that of the $2_{1}^{+}$and $2_{\mathrm{ms}}^{+}$states. The aim of this chapter is to outline the experimental setup at iThemba LABS. In the beginning an overview about the facility is given (Sect. 6.1). Then the magnetic spectrometer used to momentum analyze the scattered protons is described (Sect. 6.2). Afterward the detector system - consisting of two vertical drift chambers (VDC) and two plastic scintillators - on the focal plane of the magnetic spectrometer is discussed in more detail (Sect. 6.3). In Sect. 6.4 the electronic setup and the data acquisition system (DAQ) is explained. Finally in Sect. 6.5 details on the conducted experiments are given regarding targets, covered angles, energy resolution and other things.

The experiments on ${ }^{92} \mathrm{Zr}$ and ${ }^{94} \mathrm{Mo}$ - which were conducted in 2005 - used an older, slightly different setup than the one discussed in this chapter, which is valid for the ${ }^{94} \operatorname{Zr}\left(p, p^{\prime}\right),{ }^{96} \operatorname{Mo}\left(p, p^{\prime}\right)$ and ${ }^{70} \mathrm{Zn}\left(p, p^{\prime}\right)$ experiments. Since the old setup is extensively discussed in Ref. [Wal10], it is not further considered in this chapter.

\subsection{The iThemba Laboratory}

The iThemba Laboratory is a multi-disciplinary research facility [iTH] covering a wide range of applications. Its core activities are basic research using particle beams, proton and neutron therapy for cancer treatment and the production of radioactive isotopes for nuclear medicine.

The floor plan of the laboratory is presented in Fig. 6.1. The particle beams are produced with one of the three ion sources presently available, namely the Electron Cyclotron Resonance (ECR) ion source, the polarized ion source and the Penning Ionization Gauge (PIG). In the present experiment the PIG source was used and the protons were pre-accelerated by the Solid Pole Cyclotron 1 (SPC1) and injected in the Separated Sector Cyclotron. Here the protons are further accelerated to the desired energy. To minimize the contributions of two-step processes to the reaction mechanism the maximum available energy of $200 \mathrm{MeV}$ was chosen for this experiment. Afterward the particle beam is transferred to the experimental setup of choice like the gamma detector array AFRODITE [AFR] or the K600 magnetic spectrometer [Nev11]. The latter was used in the present experiment and is described in detail in the next section.

\subsection{The K600 magnetic spectrometer}

The protons were momentum analyzed with the K600 QQD magnetic spectrometer which is displayed in Fig. 6.2. The protons hit the target of interest and a part of them is scattered in the solid angle covered by the spectrometer. The solid angle acceptance amounts to $3.48 \mathrm{msr}$ and is defined by the collimator with a diameter of $49 \mathrm{~mm}$ located upstream of the target position. The two dipoles of the K600 are needed to achieve dispersion of the scattered protons, while 


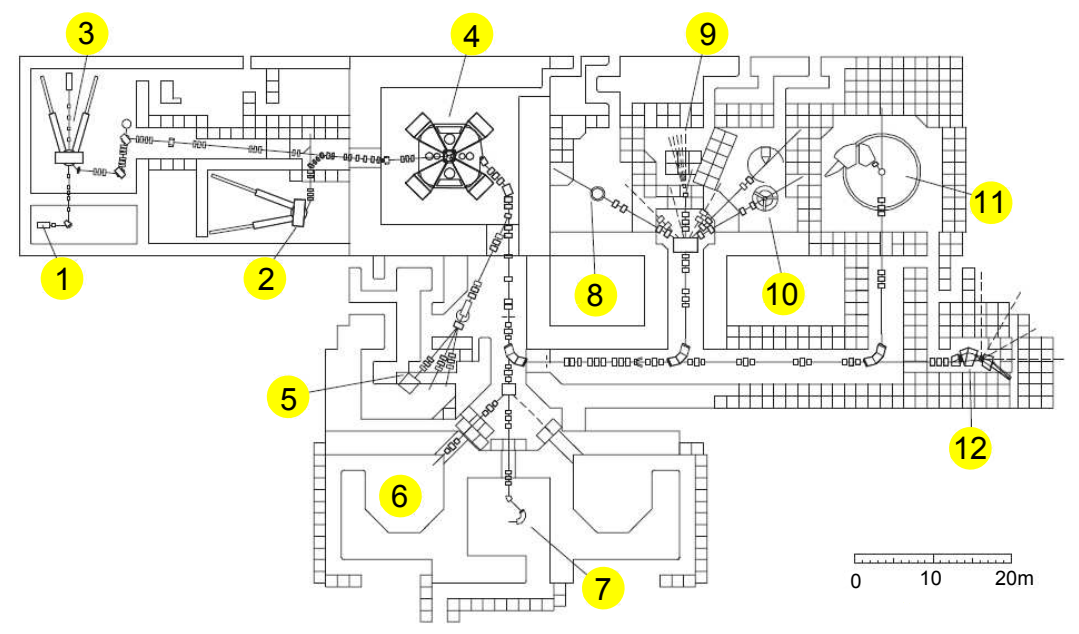

Figure 6.1: Floor plan of the iThemba LABS facility in South Africa with its main experimental setups: (1) ECR ion source, (2) SPC1 injector cyclotron and PIG source, (3) SPC2 injector cyclotron and polarized source, (4) separated-sector cyclotron, (5) radioactive isotope production, (6) proton therapy, (7) neutron therapy, (8) experiments with charged particles, (9) experiments with neutron beams, 10 -spectroscopy, 11 K600 spectrometer and (12) beam swinger magnet (taken, and slightly changed, from Refs. [New96, Wal10]).

the quadrupole magnet is used for vertical focusing. In addition two trim coils - situated inside the dipole magnets - help to correct the first and second order aberrations of the spectrometer meaning the dependence of the momentum of the protons on the scattering angle. These dependencies can be further corrected offline.

The covered momentum range is given by $\Delta p=p_{0} \cdot \frac{x}{D}$, where $p_{0}$ is a reference momentum, $x$ the length of the focal plane and $D$ is the dispersion [Nev01]. Three different dispersion modes are available at the K600: low, medium and high with dispersion values of $6.2 \mathrm{~cm} / \%, 8.4$ $\mathrm{cm} / \%$ and $9.8 \mathrm{~cm} / \%$. In the present experiment the medium dispersion mode was chosen. The magnetic fields of the magnets were set in such a way that the elastic peak was as far as possible on the high momentum side of the focal plane to fully exploit the momentum acceptance.

In order to compensate for the energy spread of the beam - which would result in a worse spatial resolution on the focal plane - the spectrometer is operated in dispersion matching mode. The beam is slightly defocused on the target in such a way that particles - which have different momenta due to the energy spread of the beam but leave the target nucleus in the same nuclear state, travel different paths through the spectrometer and are still focused on the same horizontal position on the focal plane. The design value of the resolution of the K600 spectrometer for a dispersion matched beam is $\delta p / p=10^{-4}$.

\subsection{The focal plane detector package}

In order to measure the momentum of the particle the position of its crossing point in the dispersive direction (referred to as $X$ ) on the focal plane has to be determined. In the present 


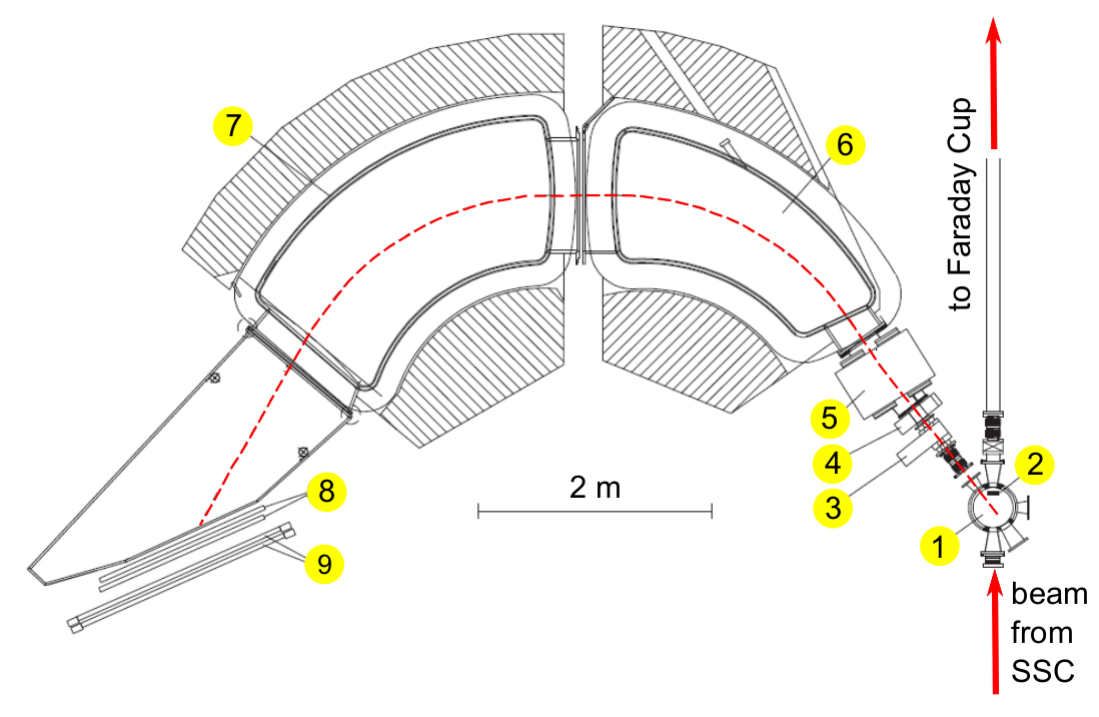

Figure 6.2: The K600 spectrometer at iThemba LABS: (1) Scattering chamber, (2) internal Faraday cup for small-angle scattering experiments, (3) set of collimators, (4) sextupole magnet, (5) quadrupole magnet, (6) first dipole magnet, (7) second dipole magnet, $(8)$ one $X$ - and one $X U$-chamber, and (9) two plastic scintillators (taken, and slightly changed, from Refs. [New96, Wal10]).

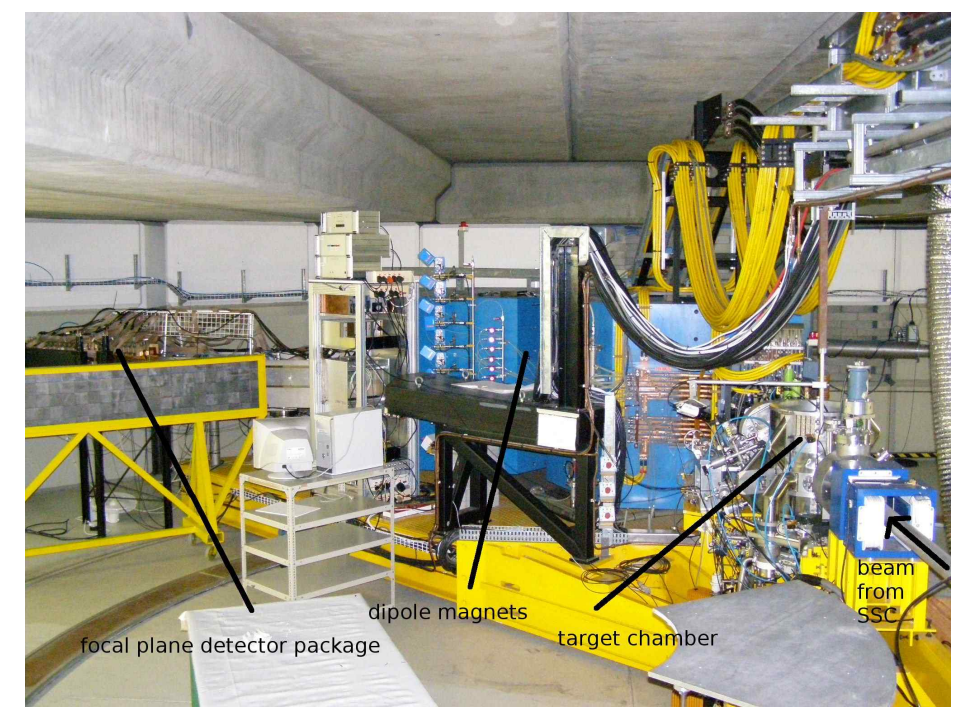

Figure 6.3: Photograph of the $\mathrm{K} 600$ spectrometer including the target chamber, the dipole magnets and the focal plane detector package. The lead wall in front of the focal plane is to protects the detectors from background gamma rays coming from the direction of the target chamber.

experiment this is achieved by using a focal plane detector package consisting of two vertical drift chambers (VDC) - which are positioned in such a way that its plane coincide to first order with the focal plane of the magnetic spectrometer [Nev01] - and two plastic scintillators. The VDCs are an excellent tool to obtain precisely the position information perpendicular to its wires. Figure 6.4 presents a cut-out illustration of the so called XU-VDC. It consists of two wire 
planes, one to determine the $X$-coordinate and one to determine the $U$-coordinate. The $U$-axis is rotated by $50^{\circ}$ with respect to the $X$-axis. The second VDC - also of type $X$ - is positioned 281 mm upstream of the $X U$-VDC. The three coordinates $X_{1}, X_{2}$ and $U_{2}$ allow the determination of the $X$ - and $Y$-coordinates and also the angle $\theta_{\mathrm{FP}}$ of the particle track with respect to the $X$-axis.

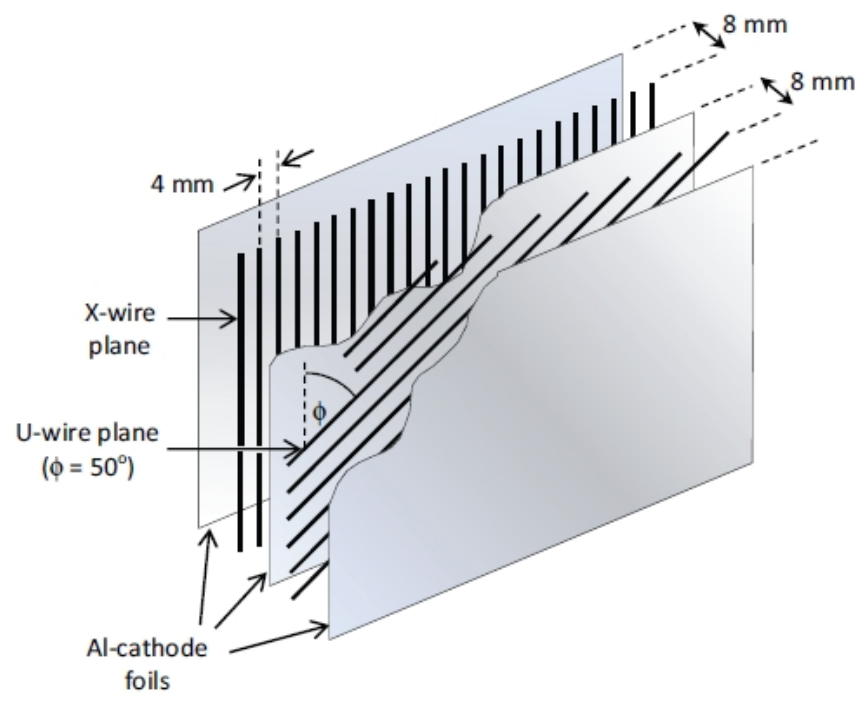

Figure 6.4: Cut-out illustration of the $U X$-VDC used in the experiment. The wires of the $U$-plane are rotated by $50^{\circ}$ degrees with respect to the wires of the $X$-plane. This allows the determination of the $Y$-coordinate of the crossing point (taken from [Zyl12]).

The two plastic scintillators positioned down-stream of the VDCs provide a time reference for the VDCs. Furthermore they allow a time-of-flight measurement and give a $\Delta E-\Delta E$ information for particle identification.

\subsection{Electronic setup and data acquisition}

The experiment is done using standard NIM and VME electronics. The main VME modules are one V792 QDC and seven V1190 TDCs from the company Caen. The large number of TDCs is necessary to provide a TDC channel for each VDC channel. The VME modules and the data flow are controlled by the MIDAS data acquisition software [MID07].

Figure 6.5 gives an overview about the electronic setup of the experiment. Both plastic scintillators - denoted in Fig. 6.5 as paddle 1 and 2 - were read out at both ends and the corresponding signals were amplified and split by a linear fan out. One of the outputs of each linear fan out was fed in a QDC to provide a $\Delta E$ information for particle identification. The other output was processed with a CFD and a mean timer to provide a time reference for the VDCs which is independent of the interaction point. Coincidences between both paddles are selected using a four fold logic unit which serves as a AND-gate.

This coincidence signal was then fed in two different branches. In the first it serves to select RF signals which are in coincidence with a particle which deposited energy in both plastic scintillators. The RF signal starts the time measurement of the TDCs to provide the TOF measurement for particle identification. Since the whole setup operates in common stop mode also all VDC signals provide start signals for the TDCs. In the second branch the paddle-coincidence signal is again split. One of the two signal provides the QDC gate for the energy measurement of the 
paddle signals and the other one is delayed by a gate and delay generator and serves as the common stop signal for the TDCs. The time difference between this signal and the corresponding VDC or RF signal gives the required time information.

For determining absolute cross sections it is required to measure the total charge collected at the beam dump. This is done by sending the current at the beam dump to a current integrator module (Brookhaven Intruments Corporation Model 1000C) which gives a rate which is proportional to the measured charge (more details on the current integrator (CI) are given in Sect. 7.7). The rate is recorded with a scaler module. To measure the dead time the signal from the CI is fed in another scaler which is inhibited with the DAQ busy signal as shown in Fig. 6.5. The ratio of both scaler values allows to determine the dead time. It should be noted that the DAQ busy signal is actually the QDC busy, i.e. the time the QDC needs to measure the charge of one signal. All other components i.e. the TDCs, the NIM electronics and the readout of the VME modules are assumed to be dead time free.

The data words containing the information on the event are readout from the memories of the TDCs, QDC and Scalers via the VME backplane and are further processed with the MIDAS software and saved on hard disk. The unpacking and analysis of the raw data words is done offline with a program developed at iThemba LABS, as discussed in chapter 7.

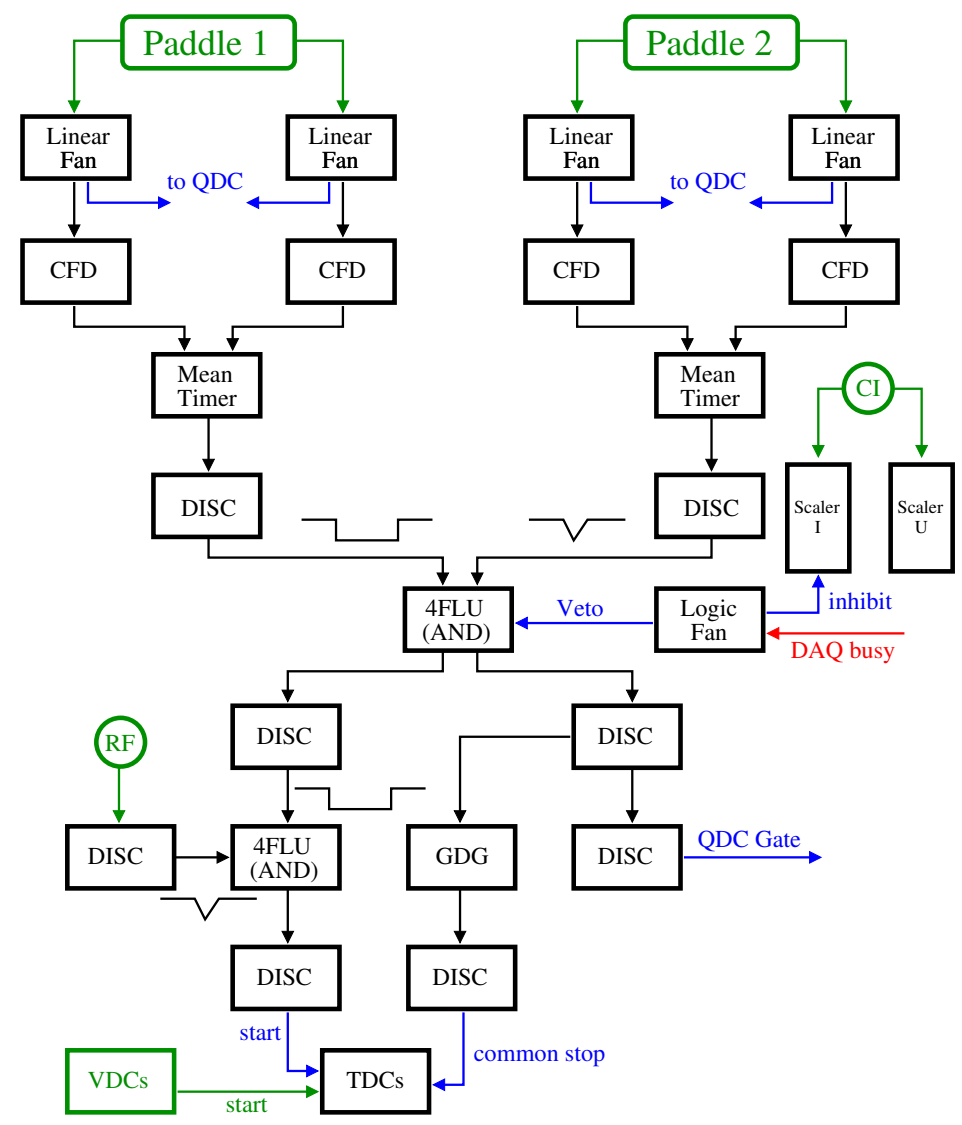

Figure 6.5: Simplified block diagram of the electronics of the experiment. Details are given in the text. The abbreviations are the following: Linear Fan = Linear Fan in/out, CFD $=$ Constant Fraction Discriminator, DISC $=$ Discriminator, 4FLU $=4$ Fould Logic Unit, Logic Fan = Logic Fan in/out, GDG = Gate Delay Generator, Scaler I = inhibited Scaler, Scaler $\mathrm{U}=$ uninhibited Scaler, $\mathrm{Cl}=$ Current Integrator, RF = Radio Frequency Signal, TDC $=$ Time to Digital Converter and QDC = Charge to Digital Converter. 


\subsection{Conducted experiments}

The experiments investigating the nuclei ${ }^{94} \mathrm{Zr}$, ${ }^{96} \mathrm{Mo}$ and ${ }^{70} \mathrm{Zn}$ were conducted on three weekends in March 2011 at the iThemba laboratory. The beam time was limited to the weekends because the facility is used for production of radioactive isotopes and cancer treatment from Monday till Friday. The spectrometer and electronics were setup as described in the last sections. For all three experiments the PIG ion source and the SPC1 cyclotron to pre-accelerate the beam were used. The final beam energy was chosen to be $200 \mathrm{MeV}$.

In the beginning of each weekend the first task was a sieve slit measurement to calibrate the scattering angle. Next the spectrometer angle were set to the desired value. After checking the beam quality with an empty frame run the energy calibration was performed using a ${ }^{24} \mathrm{Mg}$ target. Since the beam conditions slightly change over time - resulting in a shift of the horizontal focal plane position of the excitation spectrum - it was repeated approximately every hour. Due to the limited time no calibration measurements were taken for ${ }^{96}$ Mo on the last weekend. This is in principle not required since the focus of the present experiment are the low-lying states, i.e. $E_{x}<3 \mathrm{MeV}$. This energy region can be reliably calibrated with already known excited states of the investigated nucleus.

To collect as much data as possible the beam current was typically at the maximum value feasible for the experimental setup. The limiting factor was the dead time of the DAQ. It was decided to keep the dead time at a value $<5 \%$. Depending on the scattering angle and the target this results in beam currents of $0.6-24.0 \mathrm{nA}$ as shown in Tab. 6.1. The measurement time for each angle was chosen to collect sufficient statistics for the $2_{1}^{+}$and $2_{\mathrm{ms}}^{+}$states and also for the higher-lying $2^{+}$states. Hence the measurement time strongly differed for various angle settings since the $2^{+}$proton scattering cross section varies by one order of magnitude in the investigated angular range. The scattering angle was changed in steps of $2^{\circ}$ or $2.5^{\circ}$ degrees in a range from $8^{\circ}$ to $25^{\circ}$ degrees as indicated in Tab. 6.1.

\begin{tabular}{|c|c|c|c|}
\hline & $\overline{94} \mathrm{Zr}$ & ${ }^{96} \mathrm{Mo}$ & ${ }^{70} \mathrm{Zn}$ \\
\hline areal density of target & $2.93 \mathrm{mg} / \mathrm{cm}^{2}$ & $2.98 \mathrm{mg} / \mathrm{cm}^{2}$ & $4.9 \mathrm{mg} / \mathrm{cm}^{2}$ \\
\hline isotopic composition & ${ }^{96} \operatorname{Zr~} 0.24 \%$ & ${ }^{92}$ Mo $0.18 \%$ & ${ }^{70}$ Zn $95.42 \%$ \\
\hline & ${ }^{94} \mathrm{Zr} 96.07 \%$ & ${ }^{94}$ Mo $0.20 \%$ & ${ }^{68} \mathrm{Zn} 4.45 \%$ \\
\hline & ${ }^{92} \mathrm{Zr} 0.92 \%$ & ${ }^{95}$ Mo $0.93 \%$ & ${ }^{67} \mathrm{Zn} 0.13 \%$ \\
\hline & ${ }^{91} \mathrm{Zr} 0.69 \%$ & ${ }^{96}$ Mo $96.69 \%$ & ${ }^{66} \mathrm{Zn}<0.02 \%$ \\
\hline & ${ }^{90} \mathrm{Zr} 2.08 \%$ & ${ }^{97}$ Mo $0.99 \%$ & ${ }^{64} \mathrm{Zn}<0.02 \%$ \\
\hline & & ${ }^{98}$ Mo $0.89 \%$ & \\
\hline & & ${ }^{100} \mathrm{Mo} 0.16 \%$ & \\
\hline energy resolution & $50-78 \mathrm{keV}$ & $29-43 \mathrm{keV}$ & $32-52 \mathrm{keV}$ \\
\hline beam energy & $200 \mathrm{MeV}$ & $200 \mathrm{MeV}$ & $200 \mathrm{MeV}$ \\
\hline beam current & $1.0-24.0 \mathrm{nA}$ & $0.4-6 \mathrm{nA}$ & $0.6-9.5 \mathrm{nA}$ \\
\hline measured angles & $\begin{array}{c}8.05,9.90,12.45,14.90 \\
17.45,19.90,22.40,24.95\end{array}$ & $\begin{array}{c}7.95,9.95,12.45,14.95 \\
17.45,19.95,22.45\end{array}$ & $\begin{array}{l}8.00,10.05,12.55,14.95, \\
17.45 .20 .00 .22 .45 .24 .95\end{array}$ \\
\hline evaluated energy range & $0-4.02 \mathrm{MeV}$ & $0-3.60 \mathrm{MeV}$ & $0-4.72 \mathrm{MeV}$ \\
\hline
\end{tabular}

Table 6.1: Data taking conditions and targets of the ${ }^{94} \mathrm{Zr}\left(p, p^{\prime}\right),{ }^{96} \mathrm{Mo}\left(p, p^{\prime}\right)$ and ${ }^{70} \mathrm{Zn}\left(p, p^{\prime}\right)$ experiments.

The achieved energy resolution (FWHM) was between $50-78 \mathrm{keV}$ for ${ }^{94} \mathrm{Zr}, 29-43 \mathrm{keV}$ for ${ }^{96} \mathrm{Mo}$ and $32-52 \mathrm{keV}$ for ${ }^{70} \mathrm{Zn}$. The worse energy resolution for ${ }^{94} \mathrm{Zr}$ was due to problems with the proton beam which could not be resolved during the first weekend of the beam time. For this reason only a relatively small number of excited states of ${ }^{94} \mathrm{Zr}$ could be analyzed. 
As presented in Tab. 6.1 all three targets were enriched to a percentage of $>95 \%$. As a matter of fact the contributions of other isotopes to the excitation spectra were small and only seen in case of the $2_{1}^{+}$and $3_{1}^{-}$states. The target thicknesses were small enough so that the contribution to the energy resolution due to multiple scattering processes in the target is small. 


\section{Data analysis and results of the proton scattering experiments}

In order to extract the absolute cross section of each state from the measured raw data a series of analysis steps have to be taken. First of all, the scattered protons are identified using the $\Delta E$ information of the two plastic scintillators on the focal plane and a time-of-flight (TOF) measurement (Sect. 7.1). Secondly, the position and angle information of the protons passing through the focal plane are determined using the three vertical drift chambers (VDCs). This involves setting up a suitable lookup table to convert drift times to drift distances (Sects. 7.2.2 and 7.2.3), a definition of what is considered as a valid event, an algorithm to determine the position from the drift distances and finally to calculate the horizontal and vertical positions and the horizontal scattering angle on the focal plane (Sect. 7.2). Before calibrating the horizontal position - which is related to the momentum of the proton - it is necessary to calibrate the horizontal scattering angle at the target position using the sieve slit data (Sect. 7.3) and to correct for the aberrations of the spectrometer and the kinematic dependence of the proton momentum on the scattering angle (Sect. 7.4). The energy calibration procedure is outlined in Sect. 7.5. Finally the absolute cross sections are determined (Sect. 7.7).

\subsection{Particle identification}

When hitting the target nuclei with a particle beam of high energy many reaction channels are open and a variety of different particles are produced. In order to conduct an accurate experiment with low background conditions, it is necessary to separate the different particle types. This is especially important if the cross section of the reaction of interest is small. A magnetic spectrometer alone is not capable of performing this task. For a particle of momentum $p$ and charge $q$ traveling in a constant magnetic field $B$ perpendicular to the momentum direction the following equation holds

$$
r B=\frac{p}{q}=R,
$$

with $r$ being the curvature radius, and $R$ the magnetic rigidity of the particle. Hence for a given magnetic field setting particles with similar magnetic rigidity follow similar trajectories and are not distinguishable from each other using their position on the focal.

Two different techniques are applied for particle identification at the K600. In the first method the radio-frequency signal of the SSC and the signal of the two plastic scintillators on the focal plane are used for a TOF measurement. Although the particles have similar magnetic rigidities their masses are different and hence their flight times $t$ from the target to the focal plane differ

$$
t=\frac{d}{v}=\frac{d}{c \sqrt{1-\left(\frac{m_{0} c^{2}}{E_{k i n}+m_{0} c^{2}}\right)^{2}}}
$$


with $v$ being the velocity, $d$ the traveled distance, $E_{\text {kin }}$ the kinetic energy and $m_{0}$ the rest mass. The ratio of the distance and the time resolution of the setup is sufficiently good to achieve a clean separation of light ions namely protons, deuterons, ${ }^{3} \mathrm{He}$ and ${ }^{4} \mathrm{He}$ by choosing an appropriate time window [New96]. The second method utilizes the $\Delta E$ information of the two
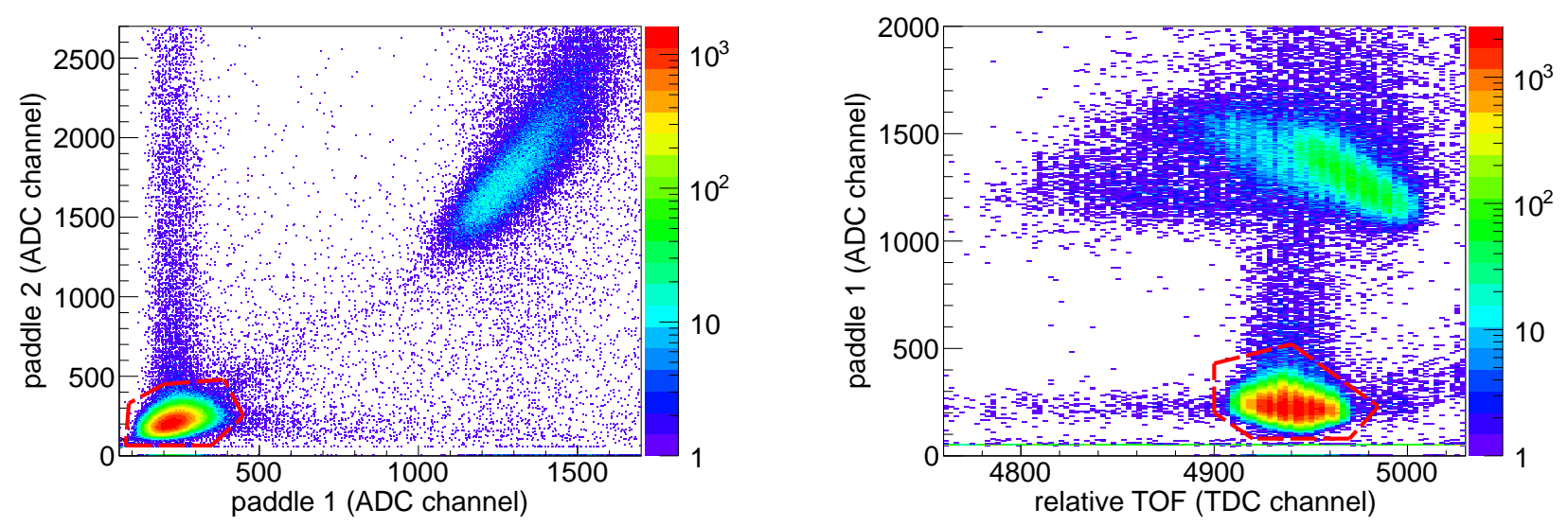

Figure 7.1: Examples of $\Delta E_{1}-\Delta E_{2}$ (left-hand side) and $\Delta E_{1}$-TOF (right-hand side) spectra. The used software gates on elastic and inelastic scattered protons are marked in red. The origin of the bump at $\Delta E \approx 1500$ is not understood.

plastic scintillators on the focal plane. According to the Bethe-Bloch formula [Kno10] a charged particle deposits per unit length (in the leading order) an energy of

$$
-\frac{d E}{d x} \sim \frac{q^{2}}{v^{2}}
$$

in the material. Hence each particle with a characteristic charge to velocity ratio deposits a specific amount of energies $\Delta E_{1,2}$ in the two plastic scintillators. In order to determine this quantity independently of the interaction point each plastic scintillator is readout at both ends and the product of both photo multiplier signals $P$ is taken $\Delta E \sim P($ left $) \cdot P($ right $)$.

Figure 7.1 displays examples of $\Delta E_{1}-\Delta E_{2}$ (left-hand side) and $\Delta E_{1}$-TOF (right-hand side) spectra. Software gates were set on suitable TOF and $\Delta E$ regions. This method of background suppression, however, is for the present experiment of secondary importance, since elastic and inelastic proton scattering dominate all other reaction channels. Nevertheless, a slight improvement of the peak to background ratio was achieved, which is useful for weakly excited states.

\subsection{Determination of the focal plane coordinates}

The main task of the focal plane detector system is to determine the horizontal coordinate $X$ of the intersection point (i.e. the coordinate in the dispersive direction) which is closely related to the momentum of the particle. Furthermore it is useful to measure the $Y$-coordinate (nondispersive direction) and the angle $\theta_{F P}$ of the trajaectory relative to the $X$-direction in order to obtain optimal energy resolution, background conditions and to be able to divide the solid angle of the spectrometer into sub ranges. All three quantities can be determined using the three VDCs $X_{1}, X_{2}$ and $U_{2}$. 


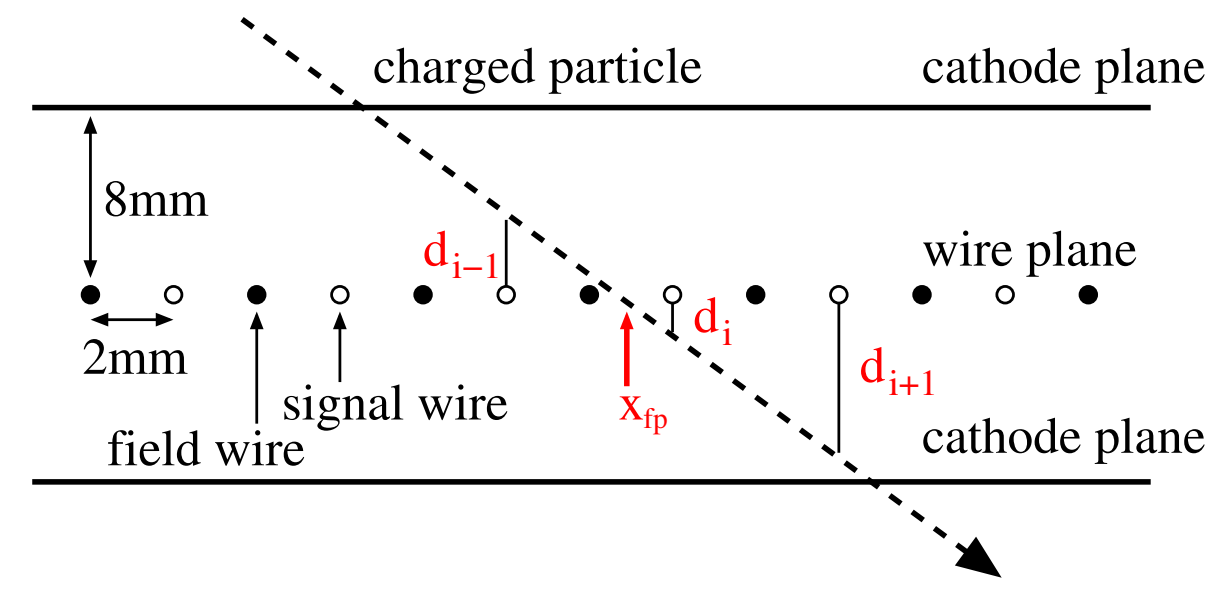

Figure 7.2: Schematic of a vertical drift chamber. The focal plane position $\mathrm{x}_{\mathrm{f} p}$ is calculated using the measured drift distances $d_{i-1}, d_{i}$ and $d_{i+1}$.

7.2.1 Working principle of the VDCs

A schematic of a VDC is shown in Fig. 7.2. Each VDC consists of two cathode planes and one wire plane in between. The field wires, which are located between each pair of signal wires, provide field shaping and prevent cross talk. The charged particles passing through the active volume of the VDC collide with the atoms of the working gas and create electron-ion pairs along its track. The electrons drift in the electric field - which is constant except very close to the signal wire - to the signal wires inducing a voltage pulse. The plastic scintillators - located behind the drift chambers - provide a reference time signal to determine the drift times which are characteristic of the track positions $d_{j}$ in the VDC. Due to the constant electric field - which results in a constant drift time - this relationship is expected to be linear. The setup of a suitable lookup table to convert drift times to drift distances is explained later in this section. Through proper positioning of the VDCs it is assured, that the scattered particles cross the focal plane at an angle of $\sim 35^{\circ}$ and hence cause on average several wire hits. Eventually the intersection point and the angle are determined by linear regression using the measured drift distances $d_{0} \ldots d_{n}$.

Not every event exhibit a physical meaningful distribution of wire hits along the wire plane. An event is considered as valid, i.e. included in the further analysis, if the following conditions are fulfilled. The number of wire hits has to be larger than 2 and smaller than 9. No double hits are allowed - meaning a wire is not allowed to fire more than once per event. The drift times are expected to have only one global minimum and no additional local minima. Furthermore in the region defined by the wires, which are farthest to the left and farthest to the right, there shall be only to 2 wires which have not fired.

It is possible to recover a part of the events which initially do not fulfill the conditions defined above using suitable algorithms, which are based on experience in operating a VDC. 
After determination of the three intersection points $X_{1}, X_{2}$ and $U_{2}$ of the three wire planes the final track of the particle can be calculated. The $X$-coordinate is directly taken to be the $X_{1}$. The following equations hold for $Y$ and $\theta_{F P}$,

$$
\begin{gathered}
X=X_{1}, \\
\theta_{F P}=\arctan \left(\frac{z_{\text {offset }}}{X_{2}+x_{\text {offset }}-X_{1}}\right), \\
Y=\frac{U_{2} \cdot \tan \theta_{F P}-16 \cdot \sin \theta_{\mathrm{U}}}{\sin \theta_{\mathrm{U}} \cdot \tan \theta_{F P}} \cdot \tan \theta_{\mathrm{U}}-X_{2},
\end{gathered}
$$

$x_{\text {offset }}$ and $z_{\text {offset }}$ are the offsets of the $X_{1}$ and $X_{2}$ wire planes in $x$ - and z-direction, respectively. $\theta_{U}$ is the angle between the wires of the $U_{2}$ - and $X_{1,2}$-wire planes.

\subsubsection{Conversion of drift times to drift distances}

In order to determine precisely the interception point of the charged particle with the VDC a lookup table is required to convert the measured relative drift times to drift distances. In the present experiment this is done using the 'integral time spectra' - method [Bre74]. For the distribution of the number of events per unit drift time $\frac{d N}{d t}$ one can write

$$
\frac{d N}{d t}=\frac{d N}{d s} \cdot \frac{d s}{d t}=\frac{d N}{d s} \cdot \omega(t)
$$

with $s$ being the coordinate along the drift direction of the electrons and $\omega(t)$ is the drift velocity. Integration of this equation gives a relation of $\frac{d N}{d t}$ to the drift distance $d$

$$
d=\left(\frac{d N}{d s}\right)^{-1} \int_{t_{0}}^{t} \frac{d N}{d t^{\prime}} d t^{\prime}=\int_{t_{0}}^{t} \omega\left(t^{\prime}\right) d t^{\prime}
$$

where $t_{0}$ is the arrival time of the charged particle in the region of the corresponding wire and $t$ is the time when the pulse is generated at the wire. Therefore a constant $\frac{d N}{d s}$ distribution insures a proportionality between the drift distance $d$ and the integrated number of events per unit drift time $\frac{d N}{d t}$. Experimentally this uniformity is obtain by illuminating the entire VDC with a constant particle flux by using the inelastically scattered protons of the ${ }^{96}$ Mo target at an excitation energy of $\mathrm{E} \approx 30 \mathrm{MeV}$. At this energy the excitation spectrum is known to have an approximately constant distribution in dependence of the energy ('white spectrum' ) guaranteeing that the focal plane is illuminated with a constant flux. In Fig. 7.3 the measured drift time distribution is shown (left-hand side) and the corresponding lookup table (right-hand side) which has been created using Eq. 7.8. Clearly, one can identify two regions. In the first region the spectrum has a rather flat distribution. Here the electric field is constant and the field lines are parallel resulting in a constant drift velocity and hence a linear relationship between drift time and drift distance. In the second region - which is close to the signal wires - this linearity breaks down due to the inhomogeneity of the electric field.

In principle it is desirable to have one lookup table for each single wire. However, this requires a long measurement time to collect sufficient statistics in order to perform the procedure 

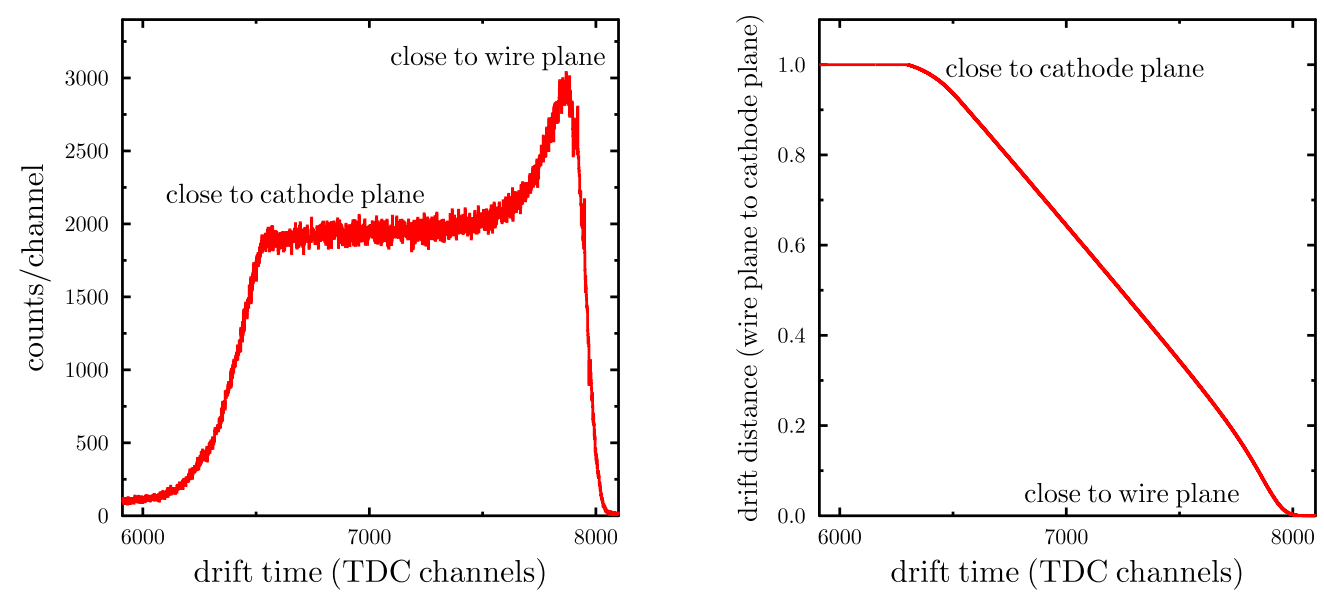

Figure 7.3: Drift-time spectrum (right-hand side) and associated lookup table of the $X_{2}$-wire plane (left-hand side).

outlined above. Furthermore the data analysis gets more complex. One way to overcome this problem, is to use one lookup table for each VDC. Since the drift times of each wire is converted into a drift distance using the same lookup table, it is required that all wires of one wire plane are time aligned. This time alignment has been done with a suitable algorithm which involves first of all a smoothing of the $\frac{d N}{d t}$ spectra of each wire and then searching the minimum gradient. Finally all spectra were aligned using this point of minimum gradient. For each wire plane one lookup table was created similarly to the one shown in Fig. 7.3.

\subsubsection{The lookup table shift}

It is necessary to measure absolute drift times in order to perform the analysis outlined in the last sections. It is physically impossible for the TDC module to measure a zero time difference. Hence a constant delay is introduced between the start and the stop signal either by the module itself or through different cable lengths. Consequently one has to correct the drift time spectrum shown on the left-hand side of Fig. 7.3 for this constant time offset. This is done by 'shifting' the lookup table either to right hand side - i.e. the TDC channel of the original time zero point was too small - or left hand side. There exist several methods to determine the correct value of this shift. In the present thesis the appropriate shift was determine by optimizing the resolution of the VDC defined as (see Fig. 7.2)

$$
\Delta=\frac{d_{i+1}-d_{i-1}}{2}-d_{i}
$$

For an correctly adjusted lookup table one expects the distribution of $\Delta$ to be a sharp peak centered around zero (see the red curve on the left-hand side of Fig. 7.4). If the time offset is not chosen correctly a double peak structure develops (blue curve). The origin of the double peak structure can be understood by examining the dependence of $\Delta$ on $b$-integer $(b)$ (see right-hand side of Fig. 7.4). The quantity $b$ denotes the calculated crossing point of the charged 

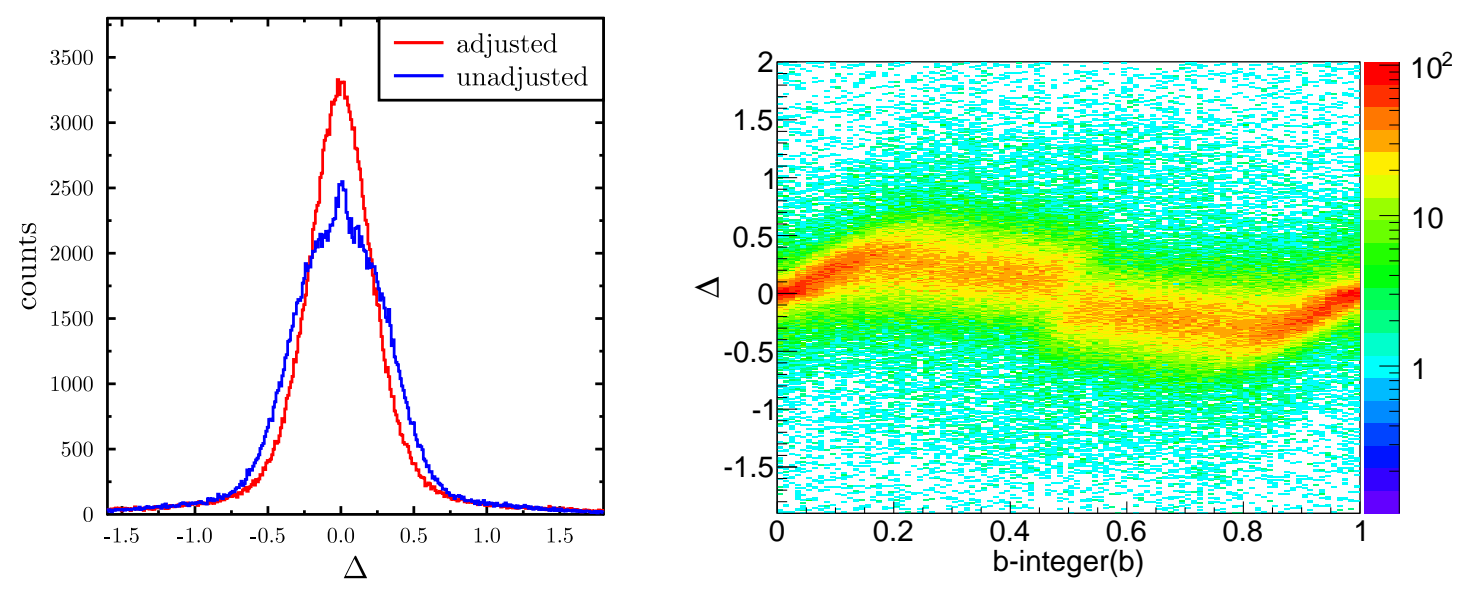

Figure 7.4: The lookup table in Fig. 7.3 is 'shifted' to correct for the constant time offset between the start and stop signals. This is done by inspecting the figures shown here. For more details see text.

particle with the VDC. Following the discussion in Ref. [Nev01] one can write for $\Delta^{\prime}$ based on an incorrectly shifted lookup table

$$
\begin{aligned}
\Delta^{\prime} & =\frac{\left(d_{i+1}+\zeta_{i+1}\right)-\left(d_{i-1}+\zeta_{i+1}\right)}{2}-\left(d_{i}-\zeta_{i}\right) \\
& =\left(\frac{\left(d_{i+1}-d_{i-1}\right.}{2}-d_{i}\right)+\left(\frac{\zeta_{i+1}-\zeta_{i-1}}{2}-\zeta_{i}\right) \\
& =\Delta+\left(\frac{\zeta_{i+1}-\zeta_{i-1}}{2}-\zeta_{i}\right),
\end{aligned}
$$

where $\zeta$ denotes the errors due to the wrong non-zero time offset. Clearly, the $\Delta$ distribution of the correct lookup table is changed because of the additional factor $\frac{\zeta_{i+1}-\zeta_{i-1}}{2}-\zeta_{i}$. It can be shown [Nev01] that this factor changes its sign when going from $b$-integer $(b)<0.5$ to $b$-integer $(b)>0.5$ and thus causes the double peak structure seen in Fig. 7.4.

Inspecting by eye similar figures like Fig. 7.4 the time offsets were adjusted for each wire chamber.

\subsection{Calibration of the scattering angle}

It is useful to know the scattering angle $\theta_{\mathrm{SCAT}}$ at the target position, in order to correct e.g. higher order aberrations of the spectrometer. This scattering angle has to be reconstructed using focal plane coordinates and angles measured using the three VDCs. Due to the complicated ion optics of the K600 and changing conditions from experiment to experiment it is not feasible to give a relationship between these quantities and $\theta_{\text {SCAT }}$ by means of a simulation, i.e. one has to conduct a calibration measurement for determining this dependence.

The brass plate ('the pepperpot') shown on the right-hand side of Fig. 7.5 is positioned downstream of the the target position at the entrance of the K600 spectrometer. The 29 holes at precisely known positions let only protons pass which meet specific horizontal scattering angles $\theta_{\text {th }}$ and vertical scattering angles $\theta_{\mathrm{tv}}$. The first hole from the middle in the horizontal (vertical) 

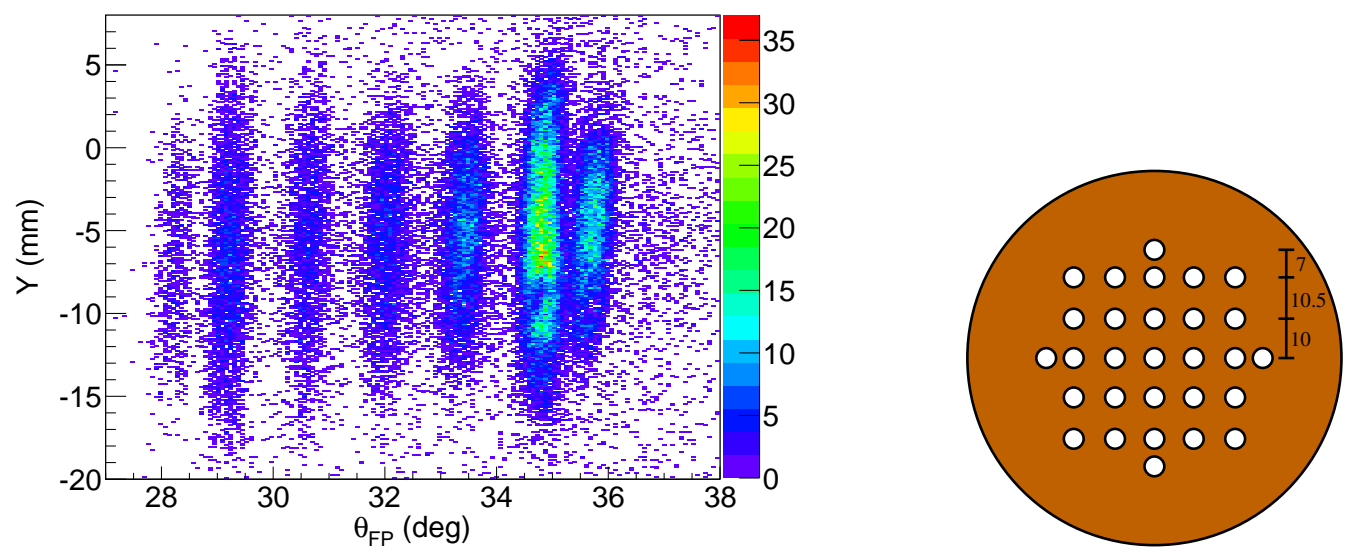

Figure 7.5: Image of the 'pepperpot' plate (right-hand side) on the focal plabe (left-hand side). The known positions of the holes of the 'pepperpot' plate are used to calibrate the scattering angle. The caption of the 'pepperpot' plate are in $\mathrm{mm}$.

direction are equivalent to a $\theta_{\mathrm{th}}\left(\theta_{\mathrm{tv}}\right)$ value of $\pm 0.819^{\circ}$, the second one is at $\pm 1.679^{\circ}$ and the third is at $\pm 2.251^{\circ}$ (relative to the center hole).

It is well known from previous experiments that the horizontal target scattering angle $\theta_{\text {th }}$ depends primarily on the horizontal focal plane angle $\theta_{\mathrm{FP}}$ and also slightly on the horizontal focal plane position $X$. This relationship can be described by the equation (which can be considered as a Taylor expansion of the unknown function)

$$
\theta_{\mathrm{th}}\left(\theta_{\mathrm{FP}}, X\right)=a+b \cdot X+c \cdot \theta_{\mathrm{FP}}+d \cdot X \cdot \theta_{\mathrm{FP}}
$$

The parameters $a, b, c$ and $d$ have to be determined through a calibration measurement using the 'pepperpot' plate. The left hand side of Fig. 7.5 displays its image on the focal plane. As expected $\theta_{\text {th }}$ depends strongly on $\theta_{\mathrm{FP}}$ and is almost independent of $Y$. The distribution was projected on the $\theta_{\mathrm{FP}}$-axis and the centroids of the 5 peaks assuming a Gaussian peak-shape (the outer holes were omitted) were determined. The focal plane position $X$ was kept constant in this analysis by gating on the elastically scattered protons only. In order to determine the $X$ dependence of Eq. 7.11 the measurement was repeated for different magnetic field, resulting in different $X$-positions over the whole acceptance region. In total 20 data points were collected to fix the parameters of Eq. 7.11.

It should be noted that during the analysis at no stage the vertical target scattering angle $\theta_{\mathrm{tv}}$ or the vertical focal plane angle has been determined. These angles become important for measurements under $\theta_{\mathrm{SCAT}}=0^{\circ}$ [Pol11] for background suppression. For experiments at $\theta_{\mathrm{T}}>8^{\circ}$ - like in the present experiment - it is of secondary importance.

\subsection{Correction of higher-order aberrations and kinematic effects}

Ideally one would like to have the situation where all protons - which left the target nuclei in the same excited state - are focused on the same $X$-position on the focal plane. This ideal case is altered by several effects which can not be corrected in the offline analysis like VDC resolution, beam stability, spectrometer resolution or straggling in the target. Also contributing is the dependence of the proton momentum on the scattering angle due to the recoil of the 

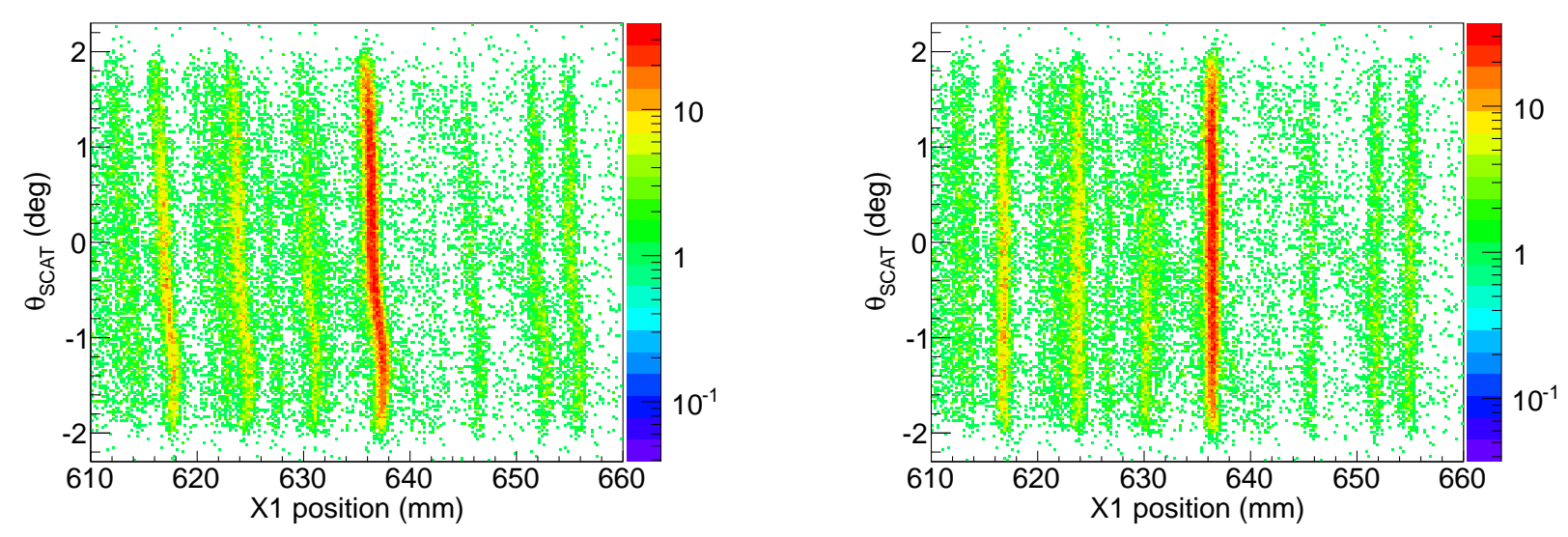

Figure 7.6: Uncorrected (left-hand side) and corrected (right-hand side) $\theta_{\mathrm{SCAT}}-\mathrm{X} 1$ spectra.

target nucleus and the aberrations of the spectrometer. Both can be corrected to obtain the optimal energy resolution.

On the left hand side of Fig. 7.6 is shown how strong the line shape of each nuclear state is curved in the $\theta_{\text {SCAT }}-X$-plane. This dependence can be corrected by fitting a high order polynomial on the curved line shape resulting in straight lines and improving significantly the energy resolution as shown on right hand side of Fig. 7.6. In the present thesis this polynomial was solely fitted to the strongly excited $3^{-}$state and assumed to be the same for all other nuclear states. This independence on the $X$-position is known to be incorrect [Pol11]. However, the chosen procedure is sufficient since the region of interest is in a very limited energy range from 1-3 MeV.

\subsection{Calibration of the focal plane}

In order to determine the excitation energies of the measured nuclear states one has to calibrate the focal plane in terms of the proton momentum $p$. It can be shown that the proton momentum is related to the horizontal focal plane position $X$ through a second order polynomial [Nev01]

$$
p(X)=a+b \cdot X+c \cdot X^{2}
$$

In case of ${ }^{94} \mathrm{Zr}$ and ${ }^{70} \mathrm{Zn}$ the calibration was achieved through ${ }^{24} \mathrm{Mg}\left(p, p^{\prime}\right)$. The positions of the well known states of ${ }^{24} \mathrm{Mg}$ were determined by means of a chi-square fit of a Gaussian function to the peak shape. Subsequently Eq. 7.12 was fitted to the $(p, X)$-tuples. Eq. 7.12 allows to assign a momentum to the protons scattered of ${ }^{94} \mathrm{Zr}$ and ${ }^{70} \mathrm{Zn}$ and the corresponding excitation energy can then be calculate using relativistic kinematics. The measurement of the excitation energies is estimated to have an error of $10-20 \mathrm{keV}$.

For the experiment of ${ }^{96} \mathrm{Mo}$ no ${ }^{24} \mathrm{Mg}$ calibration runs are available. This was due to the limited amount of time during the experiment. Therefore the calibration was done using known and easily identifiable states of ${ }^{96}$ Mo like the strongly excited $2_{1}^{+}$and $3_{1}^{-}$states. This works well for excited states with an energy smaller than $\sim 3 \mathrm{MeV}$. The errors are similar to that of ${ }^{94} \mathrm{Zr}$ and ${ }^{70} \mathrm{Zn}$. 


\subsection{Measured spectra}

In order to obtain the final spectra all runs corresponding to one scattering angle and target have to be summed. Some examples of spectra of the nuclei ${ }^{92,94} \mathrm{Zr},{ }^{94,96} \mathrm{Mo}$ and ${ }^{70} \mathrm{Zn}$ at various angles are presented in Figs. 7.7 and 7.8. The top part of Fig. 7.7 displays a ${ }^{96}$ Mo spectrum over the full acceptance up to $\sim 22 \mathrm{MeV}$. The spectrum can be decomposed in two parts. A low-lying part up to $\sim 5 \mathrm{MeV}$ where discrete states can be seen and a higher lying part where the high level density prevents the identification of individual nuclear states. The bottom part of Fig. 7.7 magnifies the energy region between 7 and $16 \mathrm{MeV}$. The broad structure is expected to be the isoscalar giant quadrupole resonance which has the maximum of its cross section at $10^{\circ}$. The giant resonance region was not of interest in this work and techniques like the wavelet analysis [She05] were not applied. The low-lying parts of all five investigated nuclei are shown

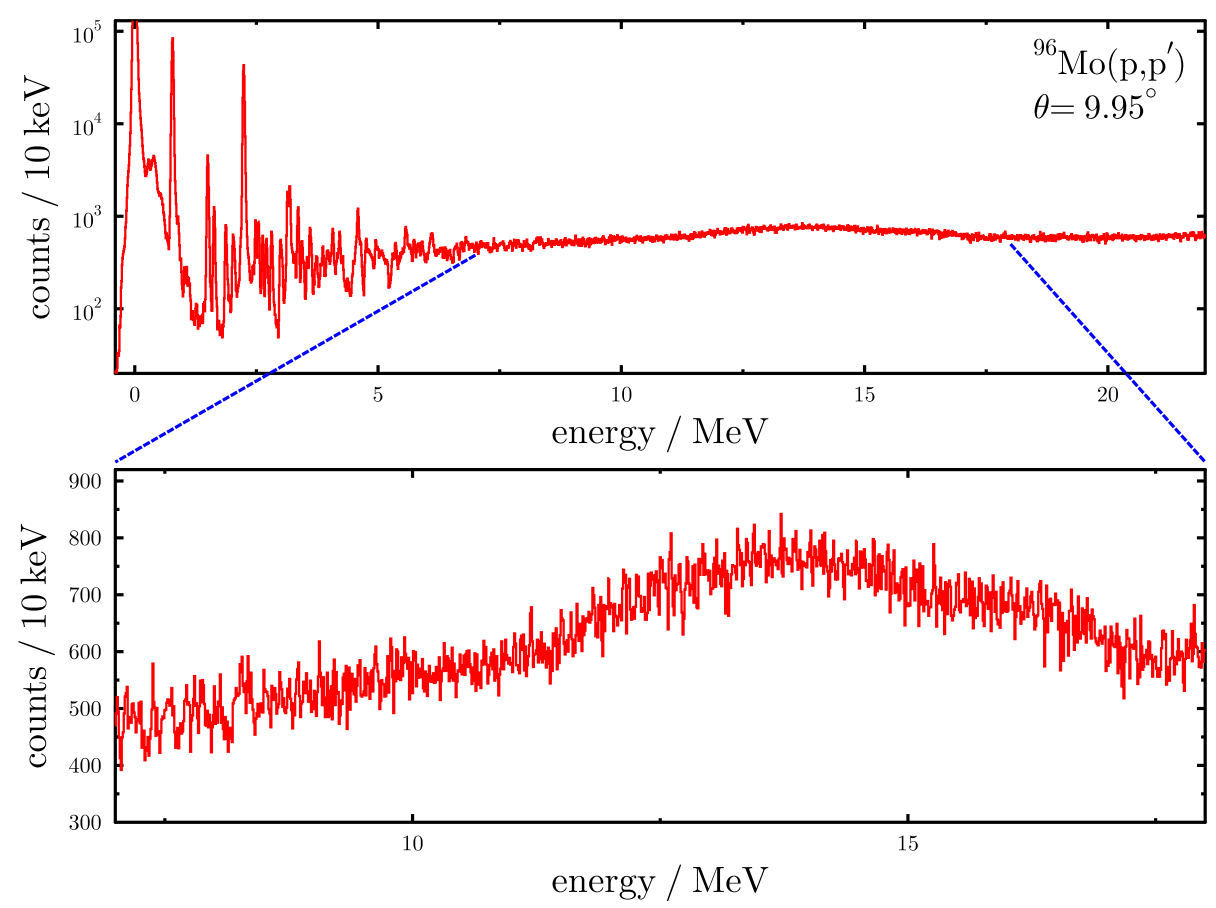

Figure 7.7: Excitation spectra of ${ }^{96} \mathrm{Mo}\left(p, p^{\prime}\right)$ over the full momentum acceptance (top). The region of the isoscalar giant quadrupole resonance if magnified (bottom).

in Fig. 7.8. Some important states are labeled with a number corresponding to a number in Tabs. 7.1- 7.5 for identification purposes. The main focus of this work is on the symmetric and mixed-symmetric one-phonon quadrupole states denoted with numbers 2 and 5 for ${ }^{92} \mathrm{Zr}, 2$ and 4 for ${ }^{94} \mathrm{Zr}$, 2 and 5 for ${ }^{94} \mathrm{Mo}$ and 2 and 5 for ${ }^{70} \mathrm{Zn}$. It was not possible to detect the mixed-symmetry state of ${ }^{96}$ Mo due to its very low cross section.

Very striking is the difference in energy resolution. While for ${ }^{92} \mathrm{Zr}$, ${ }^{94,96} \mathrm{Mo}$ and ${ }^{70} \mathrm{Zn}$ an energy resolution of $30-50 \mathrm{keV}$ was achieved, the data for ${ }^{94} \mathrm{Zr}$ range from 50 to $80 \mathrm{keV}$. This was because of problems with an unstable beam conditions, which could not be resolved during the beam time. Nevertheless it was possible to extract the cross sections of the $2_{1}^{+}$and $2_{\mathrm{ms}}^{+}$states reliably. 


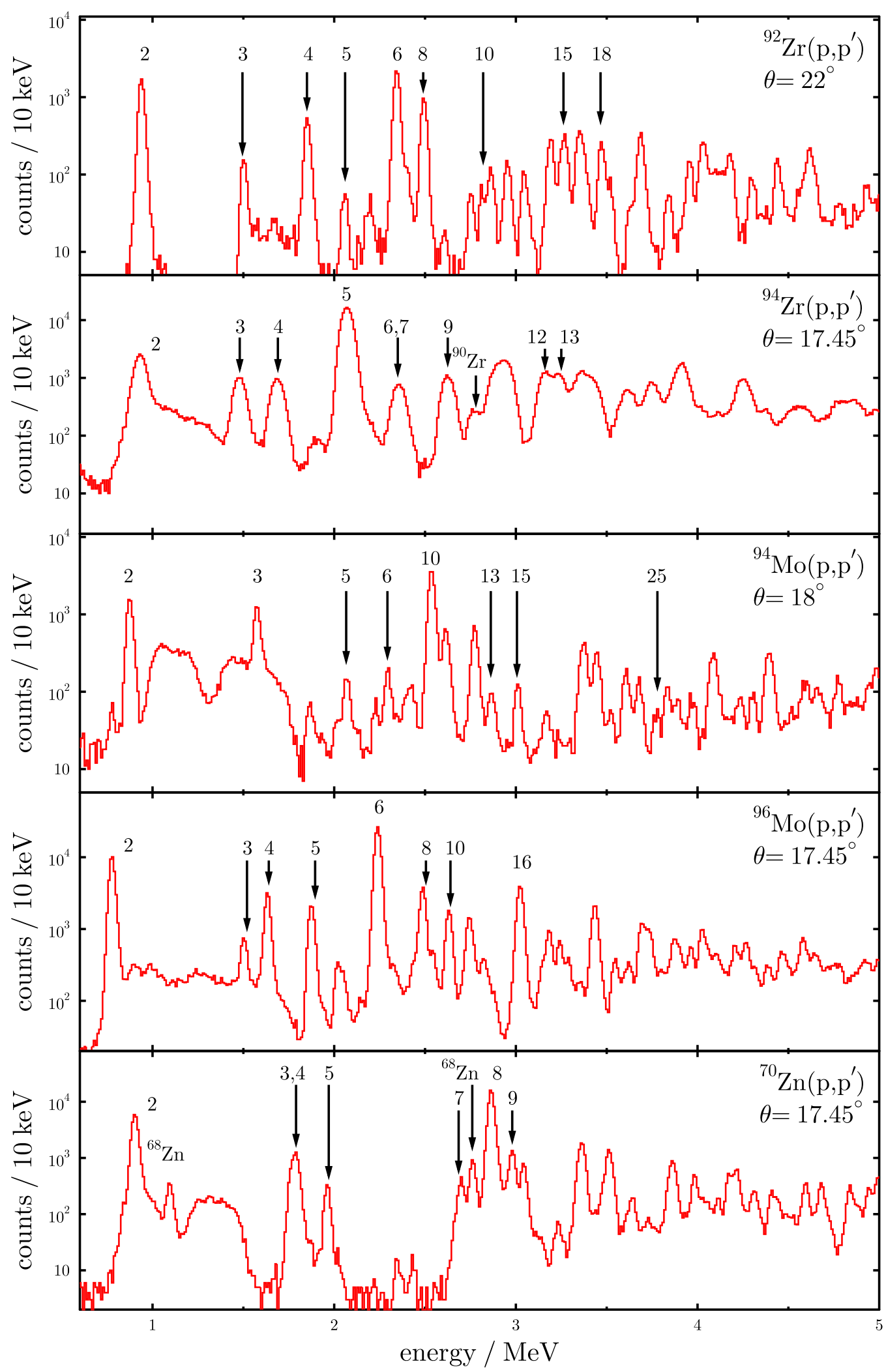

Figure 7.8: Examples energy spectra of ${ }^{92,94} \mathrm{Zr}$, ${ }^{94,96} \mathrm{Mo}$ and ${ }^{70} \mathrm{Zn}$ in the energy range $0.6-5 \mathrm{MeV}$. Some states are labeled with numbers referring to the numbers is Tabs. 7.1- 7.5. 


\subsection{Absolute cross sections}

The absolute differential cross section of each state in the center-of-mass system is written as (in $\mathrm{mb} / \mathrm{sr}$ ) [New96]

$$
\frac{d \sigma}{d \Omega}\left(\theta_{\text {c.m. }}\right)=\frac{10^{27} \cdot J \cdot N_{\text {counts }} \cdot \cos \left(\frac{\phi}{2}\right)}{N_{\mathrm{p}} \cdot \Delta \Omega \cdot n_{\mathrm{t}} \cdot T_{\mathrm{K} 600} \cdot \epsilon_{\mathrm{P}} \cdot \epsilon_{\mathrm{VDC}}} .
$$

The parameters entering in Eq. 7.13 and its associated uncertainties are discussed in the following.

- $J$ is the Jacobian - calculated with relativistic kinematics - to transform the cross section from the laboratory to the center- of-mass system. Uncertainties arise because of the imprecisely known scattering angle $\theta_{\mathrm{SCAT}}$ and the energy spread of the beam. However the error is estimated to be $<0.01 \%$ and is therefore negligible.

- $N_{\text {counts }}$ is the number of counts of the state of interest. They were obtain from the kind of spectra shown in Fig. 7.8 by fitting a Gaussian function to the peak shapes with the program Gaspan [Rie]. The spectra were divided in several regions and within them the FWHM of the Gaussian function was kept constant. The uncertainty of the yields were calculated from the uncertainties of the fit parameters.

- $N_{p}$ is the total number of protons incident on the target. This quantity is obtained from the collected charge at the beam dump from the current integrator unit. The current integrator gives a rate between $0-1000 \mathrm{~Hz}$ which is proportional to the current. The number of protons is then given by the equation

$$
N_{p}=\frac{\mathrm{CII} \cdot R}{1000 \cdot \mathrm{e}},
$$

where $e$ is the elementary charge, $R$ (in $\mathrm{nA}$ ) the is full scale setting of the current integrator and CII is the reading of the scaler which is connected to the output of the current integrator inhibited with the DAQ busy signal, i.e. at this point the dead time of the setup is taken into account. The dead time was typically smaller than $2 \%$. The uncertainty in the collected charge was taken to be $0.5 \%$ [New96].

- $\Delta \Omega$ (in msr) is solid angle covered by the spectrometer. It amounts to $3.48 \mathrm{msr}$ and the error was taken to be $0.3 \%$ [Nev11].

- $n_{\mathrm{t}}$ is the number of target nuclei per unit volume which can be calculated using the equation

$$
n_{t}=\frac{N_{A} \cdot t \cdot \epsilon_{d}}{M},
$$

with $N_{A}$ being the Avogadro constant, $t$ is the target thickness in $\mathrm{g} / \mathrm{cm}^{2}, d$ is the enrichment of the target and $M$ is the atomic mass. For $\mathrm{t}$, the value specified by the manufacturer was taken, with an uncertainty of $5 \%$.

- The transmission of the K600 spectrometer $T_{\mathrm{K} 600}$ as well as the paddle efficiency (of the two scintillator on the focal plane) was taken to be 100\% [New96]. 
- $\epsilon_{\mathrm{VDC}}$ is the total efficiency of all VDCs. The intrinsic efficiency of wire chamber $X 1$ is given by

$$
\epsilon_{\mathrm{X} 1}=\frac{N_{\mathrm{X} 1, \mathrm{X} 2}}{N_{\mathrm{X} 1, \mathrm{X} 2}+N_{\overline{\mathrm{X}} 1, \mathrm{X} 2}} .
$$

$N_{\mathrm{X} 1, \mathrm{X} 2}$ is the number of events where the $X 1$ - and $X 2$-chambers both produced a valid event, while $N_{\overline{\mathrm{X} 1}, \mathrm{X} 2}$ is the number of events where only $X 2$ has a valid event but not $X 1$. A similar equation holds for wire chamber $X 2$. It should be noted that at no point in the analysis the information of wire chamber $U 2$ was used (which is only necessary to calculate the $Y$-coordinate). A gate on a specific $Y$-range can help to improve the peak to background ratio but in the present experiment this was not the case. Hence the wire chamber $U 2$ was ignored improving the statistics.

The final total efficiency is given through

$$
\epsilon_{\mathrm{VDC}}=\epsilon_{\mathrm{geo}} \cdot \epsilon_{\mathrm{X} 1} \cdot \epsilon_{\mathrm{X} 2}
$$

The geometric efficiency $\epsilon_{\text {geo }}$ was taken to be $100 \%$ [New96]. The efficiency of the $X_{1}$ and $X_{2}$-chambers was usually $88-90 \%$ and therefore the total efficiency amounts to $\sim 80 \%$. The error is estimated to be $1 \%$.

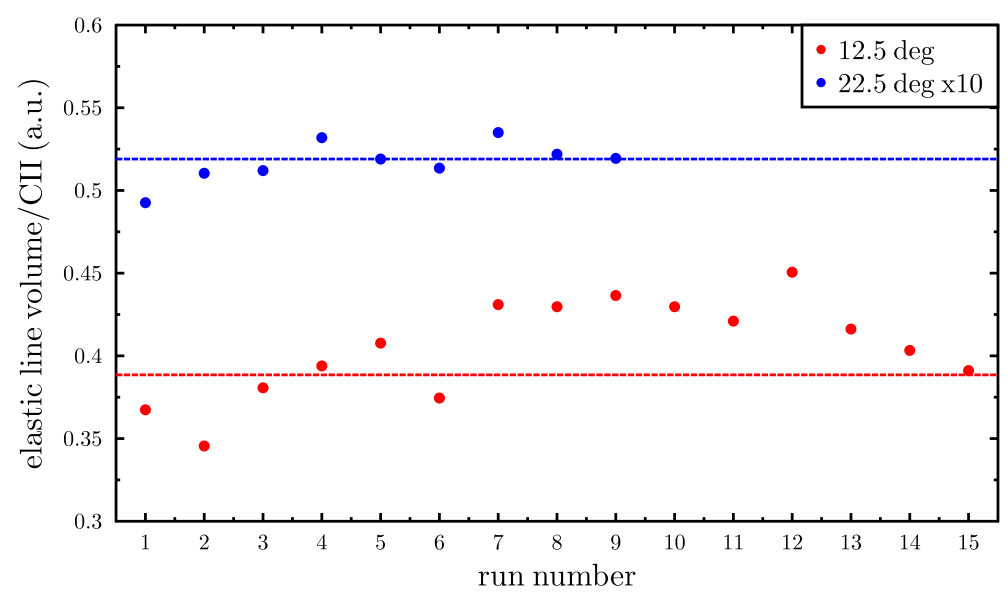

Figure 7.9: Flucation of the absolute cross section with the run number for two different scattering angles.

The present experiment had a general normalization problem. In principle one would expect the ratio $N_{\text {counts }} / N_{p}$ to be constant over time for a given state and a given angle. Surprisingly this ratio fluctuated over time during the experiment. Fig. 7.9 presents the dependence of the ratio of the yield of the elastic peak and the inhibited scaler signal (which is - according to Eq. 7.14 - proportional to $N_{p}$ ) on the run number for two different angles. For an angle of $22.5^{\circ}$ the deviations from the ratio of the full measurement is on average $1.7 \%$, i.e. quiet acceptable. In contrast to $\theta=12.5^{\circ}$ where the deviations are significantly higher with $7.3 \%$. Typically the deviations where less than $4 \%$. To somehow account for this effect the deviations were included in the final error of the cross section. 


\subsection{Experimental results}

In the following the final results of the five proton scattering experiments are presented. The measured cross sections are compared to a collective model calculation using the program CHUCK3 [Kun]. A phenomenological optical models was used. Its parameters were determine to give a good description of the $2_{1}^{+}$and $3_{1}^{-}$states of each nucleus. The purpose of the investigation is to determine the spin and parity of each state and to compare the results to the findings of other experiments. It was assumed that only natural parity states were exicted in proton scattering at $200 \mathrm{MeV}$, i.e. spin $J$ and parity $\pi$ of a given state are connected via $\pi=(-1)^{J}$. The cross sections of all states were a firm or tentative spin assignment was possible are given in the appendix B.

7.8.1 Results of the ${ }^{92} \mathrm{Zr}\left(p, p^{\prime}\right)$ experiment

\begin{tabular}{|c|c|c|c|c|c|c|}
\hline No & $\begin{array}{c}E_{x} \\
(\mathrm{keV})^{1}\end{array}$ & $\begin{array}{c}E_{x} \\
(\mathrm{keV})^{2}\end{array}$ & $\begin{array}{c}E_{x} \\
(\mathrm{keV})^{3}\end{array}$ & $\overline{J^{\pi 1}}$ & $\overline{J^{\pi 2}}$ & 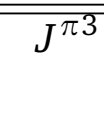 \\
\hline 1 & 0 & 0 & 0 & $0^{+}$ & $0^{+}$ & $0^{+}$ \\
\hline 2 & $934(10)$ & $935(10)$ & $934.46(10)$ & $2^{+}$ & $2^{+}$ & $2^{+}$ \\
\hline 3 & $1490(10)$ & $1495(10)$ & 1495.5(1) & $4^{+}$ & $4^{+}$ & $4^{+}$ \\
\hline 4 & $1848(10)$ & 1847(10) & 1847.3(1) & $2^{+}$ & $2^{+}$ & $2^{+}$ \\
\hline 5 & $2059(10)$ & $2053(10)$ & $2066.6(1)$ & $2^{+}$ & $2^{+}$ & $2^{+}$ \\
\hline 6 & $2340(10)$ & $2334(10)$ & $2339.6(1)$ & $3^{-}$ & $3^{-}$ & $3^{-}$ \\
\hline 7 & $2393(10)$ & $2393(10)$ & $2398.4(1)$ & $4^{+}$ & $4^{+}$ & $4^{+}$ \\
\hline 8 & $2488(10)$ & $2482(10)$ & $2485.9(2)$ & $5^{-}$ & $5^{-}$ & $5^{-}$ \\
\hline 9 & $2817(10)$ & $2823(10)$ & 2819.6(1) & $2^{+}$ & $2^{+}$ & $2^{+}$ \\
\hline 10 & $2862(10)$ & $2869(10)$ & $2864.7(2)$ & $4^{+}$ & $4^{+}$ & $4^{+}$ \\
\hline 11 & $2950(10)$ & 2963(10) & & $6^{+}$ & $\left(6^{+}\right)$ & \\
\hline 12 & $3050(10)$ & $3055(10)$ & $3057.5(3)$ & $2^{+}$ & $2^{+}$ & $2^{+}$ \\
\hline 13 & $3183(10)$ & $3187(10)$ & $3178.3(2)$ & $\left(4^{+}\right)$ & $4^{+}$ & $4^{+}$ \\
\hline 14 & $3238(20)$ & $3248(10)$ & & $\left(4^{+}, 5^{-}\right)$ & $4^{+}$ & \\
\hline 15 & $3262(20)$ & $3273(10)$ & $3262.9(4)$ & $2^{+}$ & $2^{+}$ & $2^{+}$ \\
\hline 16 & $3340(20)$ & $3345(10)$ & & $5^{-}$ & $5^{-}$ & \\
\hline 17 & $3372(20)$ & $3382(10)$ & & & $3^{-}$ & \\
\hline 18 & $3446(20)$ & $3452(10)$ & $3452.1(3)$ & $3^{-}$ & $3^{-}$ & $\left(2^{+}\right)$ \\
\hline 19 & $3488(20)$ & $3491(10)$ & $3500.1(3)$ & $2^{+}$ & $\left(3^{-}\right)$ & $2^{+}$ \\
\hline 20 & $3623(20)$ & $3634(10)$ & $3628.4(4)$ & & & $(2,3)$ \\
\hline 21 & $3644(20)$ & & $3640.3(4)$ & & & $\left(2^{+}\right)$ \\
\hline
\end{tabular}

Table 7.1: Energy, spin and parity assignments of the ${ }^{92} \mathrm{Zr}\left(p, p^{\prime}\right)$ experiment and comparison to other experiments. 
states at $934 \mathrm{keV}, 1490 \mathrm{keV}, 1848 \mathrm{keV}, 2059 \mathrm{keV}, 2340 \mathrm{keV}, 2393 \mathrm{keV}, 2488 \mathrm{keV}, 2817 \mathrm{keV}$ and $2862 \mathrm{keV}$.

All states are nicely described using the predicted cross section of the collective model. Each assigned spin and parity agree with the results of Refs. [Sin86, Fra05]. The $2_{3}^{+}$at $2059 \mathrm{keV}$ is an exception. The description of its cross section assuming a direct excitation from the ground state is not acceptable. It is possible that this state is a member of the symmetric two-phonon triplet. Ref. [Fra05] identifies this state unambiguously as a $2^{+}$state and gives upper limits of the $B(E 2)$-value to the ground state and to the $2_{1}^{+}$state of $<0.005$ W.u. and $<16$ W.u. respectively. Both are in agreement with the expectations of a two-phonon state.
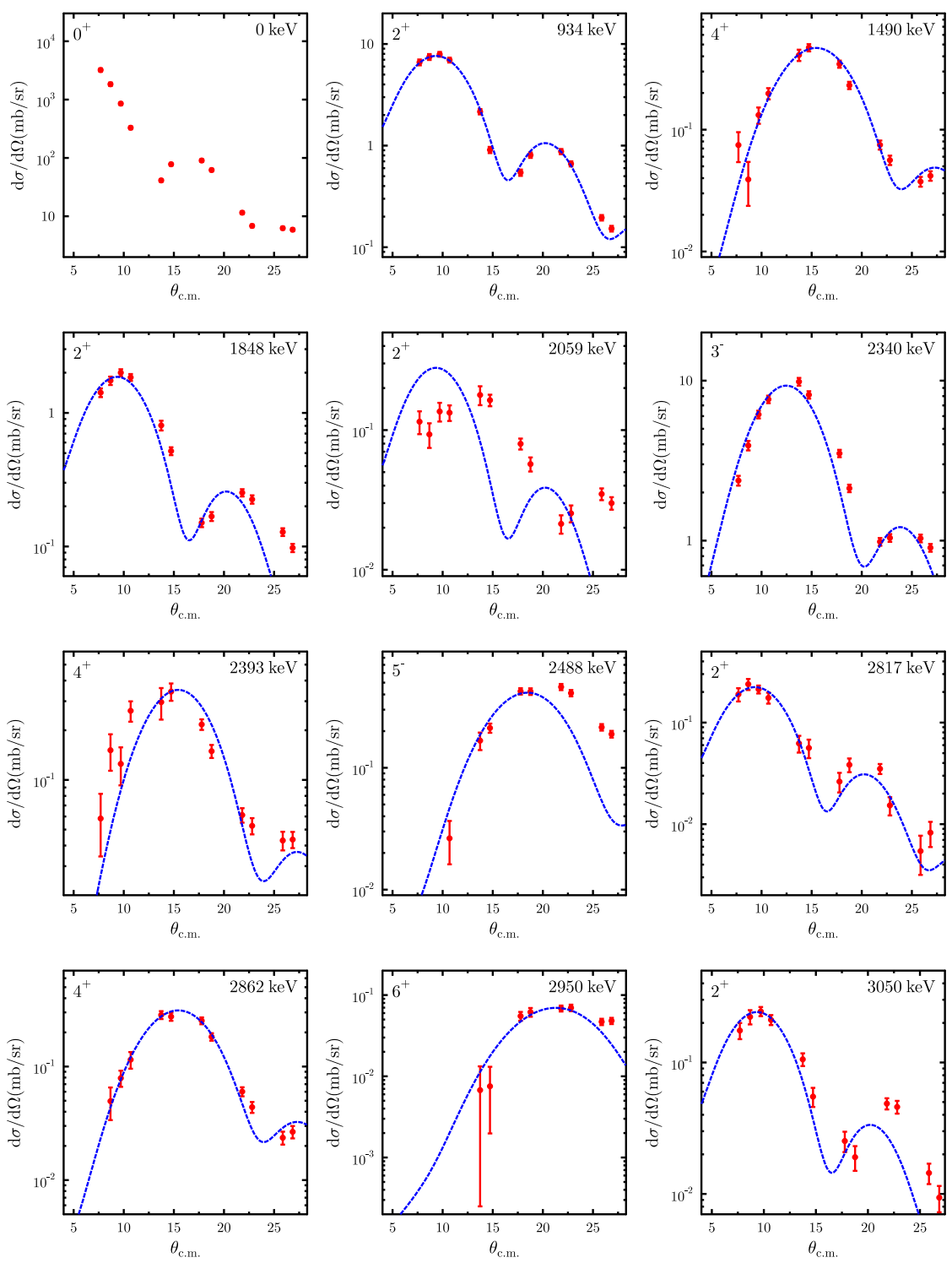

Figure 7.10: Angular distributions of ${ }^{92} \mathrm{Zr}\left(p, p^{\prime}\right)$. Shown are only states where a meaningful angular distribution could be measured. Calculations are done using a phenomenological optical potential and a collective model. 


\section{$6_{1}^{+}$state at $2950 \mathrm{keV}$.}

$\mathrm{J}^{\pi}=6^{+}$is assigned to this state. Ref. [Fra05] does not find evidence for this state while Ref. [Sin86] makes a tentative $\mathrm{J}^{\pi}=\left(6^{+}\right)$assignment.

\section{$2_{5}^{+}$state at $3050 \mathrm{keV}$.}

In agreement with Refs. [Fra05, Sin86] spin and parity $J^{\pi}=2^{+}$is assigned to this state.

tentative $4^{+}$state at $3183 \mathrm{keV}$.

There are clear deviations at larger scattering from the assumed $4^{+}$cross section. Hence only a tentative $J^{\pi}=\left(4^{+}\right)$assignment is made. Ref. [Fra05] as well as Ref. [Sin86] both find a $4^{+}$ state at this energy.

tentative $4^{+}, 5^{-}$state at $3238 \mathrm{keV}$.

Ref. [Sin86] reports a $4^{+}$state at $3248 \mathrm{keV}$. It was not possible to confirm this result unambiguously. The cross section is best described assuming quantum number $J^{\pi}=5^{-}$(green curve in Fig. 7.11). However also $J^{\pi}=4^{+}$(blue curve) cannot be ruled out.

$2_{6}^{+}$state at $3262 \mathrm{keV}$.

The angular distribution of the cross section is very well described assuming spin and parity $\mathrm{J}^{\pi}$ $=2^{+}$. This is the same result as the one of Refs. [Sin86, Fra05].

$5_{1}^{-}$state at $3340 \mathrm{keV}$.

Despite some deviations $J^{\pi}=5^{-}$is assigned to this state. This is in agreement with Ref. [Sin86]. state at $3372 \mathrm{keV}$.

It was not possible to extract a meaningful angular distribution for this state. Ref. [Sin86] finds evidence of a $3^{-}$state at a slightly higher energy of $3382 \mathrm{keV}$.

$3_{2}^{-}$state at $3446 \mathrm{keV}$.

This strongly excited $3^{-}$state is also seen by Ref. [Sin86]. However Ref. [Fra05] finds a tentative $J^{\pi}=\left(2^{+}\right)$state at this energy.
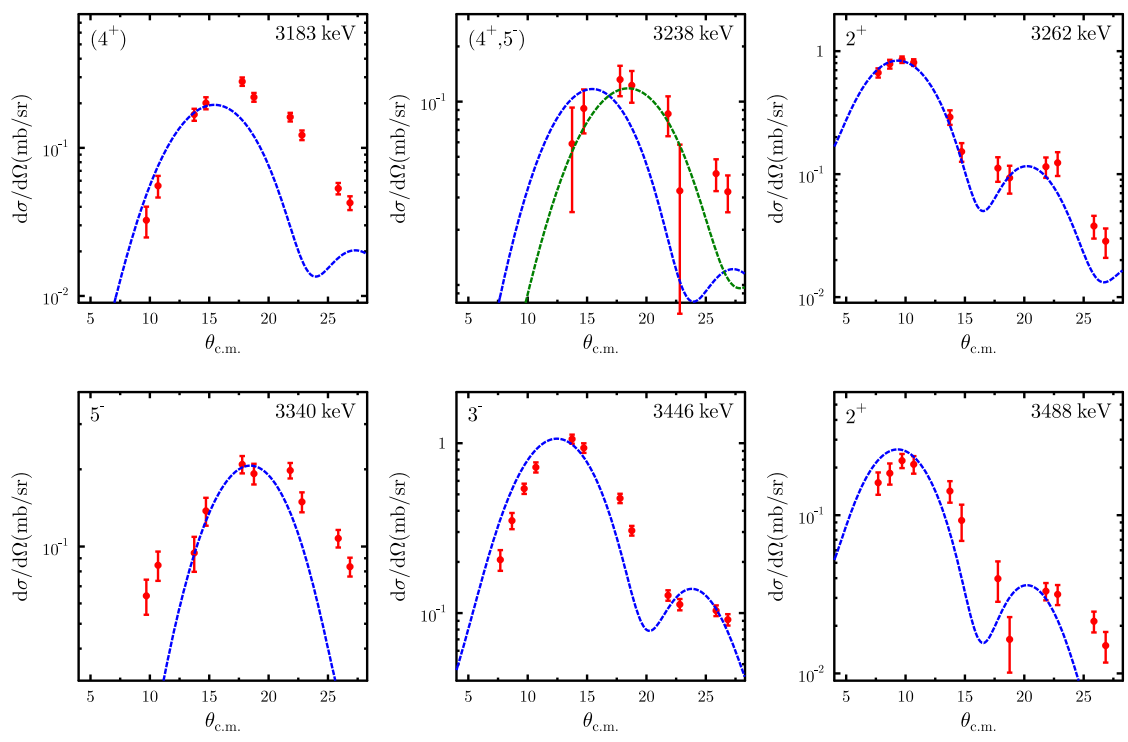

Figure 7.11: Same as Fig. 7.10.

$2_{7}^{+}$state at $3488 \mathrm{keV}$.

$J^{\pi}=2^{+}$of Ref. [Fra05] is confirmed. Ref. [Sin86] finds a tentative $J^{\pi}=\left(3^{-}\right)$state at the same energy. 
states at $3623 \mathrm{keV}$ and $3644 \mathrm{keV}$.

For both states no meaningful angular distribution is obtained. Ref. [Sin86] finds evidence of a tentative $J=(2,3)$ at $3628.4(4) \mathrm{keV}$ and a $J^{\pi}=\left(2^{+}\right)$state at $3640.3(4) \mathrm{keV}$.

\subsubsection{Results of the ${ }^{94} \mathrm{Zr}\left(p, p^{\prime}\right)$ experiment}

$2_{1}^{+}$at $909 \mathrm{keV}$.

The cross section is described well using a collective model and a phenomenological optical potential assuming a spin of 2 and positive parity. This is in agreement with the results of Refs. [Sin86, Elh08].

$4_{1}^{+}$at $1466 \mathrm{keV}$.

In agreement with Refs. [Sin86, Elh08] the angular distribution can be well described assuming $J^{\pi}=4^{+}$.

$2_{2}^{+}$at $1668 \mathrm{keV}$.

This state is assumed to be the quadrupole one-phonon mixed symmetry state of ${ }^{94} \mathrm{Zr}$. Positive parity and $J=2$ of Refs. [Sin86, Elh08] are confirmed.

$3_{1}^{-}$at $2059 \mathrm{keV}$.

This strongly excited state is confirmed to be the $3_{1}^{-}$state in agreement with Refs. [Sin86,Elh08].

\begin{tabular}{c|ccc|ccc}
\hline \hline No & $\begin{array}{c}E_{x} \\
(\mathrm{keV})^{1}\end{array}$ & $\begin{array}{c}E_{x} \\
(\mathrm{keV})^{2}\end{array}$ & $\begin{array}{c}E_{x} \\
(\mathrm{keV})^{3}\end{array}$ & $J^{\pi 1}$ & $J^{\pi 2}$ & $J^{\pi 3}$ \\
\hline 1 & 0 & 0 & 0 & $0^{+}$ & $0^{+}$ & $0^{+}$ \\
2 & $909(20)$ & $919(10)$ & $918.82(2)$ & $2^{+}$ & $2^{+}$ & $2^{+}$ \\
3 & $1466(10)$ & $1469(10)$ & $1469.70(2)$ & $4^{+}$ & $4^{+}$ & $4^{+}$ \\
4 & $1668(10)$ & $1671(10)$ & $1671.45(2)$ & $2^{+}$ & $2^{+}$ & $2^{+}$ \\
5 & $2059(10)$ & $2057(10)$ & $2057.87(2)$ & $3^{-}$ & $3^{-}$ & $3^{-}$ \\
6 & & & $2151.34(2)$ & & & $2^{+}$ \\
7 & & $2336(10)$ & $2329.97(2)$ & $4^{+}$ & $4^{+}$ & $4^{+}$ \\
8 & & $2372(10)$ & $2366.34(2)$ & $\left(2^{+}\right)$ & $2^{+}$ & $2^{+}$ \\
9 & $2615(10)$ & $2617(10)$ & $2605.39(3)$ & $5^{-}$ & $5^{-}$ & $5^{-}$ \\
10 & $\sim 2861$ & $2881(10)$ & $2873.65(3)$ & & $4^{+}$ & $\left(4^{+}\right)$ \\
& & & $2888.25(7)$ & & & $4^{+}$ \\
11 & $\sim 2923$ & $2940(10)$ & $2908.04(2)$ & & $5^{-}$ & $2^{+}$ \\
& & & $2927.50(5)$ & & & $3^{-}$ \\
12 & $3154(10)$ & $3163(10)$ & $3155.93(3)$ & $4^{+}$ & $4^{+}$ & $\left(4^{+}\right)$ \\
13 & $3232(10)$ & $3244(10)$ & $3224.84(4)$ & $3^{-}$ & $3^{-}$ & $\left(4^{+}\right)$ \\
\hline 1 \\
2 this work \\
Ref. [Sin86]
\end{tabular}

Table 7.2: Energy, spin and parity assignments of the ${ }^{94} \mathrm{Zr}\left(p, p^{\prime}\right)$ experiment and comparison to other experiments.

$2_{3}^{-}$at $2151 \mathrm{keV}$.

According to Tab. 8.3 this well-known $2^{+}$state has a small decay branch to the ground state 
of $B(E 2)=0.019$ W.u. and a large transition strength to the $2_{1}^{+}$state indicating significant twophonon components in its wave function. Unfortunately - due to its proximity to the $3^{-}$state no meaningful angular distribution could be measured.

$4^{+}$and $2^{+}$states at $2330 \mathrm{keV}$ and $2366 \mathrm{keV}$.

These two states form a doublet which cannot be resolved. Since the energies are precisely known (see Ref. [Elh08]) it was possible to fit the doublet - fixing the peak positions - and measure the angular distributions. The angular distributions confirm the $J^{\pi}=4^{+}$assignment of Refs. [Sin86, Elh08] of the state at $2330 \mathrm{keV}$. For the state at $2366 \mathrm{keV}$ a tentative $J^{\pi}=2^{+}$ assignment is possible.
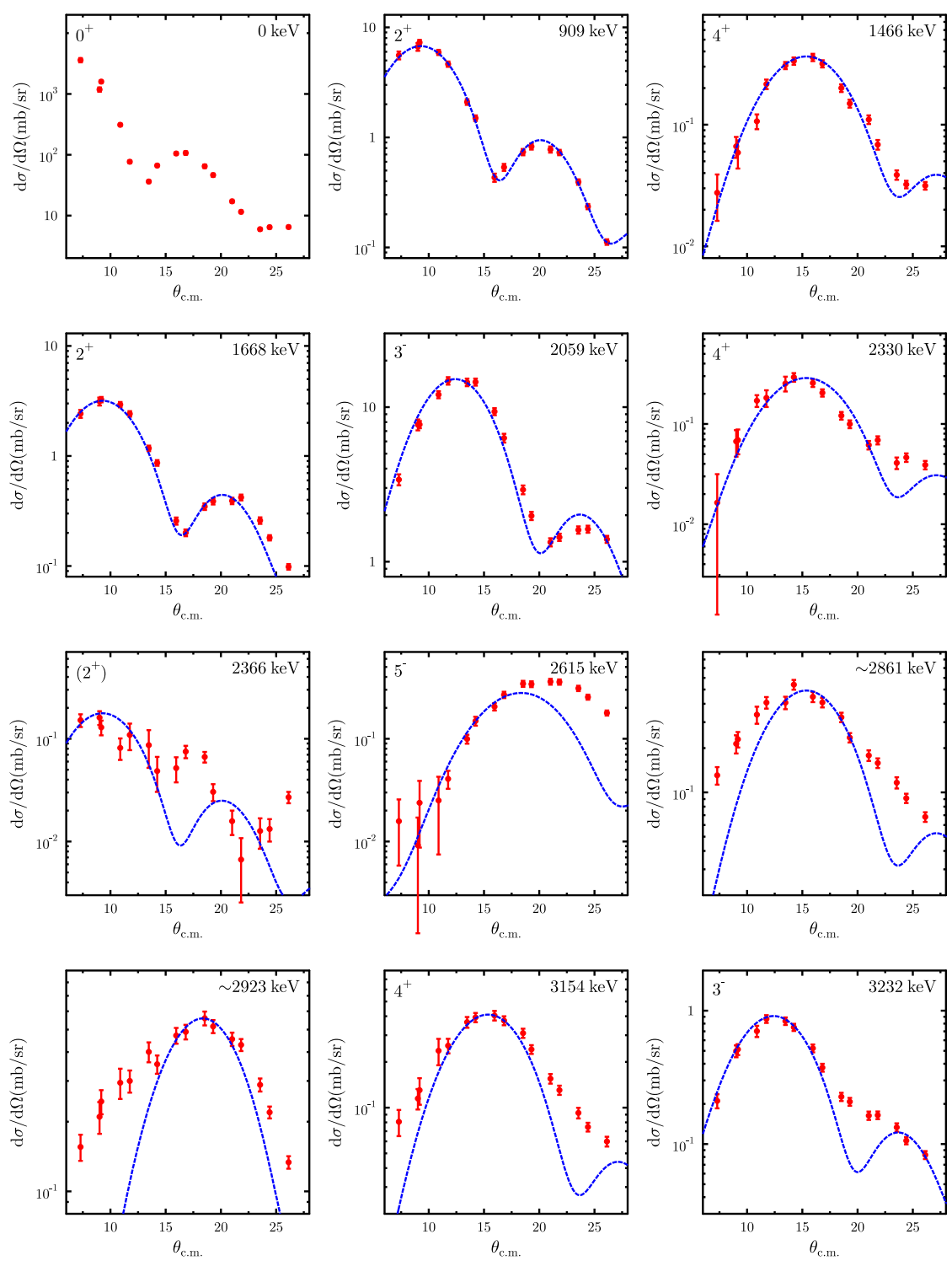

Figure 7.12: Same as Fig. 7.10 for ${ }^{94} \mathrm{Zr}\left(p, p^{\prime}\right)$.

\section{$5_{1}^{-}$at $2615 \mathrm{keV}$.}

The measured spin, parity and energy agree with a $5^{-}$state seen in Refs. [Sin86, Elh08].

States at $\sim 2861 \mathrm{keV}$ and $\sim 2923 \mathrm{keV}$.

In this region there are - according to Ref. [Elh08] - five close lying states which cannot be 
resolved. Indeed the measured angular distributions do not allow any spin assignments. It is possible, however, to draw conclusions about which states are excited strongest. In Fig. 7.12 it can be seen that the states around $\sim 2861 \mathrm{keV}$ are dominated by a $4^{+}$state and the states around $\sim 2923 \mathrm{keV}$ are dominated by a $5^{-}$state.

$4_{3}^{+}$at $3154 \mathrm{keV}$.

Despite some deviations at larger scattering angles positive parity and a spin of $J=4$ is assigned to this state. This is in agreement with the findings of Refs. [Sin86, Elh08], although Ref. [Elh08] makes only a tentative $J^{\pi}=4^{+}$assignment.

$3_{2}^{-}$at $3232 \mathrm{keV}$.

In agreement with Ref. [Sin86] $J^{\pi}=3^{-}$is assigned to this state. Ref. [Elh08] finds a tentative $4^{+}$state close in energy.

7.8.3 Results of the ${ }^{94} \mathrm{Mo}\left(p, p^{\prime}\right)$ experiment

$2_{1}^{+}$state at $872 \mathrm{keV}$.

In agreement with Refs. [Pig92, Fra03] a spin and parity of $J^{\pi}=2^{+}$could be assigned to this state.

$4_{1}^{+}$state at $1573 \mathrm{keV}$.

The angular distribution of the cross section is nicely described by the theoretical calculation assuming spin and parity $J^{\pi}=4^{+}$. This is same result as presented in Refs. [Pig92, Fra03].

tentative $2_{2}^{+}$state at $1865 \mathrm{keV}$.

According to Ref. [Fra03] this state is assumed to be a member of the symmetric two-phonon triplet. This is indicated by a large $B(E 2)$-value to the $2_{1}^{+}$state of $60_{-30}^{+20}$ W.u.. Indeed the angular distribution of the cross section clearly differs from the expectation of a one-phonon $2^{+}$state at larger scattering angles giving a hint for two-step contributions to the excitation mechanism of the state. In the present data there are indications that the $2^{+}$state at $1865 \mathrm{keV}$ is a doublet with an other close lying state at $\sim 1900$. A possible candidate is the $3_{1}^{-}$state of ${ }^{100} \mathrm{Mo}$ at an energy of $1908.28 \mathrm{keV}$. However it is difficult to make this observation quantitative because of the limited statistics. Nevertheless one should treat the measured cross section with care.

$2_{3}^{+}$state at $2066 \mathrm{keV}$.

This state is supposed to be the one-phonon $2^{+}$mixed-symmetry state as indicated by a large $B(M 1)$-value of $0.56(5) \mu_{N}^{2}$ to the $2_{1}^{+}$state [Fra03]. The measured cross section is clearly shifted to larger scattering angles with respect to the theoretical calculation although the shape is the same. This shift can be explained by a smaller matter transition radius than assumed in the collective model which is due to the mixed-symmetric character of this state (see Sect. 8.3). Hence spin and parity $J^{\pi}=2^{+}$is assigned.

$4_{2}^{+}$state at $2293 \mathrm{keV}$.

Only 7 data points could be measured for this state. These data points are nicely described by the collective model assuming spin and parity $J^{\pi}=4^{+}$. This is in agreement with Refs. [Pig92, Fra03].

state at $2347 \mathrm{keV}$.

No meaningful angular distribution could be extracted for this state. Ref. [Pig92] reports a state at a similar energy but without additional quantum numbers.

$2_{4}^{+}$state at $2391 \mathrm{keV}$.

$J^{\pi}=2^{+}$of Refs. [Pig92, Fra03] are confirmed. 


\begin{tabular}{|c|c|c|c|c|c|c|}
\hline No & $\begin{array}{c}E_{x} \\
(\mathrm{keV})^{1}\end{array}$ & $\begin{array}{c}\mathrm{E}_{x} \\
(\mathrm{keV})^{2}\end{array}$ & $\begin{array}{c}E_{x} \\
(k e V)^{3}\end{array}$ & $\overline{\mathrm{J}^{\pi 1}}$ & $\overline{\mathrm{J}^{\pi 2}}$ & $\overline{\mathrm{J}^{\pi 3}}$ \\
\hline 1 & 0 & 0 & 0 & $0^{+}$ & $0^{+}$ & $0^{+}$ \\
\hline 2 & $872(10)$ & $871(2)$ & $871.72(14)$ & $2^{+}$ & $2^{+}$ & $2^{+}$ \\
\hline 3 & $1573(10)$ & $1573(2)$ & $1573.72(14)$ & $4^{+}$ & $4^{+}$ & $4^{+}$ \\
\hline 4 & $1865(10)$ & $1864(2)$ & $1864.3(1)$ & $\left(2^{+}\right)$ & $2^{+}$ & $2^{+}$ \\
\hline 5 & $2066(10)$ & $2068(2)$ & 2067.4(1) & $2^{+}$ & $2^{+}$ & $2^{+}$ \\
\hline 6 & 2293(10) & $2295(2)$ & $2294.7(2)$ & $4^{+}$ & $4^{+}$ & $4^{+}$ \\
\hline 7 & $2347(20)$ & $2322(2)$ & & & & \\
\hline 8 & 2391(10) & $2393(2)$ & 2393.1(1) & $2^{+}$ & $2^{+}$ & $2^{+}$ \\
\hline 9 & 2423 & $2424(2)$ & $2423.4(2)$ & $6^{+}$ & $\left(5^{-}, 6^{+}\right)$ & $6^{+}$ \\
\hline 10 & 2534(10) & $2534(5)$ & 2533.8(3) & $3^{-}$ & $3^{-}$ & $3^{-}$ \\
\hline 11 & $2606(10)$ & 2611(5) & $2610.5(2)$ & $\left(5^{-}\right)$ & $5^{-}$ & $5^{-}$ \\
\hline 12 & $2763(10)$ & $2770(5)$ & $2768.2(2)$ & $4^{+}$ & $4^{+}$ & $4^{+}$ \\
\hline 13 & $2862(10)$ & $2854(5)$ & $\begin{array}{c}2870.02(2) \\
2872.4(2)\end{array}$ & $2^{+}+6^{+}$ & $\left(4^{+}\right)$ & $\begin{array}{l}2^{+} \\
6^{+}\end{array}$ \\
\hline 14 & $2946(10)$ & $2960(5)$ & & & $\left(4^{+}\right)$ & \\
\hline 15 & & 2996(5) & 2993.1(3) & & $\left(3^{-}\right)$ & $2^{+}$ \\
\hline & 2998(10) & $3014(5)$ & $3011.5(2)$ & $3^{-}$ & $3^{-}$ & $3^{-}$ \\
\hline 16 & $3086(20)$ & & & & & \\
\hline 17 & $3158(20)$ & & & & & \\
\hline 18 & $3190(20)$ & & & & & \\
\hline 19 & $3243(20)$ & & & & & \\
\hline 20 & $3365(20)$ & & & & & \\
\hline 21 & $3439(20)$ & & & $\left(4^{+}\right)$ & & \\
\hline 22 & $3518(20)$ & & & & & \\
\hline 23 & $3617(20)$ & & & & & \\
\hline 24 & $3688(20)$ & & & & & \\
\hline 25 & $3779(20)$ & & & $2^{+}$ & & \\
\hline 26 & $3842(20)$ & & & & & \\
\hline 27 & $3888(20)$ & & & & & \\
\hline 28 & $3974(20)$ & & & & & \\
\hline
\end{tabular}

Table 7.3: Energy, spin and parity assignments of the ${ }^{94} \mathrm{Mo}\left(p, p^{\prime}\right)$ experiment and comparison to other experiments.

$6_{1}^{+}$state at $2423 \mathrm{keV}$.

The angular distribution of the cross section give clear evidence of spin and parity $\mathrm{J}^{\pi}=6^{+}$. This is in agreement with Ref. [Fra03], while Ref. [Pig92] makes a tentative $J^{\pi}=\left(5^{-}, 6^{+}\right)$ assignment. The peak position was fixed to the known energy from gamma spectroscopy. Hence no independent energy was measured.

$3_{1}^{-}$state at $2534 \mathrm{keV}$.

This state is clearly the one-phonon $3_{1}^{-}$state of ${ }^{94} \mathrm{Mo}$. 

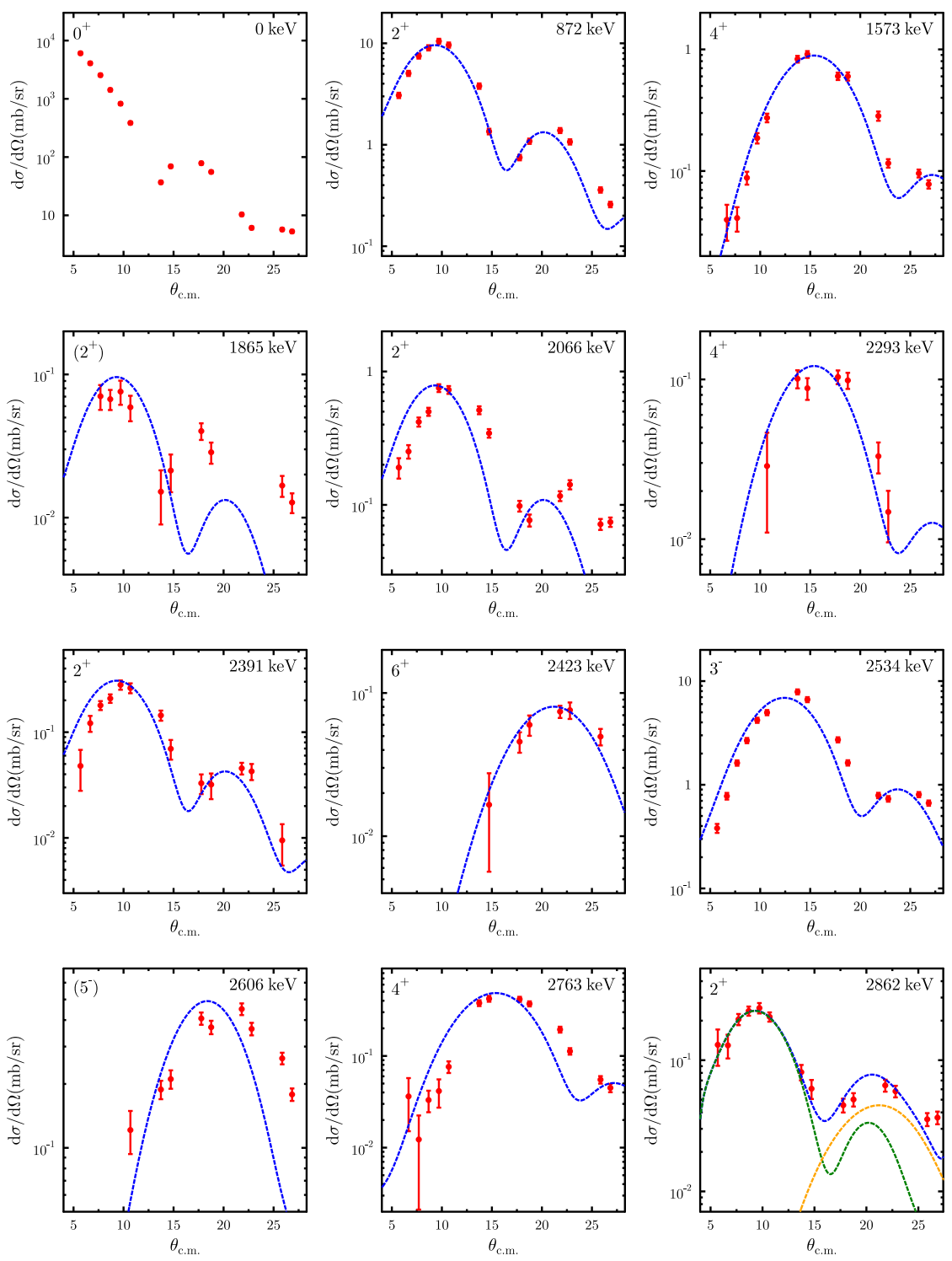

Figure 7.13: Same as Fig. 7.10 for ${ }^{94} \mathrm{Mo}\left(p, p^{\prime}\right)$.

tentative $5_{1}^{-}$state at $2606 \mathrm{keV}$.

The position of the maximum points to a $5^{-}$state. Due to deviations between theory and experiment this assignment is tentative. Refs. [Pig92, Fra03] find evidence of a $5^{-}$state at the same energy.

$4_{3}^{+}$state at $2763 \mathrm{keV}$.

$J^{\pi}=4^{+}$of Refs. [Pig92, Fra03] are confirmed.

doublet at $2862 \mathrm{keV}$.

According to Ref. [Fra03] this state is a doublet consisting of a $2^{+}$state and a $6^{+}$state at energies of $2870.02 \mathrm{keV}$ and $2872.4 \mathrm{keV}$ respectively. Due to the energy resolution of $29-42 \mathrm{keV}$ it was not possible to resolve the doublet in the present experiment. However the angular distribution is nicely described using a superposition (blue curve in Fig. 7.14) of a $2^{+}$(green curve) state and a $6^{+}$state (yellow curve). The $2^{+}$cross section dominates at smaller scattering angles while the $6^{+}$cross section dominates at larger scattering angles. 
state at $2946 \mathrm{keV}$.

No angular distribution could be obtained for this state. Ref. [Pig92] finds evidence of a tentative $4^{+}$state at a slightly higher energy.

$3_{2}^{-}$state at $2998 \mathrm{keV}$.

The gamma spectroscopic results of Ref. [Fra03] show a $2^{+}$state at 2993.1(3) keV and a $3^{-}$ state at 3011.5(2) keV. This is again a doublet which cannot be resolved. However the cross section is very well described by a $3^{-}$cross section only. This points to a much stronger excited $3^{-}$state.

states at $3086 \mathrm{keV}, 3158$ keV, 3190 keV, 3243 keV, 3365 keV, 3439 keV, 3518 keV, 3617 keV, 3688 $\mathrm{keV}, 3779 \mathrm{keV}, 3842 \mathrm{keV}, 3888 \mathrm{keV}$ and $3974 \mathrm{keV}$.

Only for two states above $3 \mathrm{MeV}$ spin and parity assignments could be made. The angular distribution of the cross sections give evidence of a tentative $4^{+}$state at $3439 \mathrm{keV}$ and a firm $2^{+}$ state at $3779 \mathrm{keV}$. Due to the small number of identified spins the comparison to the results of Refs. [Pig92, Fra03] is not done for those states.
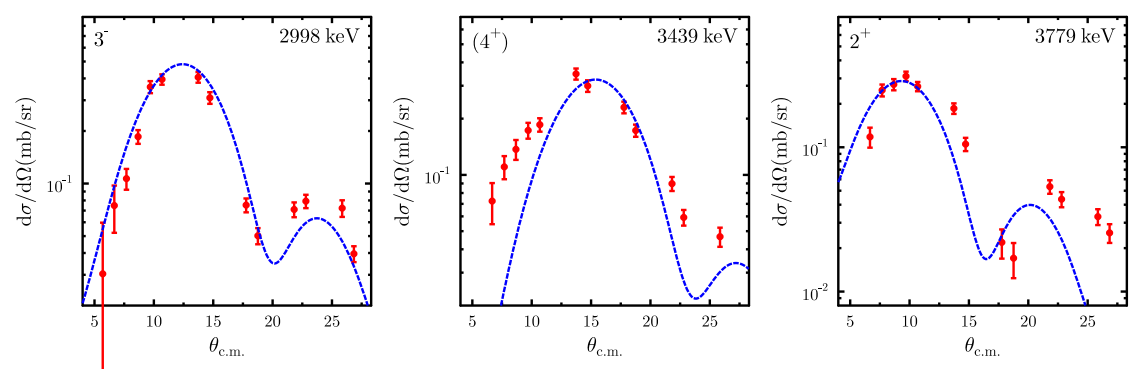

Figure 7.14: Same as Fig. 7.13.

\subsubsection{Results of the ${ }^{96} \mathrm{Mo}\left(p, p^{\prime}\right)$ experiment}

$2_{1}^{+}$at $773 \mathrm{keV}$.

In agreement with Refs. [Fre87, Les07] a spin and parity of $J^{\pi}=2^{+}$could be assigned.

$2_{2}^{+}$at $1498 \mathrm{keV}$.

The cross section is best described assuming $J^{\pi}=2^{+}$, although the features of the measured cross section are shifted to higher scattering angles. The reason might be a smaller transition radius as assumed by the collective model. Spin and parity assignments are in agreement with Refs. [Fre87, Les07].

$4_{1}^{+}$at $1628 \mathrm{keV}$.

At this energy a $2^{+}$state at $1625.88 \mathrm{keV}$ and a $4^{+}$state at $1628.20 \mathrm{keV}$ form a doublet (Ref. [Les07]) which cannot be resolved. However the cross section is well described assuming a $J=4^{+}$state only. This points to a weakly excited $2^{+}$state. This $2^{+}$state might have large two-phonon components because it is approximately at twice the energy of the $2_{1}^{+}$state.

$4_{2}^{+}$state at $1870 \mathrm{keV}$.

$J^{\pi}=4^{+}$of Refs. [Fre87, Les07] was confirmed by the good description of the measured cross section.

$3_{1}^{-}$state at $2235 \mathrm{keV}$.

The angular distribution of the cross section clearly indicates a $3^{-}$state. This is in agreement with Refs. [Fre87, Les07]. However Ref. [Les07] finds evidence for a $2^{+}$state at $2095.73 \mathrm{keV}$ 


\begin{tabular}{|c|c|c|c|c|c|c|}
\hline No & $\begin{array}{c}E_{x} \\
(\mathrm{keV})^{1}\end{array}$ & $\begin{array}{c}E_{x} \\
(\mathrm{keV})^{2}\end{array}$ & $\begin{array}{c}E_{x} \\
(\mathrm{keV})^{3}\end{array}$ & $J^{\pi 1}$ & $\overline{J^{\pi 2}}$ & $\overline{J^{\pi 3}}$ \\
\hline 1 & 0 & 0 & 0 & $0^{+}$ & $0^{+}$ & $0^{+}$ \\
\hline 2 & $773(20)$ & $778(5)$ & $778.22(5)$ & $2^{+}$ & $2^{+}$ & $2^{+}$ \\
\hline 3 & $1498(10)$ & 1498(5) & 1497.76(5) & $2^{+}$ & $2^{+}$ & $2^{+}$ \\
\hline \multirow[t]{2}{*}{4} & $1628(10)$ & $1627(5)$ & $1625.88(5)$ & $4^{+}$ & $4^{+}$ & $2^{+}$ \\
\hline & & & $1628.20(5)$ & & & $4^{+}$ \\
\hline 5 & $1870(10)$ & $1870(5)$ & 1869.53(5) & $4^{+}$ & $4^{+}$ & $4^{+}$ \\
\hline 6 & $2235(10)$ & $2234(5)$ & $2234.63(6)$ & $3^{-}$ & $3^{-}$ & $3^{-}$ \\
\hline 7 & $2428(10)$ & $2432(5)$ & $2426.07(6)$ & & $2^{+}$ & $2^{+}$ \\
\hline 8 & $2481(10)$ & $2481(5)$ & $2481.04(8)$ & $4^{+}$ & $4^{+}$ & $4^{+}$ \\
\hline 9 & $2539(10)$ & $2542(5)$ & $2540.34(5)$ & $\left(2^{+}\right)$ & & $\left(2^{+}, 3^{+}\right)$ \\
\hline 10 & $2626(10)$ & $2625(5)$ & $2625.23(8)$ & $4^{+}$ & $4^{+}$ & $4^{+}$ \\
\hline 11 & $2700(10)$ & & $2700.08(8)$ & $\left(2^{+}\right)$ & & $2^{+}$ \\
\hline \multirow[t]{2}{*}{12} & $2739(10)$ & $2734(5)$ & $2734.61(12)$ & $6^{+}$ & $\left(5^{-}\right)$ & $4^{+}$ \\
\hline & & & $2755.12(30)$ & & & $6^{+}$ \\
\hline 13 & $2809(10)$ & $2807(5)$ & $2818.53(10)$ & & $\left(3^{-}\right)$ & $4^{+}$ \\
\hline 14 & $2860(10)$ & $2875(5)$ & & & $\left(4^{+}, 6^{+}\right)$ & \\
\hline 15 & $2989(10)$ & 2981(5) & $2986.79(6)$ & & $1^{-}, 2^{+}$ & $2^{+}$ \\
\hline 16 & $3018(20)$ & $3020(5)$ & $3024.47(6)$ & $5^{-}$ & $4^{+}$ & $2^{+}$ \\
\hline 17 & $3137(20)$ & $3140(5)$ & $3134.50(7)$ & $2^{+}$ & & $2^{+}$ \\
\hline 18 & $3179(20)$ & $3182(5)$ & 3178.74(7) & $3^{-}$ & $3^{-}$ & $3^{-}$ \\
\hline 19 & $3233(20)$ & $3235(5)$ & $3232.45(10)$ & $4^{+}$ & & 3 \\
\hline 20 & $3283(20)$ & $3287(5)$ & $3284.89(17)$ & & $2^{-}, 4^{+}$ & $2^{+}$ \\
\hline 21 & $3349(20)$ & $3342(5)$ & $3352.01(12)$ & $2^{+}$ & $3^{-}, 4^{+}$ & $2^{+}$ \\
\hline \multirow[t]{2}{*}{22} & $3428(20)$ & $3430(5)$ & $3433.58(25)$ & $4^{+}, 5^{-}$ & $4^{+}$ & $4^{+}$ \\
\hline & & & $3441.97(17)$ & & & $4^{+}$ \\
\hline 23 & $3464(20)$ & $3474(5)$ & $3472.18(29)$ & & $2^{+}, 4^{+}, 5^{-}$ & $2^{+}$ \\
\hline 24 & $3542(20)$ & $3549(5)$ & $3540.80(14)$ & & 3 & 3 \\
\hline 25 & $3601(20)$ & $3597(5)$ & $3610.46(24)$ & $\left(3^{-}\right)$ & $2^{+}$ & 2,3 \\
\hline
\end{tabular}

Table 7.4: Energy, spin and parity assignments of the ${ }^{96} \mathrm{Mo}\left(p, p^{\prime}\right)$ experiment and comparison to other experiments.

and a $4^{+}$state at $2219.35 \mathrm{keV}$. The $2^{+}$state is assumed to be the one-phonon mixed-symmetry state. Due to the proximity of those states to the strongly excited $3^{-}$state it was not possible to resolve them in the present experiment.

state at $2428 \mathrm{keV}$.

There is evidence for a state at $2428 \mathrm{keV}$. Due to its proximity to the $3_{1}^{-}$state no meaningful angular distribution of the cross section could be extracted. Refs. [Fre87, Les07] assign $J^{\pi}=2^{+}$ to this state.

$4_{3}^{+}$state at $2481 \mathrm{keV}$.

The measured spin, parity and energy agree with a $4^{+}$state measured in Refs. [Fre87, Les07]. 


\section{$\left(2^{+}\right)$state at $2539 \mathrm{keV}$.}

The position of the first maximum indicates $J^{\pi}=2^{+}$. However at scattering angles larger than $15^{\circ}$ degrees the calculation fails to describe the data. The deviations might be explained by taking into account contributions of the $3^{-}$state of ${ }^{94} \mathrm{Mo}$ which is at an energy of $2533.87 \mathrm{keV}$. Ref. [Les07] makes a $J^{\pi}=\left(2^{+}, 3^{+}\right)$assignment. Since this state that was excited in the present experiment $J^{\pi}=2^{+}$is likely.

$4^{+}$state at $2626 \mathrm{keV}$.

In agreement with Refs. [Fre87, Les07] spin and parity of $J^{\pi}=4^{+}$is assigned to this state.

$\left(2^{+}\right)$state at $2700 \mathrm{keV}$.

The position of the first maximum gives evidence of a $2^{+}$state. Since the description at larger scattering angles is poor only a tentative $J^{\pi}=2^{+}$assignment was made. Ref. [Fre87] does not report this state, while Ref. [Les07] also finds a $2^{+}$state at an energy of $2700.08 \mathrm{keV}$.

$6^{+}$state at $2739 \mathrm{keV}$.

The angular distribution of the cross section is nicely described assuming spin and parity $J^{\pi}=$ $6^{+}$. Ref. [Les07] also reports of a $6^{+}$state but at a slightly higher energy of $2755.12 \mathrm{keV}$. At $2734.61 \mathrm{keV}$ it finds evidence of a $4^{+}$state. Ref. [Fre87] reports of a tentative $5^{-}$state at an energy of $2734 \mathrm{keV}$.

states at $2809 \mathrm{keV}, 2875 \mathrm{keV}$ and $3020 \mathrm{keV}$.

Three states were measured at energies of $2809 \mathrm{keV}, 2875 \mathrm{keV}$ and $3020 \mathrm{keV}$. For none of them a meaningful angular distribution could be extracted. Refs. [Fre87, Les07] finds states at approximately the same energies. Only Ref. [Les07] makes clear spin and parity assignments for the states at $2818.53 \mathrm{keV}\left(J^{\pi}=4^{+}\right)$and at $2986.79 \mathrm{keV}\left(\mathrm{J}^{\pi}=2^{+}\right)$.

$5^{-}$state at $3018 \mathrm{keV}$.

In disagreement with Refs. [Fre87, Les07] the cross section is well described assuming $J^{\pi}=5^{-}$. Ref. [Fre87] reports a $4^{+}$state at the same energy, while Ref. [Les07] finds a $2^{+}$state at an energy of $3024.47 \mathrm{keV}$.

$2^{+}$state at $3137 \mathrm{keV}$.

The angular distribution of the cross section clearly indicates a $2^{+}$state. Ref. [Les07] finds evidence for a $2^{+}$state very close in energy at $3134.50 \mathrm{keV}$. Ref. [Fre87] also sees a state at $3140 \mathrm{keV}$ but does not give spin and parity.

$3^{-}$state at $3179 \mathrm{keV}$.

$J^{\pi}=3^{-}$of Refs. [Fre87, Les07] was confirmed by the angular distribution data of the cross section.

$4^{+}$state at $3233 \mathrm{keV}$.

The angular distribution data points to a $4^{+}$state. Refs. [Fre87, Les07] find states at similar energies. However Ref. [Les07] makes a $J=3$ assignment, while Ref. [Fre87] does not give a spin or parity.

state at $3283 \mathrm{keV}$.

No conclusion about spin and parity could be drawn for this state. Ref. [Fre87] limits the possible quantum number to $2^{-}$and $4^{+}$. In contrast Ref. [Les07] finds a $2^{+}$state at an energy of $3284.89 \mathrm{keV}$.

$2^{+}$state at $3349 \mathrm{keV}$.

The cross section data gives clear evidence of a $2^{+}$state which is in agreement with the findings of Ref. [Les07]. In contrast to this Ref. [Fre87] assigns $\mathrm{J}^{\pi}=3^{-}, 4^{+}$to a state at $3342 \mathrm{keV}$.

$4^{+}, 5^{-}$state at $3428 \mathrm{keV}$.

The angular distribution points to a $4^{+}$state or a $5^{-}$state. However no firm assignment could 

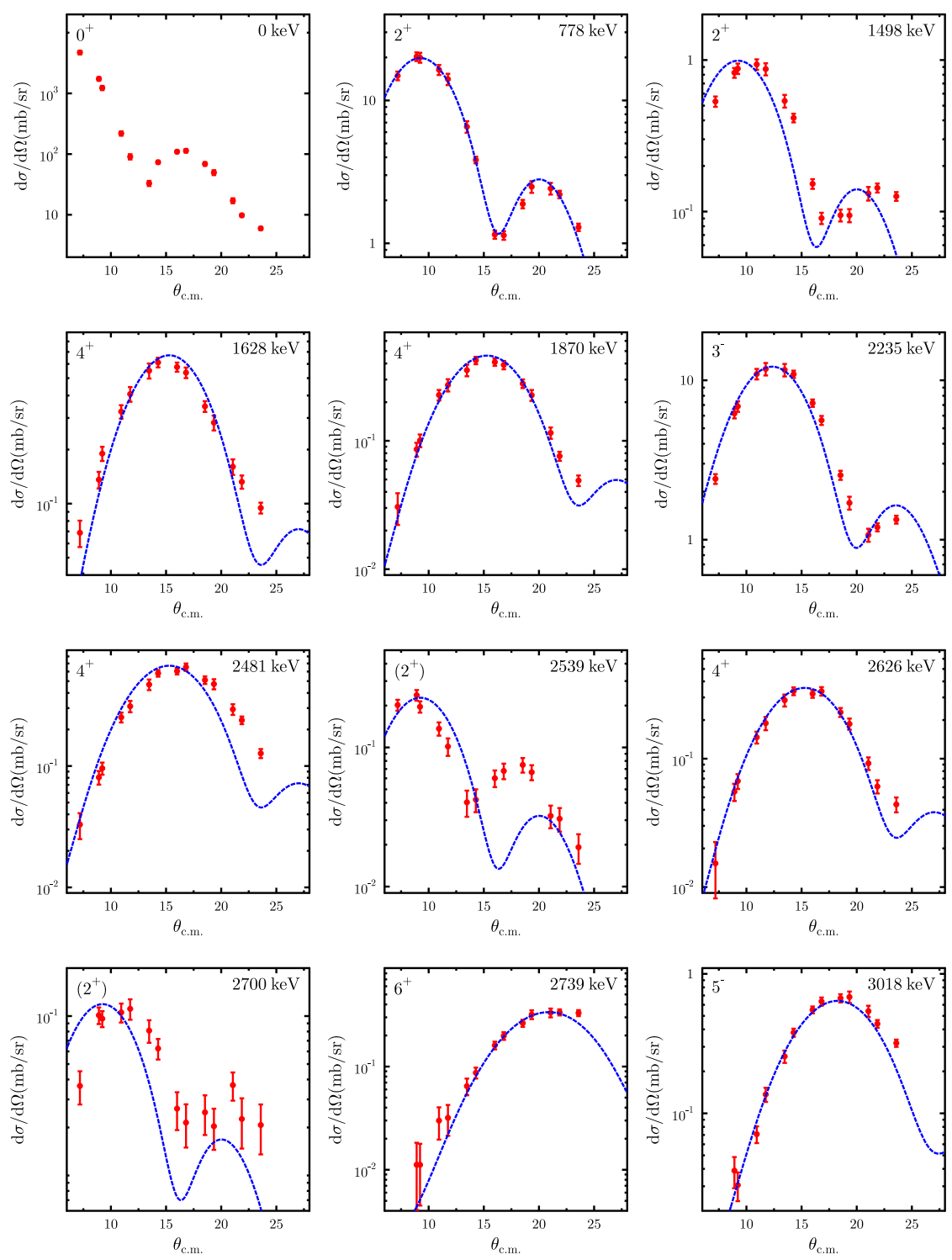

Figure 7.15: Same as Fig. 7.10 for ${ }^{96} \mathrm{Mo}\left(p, p^{\prime}\right)$.

be made. Ref. [Fre87] assigns $J^{\pi}=4^{+}$to this state, while Ref. [Fre87] finds two $4^{+}$states at energies of $3433.58 \mathrm{keV}$ and $3441.97 \mathrm{keV}$.

states at $3464 \mathrm{keV}$ and $3542 \mathrm{keV}$.

No conclusion about spin and parity could be drawn from the angular distributions of those states. Ref. [Fre87] as well as Ref. [Les07] finds states at similar energies.

tentative $3^{-}$state at $3601 \mathrm{keV}$.

Because of deviations at scattering angles larger than $15^{\circ}$ only a tentative $J^{\pi}=3^{-}$was assigned to the state. This is consistent with the findings of Ref. [Les07] which reports a $J=2,3$ state at $3610.46 \mathrm{keV}$. But in disagreement with Ref. [Fre87] which finds a $2^{+}$state at $3597 \mathrm{keV}$. 

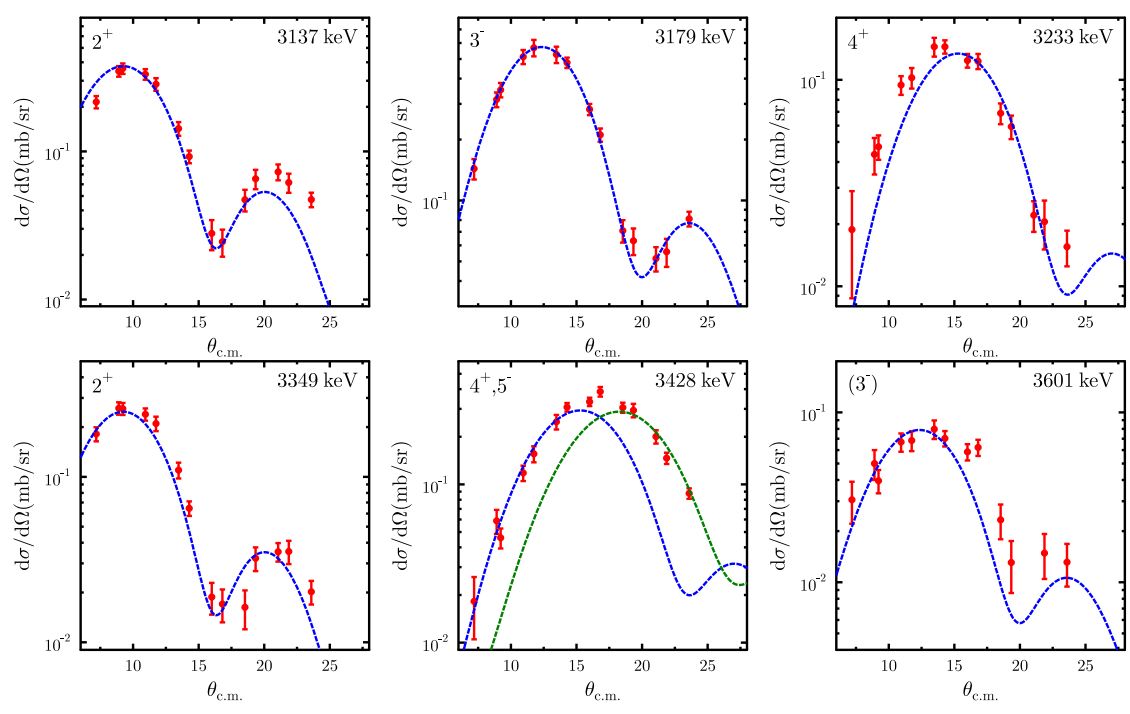

Figure 7.16: Same as Fig. 7.15

\subsubsection{Results of the ${ }^{70} \mathrm{Zn}\left(p, p^{\prime}\right)$ experiment}

$2_{1}^{+}$at $875 \mathrm{keV}$.

The cross section is described well using a collective model and a phenomenological optical potential assuming a spin of 2 and positive parity. This is in agreement with the results of Refs. [Jab87, Hud72, Mue09, Tu104].

\section{$2_{2}^{+}$and $4_{1}^{+}$states at 1761 and $1787 \mathrm{keV}$.}

The well known $2^{+}$state forms a doublet with the $4_{1}^{+}$state at $1787 \mathrm{keV}$ which cannot be resolved. For this reason the position of the centroids were fixed in the fit using the known energies of Ref. [Tu104], i.e. no energy could be determined for the $4_{1}^{+}$state. However at low scattering angles the contribution of the $4_{1}^{+}$state to the doublet was negligible and the energy of the $2_{2}^{+}$state was measured. Meaningful angular distributions of the cross sections were measured in both cases. The spin and parity assignments of Refs. [Jab87, Hud72, Mue09, Tul04] are confirmed. A clear shift of the features of the cross section to higher scattering angles compared to the model calculation can be seen for the $2_{2}^{+}$state. This is most likely due to a smaller matter transition radius than predicted by the collective model. The $2_{2}^{+}$state has a large twophonon component in its wave function as indicated by the large $B(E 2)$-value to the $2_{1}^{+}$state of 22.5(39) W.u. [Mue09]. However the cross section shape points two a one-step excitation of the state indicating also a sizable one-phonon component.

\section{$2_{3}^{+}$state at $1958 \mathrm{keV}$.}

This state is assumed to be the one-phonon quadrupole mixed-symmetry state because of its large $B(M 1)$-value of $0.231(27) \mu_{N}^{2}$ to the $2_{1}^{+}$state. Spin and parity assignments are in agreement with Refs. [Jab87, Hud72, Mue09, Tul04]. As for all $2^{+}$states the features of the cross section is shifted to higher scattering angles with respect the $2_{1}^{+}$state and the model calculation. The $B(E 2)$-value is at least a factor of $\sim 330$ smaller than the $B(E 2)$-value of the $2_{1}^{+}$state. This is in contrast to to the proton scattering cross section were the maxima differ only by a 


\begin{tabular}{|c|c|c|c|c|c|c|c|c|}
\hline No & $\begin{array}{c}E_{x} \\
(\mathrm{keV})^{1}\end{array}$ & $\begin{array}{c}E_{x} \\
(\mathrm{keV})^{2}\end{array}$ & $\begin{array}{c}E_{x} \\
(\mathrm{keV})^{3}\end{array}$ & $\begin{array}{c}x \\
(\mathrm{keV})^{4}\end{array}$ & $J^{\pi 1}$ & $J^{\pi 2}$ & $J^{\pi 3}$ & $J^{\pi 4}$ \\
\hline 1 & 0 & 0 & 0 & 0 & $0^{+}$ & $0^{+}$ & $0^{+}$ & $0^{+}$ \\
\hline 2 & $875(20)$ & $886(5)$ & $884(10)$ & $884.89(9)$ & $2^{+}$ & $2^{+}$ & $2^{+}$ & $2^{+}$ \\
\hline 3 & 1761(10) & 1764(5) & $1767 ?(10)$ & $1759.30(10)$ & $2^{+}$ & $2^{+}$ & & $2^{+}$ \\
\hline 4 & 1787 & 1790(5) & 1787(10) & $1786.62(14)$ & $4^{+}$ & $4^{+}$ & $4^{+}$ & $4^{+}$ \\
\hline 5 & $1958(10)$ & $1960(5)$ & 1955(10) & 1957.15(13) & $2^{+}$ & $2^{+}$ & $2^{+}$ & $2^{+}$ \\
\hline 6 & $2667(10)$ & $2665(5)$ & 2661(10) & 2659.3 & $2^{+}$ & $2^{+}$ & $2^{+}$ & $2^{+}$ \\
\hline 7 & $2695(10)$ & 2694(5) & $2690 ?(10)$ & $2693.24(16)$ & $4^{+}$ & $(3-5)^{+}$ & & $4^{+}$ \\
\hline 8 & $2865(10)$ & 2863(5) & 2856(10) & $2859.59(12)$ & $3^{-}$ & $3^{-}$ & $3^{-}$ & $3^{-}$ \\
\hline 5 & $2930(20)$ & 2954(5) & & 2949.3 & & & & $2^{+}, 3^{+}$ \\
\hline 9 & 2985(10) & 2975(5) & 2971(10) & 2978.37(23) & $4^{+}$ & $4^{+}$ & $4^{+}$ & $4^{+}$ \\
\hline 10 & $3044(10)$ & $3042(5)$ & $3031(10)$ & $\begin{array}{c}3038.30(16) \\
3038 ?\end{array}$ & $2^{+}+5^{-}$ & $5^{-}$ & $\left(5^{-}\right)$ & $5^{-}$ \\
\hline 11 & $3078(20)$ & & & $\begin{array}{l}3038.2 \\
3083.1\end{array}$ & & & & $\begin{array}{l}2,3 \\
(1)\end{array}$ \\
\hline 12 & $3133(20)$ & & & 3136.9 & & & & \\
\hline 13 & $3232(10)$ & $3235(5)$ & $3232(10)$ & $3246.81(12)$ & $\left(3^{-}\right)$ & $4^{+}$ & $\left(4^{+}\right)$ & $\left(4^{-}\right)$ \\
\hline 14 & $3364(10)$ & & $3340(10)$ & $3341.8(4)$ & $3^{-}$ & & $\left(3^{-}\right)$ & $3^{-}$ \\
\hline 15 & $3432(20)$ & $3419(5)$ & $3423(10)$ & 3422.0 & $2^{+}$ & $3^{-}$ & $\left(3^{-}\right)$ & $2^{+}$ \\
\hline 16 & $\sim 3500$ & $3464(5)$ & $3458(10)$ & 3454.3 & $4^{+}+5^{-}$ & $4^{+}$ & $4^{+}$ & $1,2,3$ \\
\hline & & $3506(5)$ & 3502(10) & 3520.7 & & $5^{-}$ & $5^{-}$ & $\leq 4$ \\
\hline 17 & $3646(10)$ & $3635(5)$ & 3631(10) & $3635.16(23)$ & $\left(3^{-}\right)$ & $2^{+}$ & $2^{+}$ & $2^{+}$ \\
\hline 18 & $3720(10)$ & $3712(5)$ & 3707(10) & $3710.6(6)$ & $2^{+}$ & $2^{+}$ & $2^{+}$ & $2^{+}$ \\
\hline 19 & $3741(20)$ & $3750(5)$ & $3746(10)$ & & & & & \\
\hline 20 & $3803(20)$ & $3813(5)$ & $3802(10)$ & & & & & \\
\hline 21 & $3844(10)$ & $3844(5)$ & $3839(10)$ & $3848.3(7)$ & & & $1^{-}$ & \\
\hline 22 & $3908(10)$ & $3888(5)$ & $3914(10)$ & $3904.9(5)$ & & $(3-5)^{+}$ & & \\
\hline 23 & $3989(10)$ & & $3999(10)$ & & $4^{+}+5^{-}$ & & $2^{+}$ & \\
\hline 24 & $4052(10)$ & $4066(10)$ & $4060(10)$ & 4061.42(19) & $2^{+}$ & $4^{+}$ & $4^{+}$ & $4^{+}$ \\
\hline 25 & $4107(20)$ & $4136(10)$ & $4140(10)$ & & & $(1-3)^{+}$ & & \\
\hline 26 & $4166(10)$ & $4172(10)$ & & & $4^{+}$ & $5^{-}$ & & \\
\hline 27 & $4210(10)$ & & & & $5^{-}$ & & & \\
\hline 28 & $4259(20)$ & $4284(10)$ & 4297(10) & $4264.6(8)$ & & $2^{+}$ & $2^{+}$ & \\
\hline 29 & 4301(10) & $4309(10)$ & & 4309.03(21) & $3^{-}$ & & & \\
\hline 30 & $4332(20)$ & & & & & & & \\
\hline 31 & 4395(20) & $4367(10)$ & & & & $(3-5)^{+}$ & & \\
\hline 32 & $4449(20)$ & $4444(10)$ & & & & $(3-5)^{+}$ & & \\
\hline 33 & $4473(10)$ & & & $4463.80(20)$ & $2^{+}$ & & & \\
\hline 34 & $4515(20)$ & & & $4514.4(3)$ & & & & \\
\hline 35 & $4582(10)$ & & & $4588.4(4)$ & $\left(3^{-}\right)$ & & & \\
\hline 36 & $4616(20)$ & & & & & & & \\
\hline 37 & $4660(10)$ & & & & $\left(3^{-}\right)$ & & & \\
\hline 38 & $4720(20)$ & & & $4710.1(6)$ & & & & \\
\hline
\end{tabular}

1 this work

2 Ref. [Jab87]

${ }^{3}$ Ref. [Hud72]

${ }^{4}$ Ref. [Mue09] and $\beta$-decay data of Ref. [Tul04]

Table 7.5: Energy, spin and parity assignments of the ${ }^{70} \mathrm{Zn}\left(p, p^{\prime}\right)$ experiment and comparison to other experiments.

factor of $\sim 39$. This points to a dominant neutron component in the wave function of the $2_{3}^{+}$ state.

states at 2340 and $2410 \mathrm{keV}$.

These states have not been seen in Refs. [Hud72, Mue09, Tul04] but Ref. [Jab87] sees a $2^{+}$state at 2375 (5) $\mathrm{keV}$. It was not possible to extract a meaningful angular distribution for the $2410 \mathrm{keV}$ state but the $2340 \mathrm{keV}$ state is clearly a $2^{+}$state. Due to the proximity of those two states to a $2^{+}$state and a $4^{+}$state in ${ }^{68} \mathrm{Zn}(2338.45(5) \mathrm{keV}$ and $2417.40(6) \mathrm{keV}$, respectively) the states were interpreted as excitations of ${ }^{68} \mathrm{Zn}$. 


\section{$2_{4}^{+}$state at $2667 \mathrm{keV}$.}

The measured energy, spin and parity agree with the properties of a $2^{+}$state seen in Refs. [Jab87,Hud72,Mue09]. The centroid of the peak was fixed in most of the fits due to its proximity to a much stronger excited $4^{+}$state.

$4_{2}^{+}$and $3_{1}^{-}$states at 2695 and $2865 \mathrm{keV}$.

Both states were also seen in Refs. [Jab87, Hud72, Mue09, Tul04], while Refs. [Jab87, Hud72] doesn't make a clear spin assignment for the $4_{2}^{+}$state. In the present experiment both angular distributions are well described assuming a spin and parity of $4^{+}$and $3^{-}$, respectively.

state at $2930 \mathrm{keV}$.

No conclusions about spin and parity could be drawn from the angular distribution of this state. Refs. [Tul04, Mue09] limit the possible quantum number to $2^{+}$and $3^{+}$. Since a $J^{\pi}=3^{+}$is only weakly excited in the present reaction, $J^{\pi}=2^{+}$are the most likely quantum numbers.

$4_{3}^{+}$state at $2985 \mathrm{keV}$.

In agreement with Refs. [Jab87, Hud72, Mue09, Tul04] this state is clearly identified as a $4^{+}$ state.

$2^{+}+5_{1}^{-}$doublet at $3044 \mathrm{keV}$.

Although Refs. [Jab87, Tul04] has identified this state as a $5^{-}$state, the angular distribution is best described assuming a doublet of a $2^{+}$and a $5^{-}$state. This is in agreement with Ref. [Mue09] which found a state at $3038.2 \mathrm{keV}$ and an other state at the same energy with $J=(2,3)$. No parity is given for the second state. Considering this experiment $J^{\pi}=2^{+}$can be assigned to the second state.

states at 3078 and $3133 \mathrm{keV}$.

No spin could be assigned to these states. Only Ref. [Mue09] sees two states at the same energy, but gives only a tentative spin assignment of $J=1$ for the level at $3078 \mathrm{keV}$. A spin of $J=1$ is not very likely because the state is excited in this experiment.

$\left(3^{-}\right)$state at $3232 \mathrm{keV}$.

The position of the maximum points to $J^{\pi}=3^{-}$. However there are clear deviations from the the expected angular distribution of a $3^{-}$state. For this reason only a tentative $3^{-}$assignment hwas made. Ref. [Jab87] assigns quantum $J^{\pi}=4^{+}$to the state, Ref. [Hud72] does the same tentatively. Ref. [Mue09] reports two close lying states at $3224.3 \mathrm{keV}$ and $3247.0 \mathrm{keV}$ with quantum numbers $J=1$ and $J=\left(4^{-}\right)$, respectively. The nature of this state remains unclear.

$3_{2}^{-}$state at $3364 \mathrm{keV}$.

The angular distributions of the cross section clearly indicates a $3^{-}$state. This strongly excited state was not observed in Ref. [Jab87]. Ref. [Tul04] finds evidence for a $3^{-}$state at an energy of $3341.6 \mathrm{keV}$. It is not clear if the state seen at $3364 \mathrm{keV}$ corresponds to this state or rather to a state at $3357.4 \mathrm{keV}$ with possible quantum numbers $J=1,2,3$.

$2_{5}^{+}$state at $3432 \mathrm{keV}$.

The good agreement between model prediction and experimental data give clear evidence for $J^{\pi}=2^{+}$. This is in agreement with Ref. [Mue09]. On the other hand Ref. [Jab87] reports $J^{\pi}=3^{-}$.

doublet at $3500 \mathrm{keV}$.

This state is a clear doublet. The measured angular distributions is best described assuming $J^{\pi}=4^{+}$and $J^{\pi}=5^{-}$. From the data it is clear that the $4^{+}$state is at a higher energies than the $5^{-}$state. Refs. [Jab87, Hud72] find a $5^{-}$and a $4^{+}$state close to $3500 \mathrm{keV}$. However in these two experiments the $5^{-}$is at a higher energy, i.e. there is a disagreement between those two experiments and the present experiment. 
$\left(3^{-}\right)$at $3646 \mathrm{keV}$.

In disagreement with Refs. [Jab87,Hud72, Tul04] the position of the maximum gives evidence of $\mathrm{J}^{\pi}=3^{-}$. Because of some deviations at larger scattering angles only a tentative $3^{-}$assignment was made. However there is no hint for a $2^{+}$state as seen in other experiments.

$2_{6}^{+}$at $3720 \mathrm{keV}$.

$J^{\pi}=2^{+}$of Refs. [Jab87, Hud72, Tul04] was confirmed by the angular distribution data of the cross section.

states at 3741, 3803, 3844 and $3908 \mathrm{keV}$.

No conclusions about spin and parity could be drawn for those states. However in Refs. [Jab87, Hud72, Tul04] states were found at similar energies. Ref. [Hud72] makes a $J^{\pi}=1^{-}$assignment for the state at $3844 \mathrm{keV}$. This is not in agreement with the present experiment because a $1^{-}$ would only be very weakly excited.
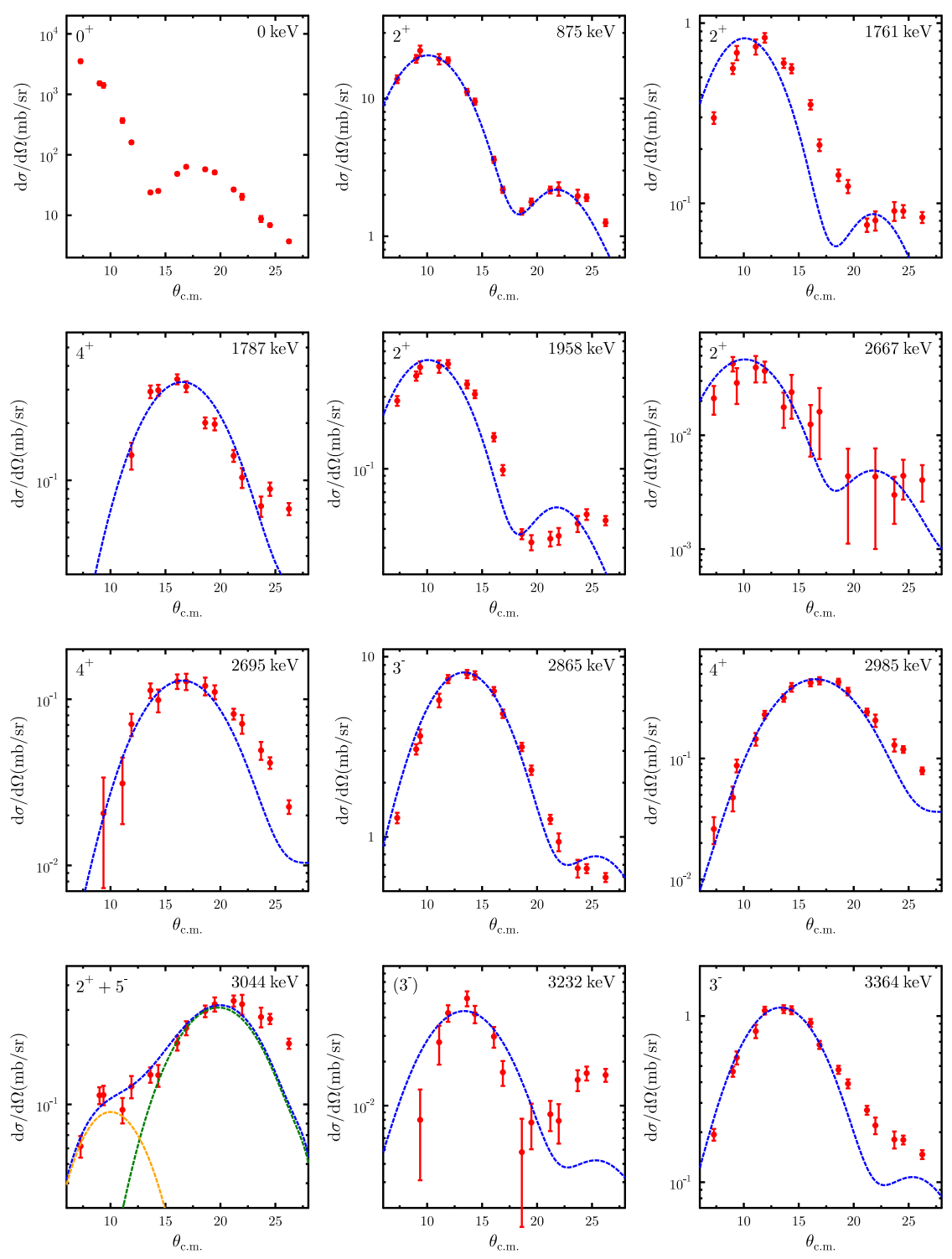

Figure 7.17: Same as Fig. 7.10 for ${ }^{70} \mathrm{Zn}\left(p, p^{\prime}\right)$. 

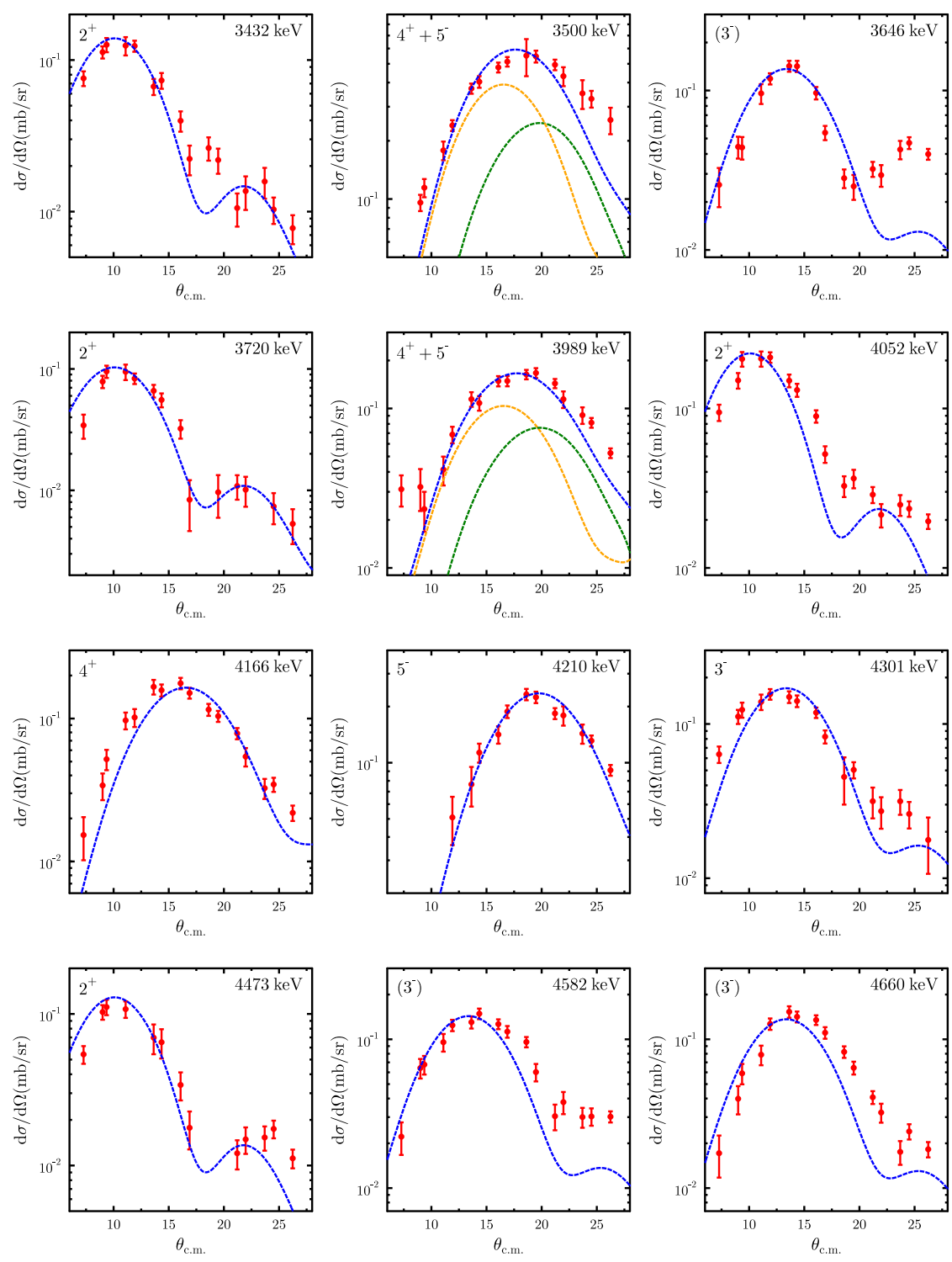

Figure 7.18: Same as Fig. 7.17

\section{$4^{+}+5^{-}$doublet at $3989 \mathrm{keV}$.}

This state is a doublet. Its angular distribution is best described assuming a doublet of $J^{\pi}=4^{+}$ and $J^{\pi}=5^{-}$states. It was only measured in Ref. [Hud72] where $\mathrm{J}^{\pi}=2^{+}$is assigned.

$2_{7}^{+}$at $4052 \mathrm{keV}$.

In contrast to Refs. [Jab87, Hud72, Tul04] the angular distribution gives evidence of a $2^{+}$state. All other experiment assign $J^{\pi}=4^{+}$to this state.

Up to this point it was tried to make a one-to-one correspondence between the states seen in the various experiments. This is very difficult for states above $\sim 4 \mathrm{MeV}$ because of the high level density, i.e. the comparison given in Tab. 7.5 should be treated with care. There are usually several states in the other references which are close to the states seen in this experiment.

Since the energy resolution was $32-52 \mathrm{keV}$ it was difficult to fit the peaks above an excitation energy of $\sim 4 \mathrm{MeV}$. Almost all of them overlap with other peaks which makes it difficult to 
determine cross sections and angular distributions. However it was still possible to assign some spin and parity quantum numbers as shown in Tab. 7.5. 


\section{Interpretation}

For the neighboring nuclei ${ }^{92,94} \mathrm{Zr}$ and ${ }^{94} \mathrm{Mo}$ a large body of nuclear structure observables of low-lying states is available including energies, transition strengths to the ground state as well as between excited states and g-factors. The proton scattering cross sections measured in this thesis and the electron scattering data of Refs. [Bur07, Sch13, Wal10] complete this data set. They provide complementary information on the structure of excited states. Electromagnetic $B(E 2)$-values to the ground state are only sensitive to the proton part of the wave functions and are integral quantities of the transition densities. On the other hand proton and electron scattering also probe the radial structures of the excitations. When the momentum transfers are low, both reactions are mainly sensitive to the surface region of the nucleus and in particular to the transition radii. In addition, proton scattering gives information on the absolute neutron transition matrix elements of excited states to the ground state.

This large and complete data set consisting of observables which are sensitive to different parts of the wave functions provides a stringent and detailed test for nuclear structure models. In this work the model of choice was the Quasi-Particle Photon Model (QPM) which is outlined in Sect. 5.3. If the test of the QPM is successful one can go one step ahead and investigate the model wave functions in more detail in order to understand why the experimental data exhibit several interesting features. To be more specific the outline and objectives of the theoretical analysis presented in the next sections are the following.

- The experimental data is used to test the ability of the QPM to describe low-lying natural parity states in nuclei near closed shells in the $N=50$ and $Z=40$ mass region. This is done in Sect. 8.1 by comparing the QPM predictions to experimental data - separately for the multipolarities $\mathrm{J}^{\pi}=2^{+}, 3^{-}, 4^{+}$and $5^{-}$- for each and every available observable. Shortcomings of the model are pointed out.

- Recently Refs. [Cas13, Sch10] discussed octupole and hexadecapole one-phonon mixedsymmetry in ${ }^{92} \mathrm{Zr}$ and ${ }^{94} \mathrm{Mo}$. In the present work the $3^{-}$and $4^{+}$states were studied in the QPM and it was tried to verify the claims of Refs. [Cas13, Sch10] in Sect. 8.1. For this purpose the proton and electron scattering cross sections are useful observables because they are related to the $B(E 3)$ - and $B(E 4)$-transition strengths to the ground state which were not available to the authors of Refs. [Cas13, Sch10].

- Also in Sect. 8.1 the basic degrees of freedom are identified which are important for the description of the low-lying states in the investigated nuclei. A simple picture is developed to obtain an intuitive understanding of the formation mechanism of these building blocks and their mutual interplay.

- In even-even nuclei the $2_{1}^{+}$state has an enhanced $B(E 2)$-value to the ground state. This feature is attributed to contributions from 2qp-states outside the valence space. This is usually referred to as core polarization. Due to its large model space the QPM allows to study this phenomenon microscopically which is done in Sect. 8.2 for the $2^{+}$and $4^{+}$states. 
- A special emphasis of this work is on one-phonon quadrupole mixed-symmetry states. In the author's master thesis [Wal10] a new method was introduced to confirm the mixedsymmetric character of the $2_{2}^{+}$state in ${ }^{92} \mathrm{Zr}$ based on the shapes of proton and electron scattering cross sections. In Sect. 8.3 the same method is applied to the nuclei ${ }^{94} \mathrm{Mo}$ and ${ }^{94} \mathrm{Zr}$.

\subsection{Comparison of QPM calculations to experiment}

\subsubsection{Ground state properties}

In the QPM the QRPA-phonons are created by acting with the Q-phonon operator of Eq. 5.30 on the ground state. Therefore it is important to verify the predictions of the QPM for the ground state wave functions compositions with a comparison to experimental observables of the ground state. Figure 8.1 compares the root-mean-square radii of the proton density distributions (sometimes called charge density distributions) calculated in the QPM to experimental values obtained from elastic electron scattering for the nuclei ${ }^{92,94} \mathrm{Zr}$ and ${ }^{94} \mathrm{Mo}$ [Vri87]. The QPM overestimates slightly the proton radius of ${ }^{92} \mathrm{Zr}$ by $0.03 \mathrm{fm}$, while the predicted radii of ${ }^{94} \mathrm{Zr}$ and ${ }^{94} \mathrm{Mo}$ are in excellent agreement with data within the error bars. The quality of the description is good pointing to the fact that the QPM correctly describes the charge density distributions of the ground states.

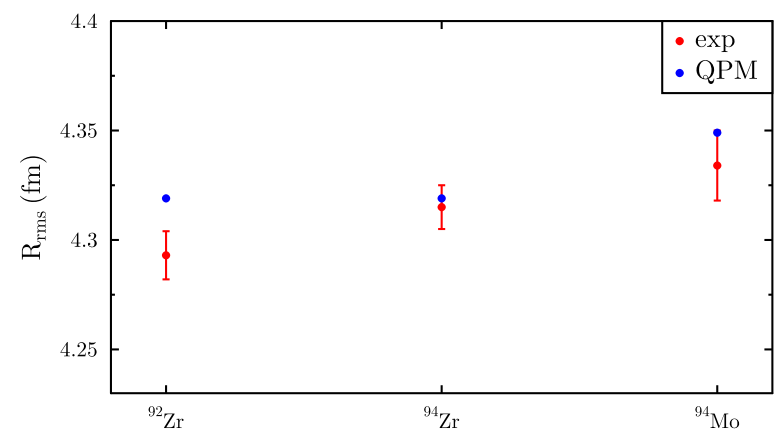

Figure 8.1: Comparison of the calculated and measured root-mean-square radii of the charge distributions.

The proton scattering experiments presented in Chap. 7 also measured the elastic scattering cross sections which can be used to test the optical model obtained by folding the proton and neutron ground state densities of the QPM with the effective interaction described in Sect. 5.1.2. The comparison between theory and data is displayed in Fig. 8.2. Despite some deviations at small scattering angles in case of ${ }^{94}$ Mo the general description of the data is successful in terms of shapes as well as in magnitudes. It should be noted that the calculated cross sections are absolute and no changes were made on the effective nucleon-nucleus interaction. This is also true for all other proton scattering cross sections calculated in this work.

The good descriptions of the charge radii and the elastic proton scattering cross sections validate (to a certain degree) the QPM ground state wave functions as well as the effective nucleon-nucleus interaction. This sets all calculations of the properties of excited state presented in the following sections on a solid base. 

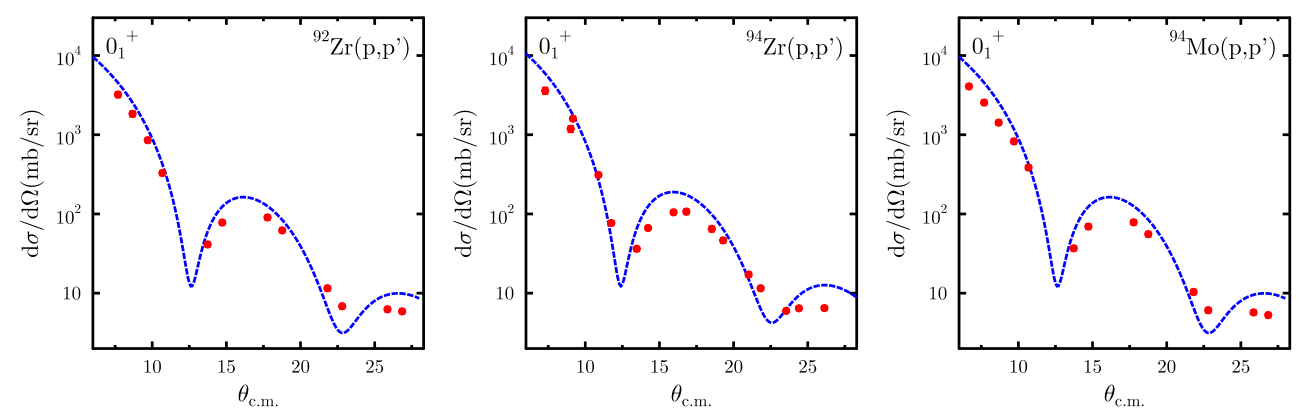

Figure 8.2: Comparison of the calculated and measured elastic proton scattering cross sections at an energy of $200 \mathrm{MeV}$.

\subsubsection{Quadrupole states}

Tab. 8.1 displays the two leading components of the wave functions of the first and second quadrupole QRPA-phonons for ${ }^{94} \mathrm{Mo}$ and ${ }^{92,94} \mathrm{Zr}$. The same two 2qp-states are important for each phonon: The neutron $\left(2 d_{5 / 2} \otimes 2 d_{5 / 2}\right)_{n}$ and the proton $\left(1 g_{9 / 2} \otimes 1 g_{9 / 2}\right)_{p}$ 2qp-states which are in-phase for the $\left[2_{1}^{+}\right]_{[R P A]}$-phonons and out-of-phase for the $\left[2_{2}^{+}\right]_{[R P A]}$-phonons (the forward amplitude $\psi$ determines the sign). According to the definition given in Sect. 5.3.3 the $\left[2_{2}^{+}\right]_{[R P A]^{-}}$ phonon can be considered as the mixed-symmetric phonon in all three nuclei.

\begin{tabular}{c|c|ccc|ccc}
\hline \hline & & \multicolumn{3}{|c|}{$\left(2 d_{5 / 2} \otimes 2 d_{5 / 2}\right)_{n}$} & \multicolumn{3}{|c}{$\left(1 g_{9 / 2} \otimes 1 g_{9 / 2}\right)_{p}$} \\
${ }^{\mathrm{A}} X$ & QRPA-phonons & $\psi$ & $\phi$ & $\%$ & $\psi$ & $\phi$ & $\%$ \\
\hline${ }^{94} \mathrm{Mo}$ & {$\left[2_{1}^{+}\right]_{[R P A]}$} & 1.06 & 0.20 & 54.64 & 0.87 & 0.30 & 32.95 \\
& {$\left[2_{2}^{+}\right]_{[R P A]}$} & -0.95 & 0.09 & 44.49 & 0.96 & 0.08 & 45.87 \\
\hline${ }^{92} \mathrm{Zr}$ & {$\left[2_{1}^{+}\right]_{[R P A]}$} & 1.20 & 0.16 & 70.98 & 0.51 & 0.19 & 11.03 \\
& {$\left[2_{2}^{+}\right]_{[R P A]}$} & -0.76 & 0.11 & 28.30 & 0.80 & 0.09 & 31.66 \\
\hline${ }^{94} \mathrm{Zr}$ & {$\left[2_{1}^{+}\right]_{[R P A]}$} & 1.27 & 0.20 & 78.20 & 0.42 & 0.20 & 6.93 \\
& {$\left[2_{2}^{+}\right]_{[R P A]}$} & -0.65 & 0.12 & 20.27 & 0.64 & 0.10 & 19.83 \\
\hline \hline
\end{tabular}

Table 8.1: Leading components of the wave functions of the $\left[2_{1}^{+}\right]_{[R P A]^{-}}$and $\left[2_{2}^{+}\right]_{[R P A]}-\mathrm{QRPA}-$ phonons for the nuclei ${ }^{94} \mathrm{Mo}$ and ${ }^{92,94} \mathrm{Zr}$. $\psi$ and $\phi$ are the forward and backward amplitudes defined in Eq. 5.30. The numbers in percentage are the contributions of the corresponding 2qp-states to the norm of the wave functions.

The formation mechanism of this structure can be understood in a simple two-state model. On the left-hand-side of Fig. 8.3 are shown two unperturbed 2qp-states. If one considers the proton-neutron residual interaction both 2-qp states mix and two states are formed, one where both 2-qp states are connected with a plus sign and one where both are connected with a minus. The former is the counterpart of the $\left[2_{1}^{+}\right]_{[R P A]}$-phonon and the latter is the counterpart of the $\left[2_{2}^{+}\right]_{[R P A]}$-phonon. The strength of the residual proton-neutron interaction and the energy difference of the unperturbed 2qp-states determine the degree of mixing i.e. the amplitudes $\alpha$ and $\beta$. In case of strong mixing $\alpha \approx \beta \approx \frac{1}{\sqrt{2}}$ and two optimally mixed states develop. The energy difference $\Delta \mathrm{E}$ of the pure $\left(2 \mathrm{~d}_{5 / 2} \otimes 2 \mathrm{~d}_{5 / 2}\right)_{n}$ - and $\left(1 g_{9 / 2} \otimes 1 g_{9 / 2}\right)_{p}$-2qp-states are $781 \mathrm{keV}$ in ${ }^{94} \mathrm{Mo}, 1255 \mathrm{keV}$ in ${ }^{92} \mathrm{Zr}$ and $1578 \mathrm{keV}$ in ${ }^{94} \mathrm{Zr}$. The strength of the residual interaction is 
comparable in all three nuclei [Pon14]. Therefore one would expect in the two-state model that the degree of mixing increases from ${ }^{94} \mathrm{Zr}$ to ${ }^{94} \mathrm{Mo}$. This is confirmed by the wave functions in Tab. 8.1. The $\left[2_{1}^{+}\right]_{[R P A]}$-phonons in ${ }^{94} \mathrm{Zr}$ and ${ }^{92} \mathrm{Zr}$ are neutron dominated ( ${ }^{92} \mathrm{Zr}$ to a lesser extend than ${ }^{94} \mathrm{Zr}$ ) while the $2_{1}^{+}$state in ${ }^{94} \mathrm{Mo}$ is close to the 'optimal case' . Nevertheless many other 2qp-states contribute to the wave functions in the QRPA calculations, somehow diminishing the two-state mixing picture. A large fraction of the wave function, however, can be understood in this simple scheme. It works particularly well in the three nuclei investigated here because the two important 2qp-states are slightly separated in energy from other 2qp-states. This can be seen in Fig. 5.4 which displays the 2qp-spectrum of ${ }^{92} \mathrm{Zr}$. Heyde and Sau introduced in Ref. [Hey86] a similar scheme proving that the formation of states with similar properties as the $\left[2_{1}^{+}\right]_{[R P A]^{-}}$and $\left[2_{2}^{+}\right]_{[R P A]}$-phonons are a general feature of two-component systems.

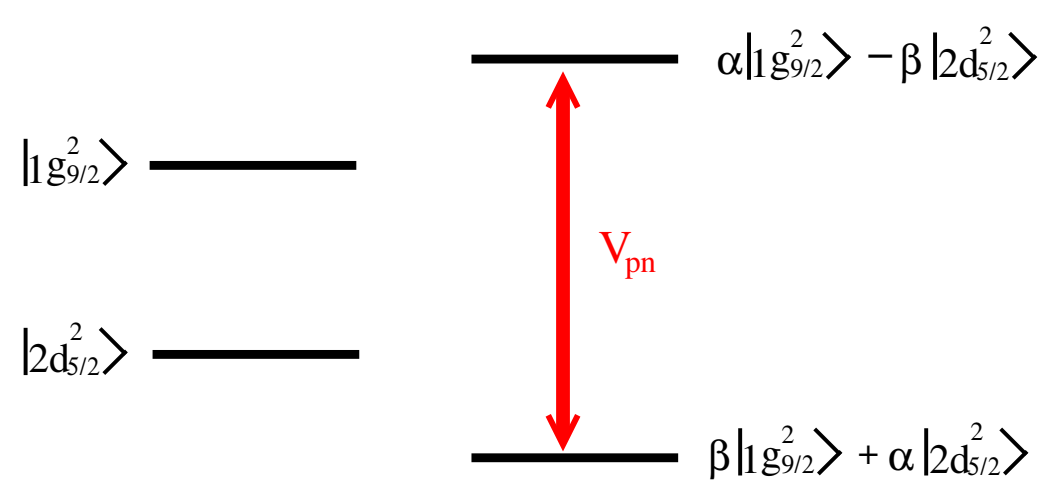

Figure 8.3: Simple two-state mixing model to explain the structure of the leading components of the QRPA-phonons in Tab. 8.1. The residual proton-neutron interaction mixes the two unperturbed 2qp-states forming two states were both 2qp-states are either connected with a plus sign or a minus sign.

As described in Sect. 5.3.1 the QPM takes into account the mixing between different QRPAphonons. The final wave functions of the three $2^{+}$states which are lowest in energy are shown in Tab. 8.2 in terms of QRPA-phonons. Clearly, in all three nuclei the $2_{1}^{+}$state is dominated by the $\left[2_{1}^{+}\right]_{[R P A]}$-phonon with a contribution of $\sim 90 \%$ to the wave function compositions. In ${ }^{92} \mathrm{Zr}$ the main fraction of the mixed-symmetric phonon is in the $2_{2}^{+}$state while it is in the $2_{3}^{+}$state in case of ${ }^{94} \mathrm{Mo}$. Each of these states are dominated by the $\left[2_{2}^{+}\right]_{[R P A]}$-phonon with contributions of $75 \%$ in ${ }^{94} \mathrm{Mo}$ and $91 \%$ in ${ }^{92} \mathrm{Zr}$. In ${ }^{94} \mathrm{Zr}$ the situation is entirely different. Here the unperturbed $\left[2_{2}^{+}\right]_{[R P A]}$-phonon is only a $\sim 10 \mathrm{keV}$ away from the $\left[2_{1}^{+} \otimes 2_{1}^{+}\right]_{[R P A]}$-two-phonon state. Therefore both phonon-states strongly mix. Two states are formed carrying large components of one- and two-phonon-components. In ${ }^{94} \mathrm{Mo}$ and ${ }^{92} \mathrm{Zr}$ the $\left[2_{1}^{+} \otimes 2_{1}^{+}\right]_{[R P A]}$ - and $\left[2_{2}^{+}\right]_{[R P A]}$-phonon-states are sufficiently separated in energy preventing such a strong mixing. Indeed two relatively pure twophonon states are formed with two-phonon components of $63 \%$ in ${ }^{94} \mathrm{Mo}$ and $54 \%$ in ${ }^{92} \mathrm{Zr}$. Some other phonons have a non-negligibile contribution like the $\left[2_{4}^{+}\right]_{[R P A]^{-}}$and $\left[2_{5}^{+}\right]_{[R P A]}$-phonons in ${ }^{92} \mathrm{Zr}$.

Which evidence can be found in the experimental data to support the QPM predictions? Tab. 8.3 compares the known electromagnetic transition strengths of the three lowest $2^{+}$ states in ${ }^{94} \mathrm{Mo}$ and ${ }^{92,94} \mathrm{Zr}$ to experimental values taken from Refs. [Fra03, Fra05, Elh08, Elh13a, Elh13b]. In ${ }^{94} \mathrm{Mo}$ and ${ }^{92} \mathrm{Zr}$ the collective E2-transitions of the $2_{1}^{+}$state to the ground state as well as the weakly collective $B(E 2)$-values of the mixed-symmetry states are reproduced by 


\begin{tabular}{|c|c|c|c|c|}
\hline \multirow[t]{2}{*}{${ }^{\mathrm{A}} X$} & \multirow[t]{2}{*}{ state } & \multicolumn{2}{|c|}{$E(\mathrm{keV})$} & \multirow[t]{2}{*}{ structure } \\
\hline & & Exp & QPM & \\
\hline \multirow{3}{*}{${ }^{94} \mathrm{Mo}$} & $2_{1}^{+}$ & 872 & 822 & $86 \%\left[2_{1}^{+}\right]_{[R P A]}$ \\
\hline & $2_{2}^{+}$ & 1864 & 1470 & $13 \%\left[2_{3}^{+}\right]_{[R P A]}+63 \%\left[2_{1}^{+} \otimes 2_{1}^{+}\right]_{[R P A]}$ \\
\hline & $2_{3}^{+}$ & 2067 & 1870 & $75 \%\left[2_{2}^{+}\right]_{[R P A]}-10 \%\left[2_{1}^{+} \otimes 4_{1}^{+}\right]_{[R P A]}$ \\
\hline \multirow{3}{*}{${ }^{92} \mathrm{Zr}$} & $2_{1}^{+}$ & 934 & 1025 & $91 \%\left[2_{1}^{+}\right]_{[R P A]}$ \\
\hline & $2_{2}^{+}$ & 1847 & 1983 & $91 \%\left[2_{2}^{+}\right][R P A]$ \\
\hline & $2_{3}^{+}$ & 2066 & 2043 & $17 \%\left[2_{4}^{+}\right]_{[R P A]}+13 \%\left[2_{5}^{+}\right]_{[R P A]}-54 \%\left[2_{1}^{+} \otimes 2_{1}^{+}\right]_{[R P A]}$ \\
\hline \multirow{3}{*}{${ }^{94} \mathrm{Zr}$} & $2_{1}^{+}$ & 919 & 856 & $93 \%\left[2_{1}^{+}\right]_{[R P A]}$ \\
\hline & $2_{2}^{+}$ & 1671 & 1961 & $35 \%\left[2_{2}^{+}\right]_{[R P A]}+44 \%\left[2_{1}^{+} \otimes 2_{1}^{+}\right]_{[R P A]}$ \\
\hline & $2_{3}^{+}$ & 2366 & 2090 & $49 \%\left[2_{2}^{+}\right]_{[R P A]}+31 \%\left[2_{1}^{+} \otimes 2_{1}^{+}\right]_{[R P A]}$ \\
\hline
\end{tabular}

Table 8.2: Leading components of the QPM wave functions of the three lowest quadrupole states for the nuclei ${ }^{94} \mathrm{Mo}$ and ${ }^{92,94} \mathrm{Zr}$.

the QPM calculation, although all four experimental values are slightly underestimated in the calculation.

For a state which has a dominant two-phonon component $\left[2_{1}^{+} \otimes 2_{1}^{+}\right]_{[R P A]}$ one expects a large $B(E 2)$-value to the $2_{1}^{+}$state of the order of $2 \cdot B\left(E 2: 2_{1}^{+} \rightarrow 0_{1}^{+}\right)$, a weak transition to the ground state and its energy is expected to be approximately at twice the energy of the $2_{1}^{+}$state. The experimental data for the $2_{2}^{+}$state in ${ }^{94} \mathrm{Mo}$ and the $2_{3}^{+}$state in ${ }^{92} \mathrm{Zr}$ is not in contradiction with this picture. But reliable information on the crucial transition to the $2_{1}^{+}$state is missing. In ${ }^{92} \mathrm{Zr}$ only an upper limit of $<14$ W.u. is known and the value in ${ }^{94} \mathrm{Mo}$ of $60_{-30}^{+20}$ W.u. has a very large error bar. It is important to obtain additional information about these two states in order to confirm their dominant two-phonon character. As will be discussed later in this section proton scattering cross sections provide this information and give indications for dominant two-phonon components in the wave functions. The QPM predictions for these states are in qualitative agreement with the data. The transitions to the ground state are underestimated in ${ }^{94} \mathrm{Mo}$ and overestimated in ${ }^{92} \mathrm{Zr}$. The strengths of these transitions are determined by small admixtures of one-phonon components in the wave functions like the $\left[2_{3}^{+}\right]_{[R P A]}$-phonon in ${ }^{94} \mathrm{Mo}$. One can not expect the QPM to describe such details of the wave functions accurately. In general it is promising that the QPM is able to describe the trend of the $B(E 2)$-values to the ground state i.e. a collective value for the $2_{1}^{+}$state, a weakly collective transition for the $2_{\mathrm{ms}}^{+}$state and a very weak transition for the two-phonon state.

Tab. 8.2 compares the experimental energies to the QPM-results. For ${ }^{94} \mathrm{Mo}$ and ${ }^{92} \mathrm{Zr}$ the QPM gives a good description of the data. The only serious discrepancy is the energy of the $2_{2}^{+}$state in ${ }^{94} \mathrm{Mo}$ which is $\sim 400 \mathrm{keV}$ lower than the experimental value. The unperturbed energy of its main component $\left[2_{1}^{+} \otimes 2_{1}^{+}\right]_{[R P A]}$ is at $2618 \mathrm{keV}$. It is pushed down in energy by the interaction with three-phonon states at higher energies. The very low energy of the final state of $1470 \mathrm{keV}$ suggests that the strength of the interaction between this two-phonon state and the three-phonon states is overestimated.

In ${ }^{94} \mathrm{Zr}$ the QPM calculations exhibit additional problems for the energies as well as for the electromagnetic transition strengths. Due to proximity of the unperturbed $\left[2_{1}^{+} \otimes 2_{1}^{+}\right]_{[R P A]}$ and $\left[2_{2}^{+}\right]_{[R P A]}$ energies the second and the third $2^{+}$states have large two-phonon components and therefore enhanced $B(E 2)$-values to the $2_{1}^{+}$state. This is not seen in experiment. The E2- 


\begin{tabular}{|c|c|c|c|c|c|c|}
\hline \multirow[b]{2}{*}{${ }^{\mathrm{A}} X$} & \multirow[b]{2}{*}{$J_{i} \rightarrow J_{f}$} & \multicolumn{3}{|c|}{$B(E 2)($ W.u. $)$} & \multicolumn{2}{|c|}{$B(M 1)\left(\mu_{N}^{2}\right)$} \\
\hline & & Exp & $\mathrm{QPM}_{\mathrm{p}}$ & $\mathrm{QPM}_{\mathrm{n}}$ & exp & QPM \\
\hline \multirow{5}{*}{${ }^{94} \mathrm{Mo}$} & $2_{1}^{+} \rightarrow 0_{1}^{+}$ & $16.0(2)$ & 12.8 & 24.7 & \multirow{5}{*}{$\begin{array}{c}0.026_{-0.016}^{+0.041} \\
0.56(5)\end{array}$} & \multirow{5}{*}{$\begin{array}{c}0.008 \\
0.48\end{array}$} \\
\hline & $2_{2}^{+} \rightarrow 0_{1}^{+}$ & $0.33(11)$ & 0.01 & 0.01 & & \\
\hline & $2_{3}^{+} \rightarrow 0_{1}^{+}$ & $2.2(2)$ & 1.54 & 1.66 & & \\
\hline & $2_{2}^{+} \rightarrow 2_{1}^{+}$ & $60_{-30}^{+20}$ & 17.4 & & & \\
\hline & $2_{3}^{+} \rightarrow 2_{1}^{+}$ & $4.9_{-2.3}^{+3.0}$ & 0.3 & & & \\
\hline \multirow{5}{*}{${ }^{92} \mathrm{Zr}$} & $2_{1}^{+} \rightarrow 0_{1}^{+}$ & $6.5(5)$ & 5.9 & 14.1 & \multirow{5}{*}{$\begin{array}{l}0.37(4) \\
<0.024\end{array}$} & \multirow{5}{*}{$\begin{array}{l}0.52 \\
0.02\end{array}$} \\
\hline & $2_{2}^{+} \rightarrow 0_{1}^{+}$ & $3.5(4)$ & 2.7 & 3.7 & & \\
\hline & $2_{3}^{+} \rightarrow 0_{1}^{+}$ & $<0.005$ & 0.1 & 0.1 & & \\
\hline & $2_{2}^{+} \rightarrow 2_{1}^{+}$ & $0.4_{-0.3}^{+0.5}$ & 0.2 & & & \\
\hline & $2_{3}^{+} \rightarrow 2_{1}^{+}$ & $<16$ & 6.4 & & & \\
\hline \multirow{5}{*}{${ }^{94} \mathrm{Zr}$} & $2_{1}^{+} \rightarrow 0_{1}^{+}$ & $4.9(11)$ & $\left.4.9^{*}\right)$ & $\left.15.0^{*}\right)$ & \multirow{5}{*}{$0.085_{-7}^{+6}$} & \multirow{5}{*}{$\left.0.40^{*}\right)$} \\
\hline & $2_{2}^{+} \rightarrow 0_{1}^{+}$ & $3.9(3)$ & $\left.3.4^{*}\right)$ & $\left.6.0^{*}\right)$ & & \\
\hline & $2_{2}^{+} \rightarrow 2_{1}^{+}$ & $0.061_{-0.06}^{+0.13}$ & $0.005^{*}$ & & & \\
\hline & $2_{3}^{+} \rightarrow 0_{1}^{+}$ & $0.019_{-0.012}^{+0.011}$ & $\left.0.005^{*}\right)$ & & & \\
\hline & $2_{3}^{+} \rightarrow 2_{1}^{+}$ & $60_{-30}^{+24}$ & $\left.49.1^{*}\right)$ & & & \\
\hline
\end{tabular}

") QRPA values

Table 8.3: Comparison of the calculated and measured transition strengths of quadrupole states. QPM $\mathrm{p}_{\mathrm{p}}$ denotes the electric transition strengths calculated in the QPM which are caused by the proton part of the wave function. Analogously one can calculate a $B(E 2)$-values for neutrons $\left(\mathrm{QPM}_{\mathrm{n}}\right)$. Experimental data is taken from Refs. [Fra03, Fra05, Elh08, Elh13a, Elh13b].

transition of the second $2^{+}$state to the $2_{1}^{+}$state is small and amounts to $B\left(E 2: 2_{1}^{+} \rightarrow 0_{1}^{+}\right)=$ $0.061_{-0.06}^{+0.13}$ W.u. i.e. it is in strong disagreement to the large value which would be obtained with the wave functions given in Tab. 8.2. Even a slight enhancement of the separation of the two unperturbed energies by a few hundred keV would drastically reduce the mixing and solve the discrepancy. It might be possible to achieve this additional separation by considering the quadrupole particle-particle interaction which is not considered in the calculations in this thesis. Due to this problem the interaction between different phonons is neglected in ${ }^{94} \mathrm{Zr}$ for the $2^{+}$states, i.e. in Tabs. 8.3 and 8.4 as well as in Figs. 8.4 and 8.5 the experimental data is compared to the QRPA-results. As mention in Sect. 5.3.1 it was necessary to lower the energy of the $\left(2 d_{5 / 2} \otimes 2 d_{5 / 2}\right)_{n}$-2qp-state artificially by $200 \mathrm{keV}$ in order the achieve a reasonable description of the $B(E 2)$-values in ${ }^{94} \mathrm{Zr}$. Without this change the QPM predicts the $B(E 2)$-value of the mixed-symmetry state a factor of four too low. This highlights that mixed-symmetry states are sensitive probes of the underlying shell structures. Therefore they are an important class of states and need to be studied.

The main signature of a one-phonon quadrupole mixed-symmetry state is an enhanced $B(M 1)$-value of the order of $\sim 1 \mu_{N}^{2}$ to the $2_{1}^{+}$state. As displayed in Tab. 8.3 this holds for the $2_{2}^{+}$states of ${ }^{92,94} \mathrm{Zr}$ and for the $2_{3}^{+}$state of ${ }^{94} \mathrm{Mo}$. Hence they were identified in the literature [Pie99, Fra03, Fra05, Elh08] as the states which carry the main fragment of the mixedsymmetry state. This view is supported by the QPM calculations. Particularly the QPM is able the reproduce the enhancement of the $B(M 1)$-values, although the experimental value is overes- 


\begin{tabular}{c|c|c|c}
\hline \hline${ }^{\mathrm{A}} X$ & $g\left(J^{\pi}\right)\left(\mu_{n}\right)$ & Exp & QPM \\
\hline${ }^{94} \mathrm{Mo}$ & $g\left(2_{1}^{+}\right)$ & $0.275(75)$ & 0.44 \\
\hline${ }^{92} \mathrm{Zr}$ & $g\left(2_{1}^{+}\right)$ & $-0.18(1)$ & -0.09 \\
& $g\left(2_{2}^{+}\right)$ & $0.76(50)$ & 0.73 \\
\hline${ }^{94} \mathrm{Zr}$ & $g\left(2_{1}^{+}\right)$ & $-0.32(2)$ & $\left.-0.13^{*}\right)$ \\
& $g\left(2_{2}^{+}\right)$ & $+0.88(27)$ & $\left.0.38^{*}\right)$ \\
\hline \hline
\end{tabular}

*) QRPA values

Table 8.4: Comparison of the calculated and measured g-factors of quadrupole states taken from Refs. [Wer08, Man01].

timated by a factor of five in ${ }^{94} \mathrm{Zr}$. The overestimation is partly due to the negligence of phonon mixing.

The mechanism for the strong M1-transition in a microscopic model like the QPM is the following. The proton and neutron g-factor have different signs as well as the main proton and neutron 2qp-states in the wave function of the mixed-symmetry state. Therefore the two M1-matrix elements of the main proton and neutron 2qp-states add up coherently. In addition it is possible that other $2^{+}$states do not have the right 2qp-composition to connect via the $M 1$ transition operator with the 2 qp-states of the $2_{1}^{+}$state. However, a pure $\left(1 g_{9 / 2} \otimes 1 g_{9 / 2}\right)_{p}$ would also have a large $M 1$-transition to the $2_{1}^{+}$state. A $M 1$-transition alone is not sufficient to identify a mixed-symmetry state unambiguously. In Sect. 8.3 a new signature is introduced which proves the mixed-symmetric character of the corresponding states in ${ }^{92,94} \mathrm{Zr}$ and ${ }^{94} \mathrm{Mo}$ independently of electromagnetic transition strengths.

In addition to energies and electromagnetic transition strengths g-factors are useful observables to test the QPM predictions. They provide information on the proton and neutron content of the wave functions. The $g$-factors of all three $2_{1}^{+}$states and the mixed-symmetry states of ${ }^{92,94} \mathrm{Zr}$ are shown in Tab. 8.4. For the $2_{3}^{+}$state in ${ }^{94}$ Mo no $g$-factor is known. The QPM results for the $g$-factors are in agreement with data. Especially the signs of the g-factors are correctly described. This gives additional support for the QPM wave functions in Tab. 8.1 and the simple two-state model introduced at the beginning of this section. Both models predict a neutron dominance of the $2_{1}^{+}$state in ${ }^{92,94} \mathrm{Zr}$ due to the large energy difference of the lowest proton and neutron 2qp-states. The negative signs of the $g$-factors confirm this prediction. Due to the reduced energy difference in ${ }^{94} \mathrm{Mo}$ a strong neutron dominance is not expected for the nucleus. Again the sign of the $g$-factor of the $2_{1}^{+}$state in ${ }^{94}$ Mo supports this assumption.

The $B(M 1)$-values in Tab. 8.3 and $g$-factors in Tab. 8.4 are sensitive to the main components of the neutron and proton parts of the wave functions. On the other hand absolute $E 2$-transition matrix elements to the ground state are mainly determined by high-lying 2qp-states which have large transition matrix elements but contribute with small amplitudes to the wave functions. This important point is discussed in detail in Sect 8.2. The correct description of $B(E 2)$-values proves that the QPM is capable of accounting for the collectivity of the proton components. The absolute proton scattering cross sections measured in this thesis allow to test if the same statement holds for the neutron part of the QPM wave functions.

Figure 8.4 compares the theoretical cross sections (dashed blue lines) with the experimental data for the transitions to low-lying $2^{+}$states. It was not possible to measure the cross section of the $2_{3}^{+}$state in ${ }^{94} \mathrm{Zr}$ which is expected to be a member of the two-phonon triplet due to its 
large $B(E 2)$-value to the $2_{1}^{+}$state. The calculations are based on the QPM wave functions and the effective nucleon-nucleus interaction of Sect. 5.1.2. The description of the cross sections of the $2_{1}^{+}$and $2_{\mathrm{ms}}^{+}$states is excellent in terms of magnitudes as well as of shapes. Proton scattering at $200 \mathrm{MeV}$ is sensitive to the proton and neutron transition matrix elements. Since the $B(E 2)$ values of the QPM are in agreement with the experimental ones the correct description of the proton scattering cross sections indicates that the neutron transition matrix elements of the QPM are reasonable.

The momentum transfers of the proton scattering cross sections in Fig. 8.4 are closely related to the transition radii [Wam10], i.e. they provide information on the radial structure of the excitation in contrast to transition matrix elements. A comparison of the cross sections of the $2_{1}^{+}$and $2_{\mathrm{ms}}^{+}$states reveals that both have very similar shapes but that the features of the cross sections of the $2_{\mathrm{ms}}^{+}$states are shifted to higher scattering angles (momentum transfers) with respect to the cross sections of the $2_{1}^{+}$states (see Fig. 8.17). These differences indicate that the transition radius of the $2_{\mathrm{ms}}^{+}$state is smaller than the transition radius of the $2_{1}^{+}$state. This is the case for all three investigated nuclei. In general the proton and neutron transition densities have different transition radii. Since proton scattering is sensitive to protons and neutrons the observable which is tested here is an 'isoscalar' transition radius (matter transition radius) and depends on the properties of both densities. However one can assume that the cross sections shown in Fig. 8.4 are more sensitive to the neutron transition densities for the following reasons. First of all, due to the properties of the effective nucleon-nucleus interaction, protons with an energy of $200 \mathrm{MeV}$ interact slightly stronger with neutrons than with protons [Lov81, Lov85]. Secondly, in the QPM the transition densities of the $2^{+}$states investigated in this thesis exhibit a small neutron 'skin'. Since proton scattering at the moment transfers used in this thesis probe mainly the surface of the nucleus one can expect that they mainly interact with neutrons.

In Sect. 8.3 it is shown that the shift of the features of the cross sections of the $2_{1}^{+}$and $2_{\mathrm{ms}}^{+}$ states is directly related to the mixed-symmetry character of the $2_{\mathrm{ms}}^{+}$state. The QPM is able to describe this shift in ${ }^{92,94} \mathrm{Zr}$ but underestimates its strength in ${ }^{94} \mathrm{Mo}$. In general the QPM is able to describe the transition radii of all investigated quadrupole states except the one of the $2_{\mathrm{ms}}^{+}$ state of ${ }^{94} \mathrm{Mo}$.

The DWBA-calculations fail to describe the cross section of the $2_{2}^{+}$and $2_{3}^{+}$stats of ${ }^{94}$ Mo and ${ }^{92} \mathrm{Zr}$ respectively. As discussed in Sect. 5.1 the calculations consider only direct excitations from the ground state and neglect coupled channel effects. If both states contain large two-phonon components $\left[2_{1}^{+} \otimes 2_{1}^{+}\right]_{[R P A]}$ the coupling to the $2_{1}^{+}$state is supposed to play a crucial role in the description of the cross sections. Unfortunately it is not possible to perform coupled channels calculations with the computer code DWBA07 [Ray07a]. In Refs. [Bur07, Wal10] some attempts were made to describe the angular distribution of the cross sections with the computer code CHUCK3 [Kun] which is able to include multi-step excitations. However the achieved description was not very good and some inconsistencies were found in the analysis. A more detailed theoretical analysis is required to confirm the assumption that the unusual cross sections of both states - which is indeed a strong hint for a dominant two-phonon component [Leo98] can be explained with the inclusion of multi-step processes.

Additional information on the proton transition densities can be obtained from electron scattering data. Electron scattering at low momentum transfers mainly probes the $B(E 2)$-values and the proton transition radii. The electron scattering form factor calculated in the framework of the QPM are compared to the data of Refs. [Bur07, Sch13] in Fig. 8.5. In ${ }^{92} \mathrm{Zr}$ an acceptable description of the experimental form factor is achieved indicating that the proton transition radii 

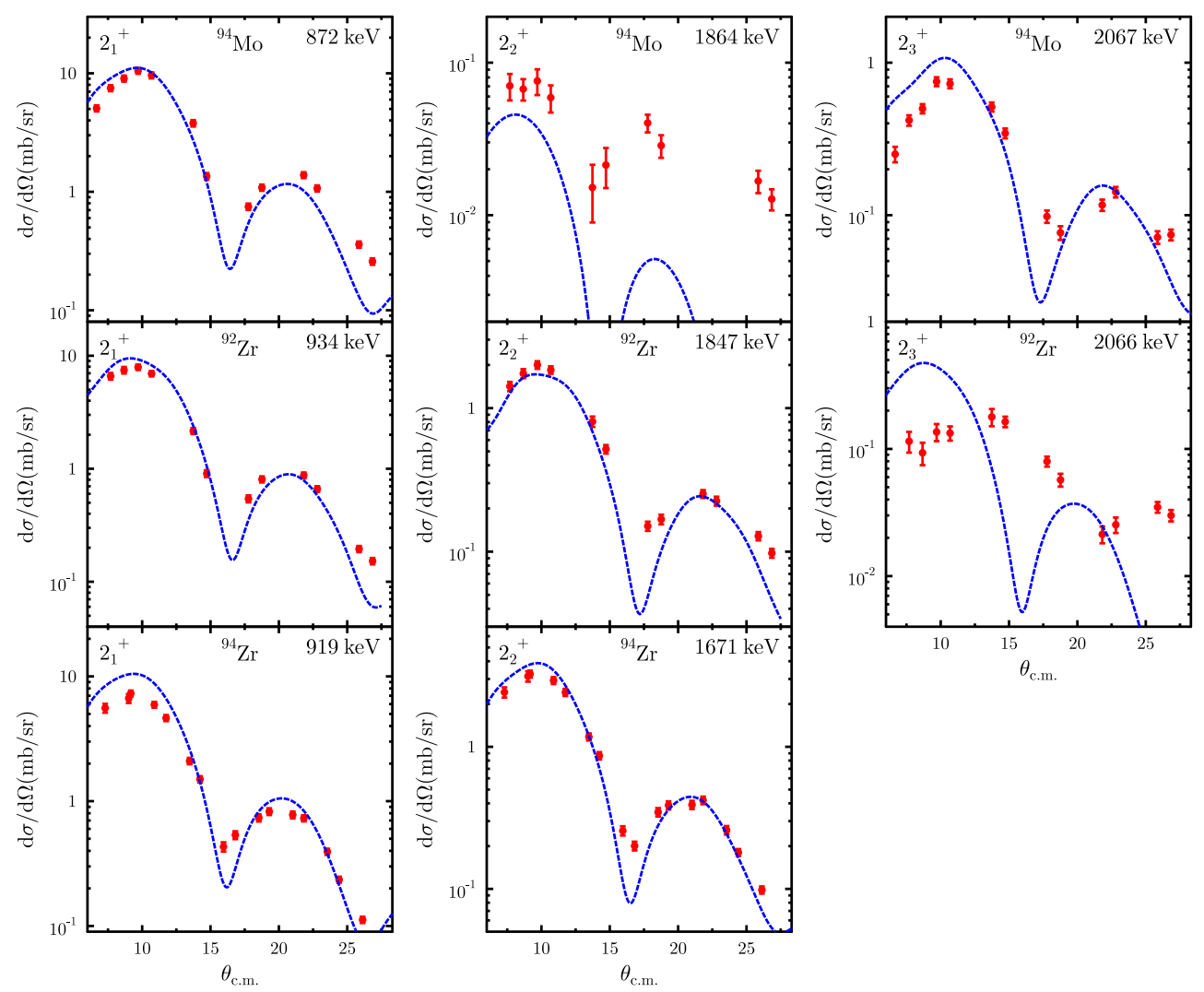

Figure 8.4: Comparison of the calculated and measured proton scattering cross sections of quadrupole states. The $2_{2}^{+}$state in ${ }^{94} \mathrm{Mo}$ and the $2_{3}^{+}$state in ${ }^{92} \mathrm{Zr}$ are expected to be two-phonon states. They are compared to the QPM calculations including only one-step excitations (dashed blue line). Note that the calculations for ${ }^{94} \mathrm{Zr}$ are on the QRPA level. For more details see text.

and $B(E 2)$-values of the QPM are reasonable. On the other hand in ${ }^{94}$ Mo the theoretical form factor is systematically shifted to higher momentum transfers with respect to the experimental one. This points to an underestimation of the proton transition radii. In order to fix the problem it was tried to change slightly the parameters of the Wood-Saxon potential but this did not improve the quality of the theoretical description.

\subsubsection{Hexadecapole states}

Table 8.5 shows the structure of the first and second hexadecapole QRPA-phonons of ${ }^{92,94} \mathrm{Zr}$ and ${ }^{94}$ Mo. The same 2-qp states are important, as for the quadrupole states, namely the $\left(2 d_{5 / 2} \otimes\right.$ $\left.2 d_{5 / 2}\right)_{n}$ - and $\left(1 g_{9 / 2} \otimes 1 g_{9 / 2}\right)_{p}$-configurations. Again one can understand the structure of the phonons in the simple two-state model introduced for the $2^{+}$states. Two states are formed in this model: one where both 2qp-states are connected with a plus sign and one where both are connected with a minus sign. The same structure can be found in the QRPA calculations.

Two important differences are apparent in comparison to the results of the quadrupole phonons shown in Tab. 8.1. First, the mixing between both 2qp-states is much weaker. The $\left[4_{1}^{+}\right]_{[R P A]}$-phonons of all three nuclei have an overwhelming neutron dominance and are basically pure $\left(2 d_{5 / 2} \otimes 2 d_{5 / 2}\right)_{n}$-2qp-states. This can be explained with the properties of the hex- 

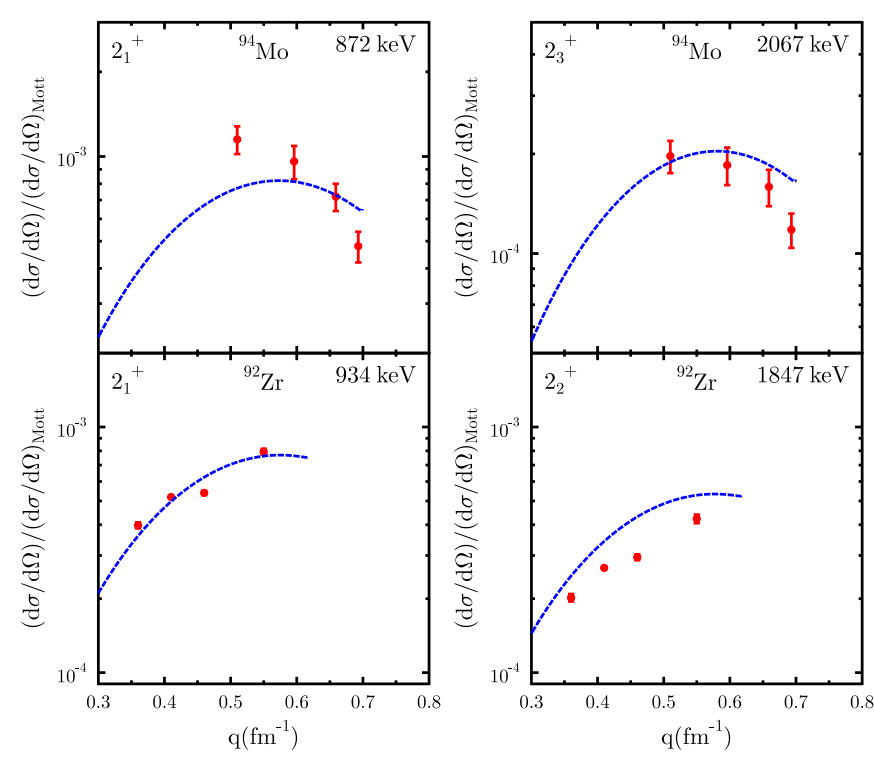

Figure 8.5: Comparison of the calculated and measured electron scattering cross sections of the quadrupole states. For the nucleus ${ }^{94} \mathrm{Zr}$ no data was available at the time this thesis was written. Note that the beam energy was constant for each nucleus. (The data is taken from Refs. [Bur07, Wal11].)

\begin{tabular}{c|c|ccc|ccc}
\hline \hline \multirow{3}{*}{${ }^{\mathrm{A}} X$} & \multicolumn{3}{|c|}{$\left(2 d_{5 / 2} \otimes 2 d_{5 / 2}\right)_{n}$} & \multicolumn{3}{|c}{$\left(1 g_{9 / 2} \otimes 1 g_{9 / 2}\right)_{p}$} \\
& QRPA-phonons & $\psi$ & $\phi$ & $\%$ & $\psi$ & $\phi$ & $\%$ \\
\hline${ }^{94} \mathrm{Mo}$ & {$\left[4_{1}^{+}\right]_{[R P A]}$} & 1.34 & 0.04 & 90.30 & 0.41 & 0.08 & 8.17 \\
& {$\left[4_{2}^{+}\right]_{[R P A]}$} & -0.44 & 0.05 & 9.43 & 1.30 & 0.06 & 84.95 \\
\hline${ }^{92} \mathrm{Zr}$ & {$\left[4_{1}^{+}\right]_{[R P A]}$} & 1.39 & 0.04 & 96.22 & 0.22 & 0.06 & 2.21 \\
& {$\left[4_{2}^{+}\right]_{[R P A]}$} & -0.26 & 0.05 & 3.32 & 1.29 & 0.06 & 83.47 \\
\hline${ }^{94} \mathrm{Zr}$ & {$\left[4_{1}^{+}\right]_{[R P A}$} & 1.40 & 0.04 & 98.14 & 0.15 & 0.05 & 0.93 \\
& {$\left[4_{2}^{+}\right]_{[R P A}$} & -0.18 & 0.05 & 1.51 & 1.00 & 0.06 & 50.19 \\
\hline \hline
\end{tabular}

Table 8.5: Same as Tab. 8.1 but for hexadecapole QRPA-phonons.

adecapole proton-neutron residual interaction which is significantly weaker than the protonneutron quadrupole residual interaction (note that the energy difference between the unperturbed 2qp-states is the same for quadrupole and hexadecapole 2qp-states). Like for the $2^{+}$ states both 2qp-states are more admixed in case of ${ }^{94} \mathrm{Mo}$ than for ${ }^{92,94} \mathrm{Zr}$ due to the smaller energy difference between the $\left(2 d_{5 / 2} \otimes 2 d_{5 / 2}\right)_{n}$ - and $\left(1 g_{9 / 2} \otimes 1 g_{9 / 2}\right)_{p}$-configurations.

Secondly other 2qp-states than the $\left(2 d_{5 / 2} \otimes 2 d_{5 / 2}\right)_{n}$ - and $\left(1 g_{9 / 2} \otimes 1 g_{9 / 2}\right)_{p}$-configurations play a less important role. Their contribution to the norm of the wave functions is for each phonon less than $15 \%$ (except for the $\left[4_{2}^{+}\right]_{[R P A]}$-phonon of ${ }^{94} \mathrm{Zr}$ ), i.e. the simple two-state model is a good approximation of the QRPA wave functions. One reason is again the weak hexadecapole residual interaction reducing the mixing of higher-lying 2 qp-states with $\left(2 d_{5 / 2} \otimes 2 d_{5 / 2}\right)_{n}$ - and $\left(1 g_{9 / 2} \otimes\right.$ $\left.1 g_{9 / 2}\right)_{p}$-2qp-states. Additionally some 2qp-states - which are relatively close in energy to the two imporant 2qp-states - cannot couple to $J=4$. One example is the $\left(2 d_{5 / 2} \otimes 3 s_{1 / 2}\right)_{n}$-2qp-state (see Fig. 5.4). In the sense of the definition of a mixed-symmetry state given in Sect. 5.3.3 the QPM predicts a hexadecapole mixed-symmetric phonon. The formation mechanism is similar 
to the quadrupole states although the mixing of the two important 2qp-states is dramatically weaker.

\begin{tabular}{c|c|cc|c}
\hline \hline${ }^{\mathrm{A}} \mathrm{X}$ & state & \multicolumn{2}{|c|}{$\mathrm{E}(\mathrm{keV})$} & structure \\
& & $\mathrm{Exp}$ & $\mathrm{QPM}$ & \\
\hline \multirow{2}{*}{${ }^{94} \mathrm{Mo}$} & $4_{1}^{+}$ & 1574 & 1205 & $43 \%\left[4_{1}^{+}\right]_{[R P A]}+44 \%\left[2_{1}^{+} \otimes 2_{1}^{+}\right]_{[R P A]}$ \\
& $4_{2}^{+}$ & 2295 & 1976 & $-28 \%\left[4_{1}^{+}\right]_{[R P A]}+10 \%\left[4_{2}^{+}\right]_{[R P A]}-10 \%\left[2_{1}^{+} \otimes 2_{1}^{+}\right]_{[R P A]}$ \\
& $4_{4}^{+}$ & 2768 & 2699 & $43 \%\left[4_{2}^{+}\right]_{[R P A]}-24 \%\left[2_{1}^{+} \otimes 2_{1}^{+}\right]_{[R P A]}$ \\
\hline \multirow{2}{*}{$\mathrm{P2} \mathrm{Zr}$} & $4_{1}^{+}$ & 1496 & 1495 & $56 \%\left[4_{1}^{+}\right]_{[R P A]}+29 \%\left[2_{1}^{+} \otimes 2_{1}^{+}\right]_{[R P A]}$ \\
& $4_{2}^{+}$ & 2398 & 1951 & $-36 \%\left[4_{1}^{+}\right]_{[R P A]}+6 \%\left[4_{2}^{+}\right]_{[R P A]}+24 \%\left[2_{1}^{+} \otimes 2_{1}^{+}\right]_{[R P A]}$ \\
& $4_{3}^{+}$ & 2865 & 2643 & $37 \%\left[4_{2}^{+}\right]_{[R P A]}+34 \%\left[2_{1}^{+} \otimes 4_{1}^{+}\right]_{[R P A]}$ \\
\hline${ }^{94} \mathrm{Zr}$ & $4_{1}^{+}$ & 1470 & 1404 & $76 \%\left[4_{1}^{+}\right]_{[R P A]}+16 \%\left[2_{1}^{+} \otimes 2_{1}^{+}\right]_{[R P A]}$ \\
& $4_{2}^{+}$ & 2330 & 2584 & $48 \%\left[4_{2}^{+}\right]_{[R P A]}+17 \%\left[2_{1}^{+} \otimes 2_{2}^{+}\right]_{[R P A]}$ \\
\hline \hline
\end{tabular}

Table 8.6: Same as Tab. 8.2 but for hexadecapole QPM states.

Tab. 8.6 displays the final QPM wave functions of the lowest $4^{+}$states of ${ }^{92,94} \mathrm{Zr}$ and ${ }^{94} \mathrm{Mo}$. The leading components are the $\left.\left[4_{1}^{+}\right]_{[R P A}\right]^{-},\left[4_{2}^{+}\right]_{[R P A]^{-}}$and $\left[2_{1}^{+} \otimes 2_{1}^{+}\right]_{[R P A]}$-phonons. This is a similar situation like for the quadrupole states where the $\left[2_{1}^{+}\right]_{[R P A]^{-}},\left[2_{2}^{+}\right]_{[R P A]^{-}}$and $\left[2_{1}^{+} \otimes 2_{1}^{+}\right]_{[R P A]^{-}}$ phonons determined the properties of the low-lying $2^{+}$states. In case of the $4^{+}$states, however, the three important phonons are much closer in energy leading to a strong mutual mixing. For example the $\left[4_{1}^{+}\right]_{[R P A]^{-}}$and $\left[2_{1}^{+} \otimes 2_{1}^{+}\right]_{[R P A]}$-phonons contribute with sizable amplitudes to the $4_{1}^{+}$state of ${ }^{94} \mathrm{Mo}$. This is in sharp contrast to the wave functions of the $2^{+}$states where each of the three important phonons formed basically also one final QPM state, i.e. the mixing between them is weak due to the larger energy difference of the unperturbed phonon energies. The more complicated situation of the $4^{+}$states makes it difficult to identify the fragments of the mixed-symmetric QRPA-phonons unambiguously. This will be discussed in the following.

The energies of the $4^{+}$states calculated in the framework of the QPM are compared to the experimental ones in Tab. 8.6. The QPM is not able to reproduce the experimental energies with a satisfactory accuracy. Deviations can be found for all three nuclei of several hundred keV. Especially in ${ }^{94} \mathrm{Zr}$ the calculations fail because the QPM predicts the $4_{2}^{+}$state at an energy of $\sim 1500 \mathrm{keV}$ (not shown in Tab. 8.6), i.e. very close to the $4_{1}^{+}$state which is in strong disagreement with experiment. Hence the properties of the third $4^{+}$state of the QPM are compared to the experimental $4_{2}^{+}$state in Tab. 8.7 and Fig. 8.6. Due to these problems ${ }^{94} \mathrm{Zr}$ is excluded from the following discussion. Since the unperturbed energies of the $\left.\left[4_{1}^{+}\right]_{[R P A]^{-}},\left[4_{2}^{+}\right]_{[R P A}\right]^{-}$and $\left[2_{1}^{+} \otimes 2_{1}^{+}\right]_{[R P A]}$-phonons are close to each other the final QPM wave functions are highly sensitive to the details of the calculations. Already small changes of the strength of the residual interaction change the phonon structure of the final QPM wave functions and the corresponding energies and transition strengths significantly. Given this it is not a surprise that the QPM has problems to describe the properties of the $4^{+}$states.

The experimental and theoretical transition strengths are shown in Tab. 8.5. The $4_{1}^{+}$and $4_{2}^{+}$ states of ${ }^{94} \mathrm{Mo}$ and ${ }^{92} \mathrm{Zr}$ are connected with large $B(M 1)$-values of $1.23(30) \mu_{N}^{2}$ and $0.26(3) \mu_{N}^{2}$ respectively. Also the $B\left(M 1 ; 4_{3}^{+} \rightarrow 4_{1}^{+}\right)=0.23(6) \mu_{N}^{2}$ value in ${ }^{94}$ Mo is sizable. The main experimental signature for a quadrupole mixed-symmetry state is an enhanced $B(M 1)$-value to the $2_{1}^{+}$ state. Does this signature also hold for the $4^{+}$states, i.e. carry the $4_{2}^{+}$and $4_{3}^{+}$states in ${ }^{94} \mathrm{Mo}$ and the $4_{2}^{+}$state in ${ }^{92} \mathrm{Zr}$ large fractions of the mixed-symmetry state? 


\begin{tabular}{|c|c|c|c|c|c|c|}
\hline \multirow[b]{2}{*}{${ }^{\mathrm{A}} X$} & \multirow[b]{2}{*}{$J_{i} \rightarrow J_{f}$} & \multicolumn{3}{|c|}{$B(E J)($ W.u.) } & \multicolumn{2}{|c|}{$B(M 1)\left(\mu_{N}^{2}\right)$} \\
\hline & & Exp & $\mathrm{QPM}_{\mathrm{p}}$ & $\mathrm{QPM}_{\mathrm{n}}$ & exp & QPM \\
\hline \multirow{8}{*}{${ }^{94} \mathrm{Mo}$} & $4_{1}^{+} \rightarrow 0_{1}^{+}$ & & 2.1 & 16.2 & & \\
\hline & $4_{2}^{+} \rightarrow 0_{1}^{+}$ & & 0.81 & 1.1 & & \\
\hline & $4_{4}^{+} \rightarrow 0_{1}^{+}$ & & 1.93 & 5.8 & & \\
\hline & $4_{2}^{+} \rightarrow 4_{1}^{+}$ & $1.2(33)$ & 1.7 & & $1.23(30)$ & $0.30(0.12)^{*}$ \\
\hline & $4_{3}^{+} \rightarrow 4_{1}^{+}$ & & 1.6 & & $0.23(6)$ & $\left.0.82(0.62)^{*}\right)$ \\
\hline & $4_{4}^{+} \rightarrow 4_{1}^{+}$ & $36_{-4}^{+5}$ & 0.3 & & $0.090(11)$ & $\left.0.23(0.63)^{*}\right)$ \\
\hline & $4_{1}^{+} \rightarrow 2_{1}^{+}$ & $26.0_{-3.2}^{+4.2}$ & 12.6 & & & \\
\hline & $4_{2}^{+} \rightarrow 2_{1}^{+}$ & $5.9_{-0.8}^{+1.0}$ & 2.7 & & & \\
\hline \multirow{8}{*}{${ }^{92} \mathrm{Zr}$} & $4_{1}^{+} \rightarrow 0_{1}^{+}$ & & 1.4 & 5.0 & & \\
\hline & $4_{2}^{+} \rightarrow 0_{1}^{+}$ & & 0.15 & 0.53 & & \\
\hline & $4_{3}^{+} \rightarrow 0_{1}^{+}$ & & 1.4 & 1.9 & & \\
\hline & $4_{2}^{+} \rightarrow 4_{1}^{+}$ & $2.3(11)$ & 0.2 & & $0.26(3)$ & 0.32 \\
\hline & $4_{1}^{+} \rightarrow 2_{1}^{+}$ & $4.05(11)$ & 3.8 & & & \\
\hline & $4_{2}^{+} \rightarrow 2_{1}^{+}$ & $6.1(8)$ & 3.1 & & & \\
\hline & $4_{3}^{+} \rightarrow 2_{1}^{+}$ & & 0.1 & & & \\
\hline & $4_{4}^{+} \rightarrow 2_{1}^{+}$ & & 2.0 & & & \\
\hline \multirow{5}{*}{${ }^{94} \mathrm{Zr}$} & $4_{1}^{+} \rightarrow 0_{1}^{+}$ & & 1.1 & 8.4 & & \multirow{5}{*}{0.2} \\
\hline & $4_{2}^{+} \rightarrow 0_{1}^{+}$ & & 1.9 & 6.0 & & \\
\hline & $4_{2}^{+} \rightarrow 4_{1}^{+}$ & & & & & \\
\hline & $4_{1}^{+} \rightarrow 2_{1}^{+}$ & $0.880(23)$ & 0.9 & & & \\
\hline & $4_{2}^{+} \rightarrow 2_{1}^{+}$ & $13_{-7}^{+4}$ & 3.3 & & & \\
\hline
\end{tabular}

") In brackets are M1-transitions between one-phonon $4^{+}$configurations only.

Table 8.7: Same as Tab. 8.3 but for hexadecapole states. Experimental data is taken from Refs. [Fra03, Fra05, Elh08, Elh13a, Elh13b].

To answer this question it is useful to have a look at the M1-transitions between pure phonon configurations. For ${ }^{94}$ Mo the following $B(M 1)$-values are obtained: $B\left(M 1 ;\left[4_{1}^{+}\right]_{[R P A]} \rightarrow\right.$ $\left.\left[4_{1}^{+}\right]_{[R P A]}\right)=0.35 \mu_{N}^{2}, B\left(M 1 ;\left[4_{2}^{+}\right]_{[R P A]} \rightarrow\left[4_{1}^{+}\right]_{[R P A]}\right)=1.2 \mu_{N}^{2}$ and $B\left(M 1 ;\left[2_{1}^{+} \otimes 2_{1}^{+}\right]_{[R P A]} \rightarrow\left[2_{1}^{+} \otimes\right.\right.$ $\left.\left.2_{1}^{+}\right]_{[R P A]}\right)=0.9 \mu_{N}^{2}$. The $B\left(M 1:\left[4_{2}^{+}\right]_{[R P A]} \rightarrow\left[4_{1}^{+}\right]_{[R P A]}\right)$-value is indeed large but not strongly enhance in comparison to the $B\left(M 1 ;\left[4_{1}^{+}\right]_{[R P A]} \rightarrow\left[4_{1}^{+}\right]_{[R P A]}\right)$ - and $B\left(M 1 ;\left[2_{1}^{+} \otimes 2_{1}^{+}\right]_{[R P A]} \rightarrow\right.$ $\left.\left[2_{1}^{+} \otimes 2_{1}^{+}\right]_{[R P A]}\right)$-values which have a similar size. The latter are the squares of the diagonal matrix elements of the $M 1$-transition operator and are closely related to $g$-factors. Therefore in case of strong phonon mixing - that is predicted by the QPM for the three nuclei considered - a large $M 1$-transition is not a reliable signature to identify hexadecapole mixed-symmetry states. For example the $\left[4_{1}^{+}\right]_{[R P A]^{-}}$and $\left[2_{1}^{+} \otimes 2_{1}^{+}\right]_{[R P A]}$-phonons contribute with sizable amplitudes to the $4_{1}^{+}$and $4_{2}^{+}$states of ${ }^{94} \mathrm{Mo}$. A significant part of the $\mathrm{B}\left(\mathrm{M} 1: 4_{2}^{+} \rightarrow 4_{1}^{+}\right)$-value of $0.3 \mu_{N}^{2}$ predicted by the QPM is due to contributions from diagonal M1-transitions i.e. $\left[4_{1}^{+}\right]_{[R P A]} \rightarrow\left[4_{1}^{+}\right]_{[R P A]}$ and $\left[2_{1}^{+} \otimes 2_{1}^{+}\right]_{[R P A]} \rightarrow\left[2_{1}^{+} \otimes 2_{1}^{+}\right]_{[R P A]}$.

There are indications in the experimental data supporting strong phonon mixing. The $4_{1}^{+}$state of ${ }^{94} \mathrm{Mo}$ has a large $B(E 2)$-value of $26.0_{-3.2}^{+4.2}$ W.u. to the $2_{1}^{+}$indicating a large $\left[2_{1}^{+} \otimes 2_{1}^{+}\right]_{[R P A]^{-}}$ component. On the other hand the proton scattering cross sections shown in Fig. 8.6 and the electron scattering form factor of Fig. 8.7 give evidence of large one-phonon components in the 
wave function. Both cross sections are large and the proton scattering cross section is described well by considering a one-step excitation mechanism only. Furthermore the $4_{2}^{+}$state has also a sizable $B(E 2)$-value to the $2_{1}^{+}$state of $5.9_{-0.8}^{+1.0} \mathrm{~W}$.u. which is most likely caused by a $\left[2_{1}^{+} \otimes 2_{1}^{+}\right]_{[R P A]^{-}}$ component in the wave function. Its proton scattering cross section has a very similar shape than the one of the $4_{1}^{+}$state. This is a hint that the same QRPA-phonon is important for both states. Similar observations can be made for the $4_{1}^{+}$and $4_{2}^{+}$states of ${ }^{92} \mathrm{Zr}$.

The identification of the main fragments of the hexadecapole mixed-symmetry state seems only to be possible in the framework of a nuclear structure model. One has to find a model which describes sufficiently well all important experimental observables and inspect the resulting wave function. An unambiguous experimental observable like for the $2^{+}$states seems not to be available. Unfortunately the QPM does not account so well for the properties of the $4^{+}$ states.
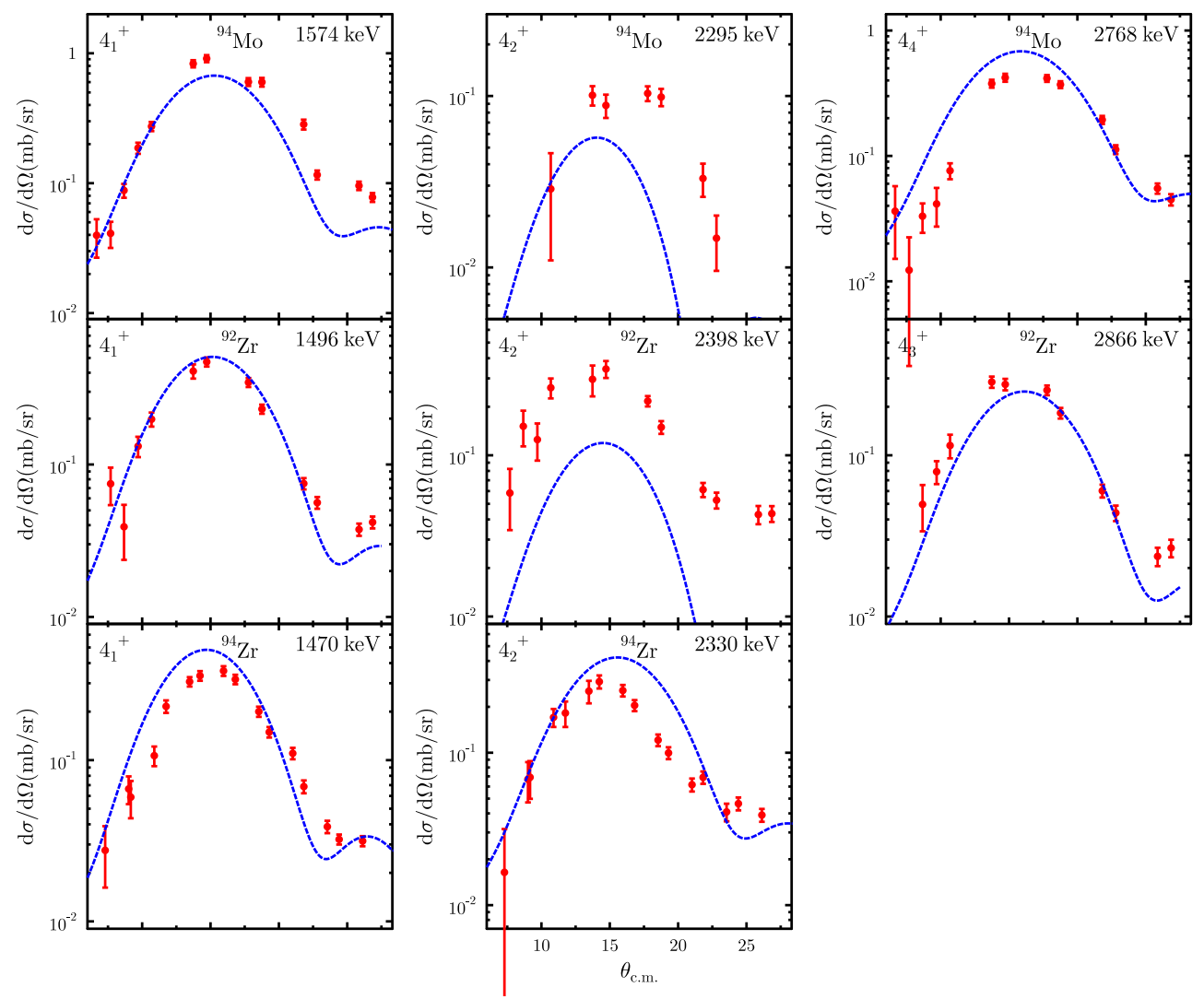

Figure 8.6: Same as Fig. 8.4 but for hexadecapole states.

The QPM has also problems to describe the experimental transition strengths in Tab. 8.7. The important $B\left(M 1 ; 4_{2}^{+} \rightarrow 4_{1}^{+}\right)$-value is underestimated by a factor of four in ${ }^{94} \mathrm{Mo}$. For each $B(M 1)$-value of ${ }^{94} \mathrm{Mo}$ in Tab. 8.7 also the part stemming from the one-phonon components only is given in brackets, i.e. more than $50 \%$ of the $B\left(M 1 ; 4_{2}^{+} \rightarrow 4_{1}^{+}\right)$-value is due to transitions between two-phonon components. Also the $B\left(E 2 ; 4_{1}^{+} \rightarrow 2_{1}^{+}\right)$- and $B\left(E 2 ; 4_{2}^{+} \rightarrow 2_{1}^{+}\right)$-values are both underestimated by more than a factor of two. This is a clear indication that the QPM amplitude of the $\left[2_{1}^{+} \otimes 2_{1}^{+}\right]_{[R P A]^{-}}$-component in both states is too small. An increase of this component would also improve the description of the $\mathrm{B}\left(\mathrm{M} 1: 4_{2}^{+} \rightarrow 4_{1}^{+}\right)$-value since the diagonal M1-matrix element of the $\left[2_{1}^{+} \otimes 2_{1}^{+}\right]_{[R P A]}$-phonon is large. On the other hand the descriptions of the proton and electron scattering cross sections of the $4_{1}^{+}$state of Figs. 8.6 and 8.7 - which 

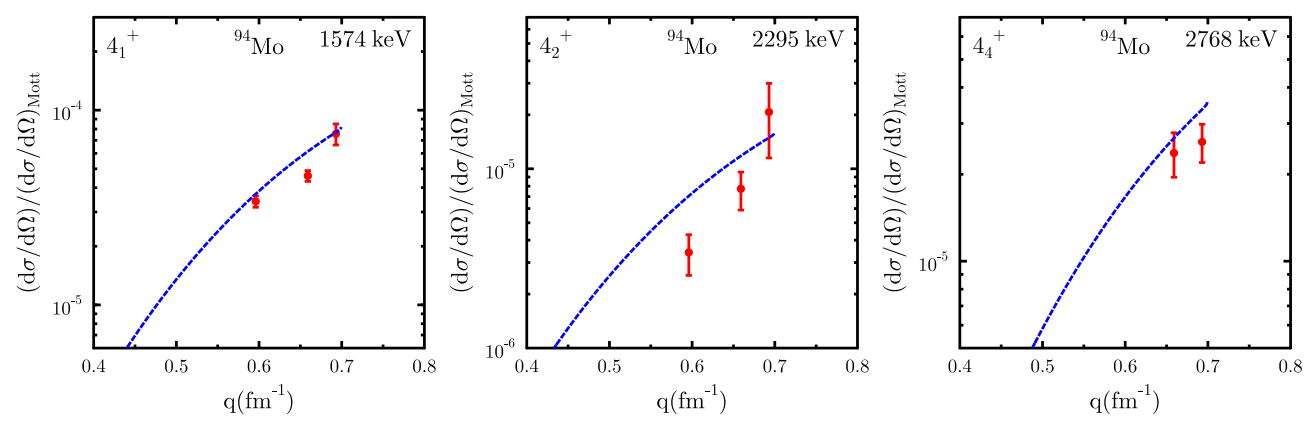

Figure 8.7: Same as Fig. 8.5 but for hexadecapole states.

are excellent - would become worse. A simple redistribution of the phonons does not seem to improve the overall description of the experimental obvervables. This points to problems with the internal structures of the hexadecapole phonons in ${ }^{94} \mathrm{Mo}$.

The theoretical proton scattering cross section of the $4_{2}^{+}$state in ${ }^{94} \mathrm{Mo}$ is very unusual which is in disagreement with the experimental data. In contrast to all other $4^{+}$its shape is shifted to smaller scattering angles. This is caused by destructive inference of the $\left[4_{1}^{+}\right]_{[R P A]^{-}}$and $\left[4_{2}^{+}\right]_{[R P A]^{-}}$ phonons which both contribute to the $4_{2}^{+}$state.

The QPM predicts the main fragment of the mixed-symmetric phonon $\left[4_{2}^{+}\right]_{[R P A]}$ in the $4_{4}^{+}$ state. The description of the electron and proton scattering cross sections is acceptable but the $B(M 1)$-value is significantly overestimated. This state has a very large E2-transition of $36_{-4}^{+5}$ W.u. to the $4_{1}^{+}$state. The QPM underestimates this transition by 2 orders of magnitude which clearly shows that the QPM results are not reliable for this state. Such a large B(E2)-value can be in parts explained with a large $\left[4_{1}^{+} \otimes 2_{1}^{+}\right]_{[R P A]}$-component contributing to the wave function.

The QPM is more successful in describing the properties of the $4^{+}$states of ${ }^{92} \mathrm{Zr}$. The $B\left(E 2 ; 4_{1}^{+} \rightarrow 2_{1}^{+}\right)$-value is in good agreement with experiment as well as the $M 1$-matrix element of the $4_{2}^{+} \rightarrow 4_{1}^{+}$transition. The $B\left(E 2 ; 4_{2}^{+} \rightarrow 2_{1}^{+}\right)$-value, however, is again a factor of two larger in experiment. Interestingly the main fragment of the $\left[2_{1}^{+} \otimes 2_{1}^{+}\right]_{[R P A]}$-phonon is in ${ }^{92} \mathrm{Zr}$ in the second $4^{+}$and not in the $4_{1}^{+}$state as in ${ }^{94} \mathrm{Mo}$. Although the proton scattering cross section of the $4_{1}^{+}$and $4_{3}^{+}$states are very well described in terms of shape and magnitude, the cross section of the $4_{2}^{+}$state is significantly underestimated.

\begin{tabular}{c|c|c|c}
\hline \hline${ }^{\mathrm{A}} X$ & $g\left(J^{\pi}\right)\left(\mu_{n}\right)$ & Exp & QPM \\
\hline${ }^{92} \mathrm{Zr}$ & $g\left(4_{1}^{+}\right)$ & $-0.5(1)$ & -0.55 \\
\hline \hline
\end{tabular}

Table 8.8: Same as Tab. 8.4 but for hexadecapole states.

Only the $g$-factor of the $4_{1}^{+}$state in ${ }^{92} \mathrm{Zr}$ is known for the $4^{+}$states. It is large and negative and well reproduced by the QPM indicating a dominant neutron component contributing to the wave function.

\subsubsection{Octupole and $5^{-}$states}

This section examines the properties of the $3^{-}$and $5^{-}$states of ${ }^{92,94} \mathrm{Zr}$ and ${ }^{94} \mathrm{Mo}$. The structure of the $3^{-}$states of ${ }^{94} \mathrm{Mo}$ was already discussed in the author's master thesis [Wal10]. Most of the statements made in this thesis are still valid and are repeated here. However, no comparison 
was made to experimental electron or proton scattering cross sections. This new information is discussed in the present thesis. Furthermore the analysis is extended to ${ }^{92,94} \mathrm{Zr}$.

\begin{tabular}{c|c|ccc|ccc}
\hline \hline \multirow{2}{*}{${ }^{\mathrm{A}} X$} & \multicolumn{3}{|c|}{$\left(2 d_{5 / 2} \otimes 1 h_{11 / 2}\right)_{n}$} & \multicolumn{3}{|c}{$\left(2 \mathrm{p}_{3 / 2} \otimes 1 g_{9 / 2}\right)_{p}$} \\
& QRPA-phonons & $\psi$ & $\phi$ & $\%$ & $\psi$ & $\phi$ & $\%$ \\
\hline${ }^{94} \mathrm{Mo}$ & {$\left[3_{1}^{-}\right]_{[R P A]}$} & 0.44 & 0.13 & 18.01 & 0.73 & 0.18 & 49.87 \\
& {$\left[3_{3}^{-}\right]_{[R P A]}$} & -0.84 & 0.00 & 71.72 & 0.47 & -0.03 & 21.89 \\
\hline${ }^{92} \mathrm{Zr}$ & {$\left[3_{1}^{-}\right]_{[R P A]}$} & 0.38 & 0.12 & 13.40 & 0.80 & 0.17 & 60.39 \\
& {$\left[3_{3}^{-}\right]_{[R P A]}$} & -0.89 & 0.00 & 78.35 & 0.41 & -0.04 & 16.56 \\
\hline${ }^{94} \mathrm{Zr}$ & {$\left[3_{1}^{-}\right]_{[R P A]}$} & 0.58 & 0.20 & 29.59 & 0.72 & 0.21 & 46.99 \\
& {$\left[3_{3}^{-}\right]_{[R P A]}$} & -0.79 & 0.01 & 62.26 & 0.54 & -0.03 & 29.27 \\
\hline \hline
\end{tabular}

Table 8.9: Same as Tab. 8.1 but for octupole QRPA-phonons.

Due to the negative parity different 2qp-states are important for the $3^{-}$and $5^{-}$states compared to $2^{+}$and $4^{+}$states. In order to form low-lying one-phonon symmetric and mixedsymmetric states proton and neutron 2qp-states have to be present at low energies. Therefore it is necessary that the unique parity single-particle states contribute to the two main components because cross-shell excitations would be too high in energy. In the investigated nuclei the unique parity single-particle states are $1 h_{11 / 2}$ for neutrons and $1 g_{9 / 2}$ for protons. Indeed both contribute to the two dominant 2-qp components $\left(2 d_{5 / 2} \otimes 1 h_{11 / 2}\right)_{n}$ and $\left(2 p_{3 / 2} \otimes 1 g_{9 / 2}\right)_{p}$ which determine the structure of the $\left[3_{1}^{-}\right]_{[R P A]^{-}}$and $\left[3_{3}^{-}\right]_{[R P A]}$-phonons (see Tab. 8.9). Like for the $2^{+}$ and $4^{+}$states the two main 2qp-components are in-phase for $\left[3_{1}^{-}\right]_{[R P A]}$-phonon and out-of-phase $\left[3_{3}^{-}\right]_{[R P A]}$-phonon, i.e. the QPM predicts an octupole mixed-symmetric phonon. The $\left[3_{2}^{-}\right]_{[R P A]^{-}}$ phonon is a pure 2qp-state and is not further considered here. Again the formation mechanism and the degree of mixing can be understood in the simple two-state model.

\begin{tabular}{c|c|cc|c}
\hline \hline${ }^{\mathrm{A}} X$ & state & \multicolumn{2}{|c|}{$E(\mathrm{keV})$} & structure \\
& & $\operatorname{Exp}$ & $\mathrm{QPM}$ & \\
\hline \multirow{3}{*}{${ }^{94} \mathrm{Mo}$} & $3_{1}^{-}$ & 2534 & 2429 & $86 \%\left[3_{1}^{-}\right]_{[R P A]}+10 \%\left[2_{1}^{+} \otimes 3_{1}^{-}\right]_{[R P A]}$ \\
& $3_{2}^{-}$ & 3012 & 3238 & $10 \%\left[3_{1}^{-}\right]_{[R P A]}+11 \%\left[3_{3}^{-}\right]_{[R P A]}-57 \%\left[2_{1}^{+} \otimes 3_{1}^{-}\right]_{[R P A]}$ \\
& $5_{1}^{-}$ & 2611 & 2422 & $92 \%\left[5_{1}^{-}\right]_{[R P A]}$ \\
\hline \multirow{3}{*}{$\mathrm{Zr} \mathrm{Zr}$} & $3_{1}^{-}$ & 2340 & 2342 & $88 \%\left[3_{1}^{-}\right]_{[R P A]}+11 \%\left[2_{1}^{+} \otimes 3_{1}^{-}\right]_{[R P A]}$ \\
& $3_{2}^{-}$ & 3446 & 3766 & $7 \%\left[3_{1}^{-}\right]_{[R P A]}-12 \%\left[3_{3}^{-}\right]_{[R P A]}-55 \%\left[2_{1}^{+} \otimes 3_{1}^{-}\right]_{[R P A]}$ \\
& $5_{1}^{-}$ & 2486 & 2432 & $94 \%\left[5_{1}^{-}\right]_{[R P A}$ \\
\hline${ }^{94} \mathrm{Zr}$ & $3_{1}^{-}$ & 2058 & 2030 & $94 \%\left[3_{1}^{-}\right]_{[R P A]}$ \\
& $3_{2}^{-}$ & 3232 & 2879 & $5 \%\left[3_{1}^{-}\right]_{[R P A]}+3 \%\left[3_{3}^{-}\right]_{[R P A]}-82 \%\left[2_{1}^{+} \otimes 3_{1}^{-}\right]_{[R P A]}$ \\
& $5_{1}^{-}$ & 2605 & 2269 & $92 \%\left[5_{1}^{-}\right]_{[R P A]}$ \\
\hline \hline
\end{tabular}

Table 8.10: Same as Tab. 8.2 but for octupole and 5- QPM states.

The final QPM states are shown in Tab. 8.10. The $3_{1}^{-}$states in ${ }^{92} \mathrm{Zr}$ and ${ }^{94} \mathrm{Mo}$ are dominated by the $\left[3_{1}^{-}\right]_{[R P A]}$-phonons but also the $\left[2_{1}^{+} \otimes 3_{1}^{-}\right]_{[R P A]}$-components contribute with sizable amplitudes. The $3_{2}^{-}$states are mainly members of the $\left[2_{1}^{+} \otimes 3_{1}^{-}\right]_{[R P A]}$-quintuplets. Such a state is expected to decay with a large B(E2)-value to the $3_{1}^{-}$state. Only an upper limit of $<35$ W.u. is known for ${ }^{94}$ Mo which is not at variance with this expectation (see Tab. 8.11). Recently in Ref. [Sch10] the $3_{2}^{-}$state of ${ }^{94} \mathrm{Mo}$ at an energy of $3012 \mathrm{keV}$ was claimed to be a candidate for the 
one-phonon octupole mixed-symmetry state. The QPM calculations does not contradict these results since the $\left[3_{3}^{-}\right]_{[R P A]}$-phonons contribute with amplitudes of $\sim 10 \%$ to the $3_{2}^{-}$states in ${ }^{92} \mathrm{Zr}$ an ${ }^{94}$ Mo. Also the $\left[3_{1}^{-}\right]_{[R P A]}$-phonons contribute with similar amplitudes. However, the unperturbed energies of the $\left[3_{3}^{-}\right]_{[R P A]}$-phonons - and hence also the main fragments - are surprisingly high at $4845 \mathrm{keV}, 4788 \mathrm{keV}$ and $4615 \mathrm{keV}$ in ${ }^{94} \mathrm{Mo},{ }^{92} \mathrm{Zr}$ and ${ }^{94} \mathrm{Zr}$ respectively.

\begin{tabular}{|c|c|c|c|c|c|c|}
\hline \multirow[b]{2}{*}{${ }^{\mathrm{A}} X$} & \multirow[b]{2}{*}{$J_{i} \rightarrow J_{f}$} & \multicolumn{3}{|c|}{$\overline{\overline{B(E J)(\text { W.u.) }}}$} & \multicolumn{2}{|c|}{$B(M 1)\left(\mu_{N}^{2}\right)$} \\
\hline & & Exp & $\mathrm{QPM}_{\mathrm{p}}$ & $\mathrm{QPM}_{\mathrm{n}}$ & exp & QPM \\
\hline \multirow{4}{*}{${ }^{94} \mathrm{Mo}$} & $3_{1}^{-} \rightarrow 0_{1}^{+}$ & & 19.7 & 31.5 & & \\
\hline & $3_{2}^{-} \rightarrow 0_{1}^{+}$ & & 2.4 & 3.8 & & \\
\hline & $3_{2}^{-} \rightarrow 3_{1}^{-}$ & $<35$ & 51.6 & & $0.39(7)$ & 0.17 \\
\hline & $5_{1}^{-} \rightarrow 0_{1}^{+}$ & & 6.8 & 6.8 & & \\
\hline \multirow{4}{*}{${ }^{92} \mathrm{Zr}$} & $3_{1}^{-} \rightarrow 0_{1}^{+}$ & & 17.2 & 26.7 & & \\
\hline & $3_{2}^{-} \rightarrow 0_{1}^{+}$ & & 1.4 & 1.7 & & \\
\hline & $3_{2}^{-} \rightarrow 3_{1}^{-}$ & & 20.0 & & & \\
\hline & $5_{1}^{-} \rightarrow 0_{1}^{+}$ & & 5.2 & 3.2 & & \\
\hline \multirow[t]{2}{*}{${ }^{94} \mathrm{Zr}$} & $3_{1}^{-} \rightarrow 0_{1}^{+}$ & & 20.5 & 38.7 & & \\
\hline & $5_{1}^{-} \rightarrow 0_{1}^{+}$ & & 7.5 & 10.2 & & \\
\hline
\end{tabular}

Table 8.11: Same as Tab. 8.3 but for octupole and $5^{-}$states.

The high energy of the unperturbed phonons can be understood in the two-state model. In

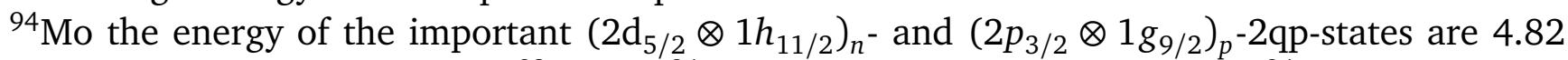
and $4.27 \mathrm{MeV}$ respectively (in ${ }^{92} \mathrm{Zr}$ and ${ }^{94} \mathrm{Zr}$ the $2 \mathrm{qp}$-energies are similar to ${ }^{94} \mathrm{Mo}$ ). Due to the residual octupole proton-neutron interaction both 2qp-states mix and the $3_{1}^{-}$state is pushed below $4.27 \mathrm{MeV}$ while the octupole mixed-symmetry state is above the energy of the $\left(2 d_{5 / 2} \otimes\right.$ $\left.1 h_{11 / 2}\right)_{n}$-2qp-state. In the full QPM calculation this is not entirely true due to the presence of other 2qp-states and the energy of the $\left[3_{3}^{-}\right]_{[R P A]}$-phonon is very close to the $\left(2 d_{5 / 2} \otimes 1 h_{11 / 2}\right)_{n^{-}}$ energy. Nevertheless the high energy of the $\left[3_{3}^{-}\right]_{[R P A]}$-phonon is a shell structure effect and due to the absence of 2qp-states at energies $<3 \mathrm{MeV}$. This is in contrast to the situation for $2^{+}$and $4^{+}$states where the $\left(2 d_{5 / 2} \otimes 2 d_{5 / 2}\right)_{n^{-}}$and $\left(1 g_{9 / 2} \otimes 1 g_{9 / 2}\right)_{p}$-2qp-states are at energies of 1917 $\mathrm{keV}$ and $2698 \mathrm{keV}$.

In the QPM the properties of the 2qp-states are independent of the strength of the residual interaction and are determined by the parameters of the Wood-Saxon potential and the pairing force. These parameters are adjusted to describe the properties of nuclei in this mass region. Therefore, it does not seem likely that the QPM overestimates the 2qp-energies by more than $1 \mathrm{MeV}$. If the QPM calculations are correct the $\left(n, n^{\prime} \gamma\right)$-experiments of Refs. [Fra03, Fra05,Elh08] missed the main fragments of the one-phonon octupole mixed-symmetry states.

It is experimentally demanding to observe it due to the high level densities at these energies. Additionally the presence of two-phonon states - $\left[2_{1}^{+} \otimes 3_{1}^{-}\right]_{[R P A]},\left[2_{1}^{+} \otimes 5_{1}^{-}\right]_{[R P A]},\left[2_{1}^{+} \otimes 4_{1}^{-}\right]_{[R P A]}$, $\left[3_{1}^{-} \otimes 4_{1}^{+}\right]_{[R P A]}$ and $\left[4_{1}^{+} \otimes 5_{1}^{-}\right]_{[R P A]}$ - complicate the situation for $3^{-}$states. The mixed-symmetric phonon can interact with them and might be distributed over many different states. In all three nuclei the $\left[3_{3}^{-}\right]_{[R P A]}$-phonons contribute slightly to the $3_{2}^{-}$states. This is due to a surprisingly large coupling matrix element between the $\left[3_{3}^{-}\right]_{[R P A]}$-phonons and the $\left[2_{1}^{+} \otimes 3_{1}^{-}\right]_{[R P A]^{-}}$ components. 
In Ref. [Sch10] a $J=3$ at $3040 \mathrm{keV}$ is mentioned as a candidate for a $3^{-}$mixed-symmetry state in ${ }^{92} \mathrm{Zr}$. No parity is known for this state. The proton scattering data doesn't give evidence of a $3^{-}$state at this energy. Very close is a known $2^{+}$state at an energy of $3057 \mathrm{keV}$. The energy resolution is not good enough to separate this doublet. In the angular distribution of the cross section, however, is no hint of a contribution from a $3^{-}$state. As shown in Fig. 7.10 it is very well described by a $J^{\pi}=2^{+}$cross section only. This points either to a $3^{+}$state or to a weakly excited $3^{-}$state with a cross section which is smaller than the cross section of any other state in ${ }^{92} \mathrm{Zr}$.

The $3_{1}^{-}$and $3_{2}^{-}$states in ${ }^{94} \mathrm{Mo}$ are connected through an appreciable $B(M 1)$-value of $0.39(7) \mu_{N}^{2}$. Also the QPM predicts a sizable $B(M 1)$-value but underestimates the experimental one by a factor of two. Similarly to the situation of $4^{+}$states a mixed-symmetry state is not mandatory for explaining a large $B(M 1)$-value. It can also be caused by e.g. the fragmentation of the $\left[3_{1}^{-}\right]_{[R P A]}$-phonon and the $\mathrm{B}\left(\mathrm{M} 1:\left[3_{1}^{-}\right]_{[R P A]} \rightarrow\left[3_{1}^{-}\right]_{[R P A]}\right)$ transition. The value of this particular transition depends upon the degree of mixing of the two main 2qp-states and the spin-quenching factor.
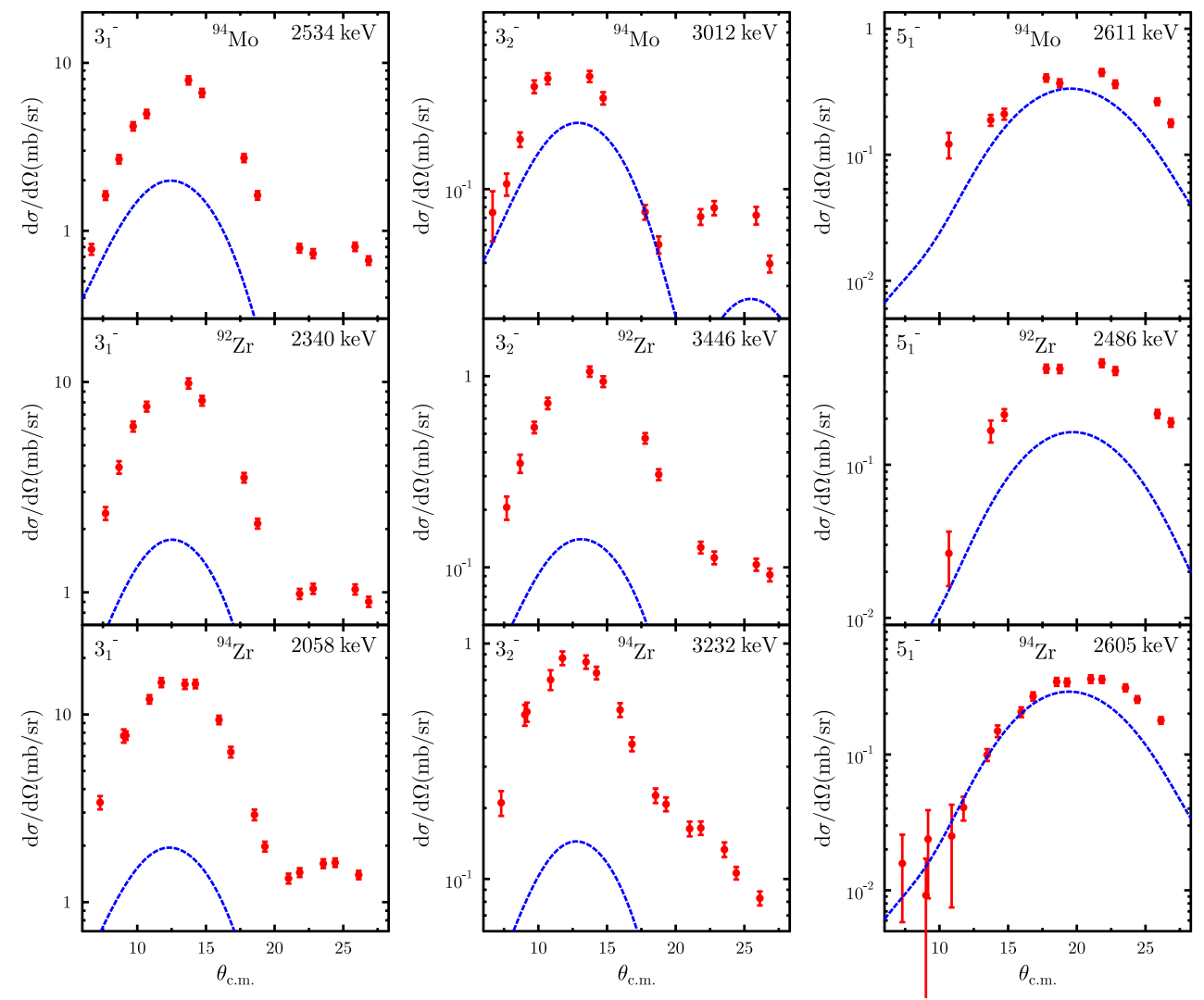

Figure 8.8: Same as Fig. 8.4 but for octupole and $5^{-}$states.

Figure 8.8 compares the experimental proton scattering cross sections to the theoretical ones which are based on the QPM wave functions. The description fails for all six $3^{-}$states. The magnitudes are significantly underestimated sometimes by more than one order of magnitude. Also the shapes do not agree with experiment. This points to a serious shortcoming in the calculation. Surprisingly the electron scattering data shown in Fig. 8.9 are reasonably well described although the $3_{2}^{-}$of ${ }^{94} \mathrm{Mo}$ is overestimated. This clearly shows that the $B(E 3)$-value of the proton part of the wave function is correct (at least its order of magnitude). Hence the 


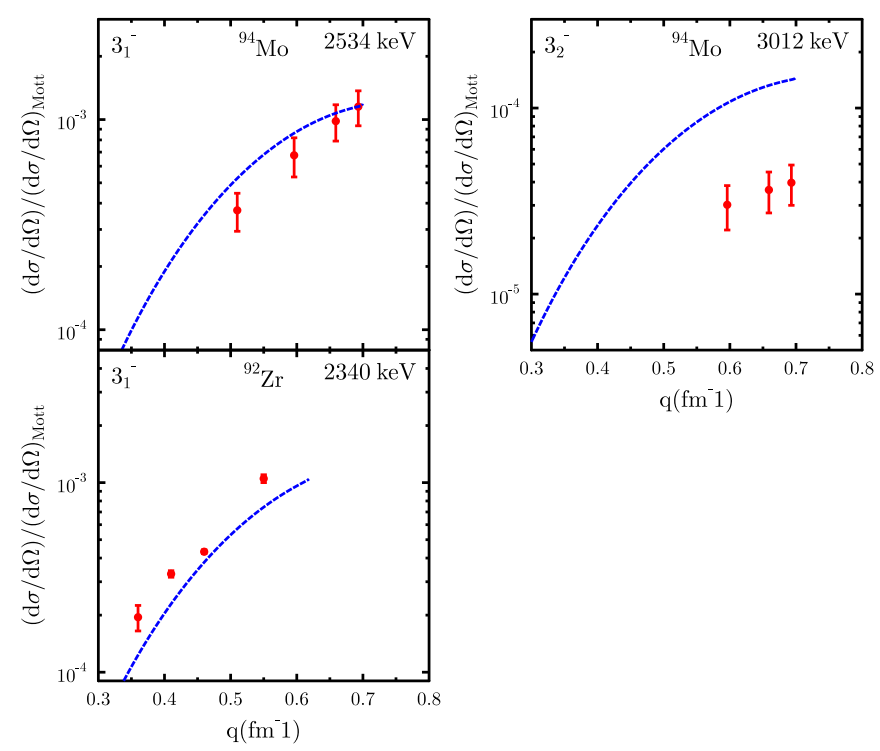

Figure 8.9: Same as Fig. 8.5 but for octupole states.

problem seems to be in the neutron part of the wave functions. The neutron $B(E 3)$-values are given in Tab. 8.11. The one of the $3_{1}^{-}$state of ${ }^{94} \mathrm{Mo}$ amounts to $31.5 \mathrm{~W}$.u.. The proton scattering data suggests that the real experimental value is underestimated by a factor of $\sim 5$ which would set this value to $\sim 160$ W.u.. That is an unreasonable large value which does not agree with the values typically found in this mass region. Hence it seems more likely that the QPM calculations itself are correct and the observed discrepancies are due to the reaction mechanism.

The two-state model does not work for $5^{-}$states. The $\left[5_{1}^{-}\right]_{[R P A]}$-phonon is in all three nuclei a pure $\left(2 p_{1 / 2} \otimes 1 g_{9 / 2}\right)_{p}$-2qp-state which contribute with amplitudes larger than $>93 \%$. In the two-state model one expects two states where the two lowest proton and neutron 2qp-states are in-phase in one state and out-of-phase in the other one. None of the $5^{-}$QRPA-phonons exhibit such a structure. This is possibly due to the large energy difference between the lowest proton and neutron 2qp-states of $1617 \mathrm{keV}$ and the weakness of the residual proton-neutron interaction. The agreement between experimental and theoretical energies shown in Tab. 8.10 is acceptable. The proton scattering cross section in Fig. 8.8 are underestimated by the theoretical calculation for each nucleus. This does not come as a surprise since the QPM does not fulfill the Energy-weighted sum rule (ESWR) for $5^{-}$states.

\subsubsection{Understanding the structure of low-lying natural parity states of ${ }^{92,94} \mathrm{Zr}$ and ${ }^{94} \mathrm{Mo}$}

In summary the ability of the QPM to describe natural parity states in the $\mathrm{N}=50, \mathrm{Z}=40$ region was tested by an extensive comparison to experimental data. The available observables included energies, electromagnetic transition strengths, g-factors and proton and electron scattering cross sections. The investigated nuclei were ${ }^{92,94} \mathrm{Zr}$ and ${ }^{94} \mathrm{Mo}$.

The description of the $2^{+}$states in ${ }^{92} \mathrm{Zr}$ and ${ }^{94} \mathrm{Mo}$ is excellent. Most of the observables are described with a reasonable accuracy giving faith in the QPM wave functions of those states. The most notable discrepancies are the underestimation of the charge transition radii of the $2_{1}^{+}$and $2_{3}^{+}$states in ${ }^{94} \mathrm{Mo}$. Also the predicted energy of the $2_{2}^{+}$state of ${ }^{94} \mathrm{Mo}$ is off by $\sim 400 \mathrm{keV}$. In ${ }^{94} \mathrm{Zr}$ the situation is different. Here it was necessary to lower the energy of the $\left(2 d_{5 / 2} \otimes 2 d_{5 / 2}\right)_{n}$-2qp-state 
artificially by $200 \mathrm{keV}$ in order to achieve a reasonable $B(E 2)$-value of the quadrupole mixedsymmetry state. Additional problems occurred when the interaction between QRPA-phonons is considered which could not be resolved. However the main features of the experimental data are also described on the QRPA level. In order to achieve a better description of ${ }^{94} \mathrm{Zr}$ in the framework of the QPM it seems to be necessary to change the parameters of the WoodSaxon potential which leads to a different single-particle spectrum than in ${ }^{94} \mathrm{Mo}$ and ${ }^{92} \mathrm{Zr}$. Such a treatment is not unreasonable e.g. also in the shell-model the single-particle energies are changed between neighboring nuclei [Sie09]. In general the proton and neutron systems in ${ }^{94} \mathrm{Zr}$ seem to be more separated in energy than in ${ }^{92} \mathrm{Zr}$ and ${ }^{94} \mathrm{Mo}$.

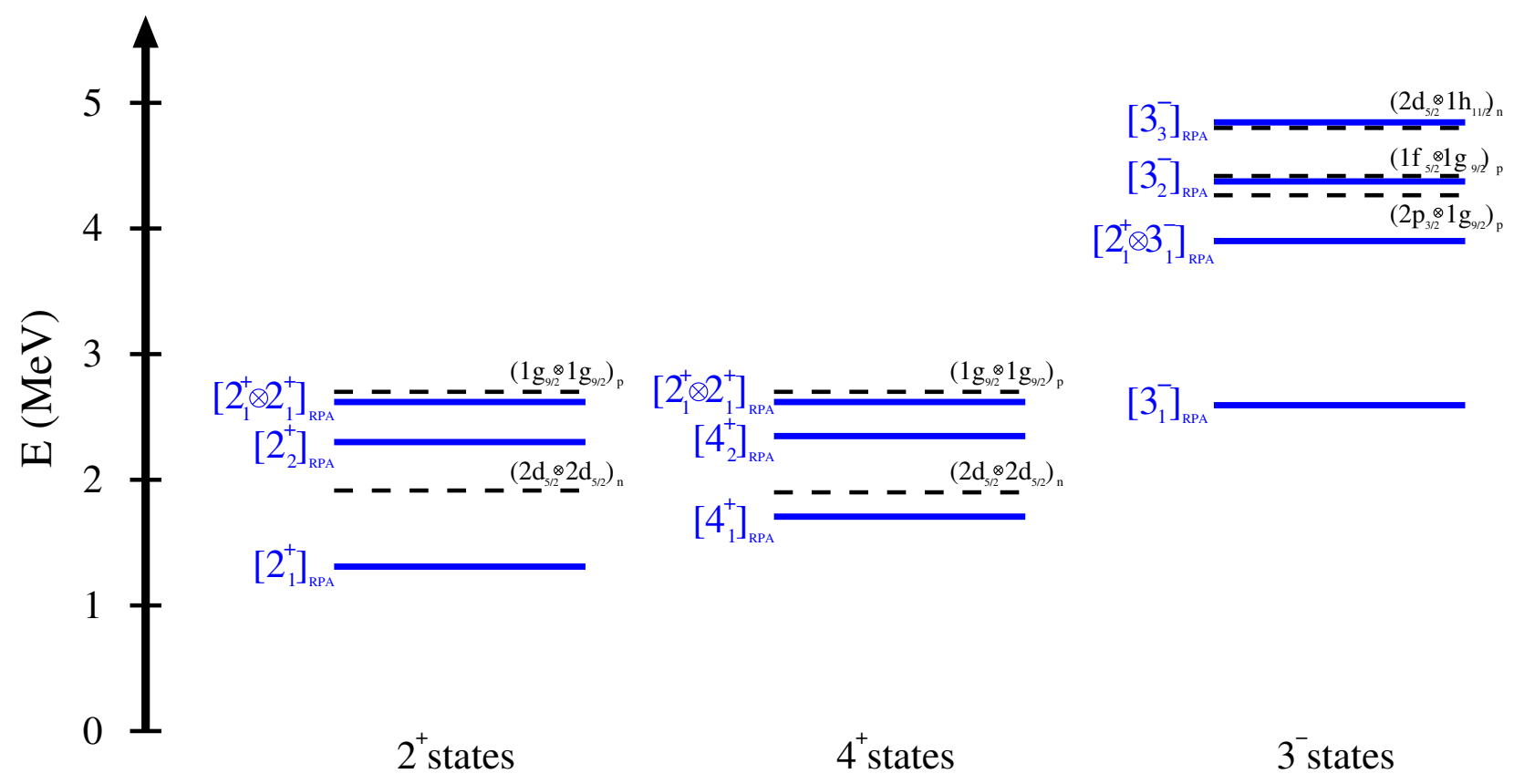

Figure 8.10: Unperturbed phonon (solid blue lines) and 2qp-energies (black dashed lines) of $2^{+}$, $3^{-}$and $4^{+}$states in ${ }^{94} \mathrm{Mo}$.

The QPM has problems in accounting for the properties of the $4^{+}$states. In contrast to the $2^{+}$states the $\left[4_{1}^{+}\right]_{[R P A]^{-}},\left[4_{2}^{+}\right]_{[R P A]^{-}}$and $\left[2_{1}^{+} \otimes 2_{1}^{+}\right]_{[R P A]}$-phonons are close in energy which makes the results very sensitive to details of the calculations. This fact is highlighted in Fig. 8.10 which compares the unperturbed phonon energies of the $2^{+}$and $4^{+}$phonons for ${ }^{94} \mathrm{Mo}$. The QPM is not able to achieve a good description of all observables simultaneously. Nevertheless some features of the data are well described by the QPM.

For $3^{-}$and $5^{-}$states less experimental data is available. The observables which are available are reproduced by QPM except for the proton scattering cross sections of the $3^{-}$states. All six experimental cross sections are underestimated in the calculation sometimes by more than an order of magnitude. There are indications that these deviations are not due to problems with the QPM itself but because of a mistake when calculating the proton scattering cross sections from the QPM wave functions. However at the moment it was not possible to resolve this problem and the QPM wave functions of the $3^{-}$have to be treated with care.

Nevertheless the QPM is successful in accounting for most of the features of the exhaustive experimental data. Therefore it is possible to obtain an understanding in the framework of this model which degrees of freedom are important for low-lying natural parity states in the $N=50$, $Z=40$ mass region and their mutual interplay. 
The fundamental degrees of freedom of the lowest states of each multipolarity are the symmetric and mixed-symmetric QRPA-phonons and two-phonon states - namely the $\left[2_{1}^{+} \otimes 2_{1}^{+}\right]_{[R P A]}^{2^{+}}$, $\left[2_{1}^{+} \otimes 2_{1}^{+}\right]_{[R P A]}^{4^{+}}$and $\left[2_{1}^{+} \otimes 3_{1}^{-}\right]_{[R P A]}^{3^{-}}$- which can be formed by coupling two symmetric QRPAphonons. A key point is the simple two-state model which explains the formation mechanism of the symmetric and mixed-symmetric QRPA-phonons. For each multipolarity (expect for the $5^{-}$states) the lowest proton 2qp-state is mixed with the lowest neutron 2qp-state. Two states are formed, one where both are connected with a plus sign - the counterpart of the symmetric QRPA-phonons - and one where both are connected with a minus sign - the counterpart of the mixed-symmetric QRPA-phonon. A comparison with QRPA calculation reveal that indeed a large fraction of the wave functions can be understood in this simple picture.

The different strengths of the residual interactions explain the different degrees of mixing of the two important 2qp-states for each multipolarity. The energy difference between the two important 2qp-states is the same for $2^{+}$and $4^{+}$states. The hexadecapole proton-neutron residual interaction is known to be weaker than the quadrupole proton-neutron residual interaction. This leads to a strong mixing of the $\left(2 d_{5 / 2} \otimes 2 d_{5 / 2}\right)_{n^{-}}$and $\left(2 p_{1 / 2} \otimes 1 g_{9 / 2}\right)_{p}$-2qp-states in case of the $2^{+}$states and to almost pure 2qp-states in case of the $4^{+}$states.

Figure 8.10 shows the unperturbed phonon energies of the $2^{+}, 4^{+}$and $3^{-}$states for ${ }^{94} \mathrm{Mo}$ (solid blue lines) and the two 2qp-states which are important for each multipolarity (dashed black lines). Due to the different strengths of the quadrupole and hexadecapole residual interactions the $\left[2_{1}^{+}\right]_{[R P A]}$-phonon is pushed further away from the $\left[2_{2}^{+}\right]_{[R P A]^{-}}$and $\left[2_{1}^{+} \otimes 2_{1}^{+}\right]_{[R P A]^{-}}^{2^{+}}$ phonons than the $\left[4_{1}^{+}\right]_{[R P A]}$-phonon from the $\left[4_{2}^{+}\right]_{[R P A]}$ - and $\left[2_{1}^{+} \otimes 2_{1}^{+}\right]_{[R P A]}^{4^{+}}$-phonons. This leads two a strong phonon mixing in case of the $4^{+}$states while for the $2^{+}$states one QRPA-phonon forms essentially one final QPM state. Due to the fragmentation of the hexadecapole one- and two-phonon states a large B(M1)-value is no longer a unique and reliable signature to identify a mixed-symmetry state. The diagonal M1-matrix elements (e.g. of the $\left[4_{2}^{+}\right]_{[R P A]}$-phonon) can also cause large B(M1)-values which have a similar size than the one connecting symmetric and mixed-symmetric phonons. In order to identify a hexadecapole mixed-symmetry state a nuclear structure model is needed which is able to account for the properties of the low-lying $4^{+}$states. Unfortunately the QPM is not accurate enough to allow such an interpretation and to answer the question whether the $4_{2}^{+}$states in ${ }^{92} \mathrm{Zr}$ and ${ }^{94}$ Mo have large mixed-symmetric components.

The QPM results suggest that an experimental identification of the octupole one-phonon mixed-symmetry state is very difficult. Due to the absence of 2qp-states at low energies the mixed-symmetric QRPA-phonon is in all three nuclei at energies $>4 \mathrm{MeV}$ (see Fig. 8.10). The two lowest $3^{-}$states are formed by the $\left[3_{1}^{-}\right]_{[R P A]^{-}}$and $\left[2_{1}^{+} \otimes 3_{1}^{-}\right]_{[R P A]}^{3^{-}}$-phonons.

The symmetric and mixed-symmetric quadrupole phonons can also form a mixed-symmetric two-phonon state $\left[2_{1}^{+} \otimes 2_{1}^{2}\right]_{[R P A]}^{2^{+}}$. In ${ }^{94} \mathrm{Mo}$ the $2_{5}^{+}$state at $2870 \mathrm{keV}$ was identified as the mixedsymmetric two-phonon state because of its large $B(M 1)$-value to the symmetric two-phonon state [Fra03]. Furthermore the authors of Ref. [Bur07] used the same proton scattering data which was also analyzed in this thesis to prove the two-phonon character of this state. This was done by investigating the angular distribution of the cross section and it was claimed that this cross section can only be explained by the inclusion of a multi-step excitation mechanism through the $2_{1}^{+}$and $2_{3}^{+}$states. However there is an alternative way to explain the deviations of the cross section from the expectations for a one-phonon state. A $6^{+}$state - which was known at the time when the publication of Ref. [Bur07] was written - is only $2.4 \mathrm{keV}$ way from the $2_{5}^{+}$ state. Clearly, this doublet cannot be separated in a proton scattering experiment. Figure 8.11 


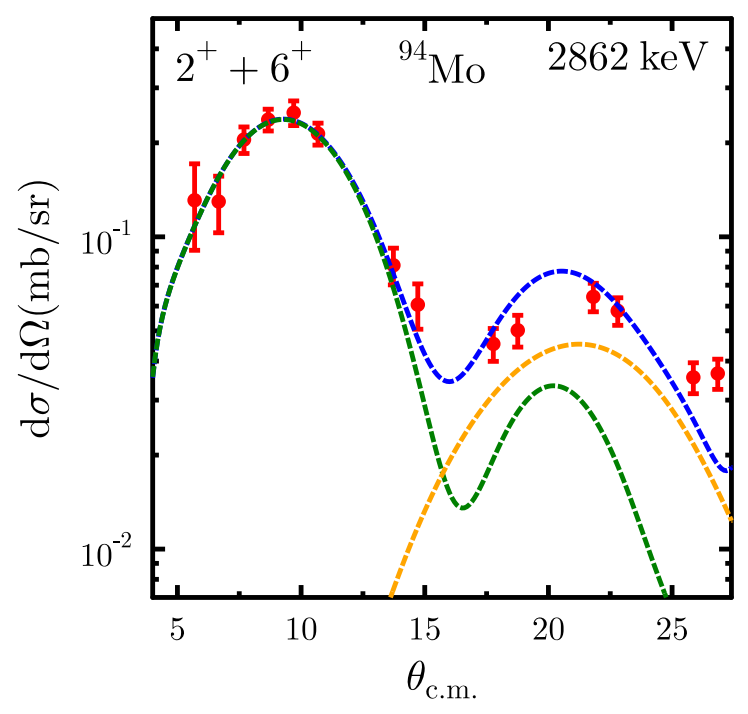

Figure 8.11: Cross section of the $2^{+}$and $6^{+}$doublet at $2862 \mathrm{keV}$ in ${ }^{94} \mathrm{Mo}$. The cross section is well described by a superposition (dashed blue line) of a $2^{+}$(dashed green line) and $6^{+}$(dashed yellow line) one-step cross section.

compares the proton scattering data of the $2_{5}^{+}$state to a theoretical cross section assuming a superposition of a one-step $2^{+}$and $6^{+}$cross sections. The calculations were performed with the computer code CHUCK3. The description is excellent and better than the one assuming a multi-step excitation mechanism. This clearly contradicts the result of Ref. [Bur07]. The data does not give evidence of the two-phonon nature of this state. The author's have overseen the contributions of the $6^{+}$state. However it is still possible that this state has a large two-phonon component. But there is clearly a significant one-phonon component in the wave function determining the excitation mechanism in proton scattering. 


\subsection{Proton and neutron transition matrix elements to the ground state: Sensitivity to mixed-symmetry states and origin of collectivity}

A large $B(M 1)$-value to the symmetric one-phonon state is an ambiguous signature to identify a mixed-symmetry state in cases where the fragmentation of QRPA-phonons is important. Furthermore it is desirable to have more than just one experimental observable at hand which is sensitive to the special character of a mixed-symmetry state, in order to investigate its properties. In general theoretical nuclear wave functions are more stringently tested if they are compared to as many observables as possible.

This section has three purposes. Firstly it investigates the capability of the quantity $R$ - defined in Eq. 8.1 - to identify a mixed-symmetry state if a priori nothing is known about its excitation energy. Secondly the sensitivity of absolute proton and neutron transition matrix elements to the mixed-symmetry character is discussed. Lastly this section provides insights on the formation mechanism of low-lying collective states (i.e. states which have large matrix elements to the ground state) which are also partly reported by the authors of Refs. [Sag87, Iud04]. All three points are closely related to each other.

The authors of Ref. [Leo98, Leo89, Pig88] used absolute proton and neutron transition matrix elements to evaluate the quantity $R$

$$
R=\left|\frac{M_{n}-M_{p}}{M_{n}+M_{p}}\right| .
$$

$M_{\mathrm{p}}$ and $M_{\mathrm{n}}$ are the proton and neutron transition matrix elements to the ground state. They look for enhanced values of $R$ and identify the corresponding state as a mixed-symmetry state. For the nuclei ${ }^{92,94} \mathrm{Zr}$ and ${ }^{94} \mathrm{Mo}$ a complete set of proton and neutron $B(E 2)$-values is available. From $B(M 1)$-values it is well known which $2^{+}$state is the mixed-symmetry candidate offering the possibility to investigate the capability of $R$ to identify members of this class of states.

\begin{tabular}{c|cc|cc|cc}
\hline \hline & \multicolumn{2}{|c|}{${ }^{94} \mathrm{Mo}$} & \multicolumn{2}{c|}{${ }^{92} \mathrm{Zr}$} & \multicolumn{2}{c}{${ }^{94} \mathrm{Zr}$} \\
& $2_{1}^{+}$ & $2_{\mathrm{ms}}^{+}$ & $2_{1}^{+}$ & $2_{\mathrm{ms}}^{+}$ & $2_{1}^{+}$ & $2_{\mathrm{ms}}^{+}$ \\
\hline full & 0.16 & 0.02 & 0.21 & 0.08 & 0.27 & 0.14 \\
valence & 0.03 & 6.16 & 0.39 & 14.67 & 0.37 & 4.72 \\
\hline \hline
\end{tabular}

Table 8.12: QRPA results for the $R$-value defined in Eq. 8.1 using the full QPM model space ('full' ) and only the two main components ('valence' ).

In the two-state model introduced in Sect. 8.1 one expects for the $2_{1}^{+}$state a value $R<1$ due to the cancellation of proton an neutron matrix elements in the nominator (depending upon how similar $M_{\mathrm{n}}$ and $M_{\mathrm{p}}$ are). For the mixed-symmetry state both matrix elements add coherently in the nominator and cancel in the denominator leading to $R>1$. The QPM results - considering the two main neutron and proton components only - are shown in Tab. 8.12 in the row labeled 'valence'. Clearly, $R$ of the mixed-symmetry state is enhanced in comparison to $R$ of the $2_{1}^{+}$state. Moreover it is significantly larger than 1 which allows possibly a differentiation from other noncollective states of the same multipolarity. For some of the non-collective states one can expect a dominant proton or neutron 2-qp component leading to $R \approx 1$ (a pure 2qp-state has exactly $R=1$ ). At first glance $R$ seems to be a promising quantity. The crucial point is, however, to 
which degree other 2qp-states - which are not considered in the two-state model but contribute to the QRPA wave functions - influence the final value of $R$.

The proton and neutron transition matrix elements of the QPM were tested in the last sections by a comparison to experimental data. Due to the good agreement in case of the quadrupole states, the following analysis is done based on the QPM matrix elements given in Tab. 8.3. The $R$-values of the full QRPA calculation are displayed in Tab. 8.12 in the row labeled 'full' . The values of the $2_{1}^{+}$states follow the expectations. Surprisingly the $R$-values of the mixed-symmetry states are not only smaller than one, they are even smaller than the $R$-values of the $2_{1}^{+}$states.

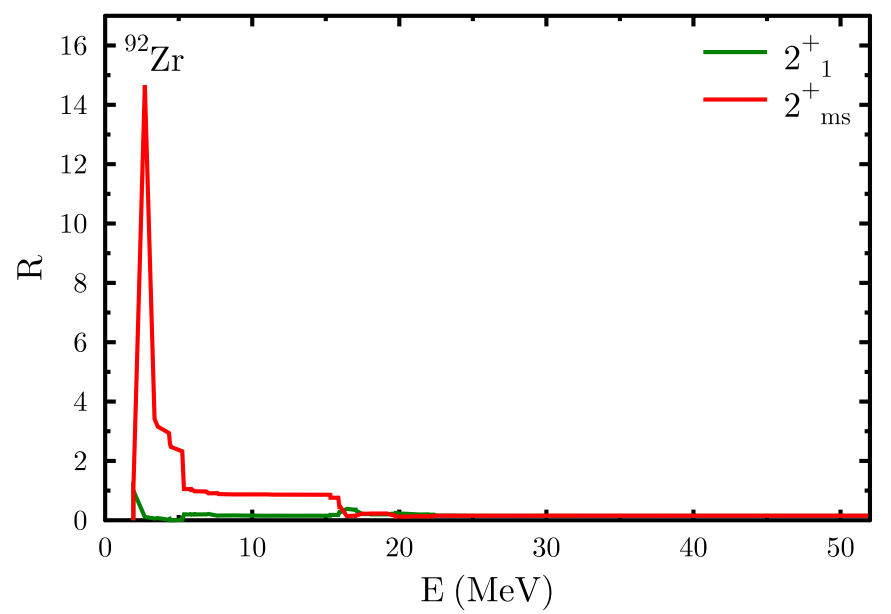

Figure 8.12: Running sum of the $R$-value defined in Eq. 8.1 of the $\left[2_{1}^{+}\right]_{[R P A]^{-}}$and $\left[2_{\mathrm{ms}}^{+}\right]_{[R P A]^{-}}$ phonons in ${ }^{92} Z$. Due to the contributions from high-lying 2qp-states in the energy range of $15-25 \mathrm{MeV}$ the $R$-value is not sensitive to the valence space structure.

In order to understand this unexpected behavior, $R\left(2_{1}^{+}\right)$and $R\left(2_{\mathrm{ms}}^{+}\right)$of ${ }^{92} \mathrm{Zr}$ are decomposed in the contributions of the various 2qp-states in Fig. 8.12. In this figure the energy E acts like a threshold. All 2-qp states below this energy are included in the calculation of $R$ and all above are not considered. The value of $R\left(2_{1}^{+}\right)$drops quickly to $R \approx 0$. The large value of $R\left(2_{\mathrm{ms}}^{+}\right)$when only the two main components are considered - is already significantly reduced by other 2qp-states with energies $<5 \mathrm{MeV}$. Between 5 and $15 \mathrm{MeV}$ it stays rather constant with $R\left(2_{\mathrm{ms}}^{+}\right) \approx 1$ and drops eventually to $R \approx 0$.

Further insights can be obtained from the same kind of decomposition for the neutron and proton $B(E 2)$-values shown in the upper part of Fig. 8.13. For comparison the non-collective $\left[2_{3}^{+}\right]_{[R P A]}$-phonon is also presented. A significant part of the $B(E 2)$-values of the collective states stems from 2qp-states in the energy region $15-25 \mathrm{MeV}$. Those are the same 2qp-states which cause the drop of the $R\left(2_{\mathrm{ms}}^{+}\right)$-value from $R \approx 1$ to $R \ll 0$ in Fig. 8.12. Due to their contributions to the wave functions the sensitivity of $R$ to the valence structure - in particular to the minus sign connecting the two main components - is reduced. In nuclei where the mixed-symmetry state is less collective, i.e it has less contributions from high-lying 2qp-states, the situation is possibly different and $R$ might be more sensitive to the valence part of the wave function. This is the case for the non-collective $\left[2_{3}^{+}\right]_{[R P A]}$-phonon which is almost a pure 2qp-state and has small contributions from high-lying 2qp-states. Here $\mathrm{R}$ is close to one indicating a pure 2qp-state. In ${ }^{92} \mathrm{Zr}$, however, the large $R$-value of the two main components is already significantly altered by other low-lying 2qp-states with energies $<5 \mathrm{MeV}$. Therefore $R$ cannot be considered as a good 
signature to identify a mixed-symmetry state and the results of Refs. [Leo89, Leo98, Pig88] are questioned.

Nevertheless it is still a good idea to test the wave function of a known mixed-symmetry state with a comparison to proton and neutron $B(E 2)$-values. The sensitivity to the main components is not entirely lost. For example the total neutron transition matrix element of the mixedsymmetric QRPA-phonon of ${ }^{94}$ Mo amounts to 5.93 ef $\mathrm{m}^{2}$ while the contribution of the $\left(2 d_{5 / 2} \otimes\right.$ $\left.2 d_{5 / 2}\right)_{n}$-2qp-state is -3.43 ef $\mathrm{m}^{2}$ i.e. the main component still has a pronounced influence on the total neutron $B(E 2)$-value. Consequently a successful description of absolute proton and electron scattering cross sections like in Sect. 8.1 is also an indication that the model predicts the properties of the main components correctly.

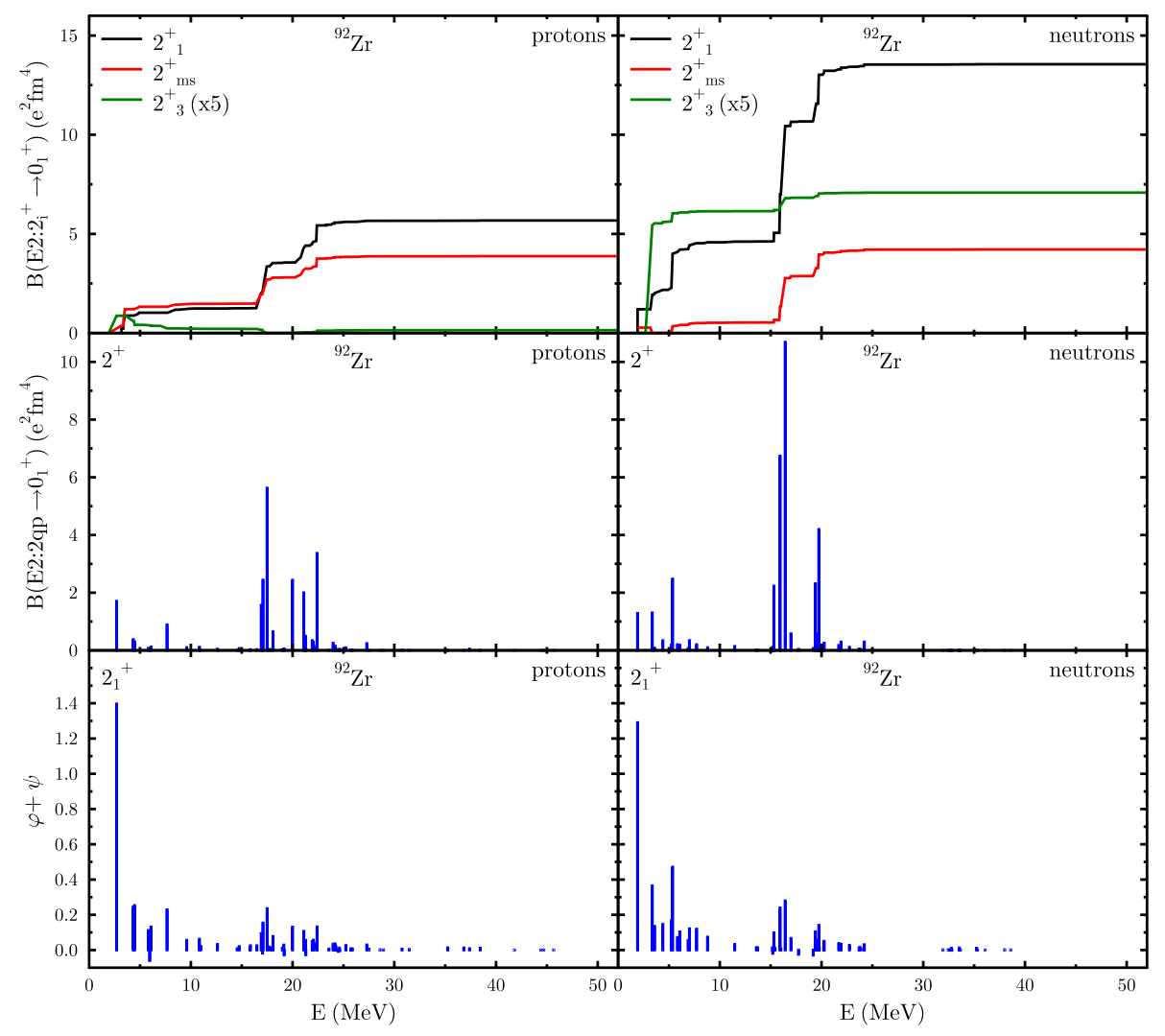

Figure 8.13: Running sums of the $B(E 2)$-values of the $\left[2_{1}^{+}\right]_{[R P A]^{-}}\left[2_{\mathrm{ms}}^{+}\right]_{[R P A]^{-}}$and $\left[2_{3}^{+}\right]_{[R P A]^{-}}{ } \mathrm{QRPA}$ phonons of ${ }^{92} \mathrm{Zr}$. The $\left[2_{3}^{+}\right]_{[R P A]}$-phonon is a non-collective (top). $B(E 2)$-values of the various 2-qp states (middle). Sum of forward $(\psi)$ and backward amplitudes $(\varphi)$ of each 2-qp state for the $\left[2_{1}^{+}\right]_{[R P A]}$-phonon (bottom).

Although the quantity $R$ does not constitute a good observable to identify mixed-symmetry states, the theoretical analysis presented in this section provides insights in the origin of collectivity in vibrational nuclei. This important point is further discussed in the following. At first glance the large contribution of high-lying 2qp-state to the $B(E 2)$-values comes as a surprise since their contribution to the QRPA-norm of the wave functions (Eq. 5.32) is typically less than $1 \%$.

In the middle of Fig. 8.13 the proton and neutron $B(E 2)$-values of each 2qp-state are displayed. The $B(E 2)$-values in the region of $15-25 \mathrm{MeV}$ are clearly enhanced in comparison to 
the $B(E 2)$-values in the valence space. These 2qp-states are also responsible for the formation of the Giant Quadrupole Resonance (GQR) which can be found at similar energies [Pon14]. The particle-hole residual interaction mixes them into the wave functions of the $2_{1}^{+}$and $2_{\mathrm{ms}}^{+}$ states causing their collective $B(E 2)$-values of several W.u.. Hence their small forward and backward amplitudes $\psi_{j j^{\prime}}, \varphi_{j j^{\prime}}$ in Eq. 5.34 are compensated by large single-particle transition matrix elements $u_{j j^{\prime}}^{(+)} \cdot\left\langle j\|E \lambda\| j^{\prime}\right\rangle$.

In order to understand why these matrix elements are so drastically enhanced Fig. 8.14 shows on the left-hand side the radial parts of the single-particle wave functions that form the 2qp-state $\left(1 g_{9 / 2} \otimes 1 i_{11 / 2}\right)_{n}$ which has the largest $B(E 2)$-value. For comparison the radial single-particle wave functions of the $\left(2 s_{1 / 2} \otimes 2 d_{5 / 2}\right)_{n}$-2qp-state are also shown. This 2qp-state has a noncollective $B(E 2)$-value. According to Eq. 8.2 a large E2-transition matrix element can be caused by a good spatial overlap of the two single-particle wave functions,

$$
\left\langle j\|E \lambda\| j^{\prime}\right\rangle_{s} \sim \int_{0}^{\infty} \varphi_{j}(r) \cdot r^{\lambda+2} \cdot \varphi_{j^{\prime}}(r) \cdot d r .
$$

Here, $\varphi_{i}$ are the single-particle wave functions and $\lambda$ is the multipolarity of the transition. Due to the factor $r^{\lambda+2}$ this good overlap is particularly important at larger distances to the center of mass of the nucleus and favors single-particle states with large $l$ quantum numbers, since they are located at larger $r$ because of the 'centrifugal repulsion' . Indeed the $1 g_{9 / 2}$ and $1 h_{11 / 2}$ singleparticle wave functions have both large $l$ quantum numbers and a good spatial overlap causing this very large matrix element. Similar observations can be made for the other enhanced $B(E 2)$ values in the energy region $15-25 \mathrm{MeV}$. In contrast the $2 s_{1 / 2}$ and $2 d_{5 / 2}$ single-particle states have smaller $l$ quantum numbers and a reduced spatial overlap. To further illustrate this point the radial dependence of the integrand of Eq. 8.2 is shown on the right hand side of Fig. 8.14. As expected the one of the $1 g_{9 / 2}$ and $1 h_{11 / 2}$ single-particle states is significantly enhanced in the surface region $(r \approx 6 \mathrm{fm})$ in comparison to the one of the $2 s_{1 / 2}$ and $2 d_{5 / 2}$ single-particle states.
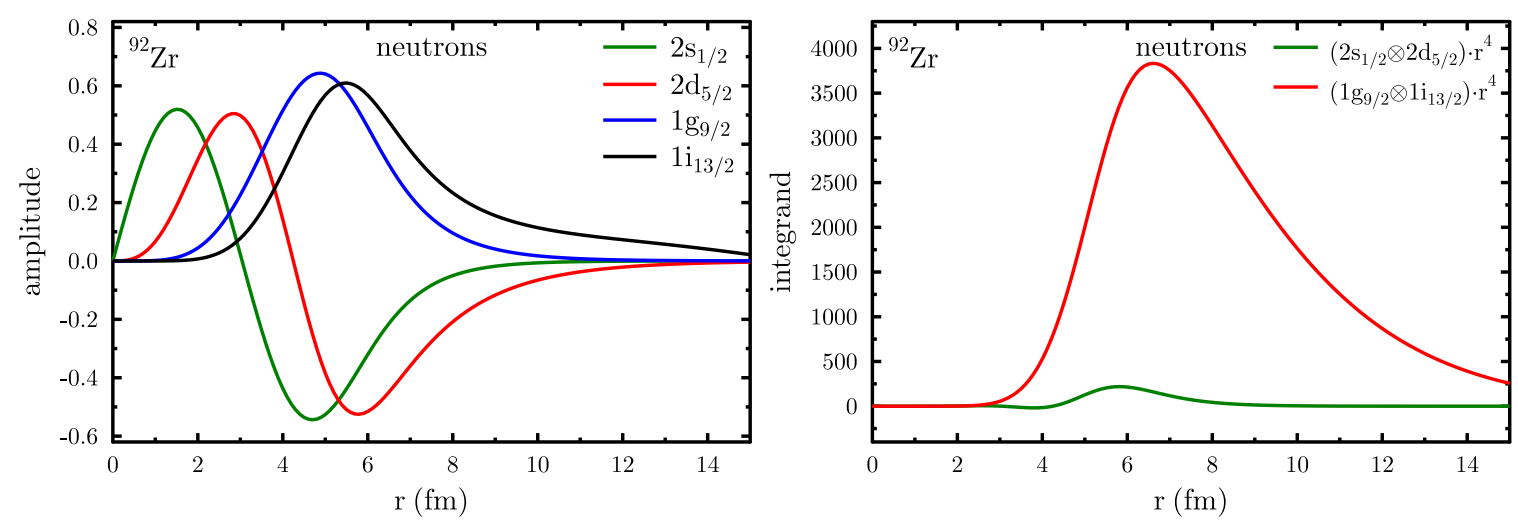

Figure 8.14: Radial wave functions of four neutron single-particle states (left-hand side). On the right hand side the radial dependence of the integrand of Eq. 8.2 for $\lambda=2$ is shown. Wave functions which have good overlaps and high I values produce large E2-matrix elements.

Also surprising is the fact that all 2qp-states with large $B(E 2)$-values are confined in a $10 \mathrm{MeV}$ wide energy window. At smaller and larger energies none of them can be found. According to Eq. 5.34 two conditions have to be fulfilled that a 2qp-state has a large B(E2)-value. Besides the 
already discussed single-particle transition matrix element $\left\langle j\|E \lambda\| j^{\prime}\right\rangle$ the factor $u_{j j^{\prime}}^{(+)}=u_{j} v_{j}^{\prime}+$ $u_{j}^{\prime} v_{j}$ is important. The squares of the Bogoljubov coefficients $u$ and $v$ represent the probabilities that a single-particle state is either occupied or empty. Due to this factor the $B(E 2)$-values of particle-hole transitions are enhanced (e.g. $u_{j} \approx 1, v_{j} \approx 0, u_{j^{\prime}} \approx 0, v_{j^{\prime}} \approx 1$ ) while the $B(E 2)$ values of particle-particle transitions are strongly suppressed because $u_{j j^{\prime}}^{(+)} \approx 0$ (e.g. $u_{j} \approx 1$, $v_{j} \approx 0, u_{j^{\prime}} \approx 1, v_{j^{\prime}} \approx 0$ ).

All single-particle states within one major shell have the same parity except the unique parity single-particle state which is pushed down from the next oscillator shell because of the spin-orbit force. In order to create a 2qp-state with positive parity two single-particle states are needed which are of the same harmonic oscillator shell $(0 \hbar \omega)$, differ by two harmonic oscillator shells $(2 \hbar \omega)$ or by four harmonic oscillator shells $(4 \hbar \omega)$. $1 \hbar \omega$ and $3 \hbar \omega$ transitions cannot form a 2qp-state with positive parity except with the unique parity state. All $n \hbar \omega$ transitions (with non-zero $u_{j j^{\prime}}^{(+)}$) fall approximately in the same energy range. At low energies are $0 \hbar \omega$ transitions of the harmonic oscillator shell which is close to the Fermi surface. The involved single-particle states are able to produce large single-particle matrix elements like the diagonal matrix element of the $1 g_{9 / 2}$ state. However they are never so enhanced like in the $15-25 \mathrm{MeV}$ region because no single-particle states with $l>4$ contribute to this harmonic oscillator shell. The large $B(E 2)$ value in the $15-25 \mathrm{MeV}$ range are $2 \hbar \omega$ transitions with large $l$ quantum numbers like the $\left(1 f_{7 / 2} \otimes\right.$ $\left.1 h_{11 / 2}\right)_{n},\left(1 g_{9 / 2} \otimes 1 i_{13 / 2}\right)_{n}$ - and $\left(1 f_{5 / 2} \otimes 1 h_{9 / 2}\right)_{n}$-2qp-states.

From 10 to $15 \mathrm{MeV}$ is the typical energy range of $1 \hbar \omega$ transitions which cannot contribute. The only possibility that a 2qp-state with positive parity falls in this region is an involvement of the unique parity single-particle states. Since these single-particle state have typically large $j$ quantum number only a few other single-particle states have a sufficiently high $j$ to couple with it to an angular momentum of $J=2$. Hence the 'gap' between $0 \hbar \omega$ and $2 \hbar \omega$ transitions in Fig. 8.13 is formed. The same holds at higher energies for $3 \hbar \omega$ transitions. In general only a few $4 \hbar \omega$ transition show up in the 2qp-spectrum of the QPM. Therefore also the region from 30 to $40 \mathrm{MeV}$ has almost no sizable $B(E 2)$-values.

Besides the large single-particle matrix elements there is a second reason why the high-lying 2qp-states have a strong influence on the $B(E 2)$-values of the $2_{1}^{+}$and $2_{\mathrm{ms}}^{+}$states. The high-lying 2qp-states have similar forward and backward amplitudes. Hence their contribution to the norm of Eq. 5.32 is small because both cancel. On the other hand the expression for the E2-matrix element given in Eq. 5.34 depends on the sum of the forward and backward amplitudes of each 2qp-state. In the bottom part of Fig. 8.13 this sum $\psi_{j j^{\prime}}+\varphi_{j j^{\prime}}$ is shown for each 2qp-state $\left(j \otimes j^{\prime}\right)$ for the proton and neutron $B(E 2)$-values of the $2_{1}^{+}$state. The ratio of $\psi_{j j^{\prime}}+\varphi_{j j^{\prime}}$ of the dominant valence 2qp-states and the high-lying 2qp-states is more balanced than for the norm.

It should be noted that the $B(E 2)$-strengths and $\psi_{j j^{\prime}}+\varphi_{j j^{\prime}}$ distributions of the 2qp-states exhibit some similarities. Two-quasiparticle states with large $B(E 2)$-values have usually also sizable $\psi_{j j^{\prime}}+\varphi_{j j^{\prime}}$ values. For example the distribution of $\psi_{j j^{\prime}}+\varphi_{j j^{\prime}}$ in the $15-25 \mathrm{MeV}$ range is very similar to the distribution of $B(E 2)$-values in this region. However it was not possible to find a quantitative explanation for this behavior.

Figures 8.15 and 8.16 display the same kind of decomposition for $4^{+}$and $3^{-}$states, respectively. Again, a large portion of the $B(E \lambda)$-values of the $\left[4_{1}^{+}\right]_{[R P A]^{-}},\left[4_{\mathrm{ms}}^{+}\right]_{[R P A]^{-}}$and $\left[3_{1}^{-}\right]_{[R P A]^{-}}$ phonons is caused by 2qp-states that are not part of the valence space. In contrast, the $\left[3_{\mathrm{ms}}^{-}\right]_{[R P A]}$-phonon is non-collective and its $B(E 3)$-values is determined by the two main valence components. The 2qp-states with large B(E3)-values are shown in the bottom part of 


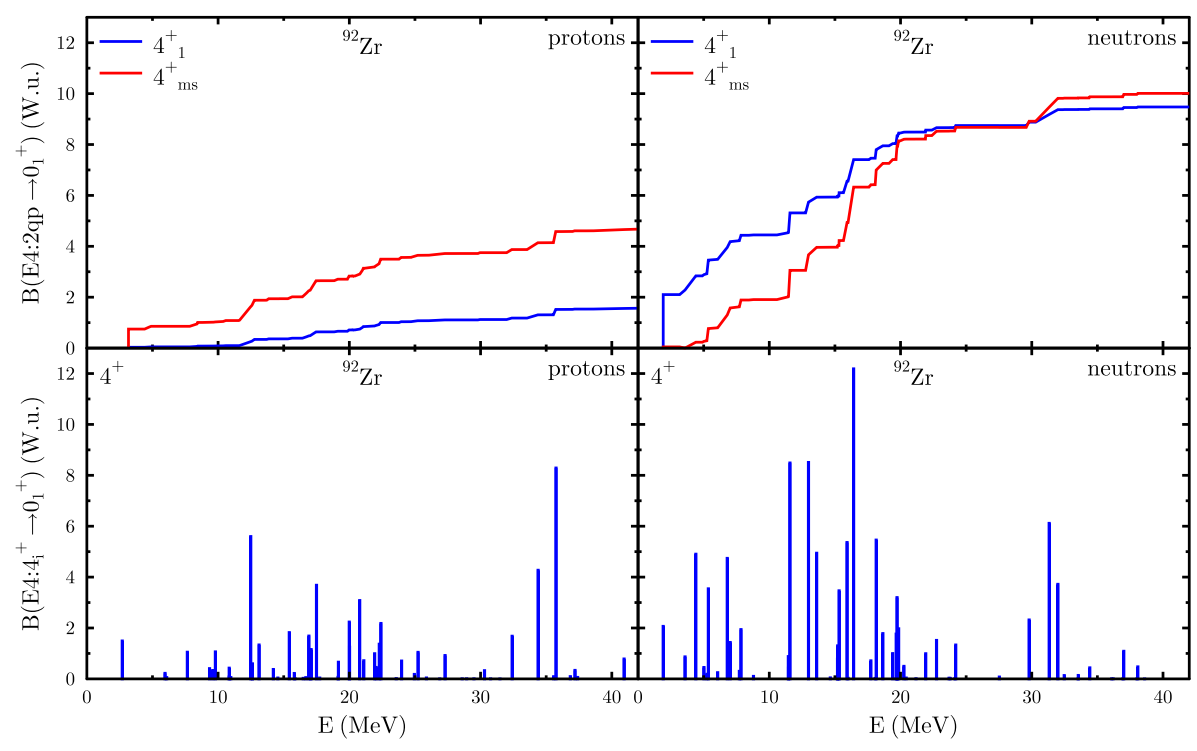

Figure 8.15: Same like Fig. 8.13 for hexadecapole states.

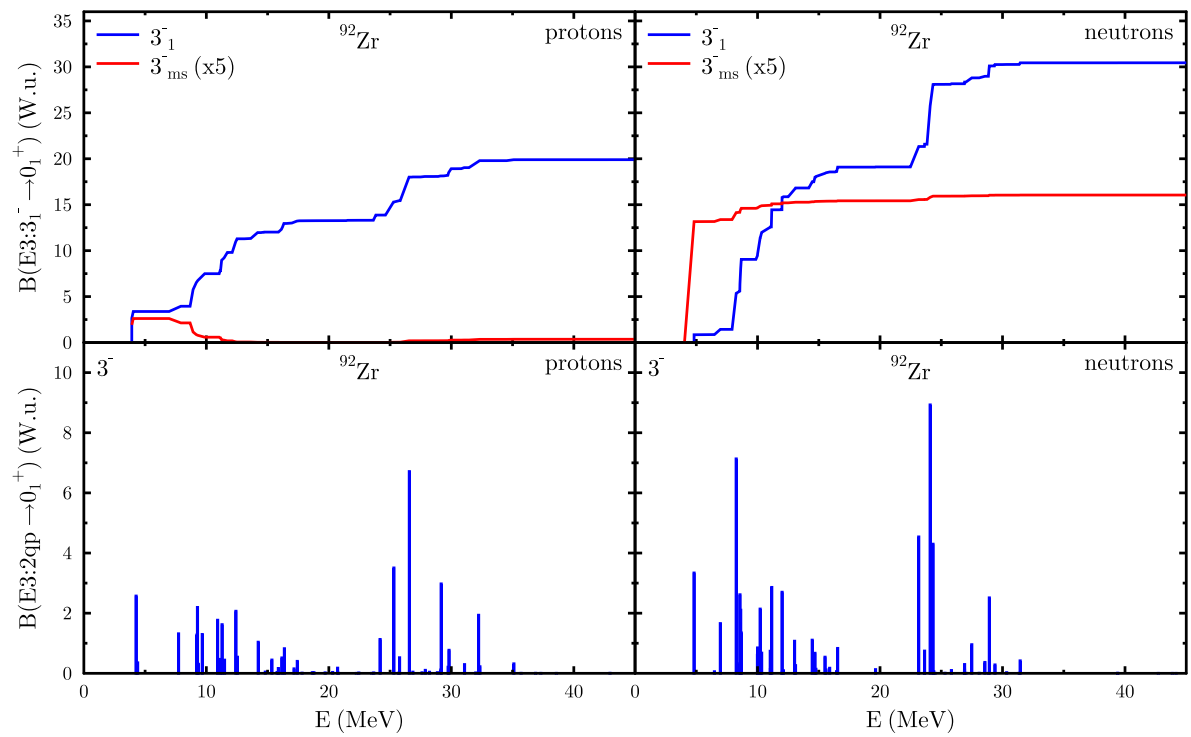

Figure 8.16: Same like Fig. 8.13 for octupole state.

Fig. 8.16. The 2qp-states that can couple to $J^{\pi}=3^{-}$have to be $1 \hbar \omega$ or $3 \hbar \omega$ transitions explaining the two clusters of $B(E 3)$-values at 5-15 MeV and 25-30 MeV respectively. Those are approximately the energy regions that were empty for the $2^{+}$states.

The $B(E 4)$-values of hexadecapole states are not grouped in clusters like $2^{+}$and $3^{-}$states and are distributed over a wide energy range. In particular in the $1 \hbar \omega$ region hexadecapole 2qp-states occur. A unique parity single-particle state contributes to most of these 2qp-states like the $1 h_{11 / 2}$ and $1 i_{13 / 2}$ states. Many of the single-particle states of the next lower major shell can couple with these unique parity single-particle states to $J=4$ but not $J=2$ explaining why the ' $1 \hbar \omega$ gap' is filled for $4^{+}$states but not for $2^{+}$states. Similar observations are reported in Ref. [Geo82]. 


\subsection{New signature of one-phonon quadrupole mixed-symmetry states}

It is the purpose of this section to introduce a new experimental signature which gives evidence of the mixed-symmetric character of the $2_{2}^{+}$states in ${ }^{92,94} \mathrm{Zr}$ and the $2_{3}^{+}$state in ${ }^{94} \mathrm{Mo}$ independently of absolute electromagnetic transition strengths. This new observable proves directly the minus sign of the $\left(2 d_{5 / 2} \otimes 2 d_{5 / 2}\right)_{n}$-component in the wave function of the mixed-symmetry state.
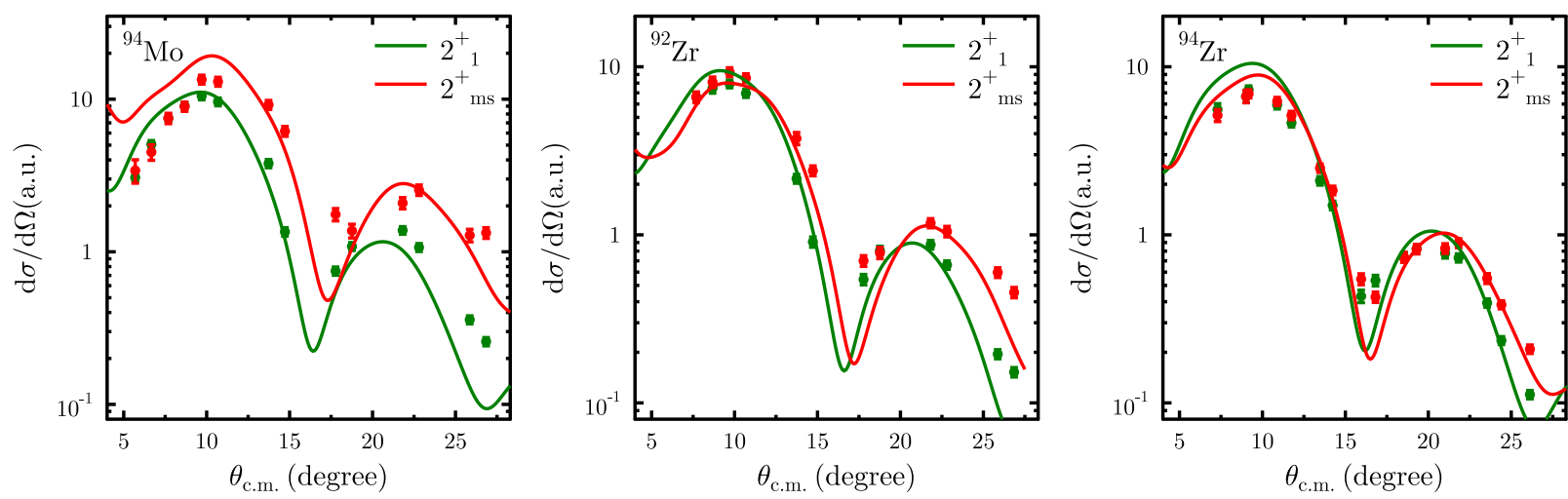

Figure 8.17: Cross sections of the $2_{1}^{+}$and $2_{\mathrm{ms}}^{+}$states in ${ }^{92,94} \mathrm{Zr}$ and ${ }^{94} \mathrm{Mo}$. The cross sections of the mixed-symmetry states are scaled to the one of the corresponding $2_{1}^{+}$states in order to allow a comparison of their shapes.

Figure 8.17 compares the cross sections of the $2_{1}^{+}$states (solid green lines) to the cross sections of the mixed-symmetry states (solid red lines). Clearly, the features of the cross sections of the mixed-symmetry states are shifted to larger scattering angles with respect to the one of the $2_{1}^{+}$ states. The strength of the shift differs for the three nuclei considered. Is it possible to relate this shift to the mixed-symmetric character i.e. to the minus sign of the main neutron component and can we understand the increase of the strength of the shift from ${ }^{94} \mathrm{Zr}$ to ${ }^{94} \mathrm{Mo}$ ?
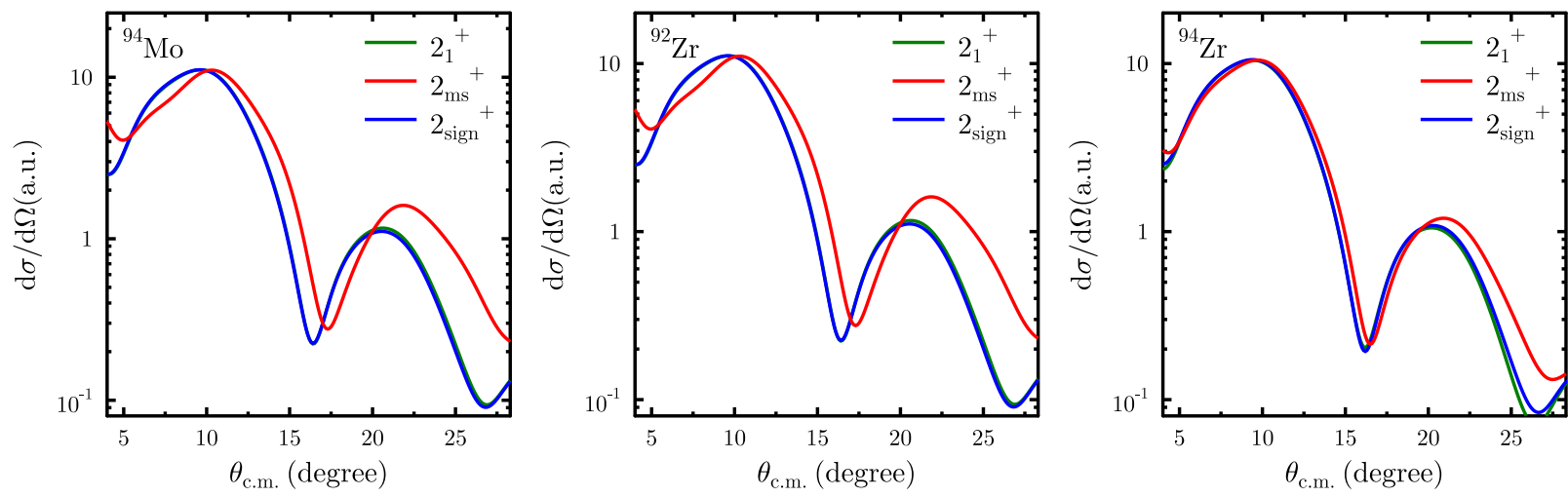

Figure 8.18: Illustration of the dependence of the cross sections of the mixed-symmetry states on the sign of the $\left(2 d_{5 / 2} \otimes 2 d_{5 / 2}\right)_{n}$-2qp-state. Changing its sign artificially from minus (solid red curve) to plus (solid blue curve) shifts the features of the cross sections of the $2_{\mathrm{ms}}^{+}$states to the cross section of the corresponding $2_{1}^{+}$state (solid green curve). The blue and green curves are almost indistinguishable. 
In order to answer this question the minus sign of the $\left(2 d_{5 / 2} \otimes 2 d_{5 / 2}\right)_{n}$-2p-state in the wave functions of the mixed-symmetry states was changed artificially to plus. Although the resulting wave functions are not normalized it is a useful method to investigate the angular dependence of the cross sections on this sign. The corresponding cross sections are shown in Fig. 8.18 (solid blue curves), together with the cross sections of the $2_{1}^{+}$(solid green curves) and $2_{\mathrm{ms}}^{+}$states (solid red curves). The cross sections are scaled to each other in order to allow a comparison of their shapes. In the framework of the theoretical calculation the minus sign is indeed the reason for the observed shift.

Further insights can be obtained from the analysis of the transition radii given for E2transitions through

$$
R_{\mathrm{tr}}=\sqrt{\frac{\int \rho_{\mathrm{tr}} \cdot r^{4} \cdot d r}{\int \rho_{\mathrm{tr}} \cdot r^{2} \cdot d r}}
$$

Here, $\rho_{\text {tr }}$ is the transition density. The calculated proton, neutron and matter transition radii are displayed in Tab. 8.13. The matter transition density is defined as the sum of the proton and neutron transition densities. The $2_{\mathrm{ms}}^{+}$states have slightly smaller proton transition radii than the $2_{1}^{+}$states. A larger difference can be found for the neutron transition radii which differ by 0.15 fm for ${ }^{94} \mathrm{Mo}$ and by $0.06 \mathrm{fm}$ for ${ }^{92} \mathrm{Zr}$. Later it will be shown that this reduction is mainly due to the out-of-phase coupling of the $\left(2 d_{5 / 2} \otimes 2 d_{5 / 2}\right)_{n}$-component to the other 2 qp-states i.e. a direct consequence of the mixed-symmetric character. In contrast, the neutron transition radius of the mixed-symmetry state of ${ }^{94} \mathrm{Zr}$ is larger by $0.06 \mathrm{fm}$ than the one of the $2_{1}^{+}$state. This is partly due to the contribution of the $\left(2 d_{5 / 2} \otimes 3 s_{1 / 2}\right)_{n}$-2qp-state with an amplitude of $37 \%$.

Proton scattering is sensitive to the target protons as well as to the target neutrons, i.e., a more suitable quantity to study the observed shift of the cross sections are the matter transition radii. The differences of the matter transition radii of the $2_{1}^{+}$and $2_{\mathrm{ms}}^{+}$states $\Delta R_{\text {matter }}=R_{\text {matter }}\left(2_{1}^{+}\right)$$R_{\text {matter }}\left(2_{\mathrm{ms}}^{+}\right)$increases from ${ }^{94} \mathrm{Zr}$ to ${ }^{94} \mathrm{Mo}$. In general, an increase of the matter transition radius results in a shift of the cross section to smaller scattering angles. Consequently, the changes of the strength of the shift seen in the experimental and theoretical cross sections are in agreement with the changes of $\Delta R_{\text {matter }}$.

\begin{tabular}{c|cc|cc|cc|c}
\hline \hline & \multicolumn{2}{|c|}{$R_{\mathrm{p}}(\mathrm{fm})$} & \multicolumn{2}{|c|}{$R_{\mathrm{n}}(\mathrm{fm})$} & \multicolumn{2}{|c|}{$R_{\text {matter }}(\mathrm{fm})$} & $\Delta R_{\text {matter }}(\mathrm{fm})$ \\
& $2_{1}^{+}$ & $2_{\mathrm{ms}}^{+}$ & $2_{1}^{+}$ & $2_{\mathrm{ms}}^{+}$ & $2_{1}^{+}$ & $2_{\mathrm{ms}}^{+}$ & \\
\hline${ }^{94} \mathrm{Mo}$ & 5.40 & 5.34 & 6.22 & 6.07 & 5.89 & 5.66 & 0.23 \\
${ }^{92} \mathrm{Zr}$ & 5.40 & 5.37 & 6.24 & 6.18 & 5.92 & 5.80 & 0.12 \\
${ }^{94} \mathrm{Zr}$ & 5.40 & 5.38 & 6.31 & 6.37 & 5.99 & 5.97 & 0.02 \\
\hline \hline
\end{tabular}

Table 8.13: Proton, neutron and matter transition radii of the $2_{1}^{+}$and $2_{\mathrm{ms}}^{+}$states. $\Delta R_{\text {matter }}$ denotes the differences between both matter transition radii of each nucleus. This difference increases from ${ }^{94} \mathrm{Zr}$ to ${ }^{94} \mathrm{Mo}$.

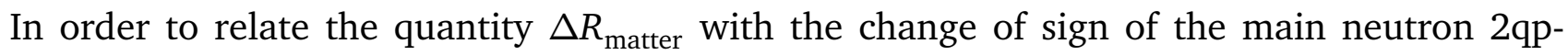
state one has to investigate the theoretical transition densities in detail. Figures 8.19 and 8.20 show the proton and neutron transition densities weighted with $r^{4}$ of ${ }^{94} \mathrm{Mo},{ }^{92} \mathrm{Zr}$ and ${ }^{94} \mathrm{Zr}$. The weighting factor $\mathrm{r}^{4}$ is used to emphasize that proton scattering mainly probes the surface region of the nucleus. Each full density $\rho_{n(p)}$ is decomposed in the contributions of the main neutron or 
proton component - i.e. $\left(2 d_{5 / 2} \otimes 2 d_{5 / 2}\right)_{n}$ or $\left(1 g_{9 / 2} \otimes 1 g_{9 / 2}\right)_{p}$ respectively - and the contributions of all other 2qp-states ( $\rho_{\text {remainder }}$ ). As discussed in Sect. 8.2, the latter transition density is mainly caused by components in the 15-25 MeV energy range which are also responsible for the large $B(E 2)$-values of the $2_{1}^{+}$and $2_{\mathrm{ms}}^{+}$states. The radius of each density is marked with a vertical line.
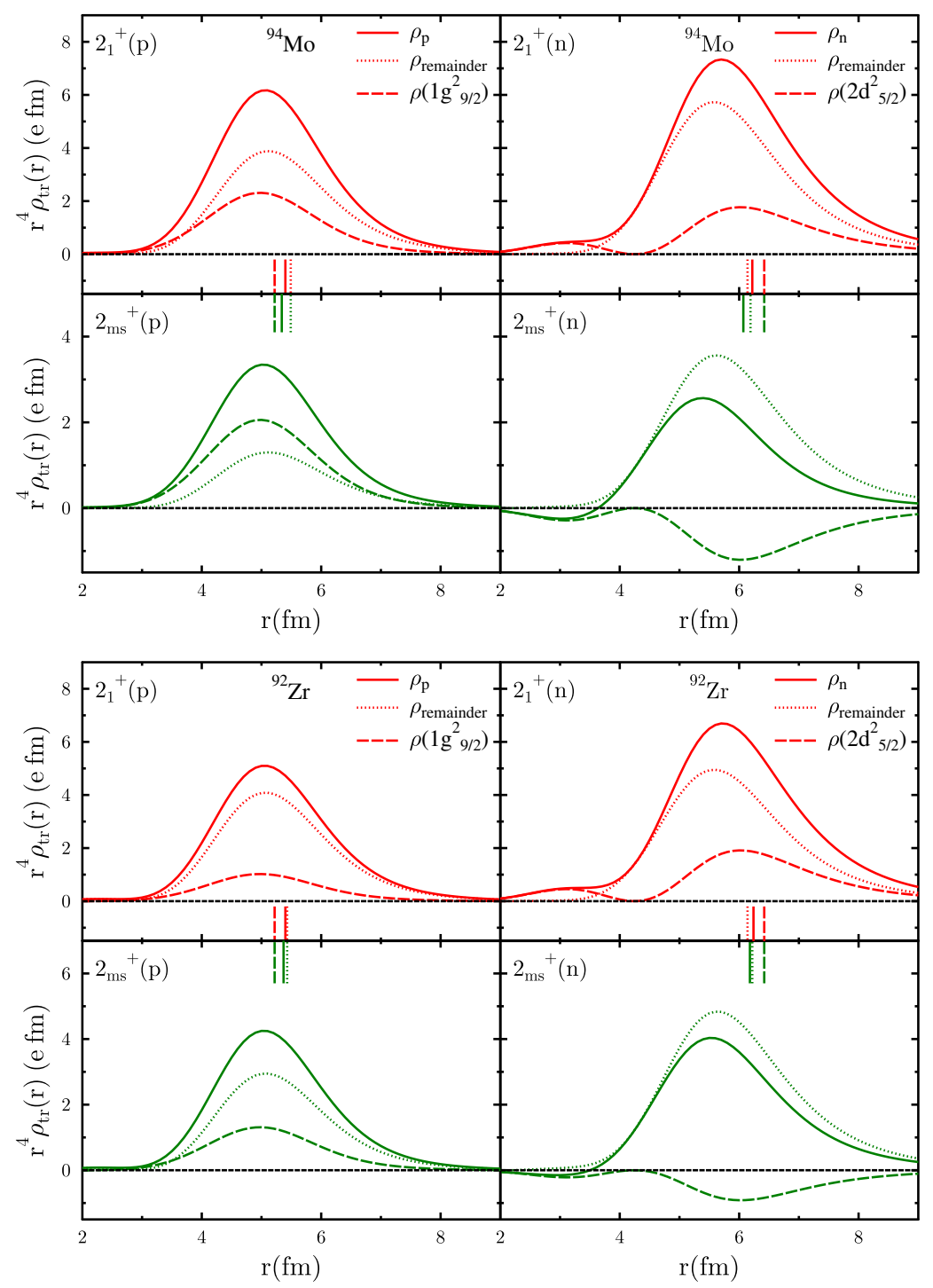

Figure 8.19: Proton and neutron transition densities weighted with $r^{4}$ of ${ }^{94} \mathrm{Mo}$ (left-hand side) and ${ }^{92} \mathrm{Zr}$ (right-hand side). Each transition density is decomposed in the parts stemming from the main components - either the $\left(2 d_{5 / 2} \otimes 2 d_{5 / 2}\right)_{n}$ - or the $\left(1 g_{9 / 2} \otimes 1 g_{9 / 2}\right)_{p^{-}}$ 2qp-state - and all other 2qp-states ( $\left.\rho_{\text {remainder }}\right)$. The out-of-phase coupling of the $\left(2 d_{5 / 2} \otimes 2 d_{5 / 2}\right)_{n}$-component to the remainder part reduces the neutron transition radius of the mixed-symmetry states. The vertical lines mark the transition radii of the corresponding densities.

The transition radii of the 'remainder' parts of the neutron densities of the $2_{1}^{+}$and mixedsymmetry states are similar. The same holds for the proton-remainder densities. In case of the proton densities the $\left(1 g_{9 / 2} \otimes 1 g_{9 / 2}\right)_{p}$-components couple in-phase to the remainder parts in both states. Hence the transition radii of the resulting full densities are similar. On the other hand 


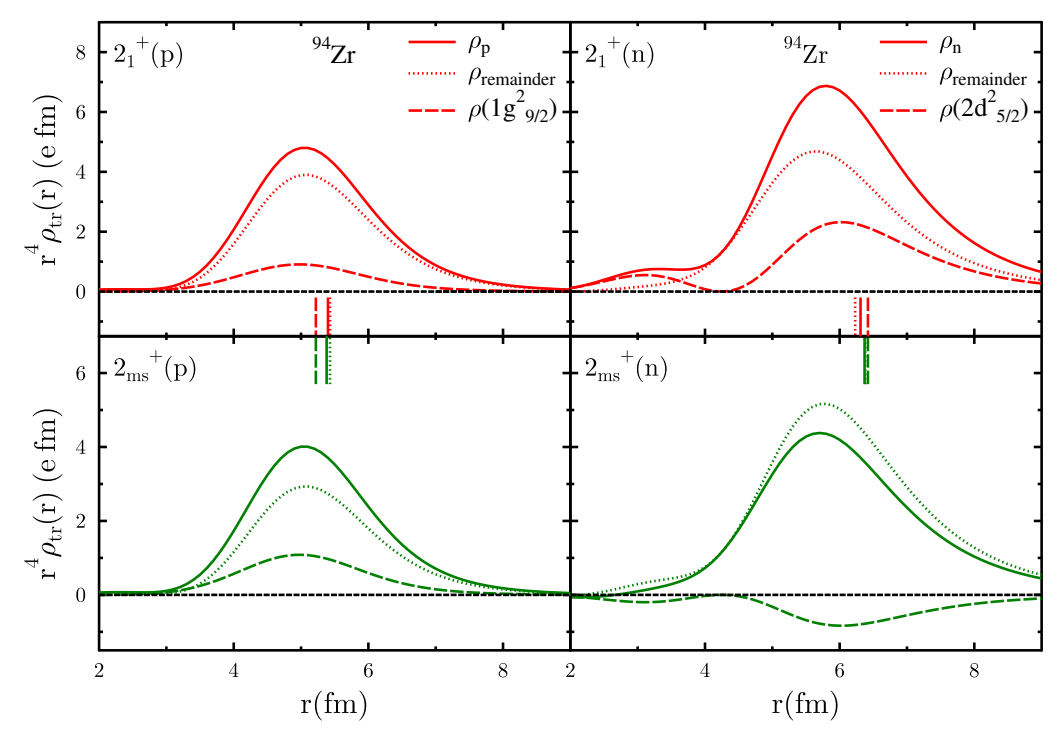

Figure 8.20: Same as Fig. 8.19 for ${ }^{94} \mathrm{Zr}$.

the $\left(2 d_{5 / 2} \otimes 2 d_{5 / 2}\right)_{n}$-component couples in-phase to the neutron-remainder transition densities in case of the $2_{1}^{+}$states and out-of-phase in case of the mixed-symmetry states. Furthermore the radius of the $\left(2 d_{5 / 2} \otimes 2 d_{5 / 2}\right)_{n}$-density is located more in the exterior than the radii of the remainder-parts due to the additional node of the wave function. Hence the radius of the full neutron transition density of the $2_{1}^{+}$state is shifted to the exterior with respect to the remainder density while the one of the mixed-symmetry state is shifted to the interior causing the difference seen in the neutron transition radii.

Moreover one has to keep in mind that the proton transition density of each state is located more in the interior than the neutron transition densities. If one sums up both densities to obtain the matter transition density the resulting matter transition radius depends on the relative amplitudes of both densities. An in-phase coupling of the $\left(2 d_{5 / 2} \otimes 2 d_{5 / 2}\right)_{n}$-component increases the neutron density of the corresponding $2^{+}$state and therefore the matter transition radius. The out-phase coupling in case of the mixed-symmetry state has the opposite effect.

In summary the difference seen in the matter transition radii is caused - in the framework of the QPM - by two reasons. The first one is a change of the neutron transition radii due to the peculiar density of the $\left(2 d_{5 / 2} \otimes 2 d_{5 / 2}\right)_{n}$-2p-state and the opposite sign to the remainder parts. The second reason is also caused by the change of sign of the main neutron component which changes the amplitudes of the full neutron transition density relative to the full proton transition density. Both effects reduce the matter transition radius of the mixed-symmetry state with respect to the one of the $2_{1}^{+}$state. In the QPM the shift of the cross sections seen in experiment can clearly be related to the special character of mixed-symmetry states. The latter effect also explains why the matter transition radius of the $2_{\mathrm{ms}}^{+}$state of ${ }^{94} \mathrm{Zr}$ is smaller than the one of the $2_{1}^{+}$state although its neutron transition radius is larger.

The theoretical analysis provides a simple explanation for the different strengths of the shifts seen in ${ }^{94} \mathrm{Zr}$, ${ }^{92} \mathrm{Zr}$ and ${ }^{94} \mathrm{Mo}$. The shift is strongest in ${ }^{94} \mathrm{Mo}$. In this nucleus the neutron-remainder part relative to the main neutron component is weaker than in ${ }^{92} \mathrm{Zr}$ or ${ }^{94} \mathrm{Zr}$. Hence the discussed change in the neutron transition density is much stronger since the cancellation effect in the exterior is better. ${ }^{92} \mathrm{Zr}$ is an intermediate case between ${ }^{94} \mathrm{Mo}$ and ${ }^{94} \mathrm{Zr}$. In comparison to ${ }^{94} \mathrm{Mo}$ the remainder neutron part is stronger and additionally the contribution of the $\left(2 d_{5 / 2} \otimes 2 d_{5 / 2}\right)_{n^{-}}$ 
component to the wave function of the mixed-symmetry state is reduced from $44.49 \%$ in ${ }^{94} \mathrm{Mo}$ to $28.30 \%$ in ${ }^{92} \mathrm{Zr}$ leading to a weaker shift. In ${ }^{94} \mathrm{Zr}$ this amplitude is further reduced to $20 \%$. Furthermore the remainder neutron part increases. Additionally, the $\left(2 d_{5 / 2} \otimes 3 s_{1 / 2}\right)_{n}$-2qp-state influences the neutron transition density of the mixed-symmetry state of ${ }^{94} \mathrm{Zr}$.

In view of these findings it would be interesting to measure the proton scattering cross section of the mixed-symmetry state of ${ }^{96} \mathrm{Mo}$. Here, the proton $B(E 2)$-value of the mixed-symmetry state is very low $0.08_{-0.01}^{+0.02}$ W.u. [Les07] indicating a small contribution from high-lying 2qpstates. Therefore the shift of the $2_{1}^{+}$and $2_{\mathrm{ms}}^{+}$cross sections might even be stronger in ${ }^{96} \mathrm{Mo}$ because the cancellation effect is stronger. Unfortunately this state was not seen in the proton scattering experiment performed in this thesis. This might be partly due to the described cancellation effect, which not only reduces the neutron transition radius, but also the absolute proton scattering cross sections.

The QPM predicts the changes in the proton transition densities to be small. However it is possible that a part of the observed shift of the proton scattering cross sections is due to different proton transition radii of the $2_{1}^{+}$and $2_{\mathrm{ms}}^{+}$states. Electron scattering at low momentum transfers allows to verify the QPM predictions. As shown in Fig. 8.5 the electron scattering form factors of ${ }^{92} \mathrm{Zr}$ are similar in shape and are well described by the QPM indicating similar proton transition radii. For ${ }^{94} \mathrm{Zr}$ no electron scattering data is available yet. The electron scattering of ${ }^{94}$ Mo shown in Fig. 8.5 is not correctly described by the QPM. Hence it is difficult to draw conclusions about the difference of the proton transition radii.
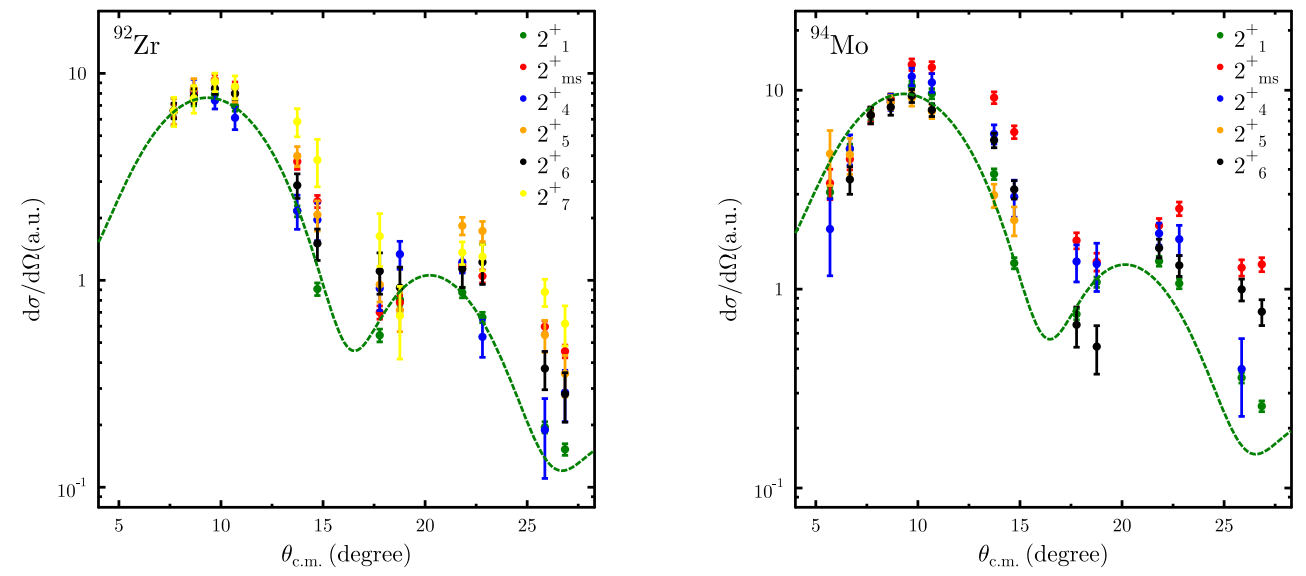

Figure 8.21: Comparison of the cross sections of all measured $2^{+}$states of ${ }^{92} \mathrm{Zr}$ and ${ }^{94} \mathrm{Mo}$. The cross sections are scaled to the one of the corresponding $2_{1}^{+}$state. The dashed green line is a theoretical $2^{+}$cross section calculated with a phenomenological optical potential and the collective model describing the $2_{1}^{+}$states. Two-phonon states are not shown.

The new signature presented in this section proves the change of sign in front of the main neutron 2qp-state of the $2_{1}^{+}$and $2_{\mathrm{ms}}^{+}$states. Therefore it gives clear evidence of the mixed-symmetric character of the $2_{2}^{+}$states of ${ }^{92,94} \mathrm{Zr}$ and the $2_{3}^{+}$state of ${ }^{94} \mathrm{Mo}$ independently of electromagnetic transition strengths. Furthermore the new signature - the relative shift of the features of the proton scattering cross sections of the $2_{1}^{+}$and mixed-symmetry states - provides a sensitive observable to test model wave functions for mixed-symmetry states. A challenge for nuclear structure models is to describe the variation of the strengths of the shifts in ${ }^{92,94} \mathrm{Zr}$ and ${ }^{94} \mathrm{Mo}$ 
correctly. It requires that the model accounts properly for the interplay between high-lying 2qp-states and the main valence components.

Up to this point other $2^{+}$states than the $2_{1}^{+}$and $2_{\mathrm{ms}}^{+}$states were excluded from the discussion. This is justified because all other low-lying $2^{+}$states are mainly non-collective. Therefore the shape of their proton scattering cross sections are determined by their main valence components. The contribution of high-lying 2qp-states, that are responsible for the collectivity of the $2_{1}^{+}$and $2_{\mathrm{ms}}^{+}$states, is small (see e.g. Fig. 8.13). Hence $R_{\text {matter }}$ can be different for each non-collective $2^{+}$state depending on the contributing valence 2qp-states. Figures 8.21 compares the shapes of the cross sections of all measured $2^{+}$states of ${ }^{94} \mathrm{Mo}$ and ${ }^{92} \mathrm{Zr}$. For ${ }^{94} \mathrm{Zr}$ no additional $2^{+}$ states were measured. The cross sections are scaled to each other using the data points at $7.69^{\circ}$. Clearly, the described effect in ${ }^{94} \mathrm{Mo}$ is so strong that the cross section of the mixedsymmetry state is located at larger scattering angles than all other $2^{+}$states. This indicates that the mixed-symmetry state has the smallest matter transition radius. Even in ${ }^{92} \mathrm{Zr}$ - where the effect is weaker - the predominant mixed-symmetry state is the state with the second smallest matter transition radius. In these two nuclei the observed shift is strong enough to allow a differentiation between the mixed-symmetry state and all other $2^{+}$states. In nuclei where the effect is as strong as in ${ }^{92} \mathrm{Zr}$ and ${ }^{94} \mathrm{Mo}$ and no $B(M 1)$-values to the $2_{1}^{+}$state are available, one can identify the mixed-symmetry state by measuring proton scattering cross sections (note that the $2_{7}^{+}$state with the smallest matter transition radius of ${ }^{92} \mathrm{Zr}$ is at an energy of $3488 \mathrm{keV}$ and far away from the expected energy region of the mixed-symmetry state). 


\section{Summary \& outlook}

This work shows that it is indeed possible to detect the two-photon decay in cases where the competing one-photon decay is allowed. Clear evidence was found for the two-photon branch of the decay of the $\frac{11^{-}}{2}$ isomer to the ground state in ${ }^{137} \mathrm{Ba}$. The angular distribution as well as the energy sharing function were measured which in principle allow to draw conclusions about the contributing multipole pairs. The simple model discussed in Sect. 2.2 is not able to account for the experimental data. An analysis in the framework of the Quasi-Particle-Phonon Model (QPM) - or an other nuclear structure model which accounts for the properties of giant resonances - is necessary to understand the measured distributions and to extract a final branching ratio. The branching ratios at angles of $72^{\circ}$ and $144^{\circ}$ - multiplied with the unknown angular correlation function $W^{\prime}(\theta)$ - were measured to be $W^{\prime}\left(72^{\circ}\right) \cdot \Gamma_{\gamma \gamma} / \Gamma_{\gamma}=1.56(23) \cdot 10^{-6}$ and $W^{\prime}\left(144^{\circ}\right) \cdot \Gamma_{\gamma \gamma} / \Gamma_{\gamma}=$ $0.70(18) \cdot 10^{-6}$ respectively (with an energy conditions requiring $\left|E_{1}-E_{2}\right|<300 \mathrm{keV}$ and $\mid E_{1}-$ $E_{2} \mid<250 \mathrm{keV}$ respectively).

This result opens up the opportunity to use the two-photon decay as a general tool to investigate the properties of atomic nuclei from a unique perspective. In contrast to other observables like the energy or the transition strength of an excited state, the two-photon decay probability is an integral quantity, due to the sum over all virtual states which can be connected with the appropriate transition operators to the initial and final states. This quantity might be equivalent to important observables, as for example the diagonal electric dipole polarizability and the diagonal magnetic dipole suceptibility. Hence it can contribute to solve fundamental open problems such as determining the spin quenching factors and restricting the parameters of the equation of state.

The main challenges for a successful two-photon experiment were pointed out. 'Direct' Compton scattering can be blocked with a sufficiently thick lead shield and 'sequential' Compton scattering can be suppressed using appropriate energy and time gates as well as customized lead bricks. One major problem was the background which is present even without a radioactive source. Its origin is not understood. It was possible to suppress this background - using a plastic scintillator veto - by a factor of $\sim 1.5$ which is sufficient to perform this experiment. The main contribution to the final uncertainty of the branching ratio was due to the contribution of the random coincidences.

The experiences gained with this experiment can be used to further improve the experimental setup. A promising alternative to $\mathrm{LaBr}_{3}$-detectors are germanium detectors with an antiCompton-shield. This anti-Compton-shield allows to get rid of a large fraction of the random coincidences, that are mainly caused by photons stemming from the one-photon decay. Furthermore the excellent energy resolution of germanium improves the peak-to-background ratio. On the other hand the time resolution and efficiency of germanium is worse in comparison to $\mathrm{LaBr}_{3}$-detectors. However a first estimate shows that the use of germanium detectors is still advantageous.

In the author's opinion the following measures have to be taken to advance the research on the two-photon decay in atomic nuclei at the Institute of Nuclear Physics in Darmstadt. 
- Initialize a theoretical program to clarify what can be learned from the two-photon decay probability about the structure of atomic nuclei, e.g., is this a useful quantity to complement the diagonal electric dipole polarizability and diagonal magnetic dipole suceptibility? This has to be investigated in a nuclear structure model which is able to calculate the properties of giant resonances as well as the properties of low-lying states.

- One has to find a way to improve the experimental setup. At the Institute of Nuclear Physics in Darmstadt two large germanium detectors with an anti-Compton-shield and the DAGATA detector system [Ali12] are available. It is promising to measure again ${ }^{137} \mathrm{Ba}$ using these detectors.

- The two-photon decay is not limited to the ${ }^{137} \mathrm{Cs}$ source. One can systematically investigate the two-photon decay using other radioactive sources, e.g. a ${ }^{54} \mathrm{Mn}$ source with a $2^{+} \rightarrow 0^{+}$-transition in ${ }^{54} \mathrm{Cr}$ with an energy of $835 \mathrm{keV}$. It is even possible to produce a radioactive element using a particle accelerator, take this element to the experimental setup, and investigate the two-photon decay.

- In case of a $2^{+} \rightarrow 0^{+}$-transition, it is helpful to separate E1-E1 and M1-M1 contributions. In principle this can be done with Compton polarimetry using DAGATA. Whether this is feasible, in case of the two-photon decay, has to be investigated. It might also be possible to use parts of the 'crystal ball' [Met83] (which is available at GSI) for this kind of experiment.

- Lastly it might be possible to populate for example the $2_{1}^{+}$state directly with a particle accelerator. This is a difficult experiment because of beam related background. However this would allow to investigate the two-photon decay probability systematically along an isotopic or isotonic chain.

The development of a new $\mathrm{LaBr}_{3}$-detector setup was also done in the framework of this thesis. The 18 large $\mathrm{LaBr}_{3}$-detectors are mounted to suitable Photo-Multipliers and active voltage dividers. A support structure was designed, which allows to use the detector ball at the QCLAM spectrometer and the photon tagger NEPTUN. Furthermore a digital data acquisition system was set up, based on the SIS3316 digitizers from the company Struck. This is a very compact system since suitable algorithms on the FPGA boards determine energy and time information of the event, i.e. no further VME or NIM electronics are required. The performance of the detector ball was extensively tested. The specifications for time and energy resolution and for the efficiency are met and the system is fully operational. Parts of it were already used for a beam time at the Duke university, a commissioning run at the photon tagger NEPTUN, the PRESPEC campaign at GSI and for the two-photon decay experiment presented in this thesis.

In the other part of this thesis the proton scattering data of ${ }^{92,94} \mathrm{Zr},{ }^{94,96} \mathrm{Mo}$ and ${ }^{70} \mathrm{Zn}$ were analyzed. The angular distributions of the cross sections of the states below $\sim 4 \mathrm{MeV}$ were determined. The spins and parity of 18 states of ${ }^{92} \mathrm{Zr}, 10$ states of ${ }^{94} \mathrm{Zr}, 15$ states of ${ }^{94} \mathrm{Mo}, 18$ states of ${ }^{96} \mathrm{Mo}$ and 24 states of ${ }^{70} \mathrm{Zn}$ were assigned - either firm or tentatively - through a comparison to a calculation using the collective model and a phenomenological optical potential.

Furthermore the data was used to test the ability of the QPM to describe the properties of spherical nuclei in the $N=50, Z=40$ region. The QPM achieves a good description of a large number of observables of quadrupole states including proton scattering cross sections, electron scattering form factors, transition strengths, g-factors and energies. However the QPM has serious problems to account for the properties of $3^{-}$and $4^{+}$states. Clearly, for those states the QPM results are not reliable and improvements on the model itself are necessary. 
Nevertheless some conclusions can be drawn about the possible existence of one-phonon octupole and hexadecapole mixed-symmetry states. The QPM predicts both mixed-symmetry states. The energy of the main fragment of the octupole mixed-symmetry state is predicted to at energies of $>4 \mathrm{MeV}$ due to shell structure effects. If this result is correct, it is experimentally very difficult to investigate this class of states systematically.

The two main components of the hexadecapole mixed-symmetric phonon are much weaker admixed than in case of the quadrupole states. This can be understood in a simple two-state model and is due to the weaker hexadecapole proton-neutron interaction. Furthermore the important $\left[4_{1}^{+}\right]_{[R P A]^{-}},\left[4_{2}^{+}\right]_{[R P A]^{-}}$and $\left[2_{1}^{+} \otimes 2_{1}^{+}\right]_{[R P A]}$-phonons, that determine the structure of the low-lying $4^{+}$states, strongly mix. Since the diagonal $M 1$-transition - which is closely related to the $g$-factors of the sub-components - is large, a $M 1$-transition is no longer a unique signature to identify a mixed-symmetry state in the case of hexadecapole states.

The origin of collectivity of $2^{+}, 3^{-}$and $4^{+}$states in spherical nuclei is investigated in the framework of the QPM. The enhancement is due to the admixture of high-lying two-quasiparticle states (2qp-states) with large transition matrix elements. These 2qp-states are also responsible for the formation of giant resonances. Their matrix elements are enhanced due to the good spatial overlap of the contributing single particle states and their high $j$-values.

Lastly a new signature is introduced which allows to confirm the mixed-symmetric character of the $2_{3}^{+}$state in ${ }^{94} \mathrm{Mo}$ and the $2_{2}^{+}$states in ${ }^{92,94} \mathrm{Zr}$ independently of electromagnetic transition strengths. It is based on the sensitivity of the matter transition radius on the minus sign of the main neutron 2qp-state in the wave function of the mixed-symmetry states. This observable can contribute to deepen our understanding of mixed-symmetry states. 


\section{A Racah algebra}

The U-coefficient in Eq. 2.2 is related to the $6 \mathrm{j}$-symbol by

$$
U(a b c d, e f)=\hat{e} \hat{f}(-)^{a+b+c+d}\left\{\begin{array}{lll}
a & b & c \\
d & e & f
\end{array}\right\}
$$

with $\hat{x}=\sqrt{2 x+1}$. 


\section{B Results of the proton scattering experiments}

This chapter contains the energy, spin and parity assignments and differential proton scattering cross sections of all states where a tentative or firm spin assignment was possible. All cross sections are in $\mathrm{mb} / \mathrm{sr}$.

\section{B.1 Results of the ${ }^{94} \mathrm{Zr}\left(p, p^{\prime}\right)$ experiment}

\begin{tabular}{c|c|cccccccc}
\hline \hline $\mathrm{E}_{\mathrm{x}}(\mathrm{keV})$ & $\mathrm{J}^{\pi}$ & $7.29^{\circ}$ & $9.01^{\circ}$ & $9.17^{\circ}$ & $10.89^{\circ}$ & $11.75^{\circ}$ & $13.47^{\circ}$ & $14.23^{\circ}$ & $15.95^{\circ}$ \\
\hline \hline 0 & $0^{+}$ & $3599(289)$ & $1184(95)$ & $1596(82)$ & $310(16)$ & $76.8(42)$ & $36.3(20)$ & $66.5(34)$ & $104.9(54)$ \\
$909(20)$ & $2^{+}$ & $5.57(46)$ & $6.66(54)$ & $7.30(40)$ & $5.93(32)$ & $4.64(26)$ & $2.10(12)$ & $1.50(9)$ & $0.43(4)$ \\
$1466(10)$ & $4^{+}$ & $0.028(11)$ & $0.066(13)$ & $0.059(15)$ & $0.107(15)$ & $0.216(19)$ & $0.307(21)$ & $0.334(23)$ & $0.358(25)$ \\
$1668(10)$ & $2^{+}$ & $2.42(20)$ & $3.13(26)$ & $3.26(18)$ & $2.93(16)$ & $2.42(14)$ & $1.175(68)$ & $0.866(51)$ & $0.257(19)$ \\
$2059(10)$ & $3^{-}$ & $3.40(28)$ & $7.7(6)$ & $7.72(41)$ & $12.05(63)$ & $14.81(82)$ & $14.48(81)$ & $14.54(76)$ & $9.36(49)$ \\
2330 & $4^{+}$ & $0.016(15)$ & $0.067(20)$ & $0.069(19)$ & $0.171(23)$ & $0.182(35)$ & $0.254(43)$ & $0.293(29)$ & $0.256(22)$ \\
2366 & $\left.2^{+}\right)$ & $0.152(22)$ & $0.161(25)$ & $0.129(21)$ & $0.082(19)$ & $0.109(32)$ & $0.087(35)$ & $0.049(18)$ & $0.052(14)$ \\
$2615(10)$ & $5^{-}$ & $0.0158(10)$ & $0.0092(79)$ & $0.024(15)$ & $0.025(18)$ & $0.0407(81)$ & $0.0995(99)$ & $0.149(15)$ & $0.206(17)$ \\
$3154(10)$ & $4^{+}$ & $0.081(16)$ & $0.115(18)$ & $0.130(26)$ & $0.237(47)$ & $0.256(28)$ & $0.366(30)$ & $0.393(27)$ & $0.404(29)$ \\
$3232(10)$ & $3^{-}$ & $0.211(25)$ & $0.498(51)$ & $0.513(48)$ & $0.702(68)$ & $0.867(58)$ & $0.835(54)$ & $0.751(46)$ & $0.523(36)$
\end{tabular}

Table B.1: Cross sections of the ${ }^{94} \mathrm{Zr}\left(\mathrm{p}, \mathrm{p}^{\prime}\right)$ experiment $\left(7.29^{\circ}-15.95^{\circ}\right)$.

\begin{tabular}{c|c|cccccccc}
\hline \hline $\mathrm{E}_{\mathrm{X}}(\mathrm{keV})$ & $\mathrm{J}^{\pi}$ & $16.81^{\circ}$ & $18.53^{\circ}$ & $19.29^{\circ}$ & $21.01^{\circ}$ & $21.82^{\circ}$ & $23.54^{\circ}$ & $24.40^{\circ}$ & $26.12^{\circ}$ \\
\hline \hline 0 & $0^{+}$ & $107.1(69)$ & $64.8(42)$ & $46.4(28)$ & $17.2(10)$ & $11.52(61)$ & $5.96(32)$ & $6.45(33)$ & $6.49(33)$ \\
$909(20)$ & $2^{+}$ & $0.535(39)$ & $0.736(48)$ & $0.826(51)$ & $0.777(50)$ & $0.730(41)$ & $0.393(22)$ & $0.235(13)$ & $0.112(6)$ \\
$1466(10)$ & $4^{+}$ & $0.317(21)$ & $0.200(1)$ & $0.149(11)$ & $0.110(9)$ & $0.0686(63)$ & $0.0387(34)$ & $0.0323(23)$ & $0.0315(23)$ \\
$1668(10)$ & $2^{+}$ & $0.201(14)$ & $0.347(24)$ & $0.388(25)$ & $0.391(26)$ & $0.420(26)$ & $0.259(18)$ & $0.18(1)$ & $0.0983(57)$ \\
$2059(10)$ & $3^{-}$ & $6.31(41)$ & $2.92(19)$ & $1.98(12)$ & $1.337(81)$ & $1.439(78)$ & $1.605(87)$ & $1.624(85)$ & $1.396(73)$ \\
2330 & $4^{+}$ & $0.204(17)$ & $0.121(11)$ & $0.0997(89)$ & $0.062(6)$ & $0.0689(64)$ & $0.0409(54)$ & $0.0463(45)$ & $0.039(4)$ \\
2366 & $\left.2^{+}\right)$ & $0.075(10)$ & $0.0666(79)$ & $0.0304(57)$ & $0.0158(42)$ & $0.0067(41)$ & $0.0127(42)$ & $0.0133(33)$ & $0.0270(34)$ \\
$2615(10)$ & $5^{-}$ & $0.268(19)$ & $0.343(23)$ & $0.341(22)$ & $0.360(23)$ & $0.357(21)$ & $0.310(19)$ & $0.255(14)$ & $0.178(10)$ \\
$3154(10)$ & $4^{+}$ & $0.374(25)$ & $0.3096(22)$ & $0.2419(16)$ & $0.155(11)$ & $0.1305(91)$ & $0.0924(75)$ & $0.0746(49)$ & $0.0599(44)$ \\
$3232(10)$ & $3^{-}$ & $0.374(25)$ & $0.226(16)$ & $0.208(14)$ & $0.163(12)$ & $0.165(11)$ & $0.1334(96)$ & $0.106(7)$ & $0.0829(57)$
\end{tabular}

Table B.2: Cross sections of the ${ }^{94} \operatorname{Zr}\left(p, p^{\prime}\right)$ experiment $\left(16.81^{\circ}-26.12^{\circ}\right)$. 
B.2 Results of the ${ }^{92} \mathrm{Zr}\left(\mathbf{p}, \mathbf{p}^{\prime}\right)$ experiment

\begin{tabular}{c|c|cccccc}
\hline \hline $\mathrm{E}_{\mathrm{x}}(\mathrm{keV})$ & $\mathrm{J}^{\pi}$ & $7.69^{\circ}$ & $8.67^{\circ}$ & $9.70^{\circ}$ & $10.69^{\circ}$ & $13.74^{\circ}$ & $14.72^{\circ}$ \\
\hline \hline 0 & $0^{+}$ & $3214(213)$ & $1834(123)$ & $854(47)$ & $329(19)$ & $41.2(23)$ & $77.9(43)$ \\
$934(10)$ & $2^{+}$ & $6.60(44)$ & $7.46(50)$ & $7.92(43)$ & $6.95(38)$ & $2.16(13)$ & $0.908(64)$ \\
$1490(10)$ & $4^{+}$ & $0.075(21)$ & $0.039(15)$ & $0.132(20)$ & $0.198(21)$ & $0.410(44)$ & $0.471(32)$ \\
$1848(10)$ & $2^{+}$ & $1.42(11)$ & $1.74(13)$ & $2.00(12)$ & $1.85(11)$ & $0.806(67)$ & $0.518(35)$ \\
$2059(10)$ & $2^{+}$ & $0.115(21)$ & $0.093(19)$ & $0.136(21)$ & $0.133(17)$ & $0.178(28)$ & $0.164(16)$ \\
$2340(10)$ & $3^{-}$ & $2.37(17)$ & $3.93(27)$ & $6.15(34)$ & $7.64(41)$ & $9.85(55)$ & $8.15(43)$ \\
$2393(10)$ & $4^{+}$ & $0.058(24)$ & $0.151(38)$ & $0.125(32)$ & $0.262(37)$ & $0.296(64)$ & $0.343(41)$ \\
$2488(10)$ & $5^{-}$ & - & - & - & $0.026(10)$ & $0.167(27)$ & $0.212(18)$ \\
$2817(10)$ & $2^{+}$ & $0.190(28)$ & $0.239(30)$ & $0.212(19)$ & $0.175(22)$ & $0.062(12)$ & $0.056(12)$ \\
$2862(10)$ & $4^{+}$ & - & $0.050(16)$ & $0.079(13)$ & $0.115(19)$ & $0.285(23)$ & $0.275(22)$ \\
$2950(10)$ & $6^{+}$ & - & - & - & - & $0.0068(65)$ & $0.0075(56)$ \\
$3050(10)$ & $2^{+}$ & $0.175(25)$ & $0.222(28)$ & $0.245(20)$ & $0.211(18)$ & $0.106(12)$ & $0.0550(91)$ \\
$3183(10)$ & $\left.4^{+}\right)$ & - & - & $0.0324(76)$ & $0.0554(92)$ & $0.168(16)$ & $0.201(19)$ \\
$3238(20)$ & $\left(4^{+}, 5^{-}\right)$ & - & - & - & - & $0.059(34)$ & $0.092(25)$ \\
$3262(20)$ & $2^{+}$ & $0.667(57)$ & $0.786(64)$ & $0.853(52)$ & $0.809(50)$ & $0.291(39)$ & $0.152(26)$ \\
$3340(20)$ & $5^{-}$ & - & - & $0.064(10)$ & $0.085(11)$ & $0.094(15)$ & $0.138(17)$ \\
$3446(20)$ & $3^{-}$ & $0.206(29)$ & $0.350(38)$ & $0.540(38)$ & $0.721(49)$ & $1.058(65)$ & $0.938(61)$ \\
$3488(20)$ & $2^{+}$ & $0.160(26)$ & $0.184(28)$ & $0.221(23)$ & $0.210(27)$ & $0.142(22)$ & $0.093(24)$
\end{tabular}

Table B.3: Cross sections of the ${ }^{92} \mathrm{Zr}\left(\mathrm{p}, \mathrm{p}^{\prime}\right)$ experiment $\left(7.69^{\circ}-14.72^{\circ}\right)$.

\begin{tabular}{c|c|cccccc}
\hline \hline $\mathrm{E}_{\mathrm{x}}(\mathrm{keV})$ & $\mathrm{J}^{\pi}$ & $17.78^{\circ}$ & $18.76^{\circ}$ & $21.82^{\circ}$ & $22.81^{\circ}$ & $25.86^{\circ}$ & $26.85^{\circ}$ \\
\hline \hline 0 & $0^{+}$ & $90.3(50)$ & $62.0(34)$ & $11.53(64)$ & $6.84(39)$ & $6.26(36)$ & $5.89(37)$ \\
$934(10)$ & $2^{+}$ & $0.543(39)$ & $0.806(49)$ & $0.874(51)$ & $0.662(38)$ & $0.195(12)$ & $0.152(10)$ \\
$1490(10)$ & $4^{+}$ & $0.346(23)$ & $0.231(16)$ & $0.075(6)$ & $0.056(5)$ & $0.037(3)$ & $0.042(4)$ \\
$1848(10)$ & $2^{+}$ & $0.151(11)$ & $0.168(13)$ & $0.253(16)$ & $0.225(16)$ & $0.128(9)$ & $0.098(7)$ \\
$2059(10)$ & $2^{+}$ & $0.0798(7)$ & $0.0571(66)$ & $0.02132(32)$ & $0.0253(35)$ & $0.0349(34)$ & $0.0300(31)$ \\
$2340(10)$ & $3^{-}$ & $3.51(19)$ & $2.12(11)$ & $0.984(54)$ & $1.04(6)$ & $1.03(6)$ & $0.903(50)$ \\
$2393(10)$ & $4^{+}$ & $0.217(16)$ & $0.149(14)$ & $0.0612(62)$ & $0.0527(60)$ & $0.0429(56)$ & $0.0435(49)$ \\
$2488(10)$ & $5^{-}$ & $0.425(26)$ & $0.423(26)$ & $0.461(27)$ & $0.411(25)$ & $0.215(13)$ & $0.189(12)$ \\
$2817(10)$ & $2^{+}$ & $0.0263(57)$ & $0.0384(59)$ & $0.0351(40)$ & $0.0153(31)$ & $0.0054(23)$ & $0.0082(23)$ \\
$2862(10)$ & $4^{+}$ & $0.253(18)$ & $0.183(14)$ & $0.0602(55)$ & $0.0439(48)$ & $0.0236(31)$ & $0.0266(33)$ \\
$2950(10)$ & $6^{+}$ & $0.0551(69)$ & $0.0617(76)$ & $0.0685(58)$ & $0.0698(64)$ & $0.0469(42)$ & $0.0482(42)$ \\
$3050(10)$ & $2^{+}$ & $0.0253(45)$ & $0.0190(41)$ & $0.0487(48)$ & $0.0459(51)$ & $0.0144(26)$ & $0.0094(21)$ \\
$3183(10)$ & $\left.4^{+}\right)$ & $0.281(18)$ & $0.220(15)$ & $0.162(11)$ & $0.1219(91)$ & $0.0532(47)$ & $0.0426(45)$ \\
$3238(20)$ & $\left(4^{+}, 5^{-}\right)$ & $0.132(25)$ & $0.124(24)$ & $0.086(21)$ & $0.033(26)$ & $0.0405(80)$ & $0.0322(73)$ \\
$3262(20)$ & $2^{+}$ & $0.112(25)$ & $0.093(24)$ & $0.115(22)$ & $0.124(27)$ & $0.0379(79)$ & $0.0285(77)$ \\
$3340(20)$ & $5^{-}$ & $0.209(16)$ & $0.192(18)$ & $0.198(14)$ & $0.149(13)$ & $0.1076(85)$ & $0.0834(70)$ \\
$3446(20)$ & $3^{-}$ & $0.474(30)$ & $0.306(20)$ & $0.1272(90)$ & $0.1124(86)$ & $0.1035(75)$ & $0.0915(70)$ \\
$3488(20)$ & $2^{+}$ & $0.040(11)$ & $0.0164(63)$ & $0.0331(41)$ & $0.0316(46)$ & $0.0214(32)$ & $0.015(3)$
\end{tabular}

Table B.4: Cross sections of the ${ }^{92} \mathrm{Zr}\left(\mathrm{p}, \mathrm{p}^{\prime}\right)$ experiment $\left(17.78^{\circ}-26.85^{\circ}\right)$. 


\section{B.3 Results of the ${ }^{94} \mathrm{Mo}\left(p, p^{\prime}\right)$ experiment}

\begin{tabular}{c|c|ccccccc}
\hline \hline$E_{\mathrm{X}}(\mathrm{keV})$ & $\mathrm{J}^{\pi}$ & $5.69^{\circ}$ & $6.67^{\circ}$ & $7.69^{\circ}$ & $8.67^{\circ}$ & $9.70^{\circ}$ & $10.68^{\circ}$ & $13.73^{\circ}$ \\
\hline \hline 0 & $0^{+}$ & $6051(347)$ & $4076(237)$ & $2548(147)$ & $1422(82)$ & $825(47)$ & $386(22)$ & $36.9(21)$ \\
$872(10)$ & $2^{+}$ & $3.07(19)$ & $5.06(30)$ & $7.50(44)$ & $9.04(53)$ & $10.5(6)$ & $9.62(56)$ & $3.79(23)$ \\
$1573(10)$ & $4^{+}$ & - & $0.040(13)$ & $0.0411(93)$ & $0.088(11)$ & $0.187(18)$ & $0.274(22)$ & $0.832(53)$ \\
$1865(10)$ & $\left(2^{+}\right)$ & - & - & $0.070(14)$ & $0.067(11)$ & $0.076(15)$ & $0.059(12)$ & $0.0152(62)$ \\
$2066(10)$ & $2^{+}$ & $0.191(33)$ & $0.251(29)$ & $0.419(33)$ & $0.500(35)$ & $0.75(5)$ & $0.728(49)$ & $0.513(35)$ \\
$2293(10)$ & $4^{+}$ & - & - & - & - & - & $0.029(18)$ & $0.101(13)$ \\
$2391(10)$ & $2^{+}$ & $0.048(20)$ & $0.122(21)$ & $0.179(17)$ & $0.209(19)$ & $0.280(28)$ & $0.262(28)$ & $0.144(16)$ \\
2423 & $6^{+}$ & - & - & - & - & - & - & - \\
$2534(10)$ & $3^{-}$ & $0.381(38)$ & $0.777(57)$ & $1.623(97)$ & $2.67(16)$ & $4.19(24)$ & $4.97(29)$ & $7.88(44)$ \\
$2606(10)$ & $\left.5^{-}\right)$ & - & - & - & - & - & $0.121(28)$ & $0.188(19)$ \\
$2763(10)$ & $4^{+}$ & - & $0.036(21)$ & $0.012(10)$ & $0.0331(87)$ & $0.041(14)$ & $0.076(11)$ & $0.377(27)$ \\
$2862(10)$ & $2^{+}+6^{+}$ & $0.131(40)$ & $0.130(27)$ & $0.205(20)$ & $0.237(19)$ & $0.250(23)$ & $0.214(17)$ & $0.081(11)$ \\
$2998(10)$ & $3^{-}$ & $0.030(29)$ & $0.075(23)$ & $0.107(15)$ & $0.186(17)$ & $0.358(28)$ & $0.395(27)$ & $0.406(28)$ \\
$3439(20)$ & $\left(4^{+}\right)$ & - & $0.072(18)$ & $0.110(16)$ & $0.137(17)$ & $0.173(17)$ & $0.186(15)$ & $0.347(24)$ \\
$3779(20)$ & $2^{+}$ & - & $0.118(19)$ & $0.249(24)$ & $0.272(24)$ & $0.311(23)$ & $0.264(20)$ & $0.186(16)$
\end{tabular}

Table B.5: Cross sections of the ${ }^{94} \mathrm{Mo}\left(\mathrm{p}, \mathrm{p}^{\prime}\right)$ experiment $\left(5.69^{\circ}-13.73^{\circ}\right)$.

\begin{tabular}{c|c|ccccccc}
\hline \hline$E_{\mathrm{X}}(\mathrm{keV})$ & $\mathrm{J}^{\pi}$ & $14.72^{\circ}$ & $17.77^{\circ}$ & $18.76^{\circ}$ & $21.81^{\circ}$ & $22.80^{\circ}$ & $25.86^{\circ}$ & $26.84^{\circ}$ \\
\hline \hline 0 & $0^{+}$ & $69.3(40)$ & $78.5(46)$ & $55.6(33)$ & $10.36(60)$ & $6.09(36)$ & $5.71(33)$ & $5.29(31)$ \\
$872(10)$ & $2^{+}$ & $1.352(87)$ & $0.749(46)$ & $1.085(65)$ & $1.383(79)$ & $1.068(64)$ & $0.359(22)$ & $0.258(16)$ \\
$1573(10)$ & $4^{+}$ & $0.911(57)$ & $0.602(42)$ & $0.600(46)$ & $0.284(25)$ & $0.1159(93)$ & $0.0956(71)$ & $0.0779(61)$ \\
$1865(10)$ & $\left(2^{+}\right)$ & $0.0213(63)$ & $0.0402(54)$ & $0.0286(48)$ & - & - & $0.0167(28)$ & $0.0128(20)$ \\
$2066(10)$ & $2^{+}$ & $0.344(25)$ & $0.098(9)$ & $0.0767(79)$ & $0.1166(99)$ & $0.142(11)$ & $0.0716(68)$ & $0.0744(60)$ \\
$2293(10)$ & $4^{+}$ & $0.088(14)$ & $0.104(10)$ & $0.099(11)$ & $0.0330(72)$ & $0.0148(53)$ & - & - \\
$2391(10)$ & $2^{+}$ & $0.070(15)$ & $0.0329(69)$ & $0.0320(87)$ & $0.0455(58)$ & $0.0426(74)$ & $0.0095(40)$ & - \\
2423 & $6^{+}$ & $0.017(11)$ & $0.0457(74)$ & $0.0560(97)$ & $0.0742(72)$ & $0.076(10)$ & $0.0495(65)$ & - \\
$2534(10)$ & $3^{-}$ & $6.63(37)$ & $2.72(15)$ & $1.627(96)$ & $0.789(47)$ & $0.732(45)$ & $0.804(46)$ & $0.666(39)$ \\
$2606(10)$ & $\left(5^{-}\right)$ & $0.211(21)$ & $0.407(27)$ & $0.37(3)$ & $0.451(29)$ & $0.364(25)$ & $0.264(17)$ & $0.178(12)$ \\
$2763(10)$ & $4^{+}$ & $0.421(31)$ & $0.416(26)$ & $0.371(24)$ & $0.194(14)$ & $0.1125(91)$ & $0.0551(52)$ & $0.0449(47)$ \\
$2862(10)$ & $2^{+}+6^{+}$ & $0.061(10)$ & $0.0454(55)$ & $0.0502(59)$ & $0.0643(67)$ & $0.0579(59)$ & $0.0355(40)$ & $0.0365(41)$ \\
$2998(10)$ & $3^{-}$ & $0.310(24)$ & $0.0753(69)$ & $0.0502(53)$ & $0.0710(69)$ & $0.0792(70)$ & $0.0723(79)$ & $0.0396(41)$ \\
$3439(20)$ & $\left(4^{+}\right)$ & $0.299(21)$ & $0.230(17)$ & $0.173(13)$ & $0.0898(77)$ & $0.0592(57)$ & $0.0467(55)$ & - \\
$3779(20)$ & $2^{+}$ & $0.105(11)$ & $0.0219(50)$ & $0.0170(47)$ & $0.0533(57)$ & $0.0436(53)$ & $0.0331(42)$ & $0.0255(38)$
\end{tabular}

Table B.6: Cross sections of the ${ }^{94} \mathrm{Mo}\left(\mathrm{p}, \mathrm{p}^{\prime}\right)$ experiment $\left(14.72^{\circ}-26.84^{\circ}\right)$. 
B.4 Results of the ${ }^{96} \mathrm{Mo}\left(p, p^{\prime}\right)$ experiment

\begin{tabular}{c|c|ccccccc}
\hline \hline$E_{\mathrm{X}}(\mathrm{keV})$ & $\mathrm{J}^{\pi}$ & $7.19^{\circ}$ & $8.91^{\circ}$ & $9.22^{\circ}$ & $10.94^{\circ}$ & $11.75^{\circ}$ & $13.47^{\circ}$ & $14.28^{\circ}$ \\
\hline \hline 0 & $0^{+}$ & $4726(334)$ & $1735(121)$ & $1231(99)$ & $219(18)$ & $90.4(90)$ & $32.9(32)$ & $73.4(43)$ \\
$773(20)$ & $2^{+}$ & $14.9(10)$ & $20.3(14)$ & $20.0(16)$ & $16.4(13)$ & $14.1(13)$ & $6.54(61)$ & $3.83(21)$ \\
$1498(10)$ & $2^{+}$ & $0.533(42)$ & $0.825(61)$ & $0.878(69)$ & $0.936(74)$ & $0.872(80)$ & $0.537(52)$ & $0.415(27)$ \\
$1628(10)$ & $4^{+}$ & $0.069(12)$ & $0.136(14)$ & $0.190(17)$ & $0.326(28)$ & $0.408(38)$ & $0.550(53)$ & $0.612(38)$ \\
$1870(10)$ & $4^{+}$ & $0.0306(85)$ & $0.086(11)$ & $0.101(11)$ & $0.227(21)$ & $0.272(30)$ & $0.355(37)$ & $0.423(28)$ \\
$2235(10)$ & $3^{-}$ & $2.41(17)$ & $6.19(42)$ & $6.86(52)$ & $10.95(84)$ & $11.78(106)$ & $11.62(104)$ & $10.92(58)$ \\
$2481(10)$ & $4^{+}$ & $0.0329(80)$ & $0.081(10)$ & $0.096(11)$ & $0.252(24)$ & $0.311(33)$ & $0.469(47)$ & $0.581(37)$ \\
$2539(10)$ & $\left.2^{+}\right)$ & $0.202(18)$ & $0.238(21)$ & $0.196(18)$ & $0.136(15)$ & $0.102(15)$ & $0.0403(86)$ & $0.0421(79)$ \\
$2626(10)$ & $4^{+}$ & $0.0153(71)$ & $0.0553(85)$ & $0.0669(89)$ & $0.147(16)$ & $0.189(22)$ & $0.285(31)$ & $0.336(23)$ \\
$2700(10)$ & $\left.2^{+}\right)$ & $0.0365(86)$ & $0.101(12)$ & $0.096(11)$ & $0.105(14)$ & $0.111(16)$ & $0.081(13)$ & $0.0626(92)$ \\
$2739(10)$ & $6^{+}$ & - & $0.0112(71)$ & $0.0112(67)$ & $0.030(10)$ & $0.032(11)$ & $0.065(12)$ & $0.087(11)$ \\
$3018(20)$ & $5^{-}$ & - & $0.0388(98)$ & $0.0306(71)$ & $0.0709(98)$ & $0.137(16)$ & $0.256(26)$ & $0.379(25)$ \\
$3137(20)$ & $2^{+}$ & $0.216(21)$ & $0.348(29)$ & $0.363(30)$ & $0.332(27)$ & $0.285(28)$ & $0.143(15)$ & $0.0924(90)$ \\
$3179(20)$ & $3^{-}$ & $0.144(17)$ & $0.315(26)$ & $0.352(29)$ & $0.513(41)$ & $0.568(53)$ & $0.526(49)$ & $0.480(28)$ \\
$3233(20)$ & $4^{+}$ & $0.019(10)$ & $0.0435(87)$ & $0.0474(64)$ & $0.0944(98)$ & $0.102(12)$ & $0.145(15)$ & $0.145(11)$ \\
$3349(20)$ & $2^{+}$ & $0.182(18)$ & $0.260(22)$ & $0.258(22)$ & $0.239(20)$ & $0.210(21)$ & $0.110(12)$ & $0.0646(65)$ \\
$3428(20)$ & $4^{+}, 5^{-}$ & $0.0182(77)$ & $0.059(10)$ & $0.0460(66)$ & $0.118(13)$ & $0.156(18)$ & $0.249(26)$ & $0.308(19)$ \\
$3601(20)$ & $\left(3^{-}\right)$ & $0.106(13)$ & $0.142(25)$ & $0.126(14)$ & $0.100(17)$ & $0.078(14)$ & $0.043(17)$ & $0.0802(80)$
\end{tabular}

Table B.7: Cross sections of the ${ }^{96} \mathrm{Mo}\left(\mathrm{p}, \mathrm{p}^{\prime}\right)$ experiment $\left(7.19^{\circ}-14.28^{\circ}\right)$.

\begin{tabular}{c|c|ccccccc}
\hline \hline$E_{\mathrm{X}}(\mathrm{keV})$ & $\mathrm{J}^{\pi}$ & $16.00^{\circ}$ & $16.81^{\circ}$ & $18.53^{\circ}$ & $19.34^{\circ}$ & $21.06^{\circ}$ & $21.87^{\circ}$ & $23.59^{\circ}$ \\
\hline \hline 0 & $0^{+}$ & $109.4(62)$ & $113.5(75)$ & $68.8(47)$ & $49.6(47)$ & $17.1(16)$ & $9.79(59)$ & $5.95(35)$ \\
$773(20)$ & $2^{+}$ & $1.152(76)$ & $1.137(78)$ & $1.89(0.13)$ & $2.49(23)$ & $2.42(23)$ & $2.20(13)$ & $1.296(78)$ \\
$1498(10)$ & $2^{+}$ & $0.152(11)$ & $0.0904(78)$ & $0.0945(85)$ & $0.0944(94)$ & $0.132(14)$ & $0.1433(98)$ & $0.1261(84)$ \\
$1628(10)$ & $4^{+}$ & $0.577(34)$ & $0.536(36)$ & $0.348(24)$ & $0.282(27)$ & $0.161(16)$ & $0.132(11)$ & $0.0946(66)$ \\
$1870(10)$ & $4^{+}$ & $0.411(27)$ & $0.390(29)$ & $0.279(22)$ & $0.227(22)$ & $0.115(12)$ & $0.0760(61)$ & $0.0491(46)$ \\
$2235(10)$ & $3^{-}$ & $7.20(39)$ & $5.61(36)$ & $2.54(17)$ & $1.70(16)$ & $1.07(10)$ & $1.197(70)$ & $1.338(77)$ \\
$2481(10)$ & $4^{+}$ & $0.605(37)$ & $0.653(46)$ & $0.511(37)$ & $0.474(45)$ & $0.293(30)$ & $0.239(17)$ & $0.127(11)$ \\
$2539(10)$ & $\left.2^{+}\right)$ & $0.0601(83)$ & $0.0678(87)$ & $0.0749(90)$ & $0.0663(82)$ & $0.0322(60)$ & $0.0307(59)$ & $0.0192(46)$ \\
$2626(10)$ & $4^{+}$ & $0.320(22)$ & $0.335(26)$ & $0.230(18)$ & $0.187(19)$ & $0.092(10)$ & $0.0608(72)$ & $0.0440(59)$ \\
$2700(10)$ & $\left.2^{+}\right)$ & $0.0263(70)$ & $0.0215(65)$ & $0.0249(70)$ & $0.0204(59)$ & $0.0369(74)$ & $0.0226(77)$ & $0.0207(72)$ \\
$2739(10)$ & $6^{+}$ & $0.161(14)$ & $0.198(17)$ & $0.264(21)$ & $0.320(31)$ & $0.332(33)$ & $0.336(23)$ & $0.330(22)$ \\
$3018(20)$ & $5^{-}$ & $0.552(32)$ & $0.634(43)$ & $0.669(46)$ & $0.683(64)$ & $0.540(51)$ & $0.438(28)$ & $0.319(19)$ \\
$3137(20)$ & $2^{+}$ & $0.0279(64)$ & $0.0246(51)$ & $0.0472(79)$ & $0.0653(98)$ & $0.0727(89)$ & $0.0616(91)$ & $0.0473(53)$ \\
$3179(20)$ & $3^{-}$ & $0.282(18)$ & $0.211(16)$ & $0.0709(90)$ & $0.0632(95)$ & $0.0517(71)$ & $0.0558(88)$ & $0.0812(68)$ \\
$3233(20)$ & $4^{+}$ & $0.1240(95)$ & $0.123(10)$ & $0.0689(79)$ & $0.0594(77)$ & $0.0221(37)$ & $0.0205(55)$ & $0.0155(31)$ \\
$3349(20)$ & $2^{+}$ & $0.0188(41)$ & $0.0170(38)$ & $0.0163(43)$ & $0.0322(53)$ & $0.0353(45)$ & $0.0354(57)$ & $0.0202(33)$ \\
$3428(20)$ & $4^{+}, 5^{-}$ & $0.333(20)$ & $0.386(27)$ & $0.306(22)$ & $0.294(29)$ & $0.201(19)$ & $0.147(12)$ & $0.0877(67)$ \\
$3601(20)$ & $3^{-}$ & $0.0458(60)$ & $0.0486(61)$ & $0.0284(59)$ & $0.0178(48)$ & $0.0170(59)$ & $0.0180(46)$ & $0.0067(35)$
\end{tabular}

Table B.8: Cross sections of the ${ }^{96} \mathrm{Mo}\left(p, p^{\prime}\right)$ experiment $\left(16.00^{\circ}-23.59^{\circ}\right)$. 
B.5 Results of the ${ }^{70} \mathrm{Zn}\left(\mathrm{p}, \mathrm{p}^{\prime}\right)$ experiment

\begin{tabular}{|c|c|c|c|c|c|c|c|c|c|}
\hline$\overline{\overline{E_{\mathrm{x}}(\mathrm{keV})}}$ & $\overline{\mathrm{J}^{\pi}}$ & $7.28^{\circ}$ & $9.00^{\circ}$ & $9.36^{\circ}$ & $11.09^{\circ}$ & $11.90^{\circ}$ & $13.63^{\circ}$ & $14.34^{\circ}$ & $16.07^{\circ}$ \\
\hline$\overline{0}$ & $0^{+}$ & 3532(229) & 1525(99) & 1411(122) & $369.8(320)$ & $\begin{array}{l}60.7(83) \\
\end{array}$ & $23.87(123)$ & $25.26(130)$ & 48.44(249) \\
\hline $875(20)$ & $2^{+}$ & 13.84(90) & $19.45(126)$ & 22.30 (193) & $19.37(168)$ & 18.93(97) & $11.19(58)$ & 9.51(49) & $3.60(19)$ \\
\hline 1761(10) & $2^{+}$ & $0.298(22)$ & $0.560(38)$ & $0.685(62)$ & $0.740(72)$ & $0.830(51)$ & $0.600(36)$ & $0.559(33)$ & $0.353(21)$ \\
\hline 1787 & $4^{+}$ & & & & $0.032(24)$ & $0.136(22)$ & $0.293(22)$ & $0.298(20)$ & $0.341(21)$ \\
\hline 1958(10) & $2^{+}$ & $0.282(21)$ & $0.412(29)$ & $0.471(44)$ & $0.477(45)$ & $0.496(30)$ & $0.363(22)$ & $0.312(19)$ & $0.162(10)$ \\
\hline $2667(10)$ & $2^{+}$ & $0.0211(60)$ & $0.0425(62)$ & $0.029(10)$ & $0.039(10)$ & $0.0367(76)$ & $0.0177(60)$ & $0.0239(99)$ & $0.0125(60)$ \\
\hline 2695(10) & $4^{+}$ & & - & $0.021(13)$ & $0.031(13)$ & $0.071(11)$ & $0.113(12)$ & $0.099(15)$ & $0.128(13)$ \\
\hline $2865(10)$ & $3^{-}$ & $1.275(85)$ & $3.07(20)$ & $3.63(32)$ & $5.73(50)$ & $7.52(39)$ & $8.02(42)$ & $7.88(42)$ & $6.45(34)$ \\
\hline 2985(10) & $4^{+}$ & $0.0261(66)$ & $0.048(11)$ & $0.087(11)$ & $0.145(17)$ & $0.231(17)$ & $0.317(23)$ & $0.388(32)$ & $0.424(28)$ \\
\hline 3044(10) & $2^{+}+5^{-}$ & $0.0615(77)$ & $0.111(11)$ & $0.112(13)$ & $0.093(14)$ & $0.123(16)$ & $0.141(13)$ & $0.140(18)$ & $0.204(17)$ \\
\hline $3232(10)$ & $\left(3^{-}\right)$ & & - & $0.0080(49)$ & $0.0271(81)$ & $0.0430(56)$ & $0.0541(63)$ & $0.0423(58)$ & $0.0297(48)$ \\
\hline $3364(10)$ & $3^{-}$ & 94(16) & $0.464(33)$ & $0.562(51)$ & $0.811(75)$ & 1.079 & $1.102(60)$ & $1.085(60)$ & $0.910(50)$ \\
\hline $3432(20)$ & $2^{+}$ & $0.0758(84)$ & $0.113(10)$ & $0.126(14)$ & $0.125(17)$ & $0.124(10)$ & $0.0669(82)$ & $0.0734(88)$ & $0.0398(61)$ \\
\hline$\sim 3500$ & $4^{+}+5^{-}$ & $0.0383(66)$ & $0.0958(93)$ & $0.114(12)$ & $0.178(20)$ & $0.240(15)$ & $0.373(23)$ & $0.404(28)$ & $0.479(28)$ \\
\hline $2646(10)$ & $\left(3^{-}\right)$ & $0.0256(71)$ & $0.0444(70)$ & $0.0440(71)$ & $0.096(14)$ & $0.1187(96)$ & $0.142(11)$ & $0.142(12)$ & $0.0965(87)$ \\
\hline $2720(10)$ & $2^{+}$ & $0.0343(77)$ & $0.0787(92)$ & $0.095(11)$ & $0.095(14)$ & $0.0834(83)$ & $0.0660(79)$ & $0.0556(73)$ & $0.0322(56)$ \\
\hline $3989(10)$ & $4^{+}+5^{-}$ & $0.0312(69)$ & $0.0322(95)$ & $0.0234(66)$ & $0.0414(85)$ & $0.0686(82)$ & $0.114(12)$ & $0.108(11)$ & $0.149(11)$ \\
\hline 4052(10) & $2^{+}$ & $0.095(11)$ & $0.150(17)$ & $0.204(22)$ & $0.205(22)$ & $0.209(16)$ & $0.149(14)$ & $0.131(12)$ & $0.0894(80)$ \\
\hline $4166(10)$ & $4^{+}$ & $0.0153(51)$ & $0.0341(72)$ & $0.0519(84)$ & $0.097(13)$ & 0.102 & $0.167(20)$ & $0.158(15)$ & $0.176(16)$ \\
\hline & 5 & & - & - & & $0.051(15)$ & $0.077(18)$ & $0.113(13)$ & $0.142(15)$ \\
\hline $4301(10)$ & 3 & $636(78)$ & $0.112(12)$ & $0.124(14)$ & $0.139(16)$ & $0.156(12)$ & $0.150(13)$ & $0.141(12)$ & $0.1185(92)$ \\
\hline $4473(10)$ & $2^{+}$ & $0.0541(72)$ & $0.103(11)$ & $0.111(13)$ & $0.108(13)$ & - & $0.070(16)$ & $0.065(14)$ & $0.0340(71)$ \\
\hline $4582(10)$ & $\left(3^{-}\right)$ & $0.0222(55)$ & $0.0642(97)$ & $0.0676(97)$ & $0.096(13)$ & $0.124(11)$ & $0.130(12)$ & $0.149(12)$ & $0.1266(96)$ \\
\hline $4660(10)$ & $\left(3^{-}\right)$ & $0.0172(54)$ & $0.0399(86)$ & $0.0591(92)$ & $0.079(12)$ & $0.127(11)$ & $0.153(14)$ & $0.142(12)$ & $0.135(10)$ \\
\hline
\end{tabular}

Table B.9: Cross sections of the ${ }^{70} \mathrm{Zn}\left(\mathrm{p}, \mathrm{p}^{\prime}\right)$ experiment $\left(7.28^{\circ}-16.07^{\circ}\right)$.

\begin{tabular}{|c|c|c|c|c|c|c|c|c|c|}
\hline$E_{\mathrm{x}}(\mathrm{keV})$ & $\mathrm{J}^{\pi}$ & $16.89^{\circ}$ & $18.61^{\circ}$ & $19.48^{\circ}$ & $21.20^{\circ}$ & $21.97^{\circ}$ & $23.69^{\circ}$ & $24.51^{\circ}$ & $26.23^{\circ}$ \\
\hline 0 & $0^{+}$ & $63.38(330)$ & $57.6(30)$ & $51.15(292)$ & $26.69(153)$ & $20.32(228)$ & $8.75(98)$ & $6.88(39)$ & $3.71(21)$ \\
\hline $875(20)$ & $2^{+}$ & $2.18(11)$ & $1.514(80)$ & $1.78(10)$ & $2.17(12)$ & $2.22(25)$ & $1.957(220)$ & $1.907(108)$ & $1.255(71)$ \\
\hline $1761(10)$ & $2^{+}$ & $0.211(16)$ & $0.143(11)$ & $0.124(10)$ & $0.0759(65)$ & $0.0804(98)$ & $0.091(11)$ & $0.0905(73)$ & $0.0836(58)$ \\
\hline 1787 & $4^{+}$ & $0.311(21)$ & $0.201(13)$ & $0.198(14)$ & $0.1347(97)$ & $0.104(12)$ & $0.0732(90)$ & $0.0901(73)$ & $0.0707(52)$ \\
\hline $1958(10)$ & $2^{+}$ & $0.0983(77)$ & $0.0370(28)$ & $0.0326(37)$ & $0.0345(39)$ & $0.0359(46)$ & $0.0434(53)$ & $0.0499(41)$ & $0.0455(32)$ \\
\hline $2667(10)$ & $2^{+}$ & $0.0161(99)$ & - & $0.0044(32)$ & & $0.0043(33)$ & $0.0030(13)$ & $0.0044(17)$ & $0.0040(14)$ \\
\hline 2695(10) & $4^{+}$ & $0.128(14)$ & $0.120(15)$ & $0.111(11)$ & $0.0816(61)$ & $0.0712(92)$ & $0.0493(61)$ & $0.0414(33)$ & $0.0225(21)$ \\
\hline $2865(10)$ & $3^{-}$ & $4.83(26)$ & $3.16(17)$ & $2.35(14)$ & $1.253(73)$ & $0.939(106)$ & $0.670(76)$ & $0.668(38)$ & $0.596(34)$ \\
\hline $2985(10)$ & $4^{+}$ & $0.440(30)$ & $0.429(27)$ & $0.358(26)$ & $0.243(16)$ & $0.206(24)$ & $0.129(15)$ & $0.1189(75)$ & $0.0792(51)$ \\
\hline $3044(10)$ & $2^{+}+5^{-}$ & $0.243(20)$ & $0.296(20)$ & $0.321(26)$ & $0.333(22)$ & $0.321(37)$ & $0.276(32)$ & $0.271(16)$ & $0.203(12)$ \\
\hline $3232(10)$ & $\left(3^{-}\right)$ & $0.0169(33)$ & $0.0048(33)$ & $0.0077(26)$ & $0.0087(20)$ & $0.0079(24)$ & $0.0150(24)$ & $0.0167(18)$ & $0.0162(16)$ \\
\hline $3364(10)$ & $3^{-}$ & $0.671(37)$ & $0.475(27)$ & $0.392(24)$ & $0.271(17)$ & $0.220(25)$ & $0.181(21)$ & $0.180(11)$ & $0.1468(90)$ \\
\hline $3432(20)$ & $2^{+}$ & $0.0223(49)$ & $0.0263(46)$ & $0.0219(41)$ & $0.0106(26)$ & $0.0137(34)$ & $0.0158(36)$ & $0.0103(20)$ & $0.0078(17)$ \\
\hline$\sim 3500$ & $4^{+}+5^{-}$ & $0.513(29)$ & $0.55(12)$ & $0.546(39)$ & $0.494(31)$ & $0.432(48)$ & $0.352(60)$ & $0.330(33)$ & $0.256(40)$ \\
\hline $2646(10)$ & $\left(3^{-}\right)$ & $0.0544(56)$ & $0.0282(38)$ & $0.0251(45)$ & $0.0322(35)$ & $0.0295(46)$ & $0.0427(56)$ & $0.0469(40)$ & $0.0399(31)$ \\
\hline $2720(10)$ & $2^{+}$ & $0.0084(38)$ & - & $0.0096(37)$ & $0.0109(25)$ & $0.0101(28)$ & - & $0.0074(21)$ & $0.0053(17)$ \\
\hline $3989(10)$ & $4^{+}+5^{-}$ & $0.149(11)$ & $0.164(11)$ & $0.167(12)$ & $0.1436(95)$ & $0.114(14)$ & $0.091(11)$ & $0.0815(57)$ & $0.0525(37)$ \\
\hline $4052(10)$ & $2^{+}$ & $0.0517(62)$ & $0.0327(49)$ & $0.0364(48)$ & $0.0288(33)$ & $0.0215(36)$ & $0.0249(37)$ & $0.0235(26)$ & $0.0196(21)$ \\
\hline $4166(10)$ & $4^{+}$ & $0.151(13)$ & $0.116(11)$ & $0.1039(92)$ & $0.0787(71)$ & $0.0542(80)$ & $0.0326(52)$ & $0.0346(39)$ & $0.0219(27)$ \\
\hline $4210(10)$ & $5^{-}$ & $0.188(15)$ & $0.232(16)$ & $0.224(16)$ & $0.184(12)$ & $0.179(21)$ & $0.143(17)$ & $0.1311(88)$ & $0.0912(61)$ \\
\hline $4301(10)$ & $3^{-}$ & $0.0827(80)$ & $0.045(15)$ & $0.0504(61)$ & $0.0316(71)$ & $0.0272(63)$ & $0.0316(56)$ & $0.0261(51)$ & $0.0177(70)$ \\
\hline $4473(10)$ & $2^{+}$ & $0.0177(50)$ & - & - & $0.0120(26)$ & $0.0149(30)$ & $0.0153(28)$ & $0.0174(23)$ & $0.0112(16)$ \\
\hline $4582(10)$ & $\left(3^{-}\right)$ & $0.113(10)$ & $0.0960(77)$ & $0.0602(81)$ & $0.0304(60)$ & $0.0378(64)$ & $0.0300(46)$ & $0.0303(40)$ & $0.0302(26)$ \\
\hline $4660(10)$ & $\left(3^{-}\right)$ & $0.1108(99)$ & $0.0824(74)$ & $0.0644(64)$ & $0.0408(40)$ & $0.0322(47)$ & $0.0175(32)$ & $0.0240(28)$ & $0.0182(22)$ \\
\hline
\end{tabular}

Table B.10: Cross sections of the ${ }^{70} \mathrm{Zn}\left(\mathrm{p}, \mathrm{p}^{\prime}\right)$ experiment $\left(16.89^{\circ}-26.23^{\circ}\right)$. 


\section{Bibliography}

[AFR] AFRODITE webpage, http://www.tlabs.ac.za/nuclear-physics-afrodite/

[Ago03] S. Agostinelli, J. Allison, K. Amako, J. Apostolakis, H. Araujo, P. Arce, M. Asai, D. Axen, S. Banerjee, G. Barrand, F. Behner, L. Bellagamba, J. Boudreau, L. Broglia, A. Brunengo, H. Burkhardt, S. Chauvie, J. Chuma, R. Chytracek, G. Cooperman, G. Cosmo, P. Degtyarenko, A. Dell'Acqua, G. Depaola, D. Dietrich, R. Enami, A. Feliciello, C. Ferguson, H. Fesefeldt, G. Folger, F. Foppiano, A. Forti, S. Garelli, S. Giani, R. Giannitrapani, D. Gibin, J. J. Gomez Cadenas, I Gonzalez, G. Gracia Abril, G. Greeniaus, W. Greiner, V. Grichine, A. Grossheim, S. Guatelli, P. Gumplinger, R. Hamatsu, K. Hashimoto, H. Hasui, A. Heikkinen, A. Howard, V. Ivanchenko, A. Johnson, F. W. Jones, J. Kallenbach, N. Kanaya, M. Kawabata, Y. Kawabata, M. Kawaguti, S. Kelner, P. Kent, A. Kimura, T. Kodama, R. Kokoulinm, M. Kossov, H. Kurashige, E. Lamanna, T. Lampen, V. Lara, V. Lefebure, F. Lei, M. Liendl, W. Lockman, F. Longo, S. Magni, M. Maire, E. Medernach, K. Minamimoto, P. Mora de Freitas, Y. Morita, K. Murakami, M. Nagamatu, R. Nartallo, P. Nieminen, T. Nishimura, K. Ohtsubo, M. Okamura, S. O'Neale, Y. Oohata, K. Paech, J. Perl, A. Pfeiffer, M. G. Pia, F. Ranjard, A. Rybin, S. Sadilov, E. Di Salvo, G. Santin, T. Sasaki, N. Savvas, Y. Sawada, S. Scherer, S. Sei, V. Sirotenko, D. Smith, N. Starkov, H. Stoecker, J. Sulkimo, M. Takahata, S. Tanaka, E. Tcherniaev, E. Safai Tehrani, M. Tropeano, P. Truscott, H. Uno, L. Urban, P. Urban, M. Verderi, A. Walkden, W. Wander, H. Weberm, J. P. Wellisch, T. Wenaus, D. C. Williams, D. Wright, T. Yamada, H. Yoshida, and D. Zschiesche. Nuclear Physics A 506, 250 (2003).

[Ali12] B. Alikhani, A. Givechev, A. Heinz, P. R. John, J. Leske, M. Lettmann, O. Möller, N. Pietralla, and C. Röder, Nuclear Instruments and Methods in Physics Research Section A 675, 144 (2012).

[Amo67] K. A. Amos, V. A. Madsen, and I. E. McCarthy, Nuclear Physics A 94, 103 (1967).

[Ari75] A. Arima and F. Iachello, Phys. Rev. Lett. 35, 1069 (1975).

[Ari78] A. Arima and F. Iachello, Phys. Rev. Lett. 40, 385 (1978).

[Bar57] J. Bardeen, L. N. Cooper, and J. R. Schrieffer, Phys. Rev. Lett. 108, 1175 (1957).

[Ber99] C. A. Bertulani and V. Yu. Ponomarev, Physics Reports 321, 139 (1999).

[Bog03] S. K. Bogner, T. T. S. Kuo, and A. Schwenk, Physics Reports 386, 1 (2003).

[Bre74] A. Breskin, G. Charpak, B. Gabioud, F. Sauli, N. Trautner, W. Duinker, and G. Schultz, Nuclear Instruments and Methods 119, 9 (1974).

[Bro05] B. A. Brown, N. J. Stone, J. R. Stone, I. S. Towner, and M. Hjorth-Jensen, Phys. Rev. C 71, 044317 (2005). 
[Bur07] O. Burda, N. Botha, J. Carter, R. W. Fearick, S. V. Förtsch, C. Fransen, H. Fujita, J. D. Holt, M. Kuhar, A. Lenhardt, P. von Neumann-Cosel, R. Neveling, N. Pietralla, V. Yu. Ponomarev, A. Richter, O. Scholten, E. Sideras-Haddad, F. D. Smit, and J. Wambach, Phys. Rev. Lett. 99, 092503 (2007).

[Bur57] E. M. Burbidge, G. R. Burbidge, W. A. Fowler, and F. Hoyle, Rev. Mod. Phys. 29, 547 (1957).

[Cas13] R. J. Casperson, V. Werner, and S. Heinze, Physics Letters B 721, 51 (2013).

[Els89] Ch. Elster and P. C. Tandy, Phys. Rev. C 40, 881 (1989).

[Elh08] E. Elhami, J. N. Orce, M. Scheck, S. Mukhopadhyay, S. N. Choudry, M. T. McEllistrem, S. W. Yates, C. Angell, M. Boswell, B. Fallin, C. R. Howell, A. Hutcheson, H. J. Karwowski, J. H. Kelley, Y. Parpottas, A. P. Tonchev, and W. Tornow, Phys. Rev. C 78, 064303 (2008).

[Elh13a] E. Elhami, J. N. Orce, M. Scheck, S. Mukhopadhyay, S. N. Choudry, M. T. McEllistrem, S. W. Yates, C. Angell, M. Boswell, B. Fallin, C. R. Howell, A. Hutcheson, H. J. Karwowski, J. H. Kelley, Y. Parpottas, A. P. Tonchev, and W. Tornow, Phys. Rev. C 88, 029903 (2013).

[Elh13b] E. Elhami, J. N. Orce, S. Mukhopadhyay, S. N. Choudry, M. Scheck, M. T. McEllistrem, and S. W. Yates, Phys. Rev. C 88, 029902 (2013).

[Epe09] E. Epelbaum, H.-W. Hammer, and Ulf-G. Meißner, Rev. Mod. Phys. 81, 1173 (2009).

[FAI]

FAIR webpage, http://www.gsi.de/en/research/fair.htm

[Fel98]

H. Feldmeier, T. Neff, R. Roth, and J. Schnack, Nuclear Physics A 632, 61 (1998).

[Fes58] H. Feshbach, Annals of Physics 5, 357 (1958).

[Fra03] C. Fransen, N. Pietralla, Z. Ammar, D. Bandyopadhyay, N. Boukharouba, P. von Brentano, A. Dewald, J. Gableske, A. Gade, J. Jolie, U. Kneissl, S. R. Lesher, A. F. Lisetskiy, M. T. McEllistrem, M. Merrick, H. H. Pitz, N. Warr, V. Werner, and S. W. Yates, Phys. Rev. C 67, 024307 (2003).

[Fra05] C. Fransen, V. Werner, D. Bandyopadhyay, N. Boukharouba, S. R. Lesher, M. T. McEllistrem, and J. Jolie, N. Pietralla, P. von Brentano, and S. W. Yates, Phys. Rev. C 71, 054304 (2005).

[FRI] FRIB webpage, http://www.frib.msu.edu

[Fri75] J. L Friar, Annals of Physics 95, 170 (1975).

[Fre87] E. Fretwurst, G. Lindström, K. F. von Reden, V. Riech, S. I. Vasiljev, P. P. Zarubin, O. M. Knyazkov, and I. N. Kuchtina, Nuclear Physics A 469, 247 (1987).

[Gaf13] L. P. Gaffney, P. A. Butler, M. Scheck, and A. B. Hayes, Nature 497, 199 (2013).

[Gaz83] M. M. Gazzaly, N. M. Hintz, M. A. Franey, J. Dubach, and W. C. Haxton, Phys. Rev. C 28, 294 (1983). 
[Geo82] K. Goeke and J. Speth, Annual Review of Nuclear and Particle Science 32, 65 (1982).

[Ger71] H. V. Geramb and K. A. Amos, Nuclear Physics A 163, 337 (1971).

[Goe30] M. Goeppert-Mayer, Dissertation (1930).

[Gol53] A. de-Shalit and M. Goldhaber Phys. Rev. 92, 1211 (1953).

[GSI] GSI webpage, http://www.gsi.de

[Gre62] D. P. Grechukhin, Nuclear Physics 35, 98 (1962).

[Gre63] D. P. Grechukhin, Nuclear Physics 47, 273 (1963).

[Gre65] D. P. Grechukhin, Nuclear Physics 62, 273 (1965).

[Hag12] G. Hagen and N. Michel, Phys. Rev. C 86, 021602 (2012).

[Ham84] W. D. Hamilton, A. Irbäck, and J. P. Elliott, Phys. Rev. Lett. 53, 2469 (1984).

[Hey86] K. Heyde, and J. Sau, Phys. Rev. C 33, 1050 (1986).

[Hof03] F. Hofmann, Dissertation (2003).

[Hud72] F. R. Hudson, and R. N. Glover, Nuclear Physics A 189, 264 (1972).

[Hup13] G. Hupin, J. Langhammer, P. Navrátil, S. Quaglioni, A. Calci, R. Roth, Phys. Rev. C 88, 054622 (2013).

[Iac87] F. Iachello, and A. Arima, The Interacting Boson Model, Cambridge University Press, Cambridge (1987).

[Iac00] F. Iachello, Phys. Rev. Lett. 85, 3580 (2000).

[Ila06] K. Ilakovac, M. Uroic, M. Majer, S. Pasic, and B. Vukovic, Radiation Physics and Chemistry 75, 1451 (2006).

[Isa86] P. Van Isacker, K. Heyde, J. Jolie, and A. Sevrin, Annals of Physics 171, 253 (1986).

[Isa02] V. I. Isakov, K. I. Erokhina, H. Mach, M. Sanchez-Vega, and B. Fogelberg Eur. Phys. J. A 14, 29 (2002).

[ISO] ISOLDE webpage, http://isolde.web.cern.ch/rex-isolde

[iTH] iThemba webpage, http://www.tlabs.ac.za

[Iud04] N. Lo Iudice, and Ch. Stoyanov, Phys. Rev. C 69, 044312 (2004).

[Jab87] J. Jabbour, L. H. Rosier, B. Ramstein, R. Tamisier, and P. Avignon Nuclear Physics A 646, 287 (1987).

[Jac70] D. F. Jackson, Nuclear Reactions, Methuen and Co LTD (1970). 
[Jin96] D. S. Jin, J. R. Ensher, M. R. Matthews, C. E. Wieman, and E. A. Cornell, Phys. Rev. Lett. 77, 420 (1996).

[Jol71] R. K. Jolly and E. Kashy, Phys. Rev. C 4, 1398 (1971).

[Kar95] S. Karataglidis, P. J. Dortmans, K. Amos, and R. de Swiniarski Phys. Rev. C 52, 861 (1995).

[Kim96] K. H. Kim, T. Otsuka, P. von Brenato, A. Gelberg, P. van Isacker,R. F. Casten, in: G. Molnar (Ed.), Capture Gamma-Ray Spectroscopy and Related Topics, vol I, Springer, Budapest, 1996, p. 195.

[Kno10] G. F. Knoll, Radiation detection and measurement, Wiley (2010)

[Kow04] K. Kowalski, D. J. Dean, M. Hjorth-Jensen, T. Papenbrock, and P. Piecuch, Phys. Rev. Lett. 92, 132501 (2004).

[Kni91] M. Knirsch, Dissertation (1991).

[Kra87] J. Kramp, D. Habs, R. Kroth, M. Music, J. Schirmer, D. Schwalm, and C. Broude, Nuclear Physics A 474, 412 (1987).

[Kun] P. D. Kunz, computer code CHUCK3, unpublished.

[Leo89] R. De Leo, L. Lagamba, N. Blasi, S. Micheletti, M. Pignanelli, M. Fujiwara, K. Hosono, I. Katayama, N. Matsuoka, S. Morinobu, T. Noro, S. Matsuki, H. Okamura, J. M. Schippers, S. Y. Van Der Werf, and M. N. Harakeh, Physics Letters B 226, 5 (1989).

[Leo98] R. De Leo, H. Akimune, N. Blasi, I. Daito, Y. Fujita, M. Fujiwara, S. I. Hayakawa, S. Hatori, H. Ikegami, T. Inomata, I. Katayama, K. Katori, L. Lagamba, S. Micheletti, S. Morinobu, R. Perrino, M. Pignanelli, H. Sakamoto, J. Takamatsu, and M. Tosaki, Phys. Rev. C 57, 1604 (1998).

[Leo01] E. V. D. van Loef, P. Dorenbos, C. W. E. van Eijk, K. Krämer, and H. U. Güdel, Appl. Phys. Lett. 79, 1573 (2001).

[Lev63] F. S. Levin, Nuclear Physics 46, 275 (1963).

[Les07] S. R. Lesher, C. J. McKay, M. Mynk, D. Bandyopadhyay, N. Boukharouba, C. Fransen, J. N. Orce, M. T. McEllistrem, and S. W. Yates, Phys. Rev. C 75, 034318 (2007).

[Lov81] W. G. Love and M. A. Franey, Phys. Rev. C 24, 1073 (1981).

[Lov85] W. G. Love and M. A. Franey, Phys. Rev. C 31, 488 (1985).

[Les07] S. R. Lesher, C. J. McKay, M. Mynk, D. Bandyopadhyay, N. Boukharouba, C. Fransen, J. N. Orce, M. T. McEllistrem, and S. W. Yates, Phys. Rev. C 75, 034318 (2007).

[Lue95] C. Lüttge, C. Hofmann, J. Horn, F. Neumeyer, A. Richter, G. Schrieder, E. Spamer, A. Stiller, D. I. Sober, S. K. Matthews, and L. W. Fagg, Nuclear Instruments and Methods in Physics Research Section A 366, 325 (1995). 
[Mac01] R. Machleidt, Phys. Rev. C 63, 024001 (2001).

[Mac11] R. Machleidt and D. R. Entem, Physics Reports 503, 1 (2011).

[Man01] P. F. Mantica, A. E. Stuchbery, D. E. Groh, J. I. Prisciandaro, and M. P. Robinson, Phys. Rev. C 63, 034312 (2001).

[Mar14] D. Martin, private communication (2014).

[Met83] V. Metag, R. D. Fischer, W. Kühn, R. Mühlhans, R. Novotny, D. Habs, U. v. Helmolt, H. W. Heyng, R. Kroth, D. Pelte, D. Schwalm, W. Hennerici, H. J. Hennrich, G. Himmele, E. Jaeschke, R. Repnow, W. Wahl, E. Adelberger, A. Lazzarini, R. S. Simon, R. Albrecht, and B. Kolb, Nuclear Physics A 409, 311 (1983).

[MID07] Maximum Integrated Data Acquisition System, Paul Scherrer Institute, TRIUMF unpublished (2007)

[Mil11] D. J. Millener, R. J. Sutter, and D. E. lburger, 2- $\gamma$ decay of the 662-keV isomer in ${ }^{137} \mathrm{Ba}, 2011$ Fall Meeting of the APS Division of Nuclear Physic (2011).

[Mil13a] D. J. Millener, Theory for $2-\gamma$ transitions: I, unpublished (2013).

[Mil13b] D. J. Millener, Theory for 2- $\gamma$ transitions: II, unpublished (2013).

[Mil14] D. J. Millener, private communication (2014).

[Mue09] D. Mücher, Dynamische Symmetrien von Atomkernen an Unterschaöenabschlüssen, Dissertation (2009).

[Mok04] P. H. Mokler and R. W. Dunford, Phys. Scr. 69, C1 (2004).

[Mus88] M. Music, Dissertation (1988).

[Nav00] P. Navrátil, J. P. Vary, and B. R. Barrett, Phys. Rev. C 62, 054311 (2000).

[Nef03] T. Neff and H. Feldmeier, Nuclear Physics A 713, 311 (2003).

[Nev01] R. Neveling, Nuclear medium effects on analyzing power investigated with a proton knockout reaction, PhD thesis, Stellenbosch (2001).

[Nev11] R. Neveling, F. D. Smit, H. Fujita, and R. T. Newman, K600 user manual (2012).

[Nev11] R. Neveling, H. Fujita, F. D. Smit, T. Adachi, G. P.A. Berg, E. Z. Buthelezi, J. Carter, J. L. Conradie, M. Couder, R. W. Fearick, S. V. Förtsch, D. T. Fourie, Y. Fujita, J. Görres, K. Hatanaka, M. Jingo, A. M. Krumbholz, C. O. Kureba, J. P. Mira, S. H. T. Murray, P. von Neumann-Cosel, S. O'Brien, P. Papka, I. Poltoratska, A. Richter, E. Sideras-Haddad, J. A. Swartz, A. Tamii, I. T. Usman, and J. J. van Zyl, Nuclear Instruments and Methods in Physics Research Section A 654, 29 (2011).

[New96] R. T. Newton, Single Proton Transfer on ${ }^{55} \mathrm{Mn}$, PhD thesis, University of Cape Town (1996). 
[Nak91] Hitoshi Nakada, Takaharu Otsuka, and Takashi Sebe, Phys. Rev. Lett. 67, 1086 (1991).

[NSC] NSCL webpage, http://www.nscl.msu.edu

[Nup04] NuPECC Long Range Plan (2004)

[Nup10] NuPECC Long Range Plan (2010)

[Ots78] T. Otsuka, A. Arima, and F. Iachello, Nuclear Physics A 309, 1 (1978).

[Ots85] Takaharu Otsuka, and Joseph N. Ginocchio, Phys. Rev. Lett. 54, 777 (1985).

[Ots94] Takaharu Otsuka, and Ka-Hae Kim, Phys. Rev. C 50, R1768 (1994).

[Pap85] C. N. Papanicolas, S. E. Williamson, H. Rothhaas, G. O. Bolme, L. J. Koester, B. L. Miller, R. A. Miskimen, P. E. Mueller, and L. S. Cardman, Phys. Rev. Lett. 54, 26 (1985).

[Pel82] D. Pelte and D. Schwalm, In-beam gamma-ray spectroscopy with heavy ions, North-Holland Publishing Company (1982).

[Pie05] S. C. Pieper, Nuclear Physics A 751, 516 (2005).

[Pie99] N. Pietralla, C. Fransen, D. Belic, P. von Brentano, C. Frießner, U. Kneissl, A. Linnemann, A. Nord, H. H. Pitz, T. Otsuka, I. Schneider, V. Werner, and I. Wiedenhöver, Phys. Rev. Lett. 83, 1303 (1999).

[Pie08] N. Pietralla, P. von Brentano, and A. F. Lisetskiy, Progress in Particle and Nuclear Physics 60, 225 (2008).

[Pig88] M. Pignanelli, S. Micheletti, N. Blasi, R. De Leo, W. T. A. Borghols, J. M. Schippers, S. Y. Van Der Werf, and M. N. Harakeh, Physics Letters B 202, 470 (1988).

[Pig92] M. Pignanelli, N. Blasi, S. Micheletti, R. De Leo, L. LaGamba, R. Perrino, J. A. Bordewijk, M. A. Hofstee, J. M. Schippers, S. Y. van der Werf, J. Wesseling, and M. N. Harakeh, Nuclear Physics A 540, 27 (1992).

[Pon14] V. Yu. Ponomarev, private communication (2014).

[Pol11] I. Poltoratska, Complete dipole repsonse in ${ }^{208} \mathrm{~Pb}$ from high-resolution polarized proton scattering at $0^{\circ}, \mathrm{PhD}$ thesis, Darmstadt (2011).

[Ray07a] J. Raynal, computer code DWBA07, NEA 1209/02 (2007).

[Ray07b] J. Raynal, notes on DWBA98, unpublished.

[Rei10] P.-G. Reinhard and W. Nazarewicz, Phys. Rev. C 81, 051303 (2010).

[Rie] F. Riess, Gaspan program, Sektion Physik, Ludwig-Maximilians-Universität München, unpublished.

[Rik] RIKEN webpage, http://www.riken.jp/en/

[Rik84] L. Rikus, K. Nakano, and H. V. Von Geramb, Nuclear Physics A 414, 413 (1984). 
[ROT] root webpage, http://root.cern.ch/drupal/

[Rot07] R. Roth and P. Navrátil, Phys. Rev. Lett. 99, 092501 (2007).

[Rot11] R. Roth, International Workshop on Nuclear Physics 2011, Stellenbosch Institute for Advanced Studies (2011).

[Sag87] H. Sagawa, O. Scholten, B. A. Brown, and B. H. Wildenthal, Nuclear Physics A 462, 1 (1987).

[Sai] BrilLanCe Scintillators Performance Summary, Available from: http://www.detectors.saint-gobain.com

[Sav10] D. Savran, K. Lindenberg, J. Glorius, B. Löher, S. Müller, N. Pietralla, L. Schnorrenberger, V. Simon, K. Sonnabend, C. Wälzlein, M. Elvers, J. Endres, J. Hasper, and A. Zilges, Nuclear Instruments and Methods in Physics Research Section A 613, 232 (2010).

[Sch10] M. Scheck, P. A. Butler, C. Fransen, V. Werner, and S. W. Yates, Phys. Rev. C 81, 064305 (2010).

[Sch13] A. Scheikh-Obeid, O. Burda, M. Chernykh, A. Krugmann, P. von Neumann-Cosel, N. Pietralla, I. Poltoratska, V. Yu. Ponomarev, and C. Walz, Phys. Rev. C 87, 014337 (2013).

[Sch84] J. Schirmer, D. Habs, R. Kroth, N. Kwong, D. Schwalm, M. Zirnbauer, and C. Broude, Phys. Rev. Lett. 53, 1897 (1984).

[She05] A. Shevchenko, Fine Structure of the Isoscalar Giant Quadrupole Resonance from High-Resolution Inelastic Proton Scattering Experiments, PhD thesis, Darmstadt (2005).

[Sie09] K. Sieja, F. Nowacki, K. Langanke, and G. Martinez-Pinedo, Phys. Rev. C 79, 064310 (2009).

[Sin86] P. Singh, D. Rychel, G. Gyufko, B. van Krüchten, M. Lahanas, and C. A. Wiedner, Nuclear Physics A 458, 1 (1986).

[Sol92] V.G. Soloviev, Theory of Atomic Nuclei: Quasiparticles and Phonons, Institute of Physics Publishing, Bristol (1992)

[SPI] SPIRAL2 webpage, http://pro.ganil-spiral2.eu/spiral2

[Tam09] A. Tamii, Y. Fujita, H. Matsubara, T. Adachi, J. Carter, M. Dozono, H. Fujita, K. Fujita, H. Hashimoto, K. Hatanaka, T. Itahashi, M. Itoh, T. Kawabata, K. Nakanishi, S. Ninomiya, A. B. Perez-Cerdan, L. Popescu, B. Rubio , T. Saito, H. Sakaguchi, Y. Sakemi, Y. Sasamoto, Y. Shimbara, Y. Shimizuand, F. D. Smit, Y. Tameshige, M. Yosoi, and J. Zenhiro, Nuclear Instruments and Methods in Physics Research Section A 605, 326 (2009).

[Tam11] A. Tamii, I. Poltoratska, P. von Neumann-Cosel, Y. Fujita, T. Adachi, C. A. Bertulani, J. Carter, M. Dozono, H. Fujita, K. Fujita, K. Hatanaka, D. Ishikawa, 
M. Itoh, T. Kawabata, Y. Kalmykov, A. M. Krumbholz, E. Litvinova, H. Matsubara, K. Nakanishi, R. Neveling, H. Okamura, H. J. Ong, B. Özel-Tashenov, V. Yu. Ponomarev, A. Richter, B. Rubio, H. Sakaguchi, Y. Sakemi, Y. Sasamoto, Y. Shimbara, Y. Shimizu, F. D. Smit, T. Suzuki, Y. Tameshige, J. Wambach, R. Yamada, M. Yosoi, and J. Zenihiro, Phys. Rev. Lett. 107, 062502 (2011).

[Tan85] I. Tanihata, H. Hamagaki, O. Hashimoto, Y. Shida, N. Yoshikawa, K. Sugimoto, O. Yamakawa, T. Kobayashi, and N. Takahashi, Phys. Rev. Lett. 55, 2676 (1985).

[Tew13] I. Tews, T. Krüger, K. Hebeler, and A. Schwenk, Phys. Rev. Lett. 110, 032504 (2013).

[Tul04] J. K. Tuli, Nuclear Data Sheets 103, 389 (2004).

[Joh10] H. T. Johansson, The ucesb unpacker generator (2010).

[Vri87] H. De Vries, C. W. De Jager, and C. De Vries, Atomic Data and Nuclear Data Tables 36, (1987).

[Wal10] C. Walz, Master thesis, (2010).

[Wam10] J. Wambach, private communication (2010).

[Wal11] C. Walz, H. Fujita, A. Krugmann, P. von Neumann-Cosel, N. Pietralla, V. Yu. Ponomarev, A. Scheikh-Obeid, and J. Wambachm, Phys. Rev. Lett. 106, 062501 (2011).

[Wer08] V. Werner, N. Benczer-Koller, G. Kumbartzki, J. D. Holt, P. Boutachkov, E. Stefanova, M. Perry, N. Pietralla, H. Ai, K. Aleksandrova, G. Anderson, R. B. Cakirli, R. J. Casperson, R. F. Casten, M. Chamberlain, C. Copos, B. Darakchieva, S. Eckel, M. Evtimova, C. R. Fitzpatrick, A. B. Garnsworthy, G. Gürdal, A. Heinz, D. Kovacheva, C. Lambie-Hanson, X. Liang, P. Manchev, E. A. McCutchan, D. A. Meyer, J. Qian, A. Schmidt, N. J. Thompson, E. Williams, and R. Winkler, Phys. Rev. C 78, 031301 (2008).

[Wir95] R. B. Wiringa, V. G. J. Stoks, and R. Schiavilla, Phys. Rev. C 51, 38 (1995).

[Zyl12] JJ Van Zyl, Two-nucleon transfer in the ${ }^{58} \mathrm{Ni}\left(\mathrm{p},{ }^{3} \mathrm{He}\right){ }^{56} \mathrm{Co}$ reaction at incident energies of 80, 100 and $120 \mathrm{MeV}$, PhD thesis, Stellenbosch (2012). 


\section{List of Figures}

1.1. Theoretical approaches to solve the nuclear many-body problem. . . . . . . 1

1.2. The two-photon decay in atomic and nuclear physics. . . . . . . . . . . 3

1.3. Known one-phonon quadrupole mixed-symmetry states. . . . . . . . . . . 6

2.1. Feynman diagrams of the amplitudes contributing to the one- and two-photon

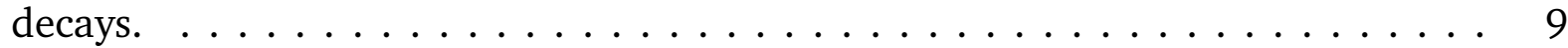

2.2. Simple model of the two-photon decay of the $1 h_{11 / 2}^{-}$isomer in ${ }^{137} \mathrm{Ba} . \ldots \ldots \ldots 13$

2.3. Energy and angular dependence of the two-photon decay probability. . . . . . . . 15

3.1. 'Direct' and 'sequential' Compton scattering. . . . . . . . . . . . . . . . . . . 18

3.2. Energy spectrum of 'sequential' Compton scattering for a relative detector angle of $72^{\circ} \ldots \ldots \ldots \ldots \ldots$. . . . . . . . . . . . . . . . . . 19

3.3. Experimental setup of the two-photon experiment. . . . . . . . . . . . . 19

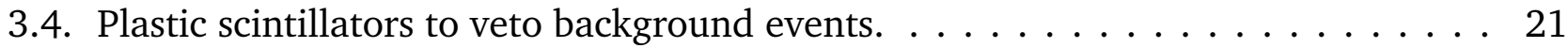

3.5. Energy and time spectra of the background. . . . . . . . . . . . . 22

3.6. Electronic setup of the two-photon experiment. . . . . . . . . . . . . . 24

4.1. Long-term stability of the energy calibration and efficiency curve of one $\mathrm{LaBr}_{3}$ -

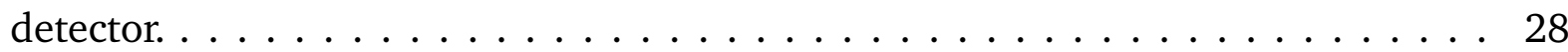

4.2. Time spectrum of all detector pairs with an angle of $144^{\circ}$ and time spectrum of a ${ }^{60}$ Co measurement. . . . . . . . . . . . . . . . . . . . . . . .

4.3. Sum energy spectra of all events satisfying the random and true coincidence time

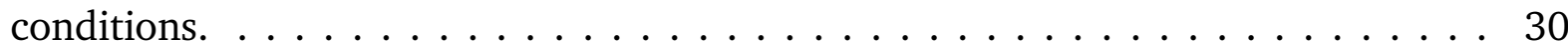

4.4. Background conditions for the two-photon experiment. . . . . . . . . . 31

4.5. Random subtracted sum energy spectrum for an angles of $72^{\circ} \ldots \ldots \ldots 32$

4.6. Impact of the 'sequential' Compton scattering process. . . . . . . . . . . . . . 34

4.7. Random subtracted sum energy spectrum for an angle of $144^{\circ}$ and angular de-

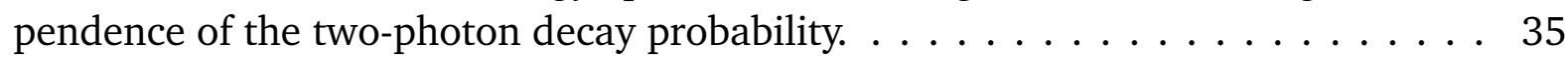

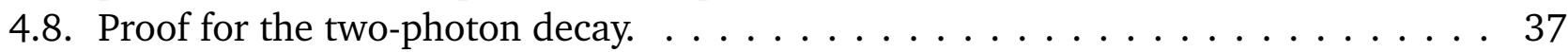

4.9. Energy dependence of the two-photon decay probability. . . . . . . . . . . 38

4.10. Random subtracted sum energy spectra for an angles of $72^{\circ}$ with various energy conditions. . . . . . . . . . . . . . . . . . . . . . . . 39

5.1. Optical potential of ${ }^{92} \mathrm{Zr}$ obtained after folding the effective interaction with the ground state densities. . . . . . . . . . . . . . . . . . 45

5.2. The low-lying states and their decay properties of a spherical nucleus in the framework of the IBM-2. . . . . . . . . . . . . . . . . . . . . . .

5.3. The single particle spectra and the occupation numbers of ${ }^{92} \mathrm{Zr}$ for neutrons and

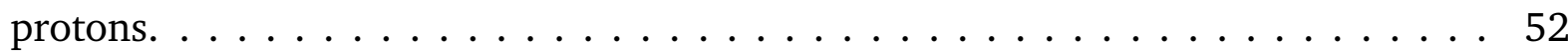

5.4. Two-quasiparticle spectra of ${ }^{92} \mathrm{Zr}$ for neutrons and protons. . . . . . . . . . . 52

5.5. Comparison of the QRPA and QPM spectra of the lowest $2^{+}$states of ${ }^{92} \mathrm{Zr}$. . . . . 54 


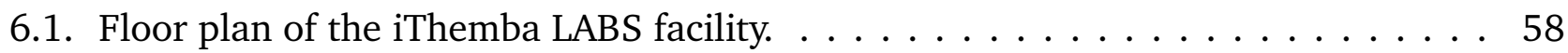

6.2. The K600 spectrometer. . . . . . . . . . . . . . . . . . . . . . . . . . . 59

6.3. Photograph of the K600 spectrometer. . . . . . . . . . . . . . . . . . . . 59

6.4. Cut-out illustration of a vertical drift chamber used in the proton scattering ex-

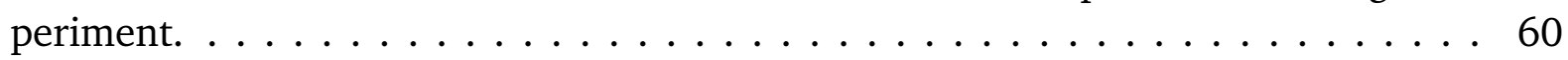

6.5. Electronic setup of the proton scattering experiment. . . . . . . . . . 61

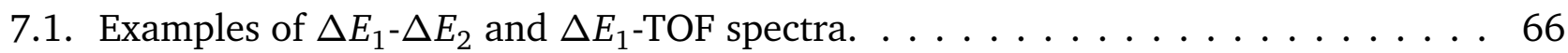

7.2. Schematic of a vertical drift chamber. . . . . . . . . . . . . . . 67

7.3. Drift-time spectrum and associated lookup table of the $X_{2}$-wire plane. . . . . . . 69

7.4. The lookup table shift. . . . . . . . . . . . . . . . . . . . 70

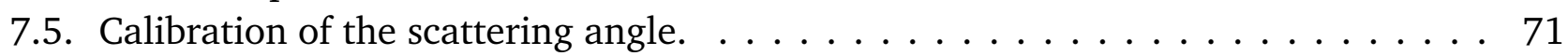

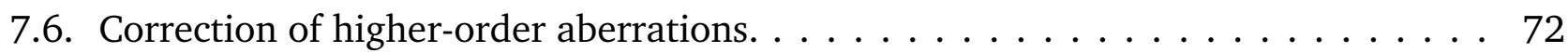

7.7. Excitation spectra of the ${ }^{96} \mathrm{Mo}\left(p, p^{\prime}\right)$ experiment. . . . . . . . . . . . . 73

7.8. Examples of energy spectra of ${ }^{92,94} \mathrm{Zr}$, ${ }^{94,96} \mathrm{Mo}$ and ${ }^{70} \mathrm{Zn}$ in the energy range 0.6 -

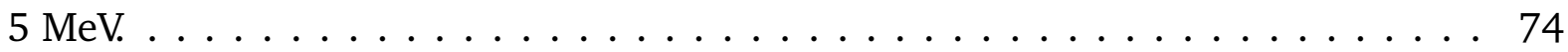

7.9. Normalization problem of the absolute proton scattering cross sections. . . . . . 76

7.10. Angular distributions of the ${ }^{92} \operatorname{Zr}\left(p, p^{\prime}\right)$ experiment. . . . . . . . . . 78

7.11.Angular distributions of the ${ }^{92} \operatorname{Zr}\left(p, p^{\prime}\right)$ experiment. . . . . . . . . . 79

7.12. Angular distributions of the ${ }^{94} \operatorname{Zr}\left(p, p^{\prime}\right)$ experiment. . . . . . . . . . 81

7.13. Angular distributions of the ${ }^{94} \mathrm{Mo}\left(p, p^{\prime}\right)$ experiment. . . . . . . . . . 84

7.14. Angular distributions of the ${ }^{94} \mathrm{Mo}\left(p, p^{\prime}\right)$ experiment. . . . . . . . . . 85

7.15. Angular distributions of the ${ }^{96} \mathrm{Mo}\left(p, p^{\prime}\right)$ experiment. . . . . . . . . 88

7.16. Angular distributions of the ${ }^{96} \mathrm{Mo}\left(p, p^{\prime}\right)$ experiment. . . . . . . . . . . 89

7.17.Angular distributions of the ${ }^{70} \mathrm{Zn}\left(p, p^{\prime}\right)$ experiment. . . . . . . . . . 92

7.18. Angular distributions of the ${ }^{70} \mathrm{Zn}\left(p, p^{\prime}\right)$ experiment. . . . . . . . . . 93

8.1. Comparison of the calculated and measured root-mean-square radii of the charge

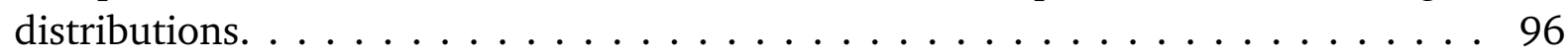

8.2. Comparison of the calculated and measured elastic proton scattering cross sections. 97

8.3. Two-state mixing model. . . . . . . . . . . . . . . . . . . . . 98

8.4. Comparison of the calculated and measured proton scattering cross sections of $2^{+}$states. . . . . . . . . . . . . . . . . . . . . . . . . 103

8.5. Comparison of the calculated and measured electron scattering cross sections of $2^{+}$states. . . . . . . . . . . . . . . . . . . . . . . . 104

8.6. Comparison of the calculated and measured proton scattering cross sections of hexadecapole states. . . . . . . . . . . . . . . . . . . . . . . 107

8.7. Comparison of the calculated and measured electron scattering cross sections of hexadecapole states. . . . . . . . . . . . . . . . . . . . . . . . 108

8.8. Comparison of the calculated and measured proton scattering cross sections of

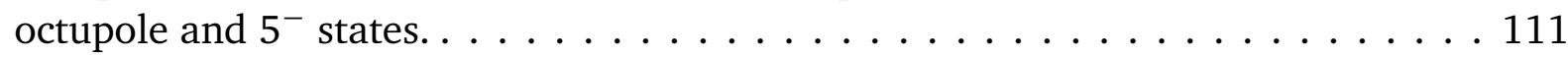

8.9. Comparison of the calculated and measured electron scattering cross sections of octupole states.

8.10. Unperturbed phonon- and two-quasiparticle-energies in ${ }^{94}$ Mo. . . . . . . . . . 113

8.11. Angular distribution of the mixed-symmetry quadrupole two-phonon state. . . . 115

8.12. Running sum of the $R$-value of ${ }^{92} \mathrm{Zr}$. . . . . . . . . . . . . . . . 117 
8.13. Running sums of the $B(E 2)$-values of the lowest quadrupole QRPA-phonons in ${ }^{92} \mathrm{Zr} .118$

8.14. Radial wave functions of four neutron single-particle states. . . . . . . . . . . . 119

8.15. Running sums of the $B(E 4)$-values of the lowest hexadecapole QRPA-phonons in

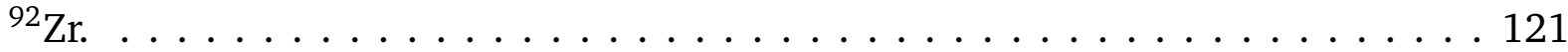

8.16. Running sums of the $B(E 3)$-values of the lowest octupole QRPA-phonons in ${ }^{92} \mathrm{Zr} .{ }^{121}$

8.17. Cross sections of the $2_{1}^{+}$and $2_{\mathrm{ms}}^{+}$states in ${ }^{92,94} \mathrm{Zr}$ and ${ }^{94} \mathrm{Mo} . \ldots \ldots \ldots . \ldots 122$

8.18. Illustration of the dependence of the cross sections of the mixed-symmetry states on the sign of the $\left(2 d_{5 / 2} \otimes 2 d_{5 / 2}\right)_{n}$-two-quasiparticle-state. . . . . . . . . . 122

8.19. Proton and neutron transition densities weighted with $r^{4}$ of ${ }^{94} \mathrm{Mo}$ and ${ }^{92} \mathrm{Zr} . \ldots 124$ 8.20. Proton and neutron transition densities weighted with $r^{4}$ of ${ }^{94} \mathrm{Zr}$. . . . . . . . . 125

8.21. Comparison of the cross sections of all measured $2^{+}$states of ${ }^{92} \mathrm{Zr}$ and ${ }^{94} \mathrm{Mo} . \ldots 126$ 


\section{List of Tables}

2.1. Two-photon decay branching ratio. . . . . . . . . . . . . . 14

3.1. Information on the two-photon experiment. . . . . . . . . . . . . . 24

4.1. Experimental results for the two-photon decay branching ratio. . . . . . . . . 33

5.1. Parameters of the QPM calculation of ${ }^{92,94} \mathrm{Zr}$ and ${ }^{94} \mathrm{Mo} \ldots \ldots \ldots \ldots \ldots$

6.1. Data taking conditions of the proton scattering experiments. . . . . . . . . . 62

7.1. Results of the ${ }^{92} \operatorname{Zr}\left(p, p^{\prime}\right)$ experiment. . . . . . . . . . . . . . . . . . 77

7.2. Results of the ${ }^{94} \mathrm{Zr}\left(p, p^{\prime}\right)$ experiment. . . . . . . . . . . . . . . . . . . . . . . . . .

7.3. Results of the ${ }^{94} \mathrm{Mo}\left(p, p^{\prime}\right)$ experiment. . . . . . . . . . . . . . . 83

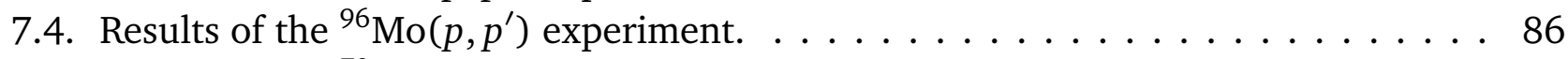

7.5. Results of the ${ }^{70} \mathrm{Zn}\left(p, p^{\prime}\right)$ experiment. . . . . . . . . . . . . . 90

8.1. Structure of the quadrupole QRPA-phonons in terms of two-quasiparticle states. . 97

8.2. Structure of the QPM wave function of the $2^{+}$states in terms of QRPA-phonons. . 99

8.3. Comparison of the calculated and measured transition strengths of $2^{+}$states. . . 100

8.4. Comparison of the calculated and measured g-factors of $2^{+}$states. . . . . . . 101

8.5. Structure of the hexadecapole QRPA-phonons in terms of two-quasiparticle states. 104

8.6. Structure of the hexadecapole QPM wave functions in terms of QRPA-phonons. . . 105

8.7. Comparison of the calculated and measured transition strengths of hexadecapole

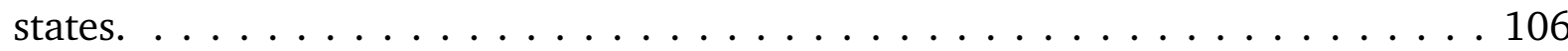

8.8. Comparison of the calculated and measured $g$-factors of hexadecapole states. . . 108

8.9. Structure of the octupole QRPA-phonons in terms of two-quasiparticle states. . . . 109

8.10. Structure of octupole and $5^{-}$QPM wave functions in terms of QRPA-phonons. . . 109

8.11. Comparison of the calculated and measured transition strengths of octupole and

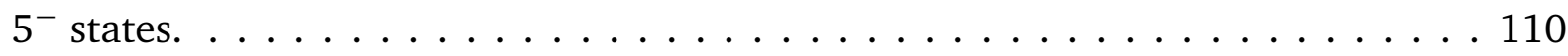

8.12. QRPA results for the $R$-value using the full QPM model space and only the main

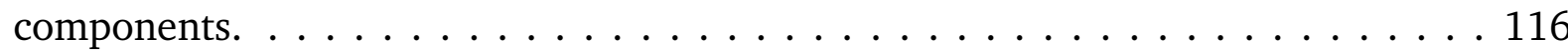

8.13. Proton, neutron and matter transition radii of the $2_{1}^{+}$and $2_{\mathrm{ms}}^{+}$states. $\ldots \ldots 123$

B.1. Cross sections of the ${ }^{94} \operatorname{Zr}\left(\mathrm{p}, \mathrm{p}^{\prime}\right)$ experiment. . . . . . . . . . . . . . . 135

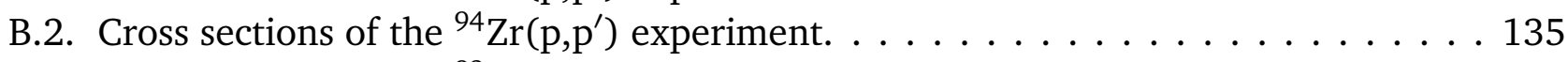

B.3. Cross sections of the ${ }^{92} \operatorname{Zr}\left(\mathrm{p}, \mathrm{p}^{\prime}\right)$ experiment. . . . . . . . . . . . . . . . . . . . . . . . . . .

B.4. Cross sections of the ${ }^{92} \operatorname{Zr}\left(\mathrm{p}, \mathrm{p}^{\prime}\right)$ experiment. . . . . . . . . . . . . . 136

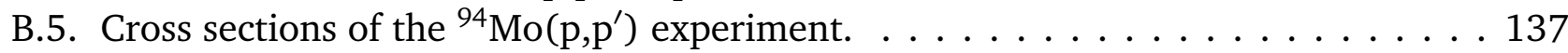

B.6. Cross sections of the ${ }^{94} \mathrm{Mo}\left(\mathrm{p}, \mathrm{p}^{\prime}\right)$ experiment. . . . . . . . . . . . . . . . . 137

B.7. Cross sections of the ${ }^{96} \mathrm{Mo}\left(\mathrm{p}, \mathrm{p}^{\prime}\right)$ experiment. . . . . . . . . . . . . . 138

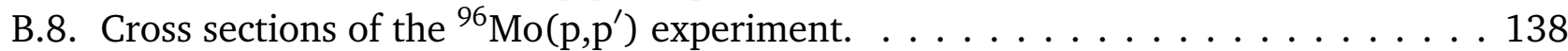

B.9. Cross sections of the ${ }^{70} \mathrm{Zn}\left(\mathrm{p}, \mathrm{p}^{\prime}\right)$ experiment. . . . . . . . . . . . . . . . 139 
B.10.Cross sections of the ${ }^{70} \mathrm{Zn}\left(\mathrm{p}, \mathrm{p}^{\prime}\right)$ experiment. . . . . . . . . . . . . . . 139 


\section{Danksagung}

An dieser Stelle ist es mir eine große Freude, allen denjenigen zu danken, die zum Gelingen dieser Arbeit beigetragen haben.

- Mein Dank gilt Prof. Dr. Norbert Pietralla für die Möglichkeit diese Arbeit durchzuführen. Seine Motivationsfähigkeit, Diskussionsbereitschaft und Offenheit gegenüber neuen Themen, die ursprünglich nicht in der Themenstellung der Doktorarbeit enthalten waren - wie dem Zwei-Photonenzerfall - haben entscheidend zum Gelingen dieser Arbeit beigetragen.

- Meine Arbeit über den Zwei-Photonenzerfall und über den Aufbau des $\mathrm{LaBr}_{3}$-Balls wäre nicht möglich gewesen ohne die tatkräftige Unterstützung, das technische Fachwissen und den Ideenreichtum von Dr. Heiko Scheit. Zudem danke ich ihm für die freundliche Übernahme des Zweitgutachtens.

- I am indebted to Dr. V. Yu. Ponomarev for the QPM calculations and numerous, fruitful discussions on the interpretation of the results of the proton scattering experiments.

- I want to thank Dr. Retief Neveling, Dr. Ricky Smith and all other members of the nuclear physics group at iThemba LABS for their great support with the successful proton scattering experiment in South Africa. Furthermore Retief Neveling helped with the analysis of the proton scattering data.

- Many thanks go to Prof. Dr. Franco Camera, Dr. Oliver Wieland and Stefano Riboldi for many useful suggestions on the $\mathrm{LaBr}_{3}$-ball and for sharing their constructional drawings of the active voltage divider.

- I want to thank Dr. H. T. Johansson for his ucesb and ggland software, H. Schaffner for his mbs-readout files and Dr. N. Kurz for his support to get the mbs-DAQ running.

- I wish to thank Dr. John Millener for generously sharing his experimental and theoretical results on the two-photon decay in ${ }^{137} \mathrm{Ba}$. His advices and suggestions helped me significantly to conduct my two-photon decay experiment successfully.

- Mein Dank gebührt allen aktuellen und ehemaligen Mitgliedern der Tagger-Gruppe, insbesondere Dr. Linda Schnorrenberger, Ronan Lefol, Bastian Löher, Dr. Dmytro Symochko und Diego Semmler.

- Insbesondere möchte ich meinen Bürokollegen Christian Stahl, Michael Reese und Christoph Krämer für eine angenehme Arbeitsatmosphäre und für die Unterstützung während des Protonenstreuexperiments in Südafrika danken. 
- Ich möchte allen Mitarbeitern der Mechanik- und der Elektronik-Werkstätten, des Strahlenschutzes und den Sekretariaten danken. Sie stellen durch ihren Einsatz überhaupt erst sicher, dass wissenschaftliches Arbeiten am Instiut für Kernphysik möglich ist. Insbesondere seien an dieser Stelle Herr Bonnes für seine Arbeit an dem Spanunngsteiler der $\mathrm{LaBr}_{3}$-Detektoren und Herr Flath für den Bau der Halterung des $\mathrm{LaBr}_{3}$-Balls erwähnt.

- Ein herzliches Dankeschön geht an meine Eltern - Inge und Dr. Gerd Walz - und an meine Geschwister - Susanne Walz und Dr. Michael Walz - für Rat und Unterstützung während meiner gesamten Ausbildung.

Die vorliegende Arbeit wurde gefördert durch Mittel der deutschen Forschungsgemeinschaft im Rahmen des Sonderforschungsbereichs 634. 


\section{Lebenslauf}

Der Lebenslauf ist in der Online-Verison aus Gründen des Datenschutzes nicht enthalten. 


\section{Erklärung zur Dissertation}

Hiermit versichere ich, die vorliegende Dissertation ohne Hilfe Dritter nur mit den angegebenen Quellen und Hilfsmitteln angefertigt zu haben. Alle Stellen, die aus Quellen entnommen wurden, sind als solche kenntlich gemacht. Diese Arbeit hat in gleicher oder ähnlicher Form noch keiner Prüfungsbehörde vorgelegen.

Darmstadt, den 13.6.2014

(Christopher Walz) 